\title{
Approximation des espaces de distributions et des opérateurs différentiels
}

Mémoires de la S. M. F., tome 12 (1967)

<http://www.numdam.org/item?id=MSMF_1967_12_3_0>

C Cémoires de la S. M. F., 1967, tous droits réservés.

L'accès aux archives de la revue « Mémoires de la S. M. F. » (http://smf. emath.fr/Publications/Memoires/Presentation.html) implique l'accord avec les conditions générales d'utilisation (http://www.numdam.org/conditions). Toute utilisation commerciale ou impression systématique est constitutive d'une infraction pénale. Toute copie ou impression de ce fichier doit contenir la présente mention de copyright.

\section{Numdam}


Bull. Soc. math. France,

Mémoire $12,1967,139 \mathrm{p}$.

APPROXIMATION DES ESPACES DE DISTRIBUTIONS ET DES OPERATEURS DIFFERENTIELS par Jean-Pierre AUBIN (*)

Que Monsieur J.L. LIONS veuille bien accepter ici l'expression de ma profonde reconnaissance pour n'avoir cessé de me prodiguer ses conseils et ses encouragements.

Je remercie Monsieur R. GODEMENT d'avoir bien voulu présider le jury de cette thèse et Monsieur J.P. KAHANE de me faire l'honneur d'y participer.

Ma reconnaissance va également à Monsieur M. GAUSSENS et à l'Electricité de France pour les facilités qui m'ont été accordées. Enfin, je remercie Monsleur J. CEA (dont les travaux ont été au point de départ de celui-c1) et Monsieur P.A. RAVIART pour leur amicale collaboration.

(*) Thèse Sc. math., Paris 1966 . 
CHAPITRE I - APPROXIMATION DES ESPACES DE BANACH ET DES OPERATEURS.

\$1 - APPROXIMATIONS D'UN ESPACE DE BANACH. 10

$\begin{array}{lll}1-1 & L \\ & & 10\end{array}$

1-2 Approximations d'un espace de Banach. 10

1-3 Stabilité - Convergence discrète et convergence. 11

1-4 Approximations partielles. $\quad 14$

1-5 Approximations duales. 14

1-6 Approximations autoadjointes. 16

1-7 Approximations discrètes. 16

1-8 Approximations de Galerkine. 18

1-9 Troncatures de Tchebychev. 20

1-10 Construction d'approximations. 21

$\$ 2$ - APPROXIMATION DES OPERATEURS. 23

2-1 Approximations des espaces d'opérateurs. 23

2-2 Les espaces $\pi E_{h}$ et $B E_{h}$ associés à $A_{h}(E)$. 24

2-3 Stabilité des opérateurs. 25

2-4 Approximation discrète des opérateurs. 27

2-5 Approximation falble des opérateurs. 30

2-6 Approximation des opérateurs compacts. 31

2-7 Approximation des opérateurs différentiables. 32

\$3 - APPROXIMATION DES OPERATEURS D'UN ESPACE DANS SON DUAL. 36

3-1 Formulation variationnelle. 37

3-2 Conditions suffisantes de stabilité des opérateurs $\mathrm{A}_{\mathrm{h}}^{-1} \cdot \quad 38$

3-3 Approximation par les restrictions d'un opérateur.

Etude de 1'erreur. $\quad 44$

3-4 Approximation par les restrictions partielles d'un $\begin{array}{ll}\text { opérateur A . } & 48\end{array}$

3-5 Approximation faible des opérateurs monotones. 51

3-6 Approximation des solutions définies par le lemme des projections. 
\$4 APPROXIMATION DES SOLUTIONS DES INEQUATIONS VARIATIONNELLES.

4-1 Position du problème. $\quad 57$

4-2 Théorèmes de convergence. 58

\$5 - APPROXIMATION DES SOLUTIONS DES EQUATIONS DIFFERENTIELLES 64 OPERATIONNELLES (I).

5-1 Rappels sur les équations différentielles-opérationnelles du le ordre. $\quad 64$

5-2 Approximation par des systèmes d'équations différentielles. 66

CHAPITRE II - APPROXIMATION DES ESPACES DE SOBOLEV ET DES SOLUTIONS D'EQUATIONS AUX DERIVEES PARTIELLES.

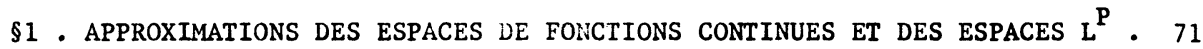

1-1 Notations. 71

1-2 Approximations discrètes stables et convergentes des espaces de fonctions continues. 73

1-3 Approximations des espaces $L^{P}(d \mu)$. 77

$\$ 2$ - APPROXIMATIONS DES ESPACES DE SOBOLEV SUR $\mathrm{R}^{\mathrm{n}}$. 80

2-1 Puissance de convolution des fonctions caractéristiques. 81

2-2 Approximations des espaces de Sobolev sur $\mathbf{R}^{\mathrm{n}}$. $\quad 85$

2-3 Approximation des espaces de distributions. 92

\$3 - APPROXIMATIONS DES ESPACES DE SOBOLEV SUR UN OUVERT $\Omega \cdot{ }^{\circ}$

3-1 Les réseaux $R_{h}^{K}(\Omega)$. 93

3-2 Approximations des espaces $W_{0}^{m, P}(\Omega)$. 95

3-3 Approximations des espaces $\mathrm{W}^{\mathrm{m}, \mathrm{P}}(\Omega)$. 99

3-4 Approximation de sous-ensembles convexes des espaces 104

\$4 . APPROXIMATION DES SOLUTIONS DE PROBLEMES AUX LIMITES POUR DES $\begin{array}{ll}\text { OPERATEURS DIFFERENTIELS LINEAIRES OU NON. } & 107\end{array}$

4-1 Rappels sur les problèmes elliptiques d'ordre $2 \mathrm{~m}$. 107

4-2 Approximation des solutions du problème de Neumann pour des opérateurs elliptiques d'ordre $2 \mathrm{~m}$. 110

4-3 Approximation des solutions des problèmes aux limites pour des opérateurs différentiels non linéaires. 
4-4 Approximation des solutions de problèmes aux limites unilatéraux - Calcul d'une capacité.

$\$ 5$ - APPROXIMATION DES SOLUTIONS DES EQUATIONS DIFFERENTIELLES

OPERATIONNELLES (II)

5-1 Convergence forte des solutions du schéma explicite. 120

5-2 Approximation des solutions des équations paraboliques. 127

5-3 Construction de schémas à plusieurs niveaux -

Majoration de l'erreur.

APPENCIDE 
Relation d'ordre dans la lecture des paragraphes :

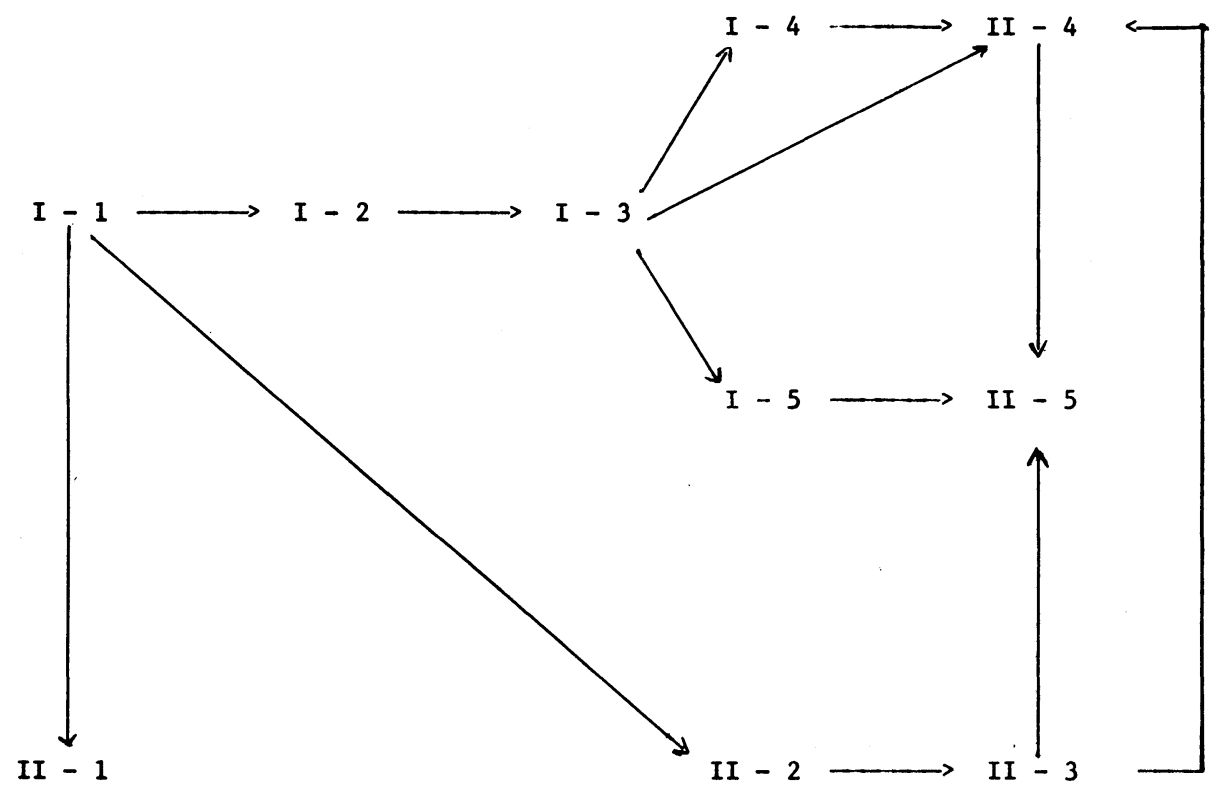


Notre but est d'approcher les solutions de divers problèmes différentiels, linéaires ou non, par des solutions de problèmes approchés aux "différences finies" et de donner une méthode générale de construction de ces problèmes approchés (Chap II, $\$ 4$ et $\$ 5)$.

Sous son aspect général, le problème se présente de la façon suivante:

Soit A un opérateur (1inéaire ou non) d'un espace de Banach $E$ dans un autre espace de Banach $F$; on admet qu'il existe une solution du

problème $P$. Chercher $u$ dans $E$ tel que $A(u)=f$ donné dans $F$.

On se donne un paramètre $h$ destiné à tendre vers 0 , auquel on associe un opérateur $A_{h}$ d'un espace $E_{h}$ dans un espace $F_{h}$ (ces espaces étant de dimension finie) et on remplace le problème $P$ par le

problème $P_{h}$. Chercher $u_{h}$ dans $E_{h}$ tel que $A_{h}\left(u_{h}\right)=f_{h}$ dans $F_{h}$.

On se pose alors les questions suivantes :

- En quel sens définir la "convergence" de $u_{h}$ vers $u$ ?

- Quelles conditions doivent satisfaire les opérateurs $A_{h}$ pour que la "convergence" des données entraîne celle des solutions?

- Comment construire des opérateurs $A_{h}$ vérifiant ces conditions ?

Généralement, pour définir la convergence, on munit chacun des $E_{h}$ d'une norme $\left\|u_{h}\right\|_{h}$, on se donne un opérateur $r_{h}$ de $E$ dans $E_{h}$ (celui, par exemple, qui à toute fonction continue associe ses valeurs aux points d'un réseau) et on dit que $u_{h}$ converge discrètement vers $u$ si $\left\|u_{h}-r_{h} u\right\|_{h}$ tend vers 0 avec $h$. Cette notion dépend du choix des normes $\left\|u_{h}\right\|_{h}$ sur $E_{h}$, même si, pour $h$ fixé, ces normes sont équivalentes. Nous introduirons alors une autre notion ; nous construirons des opérateurs linéaires continus de $E_{h}$ dans $E$ et nous dirons que $u_{h}$ converge vers $u$ (fortement ou faiblement) si $p_{h} u_{h}$ converge vers $u$ dans $E$ (fort ou faible). La convergence discrète entraîne la convergence forte si (et seulement si) ||$p_{h} u_{h}\left\|_{E} \leqslant M|| u_{h}\right\|_{h} \quad\left(M\right.$ indépendante de $h$ ) et $p_{h} r_{h} u$ converge vers $u$ pour tout $u$. (Chap $I ; \$ 1$, théorème 1 du $\$ 1$ ). Ainsi nous définirons une approximation $A_{h}(E)$ de $E$ dans $E_{h}$ :

$$
A_{h}(E)=\left(E, E_{h}, P_{h}, r_{h}\right)
$$

par la donnée d'un opérateur $p_{h}$ de $E_{h}$ dans $E$ et d'une application $r_{h}$ de $E$ dans $E_{h} \cdot($ Chap I, $\$ 1)$.

Donnons-nous maintenant une approximation $A_{h}(F)=\left(F, F_{h}, q_{h}, s_{h}\right)$ de $F$ 
dans $F_{h}$. Les hypothèses à faire sur les opérateurs $A_{h}$ pour que la convergence discrète de $f_{h}$ vers $f$ entraîne celle de $u_{h}$ vers $u$ sont les suivantes (Chap I, $\$ 2$, théorème 1 du $\$ 2$ ) :

- Les $A_{h}$ sont consistants à $A$ en $u$, c'est-à-dire :

$$
\| A_{h}\left(r_{h} u\right)-\left.s_{h} A(u)\right|_{F_{h}} \text { tend vers } 0 \text { avec } h
$$

- Les opérateurs $A_{h}^{-1}$ sont stabies, c'est-à-dire : les opérateurs $A_{h}^{-1}$ sont globalement (en $\mathrm{h}$ ) uniformément continus :

Pour tout $\varepsilon$, il existe $n$ tel que $\left\|f_{h}-g_{h}\right\|_{h} \leqslant n$ entraine :

$$
\left\|A_{h}^{-1}\left(f_{h}\right)-A_{h}^{-1}\left(g_{h}\right)\right\|_{h} \leqslant \varepsilon \quad \text { pour tout } h \text {. }
$$

Si les opérateurs $A_{h}$ sont linéaires, on retrouve la notion classique de stabilité :

$$
\left\|A_{h}^{-1} f_{h}\right\|_{h} \leqslant M\left\|f_{h}\right\|_{h} ; M \text { indépendante de } h \text {. }
$$

Enfin, comment construire les opérateurs $A_{h}$ consistants à $A$, inversibles, et tels que les $A_{h}^{-1}$ soient stables ? on considère le schéma suivant :

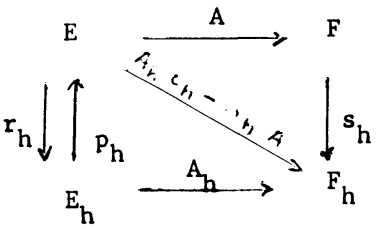

Considérons alors l'opérateur particulier suivant :

$$
A_{h}=s_{h} A p_{h}
$$

Nous étudierons alors les conditions à imposer aux opérateurs $\mathrm{p}_{h}$ et $s_{h}$ pour que, dans une classe d'opérateurs donnée, les conditions précédentes soient satisfaites (Chap I , $\$ 2, \$ 3, \$ 4$ et 5 ) et nous étudierons spécialement le comportement de l'erreur.

Nous considérerons en particulier la classe des opérateurs différentiables (au sens de Fréchet), des opérateurs d'un espace sur son dual, des opérateurs définis par des inéquations variationnelles, des équations différentielles opérationnelles.

Ainsi ramenons-nous au chapitre I, l'étude de l'approximation des solutions du problème $P^{\prime}$ à la construction d'approximations des espaces $E$ et $F$ vérifiant certaines propriétés. Ces approximations sont indépendantes du choix d'un opérateur

A particulier et permettent de construire des problèmes approchés en explicitant les opérateurs $A_{h}=r_{h} A p_{h}$. 
Les premiers paragraphes du chapitre II sont donc consacrés à la construction d'approximations des espaces de distributions. Puisque maints problèmes différentiels sont posés dans les espaces de Sobolev, nous étudions plus spécialement leurs approximations (Chap II, $\$ 2$ et $\$ 3$ ). Les $\$ 4$ et $\$ 5$ développent quelques exemples d'approximation des solutions des problèmes aux limites (même unilatéraux) pour des opérateurs différentiels linéaires ou non, des solutions d'équations paraboliques. Nous obtenons alors des problèmes approchés du type "schémas aux différences finies", parmi lesquels nous retrouvons les schémas classiques.

CHAPITRE 1.

APPROXIMATION DES ESPACES DE BANACH ET DES OPERATEURS

§1. APPROXIMATIONS D'UN ESPACE DE BANACH.

Nous donnons aux $\mathrm{n}^{\circ} 1.1$ à 1.6 les définitions et les propriétés des approximations que nous utiliserons par la suite.

Les numéros 1.7 à 1.10 donnent des premiers exemples d'approximations.

Signalons que $l^{\prime}$ on peut définir des approximations d'espaces plus généraux que des espaces de Banach : En particulier, on peut définir des approximations d'un espace topologique complètement régulier (c'est-à-dire uniformisable et séparé). Mais cette généralisation ne nous est pas utile ici.

On peut aborder dès la fin de ce paragraphe, les $\$ 1$, $\$ 2$ et $\$ 3$ du chapitre II, où $l^{\prime}$ on trouvera des exemples d'approximations des espaces de distributions.

\section{1-1. L'ENSEMBLE Z}

Nous désignerons par $Z$ un ensemble de paramètres $h$ muni d'un filtre $F$ à base dénombrable. Pour simplifter les notations, nous supposerons que $Z$ est un sous-ensemble d'un espace vectoriel de dimension finie, dont 1 'adhérence contient 1 'origine. Nous prendrons pour filtre $F$ la trace sur $z$ du filtre des voisinages de 1 'origine.

\section{1-2. APPROXIMATIONS D'UN ESPACE DE BANACH}

DEFINITION $1=1$.

Nous appelerons approximation (d'ordre $h / A_{h}(E)$ d'un espace de Banach $E$ dans un espace de Banach $\mathrm{E}_{\mathrm{h}}$ la donnée : 


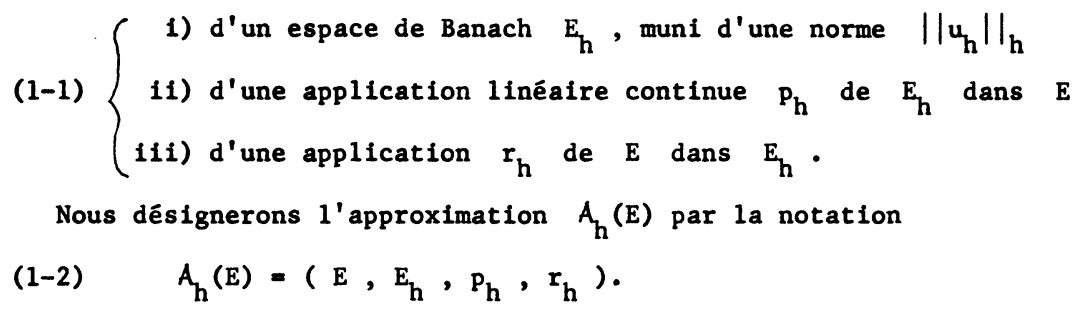

et nous dirons que :

$(1-3)\left\{\begin{array}{rll}\text { i) } & P_{h} \text { est le prolongement de 1'approximation } A_{h}(E) \\ \text { ii) } & r_{h} \text { est la restriction de 1'approximation } A_{h}(E) \\ \text { iii) } & P_{h} r_{h} & \text { est la troncature de 1'approximation } A_{h}(E)\end{array}\right.$

Si u appartient à $E$ et $u_{h}$ appartient à $E_{h}$, nous dirons que :

(1-4) $\left\{\begin{aligned} \text { i) }|| u_{h}-\left.r_{h} u\right|_{h} \text { est l'erreur discrète entre } u \text { et } u_{h} \\ \text { ii) } u-p_{h} u_{h} \text { est l'erreur entre u et } u_{h} . \\ \text { iii) u- } p_{h} r_{h} u \text { est l'erreur de troncature de } u \text {. }\end{aligned}\right.$

REMARQUE $1-1$.

La donnée de la norme $\left\|u_{h}\right\|_{h}$ de l'espace $E_{h}$ entre dans la définition de $A_{h}(E)$ : Si l'on remplace la norme $\left\|u_{h}\right\|_{h}$ par une norme équivalente \|\|$_{h}\|\|_{h}$, nous obtenons une approximation différente : en effet, 11 existe bien des constantes $c(h)$ et $c(h)$ telles que

$$
\left\|u_{h}\right\|_{h} \leqslant c(h)\left|\left\|u_{h}\right\|\left\|_{h} \leqslant c(h)|| u_{h}\right\|_{h}\right.
$$

mais ces constantes peuvent tendre vers 1 'infini quand $h$ tend vers 0 . (cf. $\$ 2$, $n^{\circ} 2-2$ ).

EXEMPLE 1-1

Si $E_{h}$ est un espace de dimension finie et $s i p_{h}$ est injectif, alors (1-5) $\quad\left\|u_{h}\right\|_{h}=\left\|p_{h} u_{h}\right\|$

est une norme. Ce choix de la norme $\left\|u_{h}\right\|_{h}$ ne convient pas toujours (cf. $n^{\bullet} 1-4$ et 1-5).

1-3. STABILITE, CONVERGENCE DISCRETE ET CONVERGENCE.

Considérons à présent une famille $A_{h}(E)$ d'approximations de $E$ lorsque $h$ parcourt $\mathrm{Z}$. Nous sommes conduits à faire des hypothèses globales sur ces approximations. 
DEFINITION 1-2.

Nous dirons que les prolongements sont stables s'il existe une constante $M$ indépendante de $h$ telle que

$$
\left\|p_{h} u_{h}\right\| \leqslant M \mid u_{h} \|_{h} \quad \text { pour tout } h \text { et pour tout } u_{h} \text { de } E_{h} \text {. }
$$

Nous dirons que les restrictions $r_{h}$ sont stables si

(1-7) $\left\{\begin{array}{l}\text { Pour tout } \varepsilon, \text { il existe } n \text { tel que } \\ \|u-v\| \leqslant n \text { entraine }\left\|r_{h} u-r_{h} v\right\|_{h} \leqslant \varepsilon \quad \text { pour tout } h\end{array}\right.$

Nous dirons qu'une famille $\pi u_{h}=\left(u_{h}\right)_{h}$ d'éléments $u_{h}$ de $E_{h}$ :

a) converge discrètement vers un élément u de E si

$$
\lim _{h=0}|| u_{h}-r_{h} u \|_{h}=0
$$

b) converge fortement (resp. faiblement) vers u si

(1-9) $\quad P_{h} u_{h}$ converge fortement (resp. faiblement) vers $u$ dans $E$.

Nous dirons enfin que les approximations sont stables si les prolongements et les restrictions sont stables et que les approximations sont convergentes si les erreurs de troncature tendent vers 0 :

(1-10) $\quad \lim _{\mathrm{h}=0}|| \mathrm{u}-\mathrm{p}_{\mathrm{h}} \mathrm{r}_{\mathrm{h}} \mathrm{u} \|=0$ pour tout $\mathrm{u}$ dans $\mathrm{E}$.

Nous dirons aussi que les restrictions $r_{h}$ sont globalement bonnées si

(1-11) $\quad\left\|r_{h} u\right\|_{h} \leqslant m(u)\|u\| \quad$ pour tout $h$.

Si les restrictions $r_{h}$ sont linéaires, les conditions (1-7) et (1-11) sont équivalentes.

Dire que les approximations $A_{h}(E)$ sont convergentes est dire que tout élément $u$ de $E$ est limite d'une famille $p_{h} u_{h}$, ou encore que $\bigcup_{h} p_{h} E_{h}$ est dense dans
$E$.

Inversement, si $\bigcup_{h} p_{h} E_{h}$ est dense dans $E$ et si $p_{h} E_{h} C p_{k} E_{k}$ pour $k<h$, il existe des restrictions $r_{h}$ telles que les $A_{h}(E)$ solent convergentes.

Remarquons enfin que la notion de convergence discrète dépend du choix des normes $\left\|u_{h}\right\|_{h}$ de $E_{h}$.

L'introduction de ces définitions est motivée par le :

THEOREME $1-1$.

Pour que la convergence discrète implique la convergence forte, il faut et il suffit que : 
a) les prolongements soient stables

b) Les approximations $A_{h}(E)$ soient convergentes.

L'inégalité

$$
\left\|u-p_{h} u_{h}\right\| \leqslant\left\|u-p_{h} r_{h} u\right\|+\left\|p_{h}\left(u_{h}-r_{h} u\right)\right\|
$$

montre que les conditions a) et b) sont suffisantes. La condition b) est nécessaire puisque $r_{h} u$ converge discrètement vers u par définition. On suppose que la condition a) n'est pas nécessaire : il existe alors une famille $\left(u_{h}\right)_{h}$ telle que :

$$
\left\|u_{h}\right\|_{h} \leqslant 1 \text { et } n_{h}=\left\|p_{h} u_{h}\right\| \text { tend vers l'infini. }
$$

Si $v_{h}=\eta_{h}^{-1 / 2} u_{h}$, on en déduit que $v_{h}$ converge discrètement vers 0 et que $p_{h} v_{h}$ tend vers l'infini, ce qui contredit l'hypothèse.

Si l'on suppose de plus que les restrictions sont linéaires et stables, on déduit de l'inégalité :

$$
|| p_{h} r_{h} u|| \leqslant M|| r_{h} u||_{h} \leqslant m M|| u||
$$

que

(1-12) $\quad\|\mid u\|\left\|=\lim _{h=0}\right\| r_{h} u \|_{h}$ est une norme équivalente à $\|u\|$.

PROPOSITION 1-1.

Supposons que les approximations soient stables. Pour que les approximations soient convergentes, il faut et il subjit que:

$$
\lim _{h=0}\left\|p_{h} r_{h} u-u\right\|=0 \text { pour tout } u \in P ; P \text { dense dans } E \text {. }
$$

Dans ce cas $\mathrm{p}_{h} \mathrm{r}_{\mathrm{h}} \mathrm{u}$ converge vers $\mathrm{u}$ uniformément sur tout compact de $\mathrm{E}$.

En effet, si les prolongements et les restrictions sont stables, les troncatures $p_{h} r_{h}$ forment un ensemble équicontinu d'applications de $E$ dans lui-même, d'où la proposition.

COROLLAIRE 1-1.

Supposons que l'injection de $\mathrm{K}$ dans $\mathrm{E}$ soit compacte, et que les approximations $A_{h}(E)$ soient stables et convergentes.

Alors, si les restrictions sont linéaires:

$$
r(h)=\sup _{u \in K} \frac{\left\|u-p_{h} r_{h} u\right\|}{\|u\|_{K}} \text { tend vers } 0 \text { avec } h \text {. }
$$


Cela revient à montrer que les troncatures $p_{h} r_{h}$ convergent uniformément sur tout borné de $K$, qui est relativement compact dans $E$. Mais cela résulte de la proposition précédente.

\section{1-4. APPROXIMATIONS PARTIELLES}

Supposons que $I^{\prime}$ espace $E$ soit un sous espace fermé de 1 'intersection d'une suite finie d'espaces $E_{q}$ (que nous supposerons tous plongés dans un même espace localement convexe séparê..)

$$
E \subset \bigcap_{q} E_{q} \quad ; \quad\|u\|=\sup _{q}\|u\|_{q}
$$

DEFINITION 1-3.

Nous dirons que les approximations de $\mathrm{E}_{\mathrm{q}}$ dans $\mathrm{E}_{\mathrm{h}}$ :

(1-14) $A_{h}\left(E_{q}\right)=\left(E_{q}, E_{h}, p_{h}^{q}, r_{h}\right)$

soni des approximations partielles de $\mathrm{E}$ si :

(1-15) $\left\{\begin{array}{l}\mathrm{p}_{\mathrm{h}}^{\mathrm{q}} \mathrm{u}_{\mathrm{h}} \text { tend vers } \mathrm{u}_{\mathrm{q}} \text { dans } \mathrm{E}_{\mathrm{q}} \text { faible pour tout } \mathrm{q} \text {, tous lés éléments } \\ \mathrm{u}_{\mathrm{q}} \text { sont égaux à un même élément } \mathrm{u} \text { de } \mathrm{E}\end{array}\right.$

Nous dirons que les approximations $A_{h}(E)=\left(E, E_{h}, p_{h}, r_{h}\right)$ sont plus fines que les approximations partielles $A_{h}\left(E_{q}\right)$ si

(1-16) $\left\{\begin{array}{l}\text { 1) }\left\|p_{h} u_{h}\right\| \leqslant \sup _{q}\left\|p_{h}^{q} u_{h}\right\|_{q} \\ \text { ii) }\left\|p_{h}^{q} u_{h}\right\|_{q} \leqslant k \text { entraine } \lim _{h=0}\left(p_{h} u_{h}-p_{h}^{q} u_{h}\right)=0 \text { dans } E_{q} \text { faible. }\end{array}\right.$

Nous supposerons que $1^{\prime}$ une des expressions suivantes :

$$
\left\{\begin{array}{l}
\text { i) }\left\|u_{h}\right\|_{h}=\sup _{q}\left\|p_{h}^{q} u_{h}\right\|_{h} \\
\text { ii) }\left\|u_{h}\right\|_{h}=\left(\sum_{q}\left\|p_{h}^{q} u_{h}\right\|^{\alpha}\right)^{1 /}{ }_{\alpha} ; 1 \leqslant \alpha \leqslant+\infty
\end{array}\right.
$$

est une norme de $E_{h}$.

Si les approximations $A_{h}\left(E_{q}\right)$ sont convergentes, dire alors que $u_{h}$ converge discrètement vers $u$ est dire que

(1-18) $\lim _{h=0} \sup _{q}\left\|u-p_{h}^{q} u_{h}\right\|_{q}=0$

\section{1-5. APPROXIMATIONS DUALES}

Solent $E$ (resp. $E_{h}$ ) un espace de Banach, $E^{\prime}$ (resp. $\left.E_{h}^{\prime}\right)$ son dual, (f, u)

(resp. $\left.\left(f_{h}, u_{h}\right)_{h}\right)$ une forme bilinéaire mettant $E$ et $E^{\prime}$ (resp. $E_{h}$ et $E_{h}^{\prime}$ ) en 
dualité.

Considérons les normes duales sur $E^{\prime}$ et $E_{h}^{\prime}$ :

(1-19) ||$f\left\|_{*}=\sup _{v}|| v||^{-1}|(f, v)| ;|| f||_{\star h}=\sup _{v_{h}}|| v_{h}\left|\|_{h}^{-1}\right|\left(f_{h}, v_{h}\right)_{h} \mid\right.$

Considérons des approximations $A_{h}(E)=\left(E, E_{h}, p_{h}, r_{h}\right)$ où les restrictions sont linéaires.

DEFINITION $1-4$.

Nous dirons que l'approximation du dual E' de E :

$$
A_{h}{ }^{*}\left(E^{\prime}\right)=\left(E^{\prime}, E_{h}, P_{h}{ }^{*}, r_{h}{ }^{*}\right)
$$

où $E_{h}$ est muni de la norme $\left\|f_{h}\right\|_{* h}$, où $p_{h}{ }^{*}$ est la transposée de $\mathbf{r}_{h}$ et $\mathbf{r}_{\mathrm{h}}{ }^{*}$ la transposée de $\mathrm{P}_{\mathrm{h}}$ :

(1-20) $\left(p_{h}{ }^{*} f_{h}, v\right)=\left(f_{h}, r_{h} v\right)_{h} ;\left(r_{h}{ }^{*} f, v_{h}\right)_{h}=\left(f, p_{h} v_{h}\right)$

est l'approximation duale $A_{h}{ }^{*}\left(E^{\prime}\right)$ de $A_{h}(E)$

On déduit des propriétés des applications transposées 1a :

PROPOSITION 1-2.

Si les prolongements $p_{h}$ (resp. les restrictions $r_{h}$ ' sont stables, les restrictions $r_{h}{ }^{*}$ (resp. les prolongements ${p_{h}}^{*}$ ) sont également stables. Si les approximations $A_{h}(E)$ sont convergentes, les troncatures $P_{h}{ }^{*}{ }_{h}{ }^{*}$ convergent faiblement et uniformément sur tout borné de E' vers. l'identité.

En effet, la norme de $p_{h}{ }^{*}$ est égale à celle de $r_{h}$ et la norme de $r_{h}{ }^{*} a ̀$ celle de $p_{h}$. De plus, si $B$ est un borné de $E^{\prime}$ :

$\lim _{h=0} \sup _{f \in B}\left|\left(p_{h}{ }^{*} r_{h}{ }^{*} f-f, u\right)\right| \leqslant \lim _{h=0} \sup _{f \varepsilon B}|| f||_{\star}|| p_{h} r_{h} u-u||$

Dire que $f_{h}$ converge discrètement vers $f$ dans $E^{\prime}$ est dire que :

(1-21) $\left\|r_{h}{ }^{*} f-f_{h}\right\|_{h}=\sup _{v_{h}}\left\|v_{h}\right\|_{h}^{-1}\left|\left(f, p_{h} v_{h}\right)-\left(f_{h}, v_{h}\right)_{h}\right|=0$ 
1-6. APPROXIMATIONS AUTOADJOINTES.

Supposons que $E_{h}$ : est un espace de dimension finie, identifié à son dual $E_{h}^{\prime}$. Supposons de plus que

$$
p_{h} E_{h} \subset E \cap E^{\prime} \quad \text { pour tout } h \text {. }
$$

Puisque $E_{h}$ est de dimension finie :

$$
P_{h} \varepsilon L\left(E_{h}, E\right) \cap L\left(E_{h}^{\prime}, E^{\prime}\right) ; r_{h}^{*} \varepsilon L\left(E, E_{h}\right) \cap L\left(E^{\prime}, E_{h}^{\prime}\right)
$$

DEFINITION $1-5$.

Sous les conditions (1-22) et (1-23), nous dirons que les approximations

$A_{h}(E)=\left(E, E_{h}, P_{h}, r_{h}{ }^{*}\right)$ et $A_{h}\left(E^{\prime}\right)=\left(E^{\prime}, E_{h}^{\prime}, P_{h}, r_{h}{ }^{*}\right)$ sont des approximations autoadjointes de $\mathrm{E}$ et de $\mathrm{E}^{\prime}$.

Dire alors que les approximations autoadjointes $A_{h}(E)$ sont stables est dire que les prolongements $p_{h}$ sont stables dans $A_{h}(E)$ et dans $A_{h}\left(E^{\prime}\right)$ :

$$
\left\|p_{h} u_{h}\right\| \leqslant M \mid u_{h}\left\|_{h} \quad ; \quad\right\| p_{h} f_{h}\left\|_{*} \leqslant m\right\| f_{h} \|_{* h}
$$

PROPOSITION 1-3.

Supposons que E s'identifie à un sous espace dense de $E^{\prime}$. Si les approximations autoadjointes $A_{h}(E)$ sont stables et convergentes, les approximations autoadjointes $A_{h}\left(E^{\prime}\right)$ sont également stables et convergentes.

On sait déjà que les approximations $A_{h}\left(E^{\prime}\right)$ sont stables et faiblement convergentes. D'après la proposition $1-1$, il suffit de montrer que $p_{h} r_{h}^{*} f$ converge vers $f$ dans $E^{\prime}$ pour $f$ de $E$. Mais cela résulte de ce que

$$
\left\|p_{h} r_{h}^{*} f-f\left|\|_{*} \leqslant\right|\left|p_{h} r_{h}^{*} f-f\right| \mid \text { tend vers } 0\right. \text {. }
$$

\section{1-7. APPROXIMATIONS DISCRETES.}

Nous allons donner un procédé de construction d'approximations de E . Associons à tout paramètre $h$ un ensemble $R_{h}$ (dénombrable) d'indices a et des suites $\sigma_{h}$ et $S_{h}$ dans $E$ et $E^{\prime}$ respectivement :

(1-24) $\begin{cases}\sigma_{h}=\left(\sigma_{h}{ }^{\alpha}\right)_{\alpha} \varepsilon R_{h} & ; \sigma_{h}^{\alpha} \varepsilon E \\ s_{h}=\left(s_{h}{ }^{\alpha}\right)_{\alpha} \varepsilon R_{h} & ; s_{h}^{\alpha} \varepsilon E^{\prime}\end{cases}$ 
DEFINITION 1-6.

Nous dirons que les suites $\sigma_{h}$ et $S_{h}$ définissent une approximation discrète de $E$ désignée par $A_{h}(E)=\left(E, \sigma_{h}, S_{h}\right)$ si les conditions suivantes sont satisfaites :

$$
\mathrm{p}_{\mathrm{h}} \mathrm{r}_{\mathrm{h}} \mathrm{u}=\sum_{\alpha \varepsilon R_{\mathrm{h}}}\left(\mathrm{S}_{\mathrm{h}}^{\alpha}, \mathrm{u}\right) \sigma_{\mathrm{h}}^{\alpha} \text { est continue de } \mathrm{E} \text { dans } \mathrm{E} \text {. }
$$

Une approximation discrète est une approximation

$$
A_{h}(E)=\left(E, E_{h}, p_{h}, r_{h}\right) \text { de } E \text { avec }
$$

$$
(1-26)\left\{\begin{array}{l}
\text { i) } E_{h}=1 \text { 'espace des suites } r_{h} u=\left(\left(s_{h}^{\alpha}, u\right)\right)_{\alpha \in R_{h}} \\
\text { ii) } p_{h} u_{h}=\sum_{\alpha \varepsilon R_{h}} u_{h}^{\alpha} \sigma_{h}^{\alpha} \\
\text { iii) } r_{h} u=\left(\left(s_{h}^{\alpha}, u\right)\right)_{\alpha \in R_{h}}
\end{array}\right.
$$

En effet, si $E_{h}$ est muni de la topologie la moins fine rendant continue $p_{h}$, la restriction $r_{h}$ est continue d'après $(1-25)$.

La condition (1-25) est toujours satisfaite si les suites $\sigma_{h}$ et $s_{h}$ sont finies.

On remarque que si la suite $S_{h}$, est topologiquement libre dans $E^{\prime}, 1$ 'espace $E_{h}$ contient les suites $u_{h}$ à support fini $\left(u_{h}{ }^{\alpha}=0\right.$ sauf pour un nombre fini d'indices $\alpha$ )., si $E$ est réflexif.

Si les suites $\sigma_{h}$ et $S_{h}$ sont finies et linéairement indépendantes, $E_{h}$ est un espace de dimension finie $N(h), p_{h}$ est un isomorphisme et $r_{h}$ un homomorphisme.

PROPOSITION 1-4.

Supposons que les suites $\sigma_{h}$ et $S_{h}$ soient biorthogonales :

$$
\left(s_{h}{ }^{\alpha}, \sigma_{h}{ }^{\beta}\right)=\left\{\begin{array}{lll}
0 & \text { si } & \alpha \neq \beta \\
1 & \text { si } & \alpha=\beta
\end{array}\right.
$$

et vérifiant (1-25).

Alors $\mathrm{p}_{\mathrm{h}}$ est un isomorphisme, $\mathrm{r}_{\mathrm{h}}$ est un homomorphisme et $\mathrm{p}_{\mathrm{h}} \mathrm{r}_{\mathrm{h}}$ est un projecteur continu de $E$ sur $\mathrm{p}_{\mathrm{h}} \mathrm{E}_{\mathrm{h}}$. 
L'hypothèse (1-27) entraíne que $r_{h} p_{h}$ est l'identité de $E_{h}$ :

$$
\left(r_{h} p_{h} u_{h}\right)^{\alpha}=\left(s_{h}^{\alpha}, \sum_{\beta} u_{h}^{\beta} \sigma_{h}^{\beta}\right)=u_{h}^{\alpha} \quad ; \alpha \in R_{h} \text {. }
$$

Il en résulte donc que $p_{h}$ est inversible à gauche et $r_{h}$ inversible à droite et ces applications sont continues. De plus, E est la somme directe topologique du noyau de $r_{h}$ et de $p_{h} E_{h}$.

Si une approximation discrète $A_{h}(E)=\left(E, \sigma_{h}, S_{h}\right)$ vérifie (1-27), nous dirons que $c^{\prime}$ est une interpolation, puisque $p_{h} u_{h}$ est une solution du problème :

Trouver $u$ dans $E$ vérifiant :

$$
\left(s_{h}^{\alpha}, u\right) u_{h}^{\alpha} \quad \text { pour tout } \alpha \text { de } R_{h} \text {. }
$$

On remarque enfin que l'approximation duale d'une approximation discrète est :

(1-28) $\quad A_{h}^{*}\left(E^{\prime}\right)=\left(E^{\prime}, S_{h}, \sigma_{h}\right)$.

Si $\sigma_{h}$ est une suite à valeurs dans $E \cap E^{\prime}$ telle que

(1-29) $\quad \mathrm{p}_{h} \mathrm{r}_{h}^{*} \mathrm{u}=\sum_{\alpha}\left(\sigma_{h}, u\right) \sigma_{h}^{\alpha}$ soit continue

l'approximation discrète $A_{h}(E)=\left(E, \sigma_{h}, \sigma_{h}\right)$ est autoadjointe.

On trouvera dans J. DIEUDONNE [1] l'étude des suites biorthogonales dans un espace vectoriel topologique. Au sujet des problèmes d'interpolation et d'approximation optimales, voir J.P. AUBIN [7] .

\section{1-8. APPROXIMATIONS DE GALERKINE.}

Soit $E$ un espace de Banach séparable. Il existe alors (au moins) une suite dénombrable d'éléments $\omega^{\alpha}$ de $E(\alpha=1,2, \ldots, n, \ldots)$, totale dans $E$, que 1 'on peut toujours supposer (algébriquement) 1ibre. On construit à partir de cette suite une approximation de $E$ de la manière suivante :

On prend :

$(1-30)\left\{\begin{array}{l}\text { i) } h=1 / n, n \in \mathbb{N} ; E_{h}=\mathbb{C}^{n} ; u_{h}=\left(u_{h}^{\alpha}\right)_{1 \leqslant \alpha \leqslant n} \varepsilon \mathbb{C}^{n} \\ \text { ii) } p_{h} u_{h}=\sum_{\alpha=1}^{n} u_{h}^{\alpha} \omega^{\alpha} ;\left\|u_{h}\right\|_{h}=\left\|p_{h} u_{h}\right\| \\ \text { iii) } r_{h} u \text { est telle que } p_{h} r_{h} u \text { converge vers } u \text { quand } n \text { tend vers } \infty .\end{array}\right.$

Nous dirons que de telles approximations sont des approximations de GALERKINE de E.

Si l'espace $E$ admet une base de Schauder $\omega^{\alpha}$ (cf. N. DUNFORD - J. SCHWARTZ $|1|$, p. 93 ), 11 existe une suite scalaire $u^{\alpha}$ telle que : 
$u=\sum_{\alpha=1}^{\infty} u^{\alpha} \omega^{\alpha}$

On peut prendre alors pour restriction (non linéaire) $: r_{h} u=\left(u^{1}, \ldots, u^{n}\right)$.

Si $H$ est un espace de Hilbert séparable et si $\omega^{\alpha}$ est une base orthonormale de H , nous obtenons des approximations de Galerkine autoadjointes, stables et convergentes, en prenant :

$$
r_{h} u=\left(\left(u, \omega^{\alpha}\right)\right)_{1 \leqslant \alpha \leqslant n}
$$

Plus généralement, soient $V$ et $H$ deux espaces de Hilbert tels que (1-31) l'injection de $\mathrm{V}$ dans $\mathrm{H}$ est compacte; $\mathrm{V}$ est dense dans $\mathrm{H}$.

Rappelons que (cf. Appendice)

$\mathrm{V} \subset \mathrm{H} \subset \mathrm{V}^{\prime}$, les injections étant denses et compactes.

L'opérateur non borné $\Lambda=\{\mathrm{V}, \mathrm{H},((\mathrm{u}, \mathrm{v}))\}$ est hermitien et son inverse $\mathrm{J}$ est compact. Les espaces de Hilbert $V, H, V^{\prime}$ sont munis des produits scalaires hermitiens suivants :

$\begin{cases}((\mathbf{u}, \mathbf{v}))=(\Lambda \mathbf{u}, \mathbf{v})=((\Lambda \mathbf{u}, \Lambda \mathbf{v}))_{*} & \text { sur } \mathbf{s} \\ (\mathbf{u}, \mathbf{v})=((\Lambda \mathbf{u}, \mathbf{v}))_{*}=((\mathrm{J}, \mathbf{v})) & \text { sur } \mathrm{H} \\ ((\mathbf{u}, \mathbf{v}))_{*}=(\mathrm{Ju}, \mathbf{v})=((\mathrm{J}, \mathrm{J} \mathbf{v})) & \text { sur } \mathbf{v}^{\prime}\end{cases}$

D'autre part, il existe une base orthonormale de $H$ formée des vecteurs propres de 1 'opérateur $\Lambda$ :

$$
\Lambda \omega^{\alpha}=\lambda_{\alpha} \omega^{\alpha} \quad ; \quad\left(\omega_{\alpha}, \omega_{\beta}\right)=\delta_{\alpha \beta} .
$$

Désignons alors par $V_{h}, H_{h}$ et $V_{h}^{\prime} I^{\prime}$ espace $\mathbb{C}^{n}$ muni des produits scalaires suivants :

$(1-33)\left\{\begin{array}{l}\left(\left(u_{h}, v_{h}\right)_{h}=\sum_{\alpha=1}^{n} \lambda_{\alpha} u_{h}^{\alpha} \bar{v}_{h}^{\alpha}=\left(\left(p_{h} u_{h}, p_{h} v_{h}\right)\right)\right. \\ \left(u_{h}, v_{h}\right)_{h}=\sum_{\alpha=1}^{n} u_{h}^{\alpha} \bar{v}_{h}^{\alpha}=\left(p_{h} u_{h}, p_{h} v_{h}\right) \\ \left(\left(u_{h}, v_{h}\right)_{* h}=\sum_{\alpha=1}^{n} \lambda_{\alpha}^{-1} \dot{u}_{h}^{\alpha} \bar{v}_{h}^{\alpha}=\left(\left(p_{h} u_{h}, p_{h} v_{h}\right)_{*}\right.\right.\end{array}\right.$

Posons, si $\mathrm{h}=\mathrm{n}^{-1}$ :

(1-34) $\quad p_{h} u_{h}=\sum_{\alpha=1}^{n} u_{h}^{\alpha} \omega^{\alpha} ; r_{h}^{*} u=\left(\left(u, w^{\alpha}\right)\right)_{1 \leqslant \alpha \leqslant n}$

PROPOSITION 1-5.

Sous $\ell$ 'hypothese (1-31) les approximations : 
$A_{h}(V)=\left(V, v_{h}, p_{h}, r_{h}^{*}\right) ; A_{h}(H)=\left(H, H_{h}, p_{h}, r_{h}^{*}\right) ; A_{h}\left(V^{\prime}\right)=\left(V^{\prime}, V_{h}^{\prime}, p_{h}, r_{h}^{*}\right)$

sont des approximations autoadjointes stables et convergentes des espaces $\mathrm{V}, \mathrm{H}$ et $V^{\prime}$.

En effet, $\frac{\omega^{\alpha}}{\sqrt{\lambda_{\alpha}}}$ est une base orthonormale de $v$ et $\sqrt{\lambda_{\alpha}} \omega^{\alpha}$ est une base orthonormale de $V^{\prime}$, (d'après $(1-32)$ et $(1-33)$ ). La proposition résulte de ce que :

$p_{h} r_{h}^{*} u=\sum_{\alpha=1}^{n}\left(u, \omega^{\alpha}\right) \omega^{\alpha}=\sum_{\alpha=1}^{n}\left(\left(u, \frac{\omega^{\alpha}}{\sqrt{\lambda_{\alpha}}}\right) \frac{\omega^{\alpha}}{\sqrt{\lambda_{\alpha}}}=\sum_{\alpha=1}^{n}\left(\left(u, \sqrt{\lambda_{\alpha}} \omega^{\alpha}\right)\right)_{*} \sqrt{\lambda_{\alpha}} \omega^{\alpha}\right.$

et des propriétés des bases orthonormales d'un espace Hilbertien.

En particulier, les troncatures $\mathrm{p}_{h} \mathrm{r}_{h}{ }^{*}$ sont des projecteurs des espaces $V$, H , V' - Ces approximations de Galerkine autoadjointes sont "optimales" (J P AUBIN [4]).

1-9 TRONCATURES DE TCHEBYCHEV.

Nous allons considérer dans ce numéro des restrictions $r_{h}$ telles que les troncatures soient des projections sur un ensemble $p_{h} K_{h}$.

Donnons-nous deux espaces de Banach $E$ et $E_{h}$ et un prolongement $p_{h}$ (1inéaire continu) de $E_{h}$ dans $E$.

Supposons que :

(1-35) $E_{h}$ est réflexif; $K_{h}$ est un sous ensemble convexe fermé de $E_{h}$. PROPOSITION 1-6.

Il existe au moirs une restriction $r_{h}$ de $E$ dans $k_{h}$ telle que

(1-36) $\quad\left\|u-p_{h} r_{h} u\right\| \leqslant\left\|u-p_{h} u_{h}\right\|$ pour tout $u_{h}$ de $k_{h}$.

DEFINITION 1-7.

Si (1-36) a lieu, nous dirons que $p_{h} r_{h}$ (resp. $r_{h}$ ) est une troncature (resp. une restriction) de Tchebychev ou de meilleure approximation dans $k_{h}$.

Considérons la fonctionnelle $J_{u}\left(u_{h}\right)=\left\|u-p_{h} u_{h}\right\|$. Elle est faiblement semicontinue inférieurement sur $E_{h}$. D'après le théorème de Weirstrass, elle atteint son minimum en un point $r_{h} u$ d'un ensemble $B_{h}$ si celui-ci est faiblement compact.

Solt $s_{h} u$ un élément particulier de $K_{h}$. Alors $J_{u}\left(s_{h} u\right) \geqslant n$, où :

$$
n=\inf _{k_{h}} J_{u}\left(u_{h}\right) \text {. }
$$


Donc si ||$p_{h} u_{h}-p_{h} s_{h} u|| \geqslant 2 J_{u}\left(s_{h} u\right)$, alors:

$J_{u}\left(u_{h}\right)=\left\|u-p_{h} s_{h} u+p_{h} s_{h} u-p_{h} u_{h}\right\|>n$.

Donc si le minimum $r_{h} u$ atteint par $J_{u}\left(u_{h}\right)$ sur $k_{h}$ existe, 11 appartient nécessairement à l'intersection $B_{h}$ de $K_{h}$ et de l'image réciproque par $p_{h}$ de la boule fermée de centre $P_{h} s_{h} u$ et de rayon $2 J_{u}\left(s_{h} u\right)$. L'ensemble $B_{h}$ est alors convexe, fermé et borné, donc faiblement compact puisque $E_{h}$ est réflexif, ce qui implique que le minimum $r_{h} u$ existe. En particulier, si $K_{h}=E_{h}$ est un espace de dimension finie, l'hypothèse (1-35) est satisfaite et 11 existe une restriction de Tchebychev.

REMARQUE $1-2$.

S'11 existe des approximations $A_{h}(E)=\left(E, E_{h}, p_{h}, s_{h}\right)$ convergentes de $E$ et si $P_{h} K_{h} \subset K$, les approximations de Tchebychev $A_{h}(K)=\left(K, K_{h}, P_{h}, r_{h}\right)$ de $K$ sont convergentes.

En particulier, si : $\mathrm{p}_{h} K_{h} \subset p_{k} K_{k}$ pour $k<h$ et si $\bigcup_{h} p_{h} K_{h}$ est dense dans $K$, les approximations $A_{h}(K)$ de Tchebychev sont convergentes.

REMARQUE 1-3.

En adaptant un résultat classique, on montre que si la norme de $E$ est strictement convexe :

$\|u+v\|=\|u\|+\|v\|$ si et seulement si $u=\lambda v(u, v \neq 0) ;$ la restriction de Tchebychev est unique (si $k_{h}$ est convexe).

1-10. CONSTRUCTION D'APPROXIMATIONS.

Soit $A_{h}(E)=\left(E, E_{h}, P_{h}, r_{h}\right)$ une approximation de $E$.

Donnons-nous un espace $F$ et des applications linéaires continues $P_{h}$ et $R_{h}$ : (1-37) $\quad P_{h} \in L(E, F) ; R_{h} \varepsilon L(F, E)$.

Il est clair que :

(1-38) $\quad A_{h}(F)=\left(F, E_{h}, P_{h} P_{h}, r_{h} R_{h}\right)$ est une approximation de $F$ dans $\mathrm{E}_{\mathrm{h}}$.

Si l'approximation $A_{h}(E)=\left(E, \sigma_{h}, S_{h}\right)$ est une approximation discrète $\left(n^{\circ} 1-7\right), 1^{\prime}$ approximation $A_{h}(F)$ est également discrète :

$A_{h}(F)=\left(F, P_{h} \sigma_{h}, R_{h}^{\prime} S_{h}\right)$ 
où $P_{h} \sigma_{h}=\left(P_{h} \sigma_{h}^{\alpha}\right)_{\alpha \in R_{h}}$ et $R_{h}^{\prime} s_{h}=\left(R_{h}^{\prime} s_{h}^{\alpha}\right)_{\alpha \varepsilon R_{h}}$

PROPOSITION 1-7.

Supposons que les approximations $A_{h}(E)$ soient stables et convergentes. Si $P_{h}$ et $R_{h}$ convergent simplement vers des opērateurs $P$ et $R$ et vérifient :

$(1-39)\left\{\begin{array}{l}\text { i) }|| P_{h} u\left\|_{F} \leqslant\right\| u\left\|_{E} ;\right\| R_{h} v\left\|_{E} \leqslant\right\| v \|_{F} \\ \text { ii) } \quad P R=1\end{array}\right.$

les approximations $A_{h}(F)$ sont également stables et convergentes.

REMARQUE 1-4.

Les restrictions $r_{h}$ ne sont pas supposées être nécessairement linéaires. La stabilité des approximations $A_{h}(F)$ est évidente d'après (1-39) i).

La condition (1-39) i) exprime que les opérateurs $P_{h}$ et $R_{h}$ forment des ensembles (uniformément) équicontinus $\mathrm{H}_{1}$ et $\mathrm{H}_{3}$ de $\mathrm{C}(\mathrm{E}, \mathrm{F})$ et $\mathrm{C}(\mathrm{F}, \mathrm{E})$, et la stabilité des approximations $A_{h}(E)$ entrafine que les troncatures $p_{h} r_{h}$ forment un ensemble équicontinu $\mathrm{H}_{2}$ de $\mathrm{C}(\mathrm{E}, \mathrm{F})$. La composition des fonctions étant continue sur les ensembles équicontinus (munis de la convergence simple), on en déduit que les troncatures $P_{h} P_{h} r_{h} R_{h}$ de $A_{h}(F)$ convergent simplement vers $P R=1$ dans $C(F, F)$, ce qui achève la démonstration de la proposition.

La condition (1-39) ii) exprime que $F$ est isomorphe à un sous-espace fermé de E.

La proposition 1-7 permet de déduire d'une famille d'approximations la construction d'autres approximations.

Remarquons que $1^{\prime}$ approximation duale de $A_{h}(F)$ est :

$(1-40) \quad A_{h}^{*}\left(F^{\prime}\right)=\left(F^{\prime}, E_{h}^{\prime}, R_{h}^{\prime} p_{h}^{*}, r_{h}^{*} P_{h}^{\prime}\right)$

REMARQUE $1-5$.

On déduit de la proposition 1-2 que si $P R=1$, si $R$ est compact et si les approximations $A_{h}(E)$ sont stables et convergentes, les approximations duales $A_{h}^{*}\left(F^{\prime}\right)$ sont stables et (fortement) convergentes. 
§2. APPROXIMATIONS DES OPERATEURS.

On définit au $n^{\circ}$ 2-1 la consistance des opérateurs approchés, aux $n^{\circ} 2-2$ et 2-3 la stabilité des opérateurs approchés. Le $n^{\circ} 2-4$ étudie les conditions suffisantes pour que la convergence discrète des données implique celle des solutions (Théorème 2-1). (Ces résultats peuvent se généraliser au cas où $E$ est un espace topologique complètement régulier).

Le $\mathrm{n}^{\circ}$ 2-5 étudie les conditions suffisantes pour que la convergence faible des données implique la convergence faible des solutions, le $n^{\circ} 2-6$ ' $l^{\prime}$ approximation des opérateurs compacts.

On montre au $n^{\circ}$ 2-7 que la stabilité des différentielles de Fréchet d'une famille d'opérateurs entraÍne la stabilité "locale" de ces opérateurs.

Le théorème 2-1 sera utilisé constamment dans ce chapitre. On suppose connus les $\mathrm{n}^{\circ} 1-1$ à $1-3$ du $\delta 1$.

2-1 APPROXIMATIONS DES ESPACES D'OPERATEURS.

Donnons-nous deux familles d'approximations :

1) $A_{h}(E)=\left(E, E_{h}, p_{h}, r_{h}\right)$

11) $A_{h}(F)=\left(F, F_{h}, q_{h}, s_{h}\right)$

des espaces de Banach $E$ et $F$. Solent $F(E, F)$ et $F\left(E_{h}, F_{h}\right)$ les espaces d'opérateurs de $E$ dans $F$ et de $E_{h}$ dans $F_{h}$. On définit des prolongements $P_{h}$ de $F\left(E_{h}, F_{h}\right)$ dans $F(E, F)$ et des restrictions $R_{h}$ de $F(E, F)$ dans $F\left(E_{h}, F_{h}\right)$ de la manière suivante :

i) $P_{h} A_{h}=q_{h} A_{h} r_{h} \quad ; A_{h} \in F\left(E_{h}, F_{h}\right)$

ii) $R_{h} A=s_{h} A p_{h} \quad ; A \in F(E, F)$

comme le montre le schéma suivant :

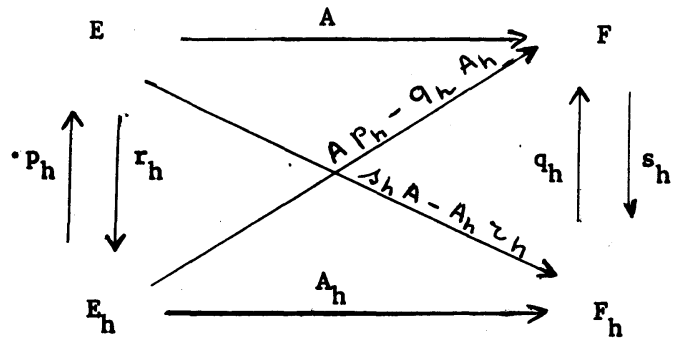


DEFINITION 2-1.

Nous dirons qu'une famille d'opérateurs $A_{h}$ de $E_{h}$ dans $F_{h}$ est consistante $\bar{a}$ un opérateur $\mathrm{A}$ de $\mathrm{E}$ dans $\mathrm{F}$ sur $\mathrm{B} C \mathrm{E}$ si :

(2-3) $\lim _{h=0}\left\|s_{h} A(u)-A_{h}\left(r_{h} u\right)\right\|_{F_{h}}=0$ pour tout $u$ de $B$.

Nous dirons qu'une famille d'opérateurs $A_{h}$ es.t (faiblement) ajustable à $A$ si :

(2-4) ||$p_{h} u_{h} \| \leqslant M$ entraine que $\underset{h=0}{\lim } A\left(p_{h} u_{h}\right)-q_{h} A_{h}\left(u_{h}\right)=0$ dans $E$ (jaible).

2-2 LES ESPACES $\pi E_{h}$ et $\beta E_{h}$ ASSOCIES A $A_{h}(E)$.

Donnons-nous une famille d'approximations $A_{h}(E)$ d'un espace de Banach dans des espaces de Banach $E_{h}$, munis des normes $\left\|u_{h}\right\|_{h}$. Nous avons été conduits jusqu' ici à considérer des familles $\left(u_{h}\right)_{h}$ d'éléments $u_{h}$ de $E_{h}$, et ces familles $\left(u_{h}\right)_{h}$ appartiennent à l'espace produit $\underset{h \in Z}{\pi} E_{h}$.

Nous allons introduire sur cet espace produit une topologie plus fine que la topologie produit.

Pour simplifier les notations, nous désignerons par

(2-5) $\quad \pi u_{h}=\left(u_{h}\right)_{h \varepsilon Z} \quad \varepsilon \prod_{h} E_{h}$

une famille d'éléments $u_{h}$ de $E_{h}$.

DEFINITION 2-2.

Nous désignerons par $\pi E_{h}$ l'espace métrique obtenu en munissant l'espace produit II $\mathrm{E}_{\mathrm{h}}$ de la distance :

(2-6) $\quad\left\|\pi u_{h}-\pi v_{h}\right\|=\sup _{h \in Z}\left\|u_{h}-v_{h}\right\|_{h}$

Nous désignerons par $B \mathrm{E}_{\mathrm{h}}$ le sous espace des $\pi u_{h}=B u_{h}$ tels que

(2-7) $\quad\left\|B u_{h}\right\|=\sup _{h \in Z}\left\|u_{h}\right\|_{h}<+\infty$

et nous dirons que la famille $\beta u_{h}$ est stable.

PROPOSITION 2-1.

Si les espaces $E_{h}$ sont des espaces de Banach, l'espace $\pi E_{h}$ est un groupe 
métrique complet et l'espace $B E_{h}$ un espace de Banach.

Il est clair que l'injection de $\pi E_{h}$ dans $\pi E_{h}$ est continue. Montrons que tout voisinage d'un système fondamental de voisinages de l'origine de $\pi E_{h}$ est fermé dans $\Pi E_{h}$. Mais les produits $\Pi B_{h}(\varepsilon)$ des boules fermées de rayon $\varepsilon$ forment un système fondamental de voisinages de $\pi E_{h}$, fermés dans $\pi E_{h}$. On sait alors que, puisque $\Pi E_{h}$ est complet, il en est de même de $\pi E_{h}$.

Montrons maintenant que $B E_{h}$ est fermé dans $\pi E_{h}$. Soit $\pi u_{h}$ une famille adhérente à $B E_{h}$, et $\varepsilon>0$. Il existe $\beta v_{h}$ tel que $\left\|\pi u_{h}-\beta v_{h}\right\| \leqslant \varepsilon$. Alors :

$$
\left\|\pi u_{h}\right\| \leqslant\left\|\pi u_{h}-\beta v_{h}\right\|+\left\|\beta v_{h}\right\|<+\infty
$$

ce qui implique que $\pi u_{h}=\beta u_{h} \varepsilon \beta E_{h}$.

EXEMPLE 2-1.

Si tous les espaces $E_{h}$ sont égaux à $E, \pi E$ est l'espace des fonctions $u_{h}$ de $Z$ dans $E$ muni de la topologie de la convergence uniforme sur $Z$. L'espace $B E$ est alors le sous-espace de Banach des fonctions bornées de $Z$ dans $E$.

REMARQUES $2-1$.

Si $B(Z)$ est 1 'espace des fonctions scalaires $\lambda(h)$ bornées sur $Z, \pi E_{h}$ est un $B(Z)$ module topologique $\left(\lambda . \pi u_{h}=\pi \lambda(h) u_{h}\right)$.

L'espace $B E_{h}$ est ouvert dans $\pi E_{h}$. Si l'on modifie les normes de $E_{h}$, on obtient des espaces $B E_{h}$ différents. (Cf. remarque $1-1$ du $\$ 1, n^{0} 1-1$ ).

2-3 STABILITE DES OPERATEURS.

Donnons des approximations $A_{h}(E)$ et $A_{h}(F)$ des espaces $E$ et $F$ et considérons les espaces $\pi E_{h}$ et $\pi F_{h}$ associés.

Soit $\left(A_{h}\right)_{h}$ une famille d'opérateurs $A_{h}$ de $E_{h}$ dans $F_{h}$. Elle définit un opérateur $\pi A_{h}$ de $\pi E_{h}$ dans $\pi F_{h}$ de la manière suivante :

(2-8) $\quad \pi A_{h}\left(\pi u_{h}\right)=\pi A_{h}\left(u_{h}\right)$

DEFINITION 2-3.

Nous dirons que les opérateurs $A_{h}$ de $E_{h}$ dans $F_{h}$ sont stables si: 
(2-9) $\quad \pi A_{h}$ est uniformément continu de $\pi E_{h}$ dans $\pi F_{h}$ c'est-à-dire si :

(2-9) $\left\{\begin{array}{l}\text { Pour tout } \varepsilon>0, \text { il existe } n \text { tel que } \\ \sup _{h}\left\|u_{h}-v_{h}\right\|_{E_{h}} \leqslant n \text { entraine que } \underset{h}{\sup }\left\|A_{h}\left(u_{h}\right)-A_{h}\left(v_{h}\right)\right\|_{F_{h}} \leqslant \varepsilon\end{array}\right.$

Nous dirons que les opérateurs $A_{h}$ sont globalement bornēs si:

(2-10) $\pi A_{h}$ est un opérateur borné de $B E_{h}$ dans $B F_{h}$

c'est-à-dire si :

(2-10) $\quad \sup _{h}\left\|u_{h}\right\|_{E_{h}} \leqslant M$ entraine que $\sup _{h}\left\|A_{h}\left(u_{h}\right)\right\|_{F_{h}} \leqslant M^{\prime}$

Cette notion est conforme au sens intuitif. Si les erreurs commises entre $u_{h}$ et $v_{h}$ sont "bornées" en $h$, il en est de même des erreurs commises entre $A_{h}\left(u_{h}\right)$ et $A_{h}\left(v_{h}\right)$.

PROPOSITION 2-2.

Si les opérateurs $A_{h}$ sont linēaires, les conditions (2-9) et (2-10) sont toutes les deux équivalentes à :

(2-11) Il existe $M>0$ telle que $\left\|A_{h} u_{h}\right\|_{F_{h}} \leqslant M|| u_{h} \|_{E_{h}}$ pour tout $h$.

I1 est clair que (2-11) implique (2-9) et (2-10). Inversement, (2-10) implique (2-11) puisque $\pi A_{h}$ est 1 opérateur linéaire borné de $B E_{h}$ dans $B F_{h}$, donc continu d'après la proposition 2-1. Montrons enfin que la stabilité entraine (2-13). Dire que $\pi \mathrm{A}_{\mathrm{h}}$ est continu à 1 'origine est dire que pour tout a $>0,11$ existe $b>0$ tel que

(2-12) $\sup _{h}\left\|v_{h}\right\|_{E_{h}} \leqslant b$ entraine que $\sup _{h}\left\|A_{h} v_{h}\right\|_{F_{h}} \leqslant a$.

Soit $\pi u_{h}$ un élément arbitraire de $\pi E_{h}$. Posons :

$\lambda(h)=b|| u_{h} \|_{E_{h}}^{-1}$ si $u_{h} \neq 0 ; \lambda(h)=1$ si $u_{h}=0$.

alors $\pi v_{h}=\pi \lambda(h) u_{h}$ vérifie 1'hypothèse de (2-12) et par suite:

(2-11) $\left\|A_{h} u_{h}\right\|_{F_{h}} \leqslant a \lambda(h)^{-1}=\frac{a}{b}\left\|u_{h}\right\|_{E_{h}}$ 
REMARQUE 2-2.

Les définitions 1-2 du $\$ 1$ sont compatibles avec la définition 2-3. Dire que les prolongements sont stables est dire que $\pi P_{h}$ est continu de $\pi E_{h}$ dans $\pi E$ et dire que les restrictions sont globalement bornées est dire que $\pi r_{h}$ est continu de $\pi \mathrm{E}$ dans $\pi \mathrm{E}_{\mathrm{h}}$.

2-4. APPROXIMATION DISCRETE DES OPERATEURS.

Considérons les problèmes suivants :

PROBLEMES Q(resp. $\left.Q_{h}\right)$

Etant donne $u\left(\right.$ resp. $u_{h}$ ) dans $E$ (resp. $E_{h}$ ), obtenir $f=A(u)$ (resp. $f_{h}=$ $A_{h}\left(u_{h}\right)$ dans $F\left(\right.$ resp. $\left.F_{h}\right)$

PROBLEMES P(resp. $\mathrm{P}_{\mathrm{h}}$ )

Etant donne $f$ dans $F$ (resp. $f_{h}$ dans $F_{h}$ ), trouver $u$ dans $E$ (resp. $u_{h}$ dans $E_{h}$ ) tels que $A(u)=f$ (resp. $\left.A_{h}\left(u_{h}\right)=f_{h}\right)$.

Les résultats généraux suivants établissent des conditions sur les opérateurs $A_{h}$ pour que la convergence discrète des données entraîne celle des solutions.

LEMME 2-1.

Pour que la convergence discrète de $u_{h}$ vers $u$ entraine celle de $f_{h}=A_{h}\left(u_{h}\right)$ vers $\mathbf{f}=\mathrm{A}(\mathrm{u})$, il suffit que:

(2-13) $\left\{\begin{array}{l}\text { i) les opérateurs } A_{h} \text { soient consistants } \bar{a} A . \\ \text { ii) les opérateurs } A_{h} \text { soient stables. }\end{array}\right.$

Ces conditions sont nécessaires si les opérateurs $A_{h}$ et $A$ sont linéaires.

Les conditions sont suffisantes. En effet :

(2-14) $\quad f_{h}-s_{h} f=A_{h}\left(u_{h}\right)-A_{h}\left(r_{h} u\right)+A_{h}\left(r_{h} u\right)-s_{h} A(u)$.

Pour tout $\varepsilon$, 11 existe $n$ tel que

$\left\|A_{h}\left(u_{h}\right)-A_{h}\left(r_{h} u\right)\right\|_{F_{h}} \leqslant \frac{\varepsilon}{2}$ dès que $\left\|u_{h}-r_{h} u\right\|_{E_{h}} \leqslant n$

d'après la stabilité. La convergence discrète de $u_{h}$ vers $u$ et la consistance des opérateurs $A_{h}$ à $A$ implique que pour $h \leqslant h_{0}$ 
$\left\|u_{h}-r_{h} u\right\|_{E_{h}} \leqslant n \quad$ et $\quad\left\|A_{h}\left(r_{h} u\right)-s_{h} A(u)\right\|_{F_{h}} \leqslant \frac{\varepsilon}{2}$.

On déduit de tout ceci que $\left\|f_{h}-s_{h} f\right\|_{F_{h}} \leqslant \varepsilon$ pour $h$ assez petit.

Les conditions sont nécessalres si $A_{h}$ et $A$ sont linéaires :

Tout d'abord, les opérateurs $A_{h}$ sont stables, sinon, 11 existerait $B u_{h}$ dans $B E_{h}$ tels que :

$$
\left\|u_{h}\right\|_{E_{h}} \leqslant M \text { et } n_{h}=\left\|A_{h} u_{h}\right\|_{F_{h}} \text { tend vers l'infin1. }
$$

L'hypothèse est contredite en prenant $v_{h}=n^{-1 / 2} u_{h}$, qui converge discrètement vers 0 , tandis que $\left\|A_{h} v_{h}\right\|_{F_{h}}$ tend vers l'infini.

Les opérateurs $A_{h}$ étant stables, on déduit de (2-14) que les opérateurs $A_{h}$ sont consistants à A .

THEOREME 2-1.

Supposons que les opérateurs $A_{h}$ et $A$ soient bijectifs. Pour que la convergence discrète de $f_{h}$ vers $f$ entraine celle de $u_{h}=A_{h}^{-1}\left(f_{h}\right)$ vers $u=A^{-1}(f)$, il subfit que :

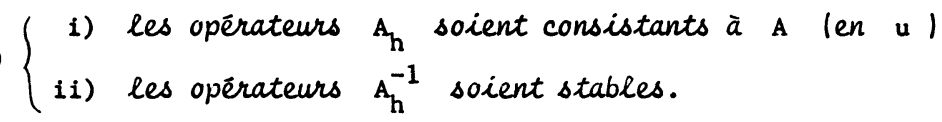

Ces conditions sont nécessaires si les opérateurs $A_{h}$ et $A$ sont linéaires et si les opérateurs $A_{h}$ sont stables.

D'après le lemme précédent, il suffit de montrer que la consistance des opérateurs $A_{h}$ à $A$ implique la consistance des opérateurs $A_{h}^{-1}$ à $A^{-1}$, si les opérateurs $A_{h}^{-1}$ sont stables.

Mais cela résulte immédiatement de :

(2-16) $r_{h} A^{-1}(f)-A_{h}^{-1}\left(s_{h} f\right)=A_{h}^{-1}\left(A_{h}\left(r_{h} u\right)\right)-A_{h}^{-1}\left(s_{h} A(u)\right)$

Inversement, si les $A_{h}$ sont linéaires, le lemme précédent entraíne que les opérateurs $A_{h}^{-1}$ sont stables et consistants à $A^{-1}$. Le raisonnement précédent montre que si les $A_{h}$ sont stables, les opérateurs $A_{h}$ sont consistants à $A$.

Nous allons donner maintenant des exemples d'opérateurs $A_{h}$ stables et consistants à A : 
PROPOSITION 2-3.

Supposons que l'opèrateur A soit uniformément continu et que (i) les prolongements $p_{h}$ et les restrictions $s_{h}$ soient stables (ii) Les approximations $A_{h}(E)$ soieint convergentes.

Alors les restrictions $s_{h} A P_{h}$ de l'opërateur A sont stables et consistantes $\bar{a}$ A .

En effet, puisque $\pi s_{h} A p_{h}=\pi s_{h} \cdot \pi A . \pi p_{h}$. est le produit de trois opérateurs uniformément continus, il est lui-même uniformément continu, ce qui montre la stabilité des restrictions $s_{h} A p_{h}$.

D'autre part, $\| s_{h} A\left(p_{h} r_{h} u\right)-\left.s_{h} A(u)\right|_{F_{h}}<\varepsilon$ donné dès que $\left\|\dot{p}_{h} r_{h} u-u\right\|<n$ puisque $\pi s_{h} A$ est uniformément continu, et cette dernière égalité est réalisée pour $h$ assez petit puisque les approximations $A_{h}(E)$ sont convergentes. Les restrictions $s_{h} A p_{h}$ de A sont bien consistantes à $A$.

Nous donnerons au $\$ 3 n^{\circ} 3.3$ des exemples d'opérateurs $A$ inversibles tels que leurs restrictions soient inversibles et stables.

REMARQUES 2-3.

Dans le cas où les opérateurs $A_{h}$ sont linéalres, le théorème 1 du $\$ 2$ est un résultat classique : cf. par exemple S. GODUNON - V. RYABENKII , [1], V. THOMEE, $[1]$, P.D. LAX [1] , etc...

Si les opérateurs $\pi A_{h}^{-1}$ est Höldérien :

$$
\left\|A_{h}^{-1}\left(f_{h}\right)-A_{h}^{-1}\left(g_{h}\right)\right\|_{h} \leqslant M|| f_{h}-g_{h} \|_{h}^{\theta} ; \quad M, \theta>0 \text {. }
$$

Nous en déduisons une évaluation de l'erreur discrète :

(2-18) $\quad \mid u_{h}-r_{h} u \|_{h} \leqslant M\left(|| f_{h}-s_{h} f\left\|_{h}+\right\| s_{h} A(u)-A_{h} r_{h}(u) \|_{h}\right)^{\theta}$

Si les opérateurs $A_{h}$ ne sont pas linéaires, la condition de stabilité n'est pas nécessaire.

Nous montrerons au $n^{\circ} 2-7$ (théorème 2 du \$2) que si les opérateurs $A_{h}$ sont différentiables, la stabilité des inverses des différentielles en $r_{h} u_{0}$ entraîne la stabilité locale des opérateurs $A_{h}^{-1}$.

Nous donnerons aux $\$ 3\left(n^{\circ} 3-2\right)$ deux conditions suffisantes de stabilité lorsque les opérateurs sont définis d'un espace de Banach $V$ dans son dual. 
Remarquons enfin que la théorie classique de l'approximation des solutions des équations différentielles usuelles entre dans le cadre de la théorie précédente : le théorème 1 du $\$ 2$ implique en particulier le théorème classique affirmant que stabilité et consistance entraînent la convergence discrète des solutions. (Pour des conditions de stabilité plus faibles, cf. J.P. AUBIN [5] .).

2-5. APPROXIMATION FAIBLE DES OPERATEURS.

Nous allons étudier les conditions suffisantes pour que la convergence faible des données des problèmes $P_{h}$ et $Q_{h}$ entraîne la convergence faible des solutions.

Nous dirons qu'un opérateur A est faiblement continu de E dans F (resp. uniformément faiblement continu) si A est continu (resp. uniformément continu) de $E$ faible dans $F$ faible. Tout opérateur linéaire continu d'un espace de Banach E dans un espace de Banach $F$ est uniformément faiblement continu.

LEMME 2-2.

Supposons que A soit faiblement continu. Pour que la convergence faible de $u_{h}$ vers $u$ entraine la convergence faible de $f_{h}=A_{h}\left(u_{h}\right)$ vers $f=A(u)$, il subfit que $A_{h}$ soit faiblement ajustable à $A$. Cette condition est nécessaire si E est réflexif.

La condition suffisante résulte de l'inégalité :

$$
f-q_{h} f_{h}=A(u)-A\left(p_{h} u_{h}\right)+A\left(p_{h} u_{h}\right)-q_{h} A_{h}\left(u_{h}\right)
$$

puisque, si $p_{h} u_{h}$ converge faiblement vers $u,\left\|p_{h} u_{h}\right\|$ est borné.

Inversement, si $E$ est réflexif (ou est le dual d'un espace de Banach) et si $\left\|p_{h} u_{h}\right\|$ est borné, 11 existe (au moins) $u^{*}$ faiblement adhérent à $p_{h} u_{h}$. Donc si une sous-suite $p_{h_{n}} u_{h_{n}}$ converge faiblement vers $u_{*}, q_{h_{n}} A_{h_{n}}\left(u_{h_{n}}\right)$ converge faiblement vers $A\left(u^{*}\right)$ et 0 est faiblement adhérent à $A\left(p_{h} u_{h}\right)-q_{h} A_{h}\left(u_{h}\right)$ et c'est le seul point faiblement adhérent (d'après (2-19)).

LEMME 2-3.

Supposons que $l^{\prime}$ inverse $\mathrm{A}^{-1}$ de $\mathrm{A}$ soit uniformément faiblement continu et que (2-20) $\left\{\begin{array}{l}\text { i) les prolongements } \mathrm{p}_{\mathrm{h}} \text { sont stables } \\ \text { ii) les opérateurs } \mathrm{A}_{h}^{-1} \text { sont globalement bornés } \\ \text { iii) les opérateurs } A_{h} \text { sont faiblement ajustables } \tilde{a} \text { A. }\end{array}\right.$ Si $\left\|f_{h}\right\|_{F_{h}} \leqslant M$ et si $f_{h}$ converge faiblement vers $f, u_{h}=A_{h}^{-1}\left(f_{h}\right)$ 
converge faiblement vers $u=A^{-1}(f)$.

Le lemme résulte de l'égalité :

(2-21) u- $p_{h} u_{h}=\left[A^{-1}(f)-A^{-1}\left(q_{h} f_{h}\right)\right]+\left[A^{-1}\left(q_{h} A_{h}\left(u_{h}\right)\right)-A^{-1}\left(A\left(p_{h} u_{h}\right)\right)\right]$

Si $q_{h} f_{h}$ converge faiblement vers $f$, le premier terme du membre de droite tend vers 0 . SI $\left\|f_{h}\right\|_{F_{h}} \leqslant M,\left\|u_{h}\right\|_{E_{h}} \leqslant M$ puisque les opérateurs $A_{h}^{-1}$ sont globalement bornés, donc $\left\|\mathrm{p}_{\mathrm{h}} u_{\mathrm{h}}\right\|$ est borné puisque les prolongements sont stables. Le second terme du membre de droite tend vers 0 puisque les $A_{h}$ sont faiblement ajustables à $A$ et puisque $A^{-1}$ est uniformément faiblement continu.

Nous allons donner des exemples d'opérateurs $A_{h}$ faiblement ajustables à A :

PROPOSITION 2-4.

Supposons que l'opérateur A soit borné, que F soit réflexif et que les approximations duales $A_{h}^{*}\left(F^{\prime}\right)$. soient convergentes. Les restrictions $s_{h} A p_{h}$ de l'opérateur A sont faiblement ajustables à A.

Il faut montrer que si $\left\|p_{h} u_{h}\right\|$ est borné, $\left(1-q_{h} s_{h}\right) A\left(p_{h} u_{h}\right)$ converge faiblement vers 0 .

Mais $A\left(p_{h} u_{h}\right)$ est dans un borné de $F$ puisque $A$ est borné et que $q_{h} s_{h}$ converge vers 1 faiblement et uniformément sur tout ensemble borné de $F$ d'après 1a proposition 1-2 puisque les approximations duales $A_{h}^{*}\left(F^{\prime}\right)$ sont convergentes.

Nous donnerons des exemples d'approximation faible d'opérateurs non linéaires monotones et coercifs au $n^{\circ} 3-5$ du $\$ 3$ et au $n^{0} 4-3$ du $\$ 4$ du chapitre II.

\section{2-6 APPROXIMATION DES OPERATEURS COMPACTS.}

Un opérateur $A$ de $E$ dans $F$ est compact si l'image par $A$ de tout ensemble borné de $E$ est relativement compacte dans $F$. Nous allons établir des propriétés analogues pour des approximations de $A$.

PROPOSITION 2-5.

Supposons que les approximations $A_{h}(F)$ soient stables et convergentes. Les restrictions $\mathbf{s}_{h} A_{p_{h}}$ d'un opérateur compact $\mathbf{A}$ sont ajustables $\bar{a} \mathbf{A}$.

$$
\begin{aligned}
& \text { Montrons que si }\left\|p_{h} u_{h}\right\| \text { est borné : } \\
& \left(1-q_{h} s_{h}\right) A\left(p_{h} u_{h}\right) \text { converge vers } 0 \text { dans } F \text {. }
\end{aligned}
$$


Mais $A\left(P_{h} u_{h}\right)$ est dans un ensemble relativement compact de $F$ et 1 'on sait d'après la proposition 1-1 que $q_{h} s_{h}$ converge vers 1 uniformément sur tout ensemble compact de F.

PROPOSITION 2-6.

Supposons que les opérateurs $A_{h}$ soient ajustables à un opérateur compact $A$. Si $p_{h} u_{h}$ demeure dans un borné de $E$, alors $q_{h} A_{h}\left(u_{h}\right)$ demeure dans un ensemble relativement compact de $\mathrm{F}$.

Puisque $F$ est métrisable, on peut supposer que $Z$ est dénombrable. Il suffit de démontrer que pout tout $\varepsilon$, il existe une suite finle $h_{1}, \ldots, h_{n}$ telle que pour tout $h$, 11 existe $h_{i}$ vérifiant :

$$
\left\|q_{h} A_{h}\left(u_{h}\right)-q_{h} A_{h_{i}}\left(u_{h_{i}}\right)\right\| \leqslant \varepsilon
$$

Posons :

$$
\alpha(h)=q_{h} A_{h}\left(u_{h}\right)-A\left(p_{h} u_{h}\right)
$$

Fixons-nous $\varepsilon>0$. Puisque les $A_{h}$ sont ajustables à $A$, 11 existe $h_{m}$ tel que si $h \geqslant h_{m}$, on ait $\alpha(h) \leqslant \frac{\varepsilon}{3}$.

D'autre part, pour $h \geqslant h_{m}, A\left(p_{h} u_{h}\right)$ formant un ensemble relativement compact, il existe un élément $h_{i} d$ 'une suite finie $h_{m}, \ldots, h_{n}$ tel que

$$
\begin{aligned}
& \left\|A\left(p_{h} u_{h}\right)-A\left(p_{h_{i}} u_{h_{i}}\right)\right\| \leqslant \frac{\varepsilon}{3} \therefore \text { Alors : } \\
& \left\|q_{h} A_{h}\left(u_{h}\right)-q_{h_{i}} A_{h_{i}}\left(u_{h_{i}}\right)\right\| \leqslant \alpha(h)+\alpha\left(h_{i}\right)+\left\|A\left(p_{h} u_{h}\right)-A\left(p_{h_{i}} u_{h_{i}}\right)\right\| \leqslant \varepsilon
\end{aligned}
$$

et la suite finie $h_{1}, \ldots, h_{m}, \ldots, h_{n}$ répond à la question.

\section{2-7. APPROXIMATION DES OPERATEURS DIFFERENTIABLES.}

Un opérateur A d'un espace de Banach $E$ dans un espace de Banach $F$ est différentiable sur un ouvert $U$ de $E$ s'il existe des opérateurs linéaires continus $D A(u)$ de $E$ dans $F$ tels que :

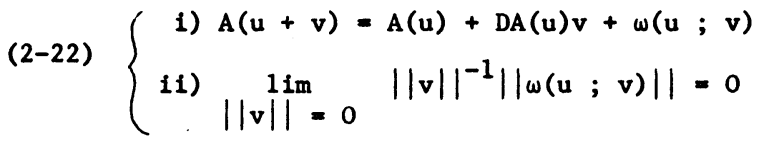

Donnons-nous maintenant des approximations $A_{h}(E)$ et $A_{h}(F)$ des espaces $E$ et $F$ et des opérateurs $A_{h}$ de $E_{h}$ dans $F_{h}$, continûment différentiables, et tels 
จิ les différentielles $\mathrm{DA} \mathrm{A}_{\mathrm{h}}\left(\mathrm{r}_{\mathrm{h}} \mathrm{u}_{\mathrm{o}}\right)$ soient inversibles pour $\mathrm{u}_{0}$ fixé dans $\mathrm{U}$. Nous alions déduire la stabiliti des opërateurs $\mathrm{A}_{\mathrm{h}}^{-1}$ dans des voisinages de $A_{h}\left(r_{h} u_{0}\right)$ de la staibilite des $\operatorname{DA}_{h}^{-1}\left(r_{h} u_{0}\right)$.

Rappelons pour cela le

LEMME (A). (M. VAINBERG $[1]$ p. 45 ).

Supposons que les dijjjérenticlies $\mathrm{u} \rightarrow \mathrm{DA}(\mathrm{u})$ soient bornées d'une boule $B(\rho+\varepsilon)$ de $E$ dars $L(E, F)$. Les dijyérentielies $u \rightarrow D A(u)$ sont uniformément continues sur $i a$ boure $B(0)$ si et semiement si :

(2-23) $\quad \lim _{\|v\|=0} \quad \sup _{\| v \rho}\|v\|^{-1}\|\omega(u ; v)\|=0$

et le :

THEORE:IE (A) (théorëme des jonctions inverses).

Soient $x$ et $y$ cieux espaces de Banach, $T$ un opérateur continüment différentiabie sur un ouvert $u$ de $x$ dans $y$. Si, pour un point $x_{0}$ de $u$, la dióóérentielie $\mathrm{DT}\left(\mathrm{x}_{0}\right)$ est un isomorpinisme de $x$ sur $y$, l'opérateur $T$ est alors un isomoriphisme d'un voisinage ouvert de $x_{0}$ sur son image.

Supposons que les opérateurs $A$ et $A_{h}$ soient continûnent différentiables, sur les boules ouvertes $U$ et $U_{h}$ de rayon $\rho+\varepsilon$ et que

(2-24) les restrictions $r_{h}$ et $s_{h}$ soient stables.

i) Si $\left\|u_{h}\right\|_{h} \leqslant K$, il existe $M$ tel que $\left\|A_{h}\left(u_{h}\right)\right\|_{h} \leqslant M$

$\begin{cases}\text { ii) Si }\left\|u_{h}\right\|_{h} \leqslant \rho+\varepsilon, & \text { il existe } M \text { tel que }\left\|D_{h}\left(u_{h}\right) v_{h}\right\|_{h} \leqslant M\left\|v_{h}\right\|_{h} \\ \text { iii) } & \left\|v_{h}\right\|_{h}=0 \quad\left\|u_{h}\right\|_{h} \leqslant \rho \\ & \left\|v_{h}\right\|_{h} \leqslant M\end{cases}$

et qu'il existe $u_{0} \varepsilon U$ tel que $r_{h} u_{0} \varepsilon U_{h}, D A\left(u_{0}\right)$ et $D_{h}\left(r_{h} u_{0}\right)$ soient inversibles et que

(2-26) $\quad\left\|D A_{h}^{-1}\left(r_{h} u_{o}\right) f_{h}\right\|_{h} \leqslant c|| f_{h} \|_{h}$ pour tout $f_{h}$ de $F_{h}$.

THEOREME 2-2.

Sous les hypothèses $(2-24),(2-25)$ et $(2-26)$, il existe $\gamma_{0}$ et $r$ 
strictement positifs tels que si:

(2-27) $\quad\left\|f-f_{0}\right\|<\gamma_{0} ; \quad\left\|f_{h}-f_{o h}\right\|_{h}<\gamma ; \quad\left(f_{o h}=A_{h}\left(r_{h} u_{o}\right)\right)$

il existe des solutions $u$ et $u_{h}$ des équations :

(2-28) $\quad \mathrm{A}(\mathrm{u})=\mathrm{f} \quad ; \quad \mathrm{A}_{\mathrm{h}}\left(\mathrm{u}_{\mathrm{h}}\right)=\mathrm{f}_{\mathrm{h}}$

uniques dans un voisinage de $u_{0}$ et de $u_{o h}$ respectivement.

Si de plus

$(2-29)\left\{\begin{array}{l}\text { i) } \lim _{h=0}\left\|s_{h} A(u)-A_{h}\left(r_{h} u\right)\right\|_{h}=0 \text { pour tout } u \in U \\ \text { ii) } \lim _{h=0}\left\|s_{h} f-f_{h}\right\|_{h}=0\end{array}\right.$

les solutions $u_{h}$ convergent aiscrètement vers la solution $u$.

DEMONSTRATION.

Nous allons montrer que l'on peut utiliser le théorème A dans la situation suivante :

$(2-30)\left\{\begin{array}{l}x=B E_{h}, y=\beta F_{h}, U=B / U_{h}, T=B A_{h} \\ x_{0}=B\left(x_{h} u_{0}\right) ; \quad f_{o h}=A_{h}\left(r_{h} u_{o}\right)\end{array}\right.$

Nous démontrerons ainsi en premier lieu que $B A_{h}$ est un isomorphisme a'un voisinage $B B_{h}$ de $B\left(r_{h} u_{0}\right)$ sur une boule $B C_{h}$ de centre $B f_{o h}$ et de rayon $\gamma>0$ convenable, ce qui impliquera en particulier que les opérateurs $A_{h}$ sont des isomorphismes de $C_{h}$ sur $B_{h}$ et que les opérateurs $A_{h}^{-1}$ sont stables sur $\mathrm{C}_{\mathrm{h}}$.

D'après (2-25) i), l'opérateur $\pi A_{h}=\beta A_{h}$ est un opérateur borné de $B E_{h}$ dans $B F_{h}$ et d'après (2-25)ii), l'opérateur $\pi D_{h}\left(B u_{h}\right)=B D A_{h}\left(B u_{h}\right)$ est un opérateur linéaire continu de $B E_{h}$ dans $B F_{h}$ d'après la proposition 2-2 du $n^{\circ} 2-3$.

De plus, l'application

$(2-31)\left\{\begin{array}{l}B u_{h} \longrightarrow \beta D_{h}\left(\beta u_{h}\right) \\ \text { est bornée de } \beta B_{h}(\rho+\varepsilon) \text { dans } L\left(\beta E_{h}, \beta F_{h}\right)\end{array}\right.$

Enfin, 1'hypothèse (2-25) iii) montre que :

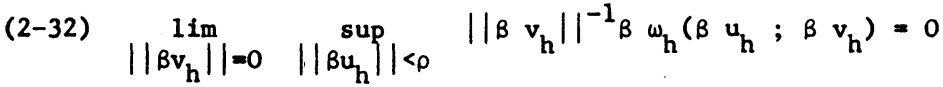

Les opérateurs $A_{h}$ étant différentiables, on déduit des relations 


$$
A_{h}\left(u_{h}+v_{h}\right)=A_{h}\left(u_{h}\right)+D A_{h}\left(u_{h}\right) v_{h}+w_{h}\left(u_{h} ; v_{h}\right)
$$

et de ce qui précède que :

(2-33) $B A_{h}\left(\beta u_{h}+\beta v_{h}\right)=\beta A_{h}\left(\beta u_{h}\right)+\beta D A_{h}\left(\beta u_{h}\right) B v_{h}+\beta w_{h}\left(\beta u_{h} ; \beta v_{h}\right)$

et que $B D A_{h}\left(B u_{h}\right)$ est la différentielle de $B A_{h}$, qui est (uniformément) continue sur $B B_{h}(\rho)$ d'après le lemme $(A),(2-31)$ et $(2-32)$.

D'autre part, l'opérateur $\pi \mathrm{DA}_{h}^{-1}\left(\mathrm{r}_{h} \mathrm{u}_{0}\right)$ est 1 'inverse (algébrique) de $\pi D A_{h}\left(r_{h} u_{0}\right)$. Mais, d'après (2-26), $\pi D_{h}^{-1}\left(r_{h} u_{0}\right)$ est continu de $B F_{h}$ sur $B E_{h}$, et par suite, la différentielle $B D_{h}\left(r_{h} u_{0}\right)$ est un isomorphisme de $B E_{h}$ sur $B F_{h}$. Il résulte alors du théorème $(A)$ que $B A_{h}$ est un isomorphisme d'un voisinage ouvert $B$ de $B r_{h} u_{0}$ sur un voisinage ouvert $C$ de $B f_{o h}=B A_{h}\left(r_{h} u_{0}\right)$.

De même, le théorème (A) appliqué dans le cas où $X=E, \quad y=F, U=U$, $T=A, x_{0}=u_{0}$ implique que $A$ est un isomorphisme d'un voisinage $B_{1}$ de $u_{0}$ sur une boule $C_{1}$ de centre $f_{0}$ et de rayon $\delta_{1}$.

La seconde partie du théorème 2-2 résulte du théorème 2-1 si 1'on montre qu'il existe des voisinages $B, B_{h}, C, C_{h}$ de $u_{0}, r_{h} u_{0}, f_{0}$ et $f_{o h}=A_{h}\left(r_{h} u_{0}\right)$ tels que : (2-34) $\left\{\begin{array}{ll}A(B)=C, & A_{h}\left(B_{h}\right)=C_{h} \\ r_{h} B \subset B_{h} & \text { et } \quad s_{h} C \subset C_{h}\end{array}\right.$;

puisque nous avons démontré que les $A_{h}^{-1}$ sont stables sur $C_{h}$ et que nous avons supposé que les $A_{h}$ sont consistants à $A$ et que les $f_{h}$ convergent discrètement vers $\mathbf{f}$.

Tout d'abord, il existe $\gamma>0$ tel que les boules $c_{h}$ de centre $f_{\text {oh }}$ et de rayon $\gamma$ vérifient $B C_{h} \subset C$, puisque les produits de boules de même rayon forment un système fondamental de voisinages de $B E_{h}$.

Considérons $B_{h}=A_{h}^{-1}\left(C_{h}\right)$. Il nous reste à montrer qu'il existe $B$ et $C$ vérifiant $(2-34)$.

Puisque les $r_{h}$ sont stables, 11 existe un voisinage $B_{2}$ de $u_{0}$ tel que $r_{h} B_{2} \subset B_{h}$, puisque la stabilité des $r_{h}$ est la continuité de l'opérateur $\pi r_{h}$ de $E$ dans $\pi E_{h}$.

Il existe alors $\delta_{2}$ tel que $0<\delta_{2} \leqslant \delta_{1}$ et $A^{-1} C\left(f_{0}, \delta_{2}\right) \subset B_{1} \cap B_{2}$. Il existe d'autre part $\delta_{3}>0$ tel que $s_{h} c\left(f_{0}, \delta_{3}\right) \subset C_{h}\left(f_{o h}, \gamma\right)$ pour $h$ assez petit. 
En effet, les $s_{h}$ étant stables, 11 existe $\delta_{3}$ tel que :

$\left\|f-f_{0}\right\| \leqslant \delta_{3}$ entraIne que $\left\|s_{h} f-s_{h} f_{o}\right\|_{h} \leqslant \frac{\gamma}{2}$

et il existe $h_{0}$ tel que, d'après $(2-29)$ i),

$\left\|s_{h} f_{0}-f_{o h}\right\|_{h}=\left\|s_{h} A\left(u_{0}\right)-A_{h}\left(r_{h} u_{0}\right)\right\|_{h} \leqslant \frac{\gamma}{2} \quad s i \quad h<h_{0}$.

Donc, en prenant pour voisinage $C$ la boule de centre $f_{0}$ et de rayon $\gamma_{0}=\inf \left(\delta_{2}, \delta_{3}\right)$ et pour voisinage $B$ l'image réciproque $A^{-1}(C)$, les conditions (2-34) sont satisfaites, ce qui achève la démonstration du théorème.

Nous donnerons au $\$ 3, n^{\circ} 3-3$, Exemple 3-6 des exemples d'opérateurs $A_{h}$ vérifiant les hypothèses du théorème 2 du $\$ 2$.

\$3. APPROXIMATION DES OPERATEURS D'UN ESPACE DANS SON DUAL.

Soit $A$ un opérateur d'un espace $V$ dans son dual $V^{\prime}$. Si on se donne une approximation $A_{h}(V)$, on prendra alors pour approximation de $V^{\prime}$ l'approximation duale de $A_{h}(V)\left(\$ 1, n^{\circ} 1-5\right)$. On fixe au $n^{\circ} 3-1$ les notations (formulation variationnelle). On donne au $n^{\circ}$ 3-2 deux conditions suffisantes commodes pour que les opérateurs $A_{h}^{-1}$ soient stables. On en déduit un théorème de convergence à 1'aide du théorème 1 du $\$ 2$. On considère ensuite 1 'application au cas des opérateurs linéaires coercifs et à une classe d'opérateurs non linéaires (exemples 3-1 et 3-2).

On montre au $n^{\circ}$ 3-3 que les restrictions d'un opérateur A sont, sous certaines conditions, consistantes à A, inversibles et que leurs inverses sont stables. On étudie de plus le comportement de l'erreur $u-p_{h} u_{h}$ dans divers espaces en fonction de l'erreur de troncature $u-p_{h} r_{h} u$.

Le $n^{\circ}$ 3-4 est consacré au cas où $A=\sum A_{i}$ est la somme d'opérateurs d'espaces $v_{i}$ dans $V^{\prime}{ }_{i}$, où $V$ est 1 'intersection des $v_{i}$. On se donne des approximations partielles des espaces $V_{i}$ et on prend pour opérateur $A_{h}$ la some des restrictions des opérateurs $A_{i}$ dans les approximations des espaces $v_{i}$. On obtient alors des résultat (moins forts) de convergence.

On considère au $n^{\circ}$ 3-5 la classe des opérateurs non linéaires, bornés, coercifs et monotones. Les conditions de stabilité ne sont pas satisfaites et nous n'obtenons qu'un théorème de convergence faible. Enfin, on considère au $n^{\circ}$ 3-6 $1^{\prime}$ approximation des solutions définies par le lemme des projections, utilisé pour démontrer l'existence des solutions des équations différentielles - opérationnelles.

On suppose connus les $n^{\circ} 1-1$ à $1-5$ du $\$ 1$ et les $n^{\circ} 2-1$ à $2-5$ du $\$ 2$. 
Les exemples illustrant les résultats de ce $\S$ sont exposés au Chapitre II , \$4, et peuvent être abordés après la lecture des $\$ 2$ et $\$ 3$ du Chapitre II .

\section{3-1. FORMULATION VARIATIONNELLE.}

Dans tout ce paragraphe, $V$ désignera un espace de Banach REFLEXIF, réel ou complexe, $V^{\prime}$ son (anti-)dual, ( $\left.f, v\right)$ le produit scalaire mettant $V$ et $V^{\prime}$ en dualité.

$\|\mathrm{u}\|$ la norme de $\mathrm{v} ;\|\mathrm{f}\|_{\star}=\underset{\mathrm{v}}{\sup }|| \mathrm{v} \|\left.\right|^{-1}|(\mathrm{f}, \mathrm{v})|$ sa norme duale.

Tout opérateur A de $\mathrm{V}$ dans $\mathrm{V}^{\prime}$ est associé biunivoquement à une forme $\mathrm{a}(\mathrm{u} ; \mathrm{v})$ sur $\mathrm{V} \times \mathrm{V}$ linéaire et continue en $\mathrm{v}$ de la manière suivante :

$(3-1) \quad a(u ; v)=(A(u), v)$.

Nous approcherons dans ce numéro les solutions du :

PROBLEME P •

Etant donné $f$ dans un sous-ensemble $\mathrm{D}$ de $\mathrm{V}^{\prime}$, chercher $\mathrm{u}$ dans un sousensemble $\mathrm{B}$ de $\mathrm{V}$ verifiant :

(3-2) $\quad a(u ; v)=(f, v)$ pour tout $v$ de $v$.

L'équation (3-2) est la formulation variationnelle de l'équation $A(u)=f$. On se donne des approximations $A_{h}(V)$ de l'espace $V$ et l'on conviendra de prendre pour approximation de $1^{\prime}$ espace $V^{\prime} 1^{\prime}$ 'approximation duale $A_{h}^{*}\left(V^{\prime}\right)$ de $A_{h}(V)$, définie par :

(3-3) $\left(p_{h}^{*} f_{h}, v\right)=\left(f_{h}, r_{h} v\right) ;\left(r_{h}^{*} f, v_{h}\right)_{h}=\left(f, p_{h} v_{h}\right) \quad\left(c f . \$ 1, n^{0} 1-5\right)$.

En particulier, nous désignerons par :

$(3-4) \quad \varepsilon\left(f, f_{h}\right)=\left\|r_{h}^{*} f-f_{h}\right\|_{* h}=\sup || v_{h} \|_{h}^{-1}\left|\left(f, p_{h} v_{h}\right)-\left(f_{h}, v_{h}\right)_{h}\right|$

l'erreur discrète entre $f$ et $f_{h}$.

Soit $A_{h}$ un opérateur de $v_{h}$ dans $V_{h}^{\prime}$ et $a_{h}\left(u_{h} ; v_{h}\right)$ sa forme associée : $(3-5) \cdot a_{h}\left(u_{h} ; v_{h}\right)=\left(A_{h}\left(u_{h}\right), v_{h}\right)_{h}$

Nous considérerons les :

PROBLEMES $\mathrm{P}_{\mathrm{h}}$.

Etant donné $\mathrm{f}_{h}$ dans un sous-ensemble $\mathrm{D}_{h}$ de $\mathrm{v}_{h}^{\prime}$, chercher $u_{h}$ dans un sous- 
ensemble $B_{h}$ de $v_{h}$ vérifiant:

$$
a_{h}\left(u_{h} ; v_{h}\right)=\left(f_{h}, v_{h}\right)_{h} \text { pour tout } v_{h} \text { de } v_{h} \text {. }
$$

Nous allons traduire sous forme variationnelle la consistance des opérateurs $A_{h}$ à un opérateur $\mathrm{A}$.

Nous poserons :

(3-7) $\quad \psi_{u}(h)=\sup _{v_{h}}|| v_{h}||_{h}^{-1}\left|a\left(u ; p_{h} v_{h}\right)-a_{h}\left(u_{h} ; r_{h} v\right)\right|$

Les formules (3-3) et(3-5) montrent que $\psi_{u}(h)=\left\|A_{h}\left(r_{h} u\right)-r_{h}^{*} A(u)\right\|_{\star h}$

Dire que les opérateurs $A_{h}$ sont consistants $\bar{a} A$ sur $B$ (ou les formes $a_{h}\left(u_{h} ; v_{h}\right)$ consistantes à la forme $a(u ; v)$ ) est dire que $\lim _{h=0} \psi_{u}(h)=0$ pour tout $u \in B$.

Nous supposerons que les opérateurs $A$ et $A_{h}$ sont surjectifs, c'est-à-dire que les problèmes $P$ et $P_{h}$ admettent des solutions. Nous allons donner des conditions suffisantes de stabilité des opérateurs $A_{h}^{-1}$ :

3-2. CONDitions Suffisantes DE STABilite des Operateurs $\mathrm{A}_{\mathrm{h}}^{-1}$.

Nous poserons pour simplifier :

$$
s_{h}\left(u_{h}, v_{h}\right)=a_{h}\left(u_{h} ; u_{h}-v_{h}\right)-a_{h}\left(v_{h} ; u_{h}-v_{h}\right)
$$

DEFINITION 3-1.

Nous dirons que les opérateurs $A_{h}$ vérifient la propriété $s_{1}$ si : $s_{1}\left\{\begin{array}{l}\text { Pour tout } \varepsilon>0, \text { il existe } n>0 \text {, indépendant de } h \text {, tel que : } \\ \left\|u_{h}-v_{h}\right\|_{h} \geqslant \varepsilon \text { entraine que }\left|s_{h}\left(u_{h}, v_{h}\right)\right| \geqslant n \mid u_{h}-v_{h} \|_{h} .\end{array}\right.$ Nous dirons que les opérateurs $A_{h}$ vérifient la propriêté $s_{2}$ si :

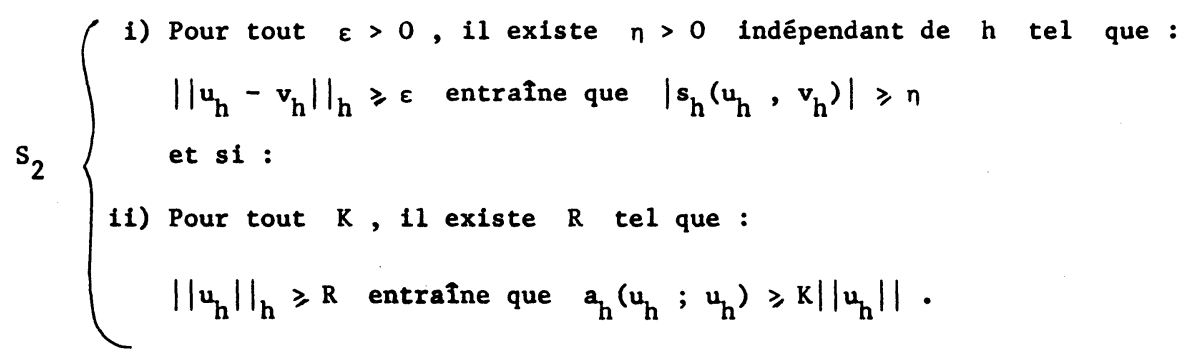


THEOREME 3-1.

Supposons les opērateurs $A$ et $A_{h}$ surjectifs. Les solutions $u$ et $u_{h}$ vèrifient :

(3-9) $s_{h}\left(u_{h}, r_{h} u\right) \leqslant\left(\psi_{u}(h)+\varepsilon\left(f, f_{h}\right)\right)\left\|u_{h}-r_{h} u\right\|$.

Si l'une des conditions $\mathrm{s}_{1}$ ou $\mathrm{S}_{2}$ est vérifiée, les opérateurs $\mathrm{A}_{\mathrm{h}}$ sont inversibles et les opérateurs $\mathrm{A}_{\mathrm{h}}^{-1}$ sont stables sur toute boule de rayon $\mathrm{K}$. Si de plus $\psi_{u}(h)$ et $\varepsilon\left(f, f_{h}\right)$ tendent vers 0 , les solutions $u_{h}$ convergent discrètement vers la solution $u$ du problème $P$. Enfin, les solutions $u_{h}$ convergent vers $u$ si les prolongements $p_{h}$ sont stables et les approximations $A_{h}(V)$ convergentes.

Les conditions $s_{1}$ et $\left.s_{2} i\right)$ impliquent que les opérateurs $A_{h}$ soient injectifs. Plus généralement, 11 suffit que si $u_{h} \neq v_{h}, s_{h}\left(u_{h}, v_{h}\right)>0$.

En effet, si on suppose que $u_{h} \neq v_{h}$ et $A_{h}\left(u_{h}\right)=A_{h}\left(v_{h}\right)$, on aurait :

$$
0=s\left(u_{h}, v_{h}\right)>0
$$

ce qui est impossible.

Montrons que les opérateurs $A_{h}^{-1}$ sont stables, c'est-à-dire que pour tout $\varepsilon$, 11 existe $n_{1}$ indépendant de $h$ tel que, si $u_{h}=A_{h}^{-1}\left(f_{h}\right)$ et $v_{h}=A_{h}^{-1}\left(g_{h}\right)$ :

(3-10) $\quad\left\|f_{h}-g_{h}\right\|_{* h} \leqslant n_{1}$ entraine que $\left\|u_{h}-v_{h}\right\|_{h} \leqslant \varepsilon$;

Supposons que $s_{1}$ soit vérifiée.Alors :

$\left|s_{h}\left(u_{h}, v_{h}\right)\right|=\left|\left(f_{h}-g_{h}, u_{h}-v_{h}\right)_{h}\right| \leqslant n_{1}|| u_{h}-v_{h}||_{h}$

ce qui implique que $\left\|u_{h}-v_{h}\right\|_{h} \leqslant \varepsilon$. (en prenant $n_{1}=n$ ).

Supposons que $S_{2}$ soit vérifiée et montrons que les $A_{h}^{-1}$ sont stables sur les boules de rayon $K$ donné dans $V_{h}^{\prime}$.

D'après $\left.S_{2} i i\right)$, on sait qu'il existe $R$ tel que si

$a_{h}\left(u_{h}, u_{h}\right)=\left(f_{h}, u_{h}\right) \leqslant\left\|f_{h}\right\|_{h}\left\|u_{h}\right\|_{h} \leqslant k\left\|u_{h}\right\|_{h}$

on ait $\left\|u_{h}\right\|_{h} \leqslant R \cdot$. Donc, pour tout $\varepsilon$, il existe $n_{1}$ tel que

$$
\left|s_{h}\left(u_{h}, v_{h}\right)\right| \leqslant n_{1} \mid u_{h}-v_{h} \|_{h} \leqslant 2 n_{1} R=n
$$

ce qui implique, d'après $\left.s_{2} i\right)$, que $\left\|u_{h}-v_{h}\right\|_{h} \leqslant \varepsilon$

Les autres assertions du théorème découlent alors du théorème 1 du $\$ 2$ du $n^{\circ} 2-4$ du 52 . 
L'inégalité (3-9) est évidente : on ajoute et on retranche à $s_{h}\left(u_{h}, r_{h} u\right)$ l'expression $a\left(u ; p_{h} v_{h}\right)=\left(f, p_{h} v_{h}\right)$.

Enfin, la dernière partie du théorème résulte du théorème 1 du $\$ 1$.

REMARQUE 3-1.

La condition $S_{2}{ }^{1 i}$ ) implique en particulier que les opérateurs $A_{h}$ sont surjectifs (cf. J. LERAY - J.L. LIONS [l] par exemple), si les espaces $v_{h}$ sont de dimension finie. Si la condition $S_{2}$ est satisfaite, et si l'opérateur $A$ est borné et continu de $V$ fort dans $V^{\prime}$ faible, on en déduit que l'opérateur $A$ est bijectif (cf. $n^{0}$ 3-4, théorème 4 du $\$ 3$ ).

L'opérateur $A$ est bijectif (mais $A^{-1}$ n'est plus continu et les $A_{h}^{-1}$ ne sont plus stables) si $l^{\prime}$ on suppose seulement que $s(u, v)>0 \quad\left(\right.$ cf. $\left.n^{\circ} 3-5\right)$.

Nous allons maintenant donner deux exemples de classes d'opérateurs A vérifiant les conditions de stabilité $s_{1}$ et $s_{2}$.

EXEMPLE 3-1. OPERATEURS LINEAIRES COERCIFS.

Supposons que les formes $a_{h}\left(u_{h}, v_{h}\right)$ et $a(u, v)$ soient sesquilinéaires et globalement coercives :

(3-11) Il existe $c>0$ tel que $\left|a_{h}\left(v_{h}, v_{h}\right)\right| \geqslant c|| v_{h} \|_{h}^{2}$ pour tout $v_{h} \varepsilon v_{h}$. On sait alors (cf. Appendice) que les opérateurs $A_{h}$ sont des isomorphismes de $V_{h}$ sur $V_{h}^{\prime}$. Il est clair, d'autre part, que les conditions de stabilité $s_{1}$ et $\mathrm{S}_{2}$ sont vérifiées puisque :

$$
\left|s_{h}\left(u_{h}, v_{h}\right)\right|=\left|a_{h}\left(u_{h}-v_{h}, u_{h}-v_{h}\right)\right| \geqslant c|| u_{h}-v_{h}||_{h}^{2}
$$

On en déduit le :

COROLLAIRE 3-1.

Si les formes sesquilinéaires $a_{h}\left(u_{h}, v_{h}\right)$ sont globalement coercives et consistantes à une forme sesquilinéaire $a(u, v)$ coercive, les solutions $u_{h}$ convergent discrètement vers la solution u si $\varepsilon\left(f, f_{h}\right)$ tend vers 0 . Les erreurs discrètes vérifient l'inégalité :

$$
\left\|u_{h}-r_{h} u\right\|_{h} \leqslant c^{-1}\left(\psi_{u}(h)+\varepsilon\left(f, f_{h}\right)\right)
$$

Sous des hypothèses légèrement différentes, le corollaire 3-1 a été démontré dans J. CEA $[1]$. 
Supposons que $\mathrm{V}$ soit un espace de Hilbert contenu et dense dans un espace de Hilbert $\mathrm{H}$, et soit $\mathrm{A}=\{\mathrm{V}, \mathrm{H}, \mathrm{a}(\mathrm{u}, \mathrm{v})\}$ (cf. Appendice) 1 'isomorphisme du domaine $D(A)$, muni de la norme du graphe $[u]=\left(|u|^{2}+|A u|^{2}\right)^{1 / 2}$, sur $\mathrm{H}$. Nous allons approcher discrètement $u=A^{-1} f$ dans $D(A)$ lorsque $f$ appartient à $\mathrm{H}$.

Nous supposerons pour cela que les espaces $V_{h}$ sont de dimension finie.

On considère les normes suivantes sur $v_{h}$.

$$
\text { (3-13) }\left|u_{h}\right|_{h}=\left(u_{h}, u_{h}\right)_{h}^{1 / 2} ;\left[u_{h}\right]_{h}=\left(\left|u_{h}\right|_{h}^{2}+\left|A_{h} u_{h}\right|_{h}^{2}\right)^{1 / 2}
$$

La norme $\left[u_{h}\right]_{h}$ est la norme du graphe de l'opérateur $A_{h}$.

THEOREME $3-2$.

Supposons que les jormes $a_{h}\left(u_{h} ; v_{h}\right)$ et $a(u, v)$ soient globalement coercives et que

$$
\left\{\begin{array}{l}
\text { i) }\left|u_{h}\right|_{h} \leqslant k|| u_{h}||_{h} ; k \text { indēpendante de } h . \\
\text { ii) }\left\{\begin{array}{l}
\hat{\psi}_{u}(h)=\sup _{v_{h}}\left|v_{h}\right|_{h}^{-1}\left|a\left(u, p_{h} v_{h}\right)-a_{h}\left(r_{h} u, v_{h}\right)\right| \\
\text { tend vers } 0 \text { avec } h \text { pour tout } u \in D(A)
\end{array}\right. \\
\text { iii) }\left\{\begin{array}{l}
\text { Si } \mathrm{f} \varepsilon \mathrm{H}, \hat{\varepsilon}\left(\mathrm{f}, \mathrm{f}_{\mathrm{h}}\right)=\sup \left|\mathrm{v}_{\mathrm{h}}\right|_{\mathrm{h}}^{-1}\left|\left(\mathrm{f}, \mathrm{p}_{\mathrm{h}} \mathrm{v}_{\mathrm{h}}\right)-\left(\mathrm{f}_{\mathrm{h}}, \mathrm{v}_{\mathrm{h}}\right)_{h}\right| \\
\text { tend vers } 0 \text { avec } \mathrm{h}
\end{array}\right.
\end{array}\right.
$$

les solutions $u_{h}$ du problème $P_{h}$ convergent discrètement vers $u$ pour la norme $\left[\mathrm{u}_{\mathrm{h}}\right]_{\mathrm{h}}$ :

(3-15) $\left[u_{h}-r_{h} u_{h}\right]_{h} \leqslant k_{1}\left(\hat{\psi}_{u}(h)+\hat{\varepsilon}\left(f, f_{h}\right)\right.$ tend vers 0 avec $h$ :

Remarquons tout d'abord que sous les hypothèses du théorème 2 du $\$ 3$, les inégalités suivantes sont satisfaites :

$(3-16)\left\{\begin{array}{l}\text { i) } k^{-1}|| u_{h}||_{* h} \leqslant\left|u_{h}\right|_{h} \leqslant\left. k|| u_{h}\right|_{h} \leqslant k_{1}\left[u_{h}\right]_{h} \\ \text { ii) } \psi_{u}(h) \leqslant k \hat{\psi}_{u}(h) ; \varepsilon\left(f, f_{h}\right) \leqslant k \hat{\varepsilon}\left(f, f_{h}\right)\end{array}\right.$

La convergence discrète dans $\mathrm{D}(\mathrm{A})$ entraîne alors la convergence discrète dans $\mathrm{V}$.

Tout d'abord, il résulte de (3-16) et du corollaire 3-1 que $u_{h}$ converge discrètement vers $u$ dans $H$ puisque :

$$
\left|u_{h}-r_{h} u\right|_{h} \leqslant k^{2} c^{-1}\left(\hat{\psi}_{u}(h)+\hat{\varepsilon}\left(f, f_{h}\right)\right)
$$


Montrons ensuite que :

$\left|A_{h}\left(u_{h}-r_{h} u\right)\right|_{h} \leqslant \hat{\psi}_{u}(h)+\hat{\varepsilon}\left(f, f_{h}\right)$

En effet :

$$
\begin{aligned}
& \left|A_{h}\left(u_{h}-r_{h} u\right)\right|_{h}^{2}=a_{h}\left(u_{h}-r_{h} u, A_{h}\left(u_{h}-r_{h} u\right)\right)= \\
& \quad=\left(f_{h}, A_{h}\left(u_{h}-r_{h} u\right)\right)_{h}-\left(f, p_{h} A_{h}\left(u_{h}-r_{h} u\right)\right)+ \\
& \quad+a\left(u, p_{h} A_{h}\left(u_{h}-r_{h} u\right)-a_{h}\left(r_{h} u, A_{h}\left(u_{h}-r_{h} u\right)\right)\right. \\
& \quad \leqslant\left(\hat{\varepsilon}\left(f, f_{h}\right)+\hat{\psi}_{u}(h)\right)\left|A_{h}\left(u_{h}-r_{h} u\right)\right|_{h} \\
& \text { Si } k_{1}=\sqrt{k^{2} c^{-1}+1} \text {, on en déduit l'inégalité (3-15). }
\end{aligned}
$$

REMARQUE 3-2.

En fait, le théorème 3-2 est un corollaire du théorème 2-1 du $\$ 2 n^{\circ} 2-4$, puisque (3-14) ii) exprime la consistance des opérateurs $A_{h}$ à $A$ et puisque les opérateurs $A_{h}^{-1}$ sont stables au sens suivant :

$$
\left[A_{h}^{-1} f_{h}\right]_{h} \leqslant k_{2}\left|f_{h}\right|_{h} \quad ; \quad k_{2}=\sqrt{1+k^{2}}
$$

D'après le théorème $1-1$ du $\$ 1$, la convergence discrète dans $D(A)$ entraîne la convergence forte dans $D(A)$ si :

(3-17) $\left\{\begin{array}{l}\text { i) } p_{h} v_{h} \subset D(A) ;\left|p_{h} u_{h}\right| \leqslant\left|u_{h}\right|_{h} ;\left|A p_{h} u_{h}\right| \leqslant\left|A_{h} u_{h}\right|_{h} \\ \text { ii) } \lim _{h=0}|| u-p_{h} r_{h} u||_{D(A)}=0 \text { pour tout u de } D(A) .\end{array}\right.$

REMARQUE 3-3.

Puisque les espaces $V_{h}$ sont de dimension finie, il existe une constante $S(h)$ telle que :

(3-18) $\quad|| u_{h} \|_{h} \leqslant s(h)\left|u_{h}\right|_{h} \quad, \lim _{h=0} s(h)=+\infty$

Supposons que les opérateurs $A_{h}$ soient stables, c'est-à-dire que :

(3-19) $\left|a_{h}\left(u_{h}, v_{h}\right)\right| \leqslant M|| u_{h}||_{h}|| v_{h} \|_{h} ; M$ indépendante de $h$.

On en déduit les inégalités :

$(3-20)\left\{\begin{array}{l}\text { i) }\left[u_{h}\right]_{h} \leqslant\left(1+M^{2}\right)^{1 / 2} s(h)|| u_{h}||_{h} ; s(h)^{-1}|| u_{h}||_{h} \leqslant\left|u_{h}\right|_{h} \leqslant s(h)|| u_{h}||_{\star h} \\ \text { ii) } \hat{\psi}_{u}(h) \leqslant s(h) \psi_{u}(h) ; \hat{\varepsilon}\left(f, f_{h}\right) \leqslant s(h) \varepsilon\left(f, f_{h}\right)\end{array}\right.$ 
EXEMPLE 3-2.

Considérons des formes $b_{h}\left(u_{h} ; v_{h}, w_{h}\right)$ sur $v_{h}$, linéaires et continues en $v_{h}$ et $w_{h}$; supposons qu'il existe $c>0, L>0$ et $\rho>0$ tels que :

$(3-21)\left\{\begin{array}{l}\text { 1) } b_{h}\left(u_{h} ; v_{h}, v_{h}\right) \geqslant c|| v_{h} \|_{h}^{2} \text { si }\left\|u_{h}\right\|_{h} \leqslant \rho \\ \text { ii) }\left|b_{h}\left(u_{h}^{1} ; v_{h}, w_{h}\right)-b_{h}\left(u_{h}^{2}, v_{h}, w_{h}\right)\right| \leqslant L\left\|_{h}^{1}-u_{h}^{2}||_{h}\right\| v_{h}\left\|_{h}\right\| w_{h} \|_{h}\end{array}\right.$

et consídérons la forme $a_{h}\left(u_{h} ; v_{h}\right)$ suivante :

(3-22)

$a_{h}\left(u_{h} ; v_{h}\right)=b_{h}\left(u_{h} ; u_{h}, v_{h}\right)$

Si $\rho<\mathrm{CL}^{-1}$, les conditions $S_{1}$ et $S_{2}$ de stabilité sont vérifiées sur les boules $B_{h}^{\rho}$ de rayon $\rho$ puisque, d'après (3-21):

$$
(c-L \rho)\left\|u_{h}-v_{h}\right\|_{h}^{2} \leqslant s_{h}\left(u_{h}, v_{h}\right)
$$

On déduit alors du théorème 3-1 le :

COROLLAIRE 3-2.

Supposons que $\left\|\mathrm{f}_{\mathrm{h}}\right\|_{* \mathrm{~h}} \leqslant \mathrm{R}<\mathrm{c}^{2} \mathrm{~L}^{-1}$, que $\left\|\mathrm{r}_{\mathrm{h}} \mathrm{u}\right\|_{\mathrm{h}} \leqslant\|\mathrm{u}\|$ et que les formes $a(u ; v)$ et $a_{h}\left(u_{h} ; v_{h}\right)$ vérifient (3-21) et (3-22). Il existe alors des solutions uniques $u$ et $u_{h}$ des problèmes $P$ et $P_{h}$ vérifiant:

$$
\left\|u_{h}-r_{h} u\right\|_{h} \leqslant(c-L \rho)^{-1}\left(\psi_{u}(h)+\varepsilon\left(f, f_{h}\right)\right)
$$

Remarquons tout d'abord que si une solution $u_{h}$ du problème $P_{h}$ existe et que si $\left\|f_{h}\right\|_{\star h} \leqslant R$, alors $\left\|u_{h}\right\|_{h} \leqslant \rho=c^{-1} R$ puisque

$$
c|| u_{h}\left\|_{h}^{2} \leqslant b_{h}\left(u_{h} ; u_{h} ; u_{h}\right) \leqslant\left(f_{h}, u_{h}\right) \leqslant R\right\| u_{h} \|_{h}
$$

Il en résulte donc que si les solutions $u$ et $u_{h}$ existent, et si $\rho=c^{-1} \mathrm{R}<\mathrm{cL}^{-1}$, le corollaire 3-2 résulte du théorème 1 du $\$ 3$ et de (3-23). L'existence des solutions des problèmes $P$ et $P_{h}$ a été démontrée par J. CEA [3]: La méthode itérative :

$$
b_{h}\left(u_{h}^{n-1} ; u_{h}^{n}, v_{h}\right)=\left(f_{h}, v_{h}\right)_{h} \text { pour tout } v_{h} \varepsilon v_{h} ;\left\|u_{h}^{0}\right\| \leqslant \rho
$$

qui définit $u_{h}^{n}$ à partir de $u_{h}^{n-1}$ comme solution d'un problème linéaire est convergente. Ce résultat est un cas particulier du théorème 4-2 du $\$ 4$ que nous démontrerons. 
3-3. APPROXIMATION PAR LES RESTRICTIONS D'UN OPERATEUR. - ETUDE DE L'ERREUR.

Nous allons étudier 1'approximation d'un opérateur A par ses restrictions et dégager un certain nombre de résultats supplémentaires que $l^{\prime}$ on obtient dans ce cas concernant en particulier le comportement de l'erreur.

Nous supposerons que : Nous prendrons alors :

(3-27) $\left\{\begin{aligned} \text { i) } & \left\|u_{h}\right\|_{h}=\left\|p_{h} u_{h}\right\| \\ \text { ii) } & \left(f_{h}, v_{h}\right)_{h}=\left(f, p_{h} v_{h}\right) \quad\left(\text { i.e. : } f_{h}=r_{h}^{*} f \text { ou encore } \varepsilon\left(f, f_{h}\right)=0\right) \\ \text { iii) } & a_{h}\left(u_{h} ; v_{h}\right)=a\left(p_{h} u_{h} ; p_{h} v_{h}\right)\end{aligned}\right.$

Remarquons que sous ces hypothèses, la convergence discrète dans $V$ entrafne la convergence forte (théorème 1-1 du $\$ 1$ ) et que la forme $a_{h}\left(u_{h}, v_{h}\right)$ définie par (3-27) iii) est associée à la restriction $r_{h}^{*} A p_{h}$ de l'opérateur A (d'après (3-3) et (3-5) ). D'après (3-26), on sait que ces restrictions $A_{h}=r_{h}^{*} A p_{h}$ sont consistantes à A .

Si la forme a(u; v) vérifie l'une des conditions $s_{1}$ ou $s_{2}$, les formes $a_{h}\left(u_{h} ; v_{h}\right)$ les vérifient également, si bien que les opérateurs $A_{h}^{-1}$ sont stables (lorsqu'on les suppose surjectifs). On déduit alors du théorème $3-1$ que les hypothèses (3-26) et (3-27) impliquent la convergence forte des solutions $u_{h}$ des problèmes $P_{h}$ (lorsqu'elles existent) vers la solution u du problème $P$.

Il en résulte donc que la donnée d'approximations $A_{h}(V)$ vérifiant (3-26) permet de construire des problèmes approchés dans des espaces de dimension finie, dont les solutions sont convergentes.

Nous allons étudier l'évaluation du comportement de l'erreur entre les solutions $u$ et $u_{h}$ pour certaines classes d'opérateurs.

EXEMPLE 3-3. CAS DES OPERATEURS LINEAIRES COERCIFS.

Supposons que $\mathrm{V}$ soit un sous-espace dense d'un espace de Hilbert $\mathrm{H}$ et considérons le triplet $A=\{\mathrm{V}, \mathrm{H}, \mathrm{a}(\mathrm{u}, \mathrm{v})\}$ (cf. Appendice).

Si les restrictions $r_{h}$ sont linéaires, nous poserons :

(3-28) $r(h)=\sup _{u \in D(A)}\|u\|_{D(A)}^{-1}\left\|u-p_{h} r_{h} u\right\| ; r_{*}(h)=\sup _{u \in D\left(A^{*}\right)}\|u\|_{D\left(A^{*}\right)}^{* 1}\left\|u-p_{h} r_{h} u\right\|$ 
Nous allons étudier le comportement de l'erreur $u-p_{h} u_{h}$ dans les espaces $V$, $H$ et dans les espaces d'interpolation $S(\theta ; V, H)$ entre $V$ et $H$ (cf. Appendice)

THEOREME 3-3.

Si la forme $a(u, v)$ est sesquilinéaire, coercive et continue et si les hypothèses (3-26) et (3-27) sont satisjaites, il existe une constante $\mathrm{H}_{1}$ indēpendante de $\mathrm{h}$ telle que

(3-29) $\left\|u-p_{h} u_{h}\right\|_{S(\theta ; v, H)} \leqslant M_{1} \gamma_{*}(h)^{\theta}\left\|u-p_{h} u_{h}\right\| ; 0 \leqslant \theta \leqslant 1$

Si de plus $\mathrm{f} \varepsilon \mathrm{H}$, alors $\mathrm{u}$ appartient $\bar{a} \mathrm{D}(\mathrm{A})$ et

i) $\left\|u-p_{h} u_{h}\right\| \leqslant c^{-1} M \gamma(h)\|u\|_{D(A)}$

ii) $\left\|u-p_{h} u_{h}\right\|_{S(\theta ; v, H)} \leqslant M_{2} r(h) \gamma_{*}(h)^{\theta}$

Posons pour simplifier $\varepsilon_{h}=u-p_{h} u_{h}, T_{h}=1-p_{h} r_{h}, T_{h}^{*}=1-p_{h}^{*} r_{h}^{*}$

On remarque alors que :

$$
a\left(\varepsilon_{h}, v\right)=a\left(\varepsilon_{h}, T_{h} v\right) \text { pour tout } v \varepsilon D\left(A^{*}\right)
$$

Donc les erreurs $\varepsilon_{h}=u-p_{h} v_{h}$ vérifient :

$$
\varepsilon_{h}=A^{-1} T_{h}^{*} A \varepsilon_{h}
$$

Mais $A$ est un isomorphisme de $V$ sur $V^{\prime}$, de $H$ sur $D\left(A^{*}\right)^{\prime}$ et par suite, de $S(\theta ; V, H)$ sur $S\left(\theta ; V^{\prime}, D\left(A^{*}\right)^{\prime}\right)$. $D^{\prime}$ autre part, $T_{h}^{*}$ est un opérateur de $V^{\prime}$ dans $V^{\prime}$ avec une norme égale à 1 , de $V^{\prime}$ dans $D\left(A^{*}\right)^{\prime}$ avec une norme égale à $\gamma_{*}(h)$ d'après (3-28), donc de $V^{\prime}$ dans $s\left(\theta ; V^{\prime}, D\left(A^{*}\right)^{\prime}\right)$ avec une norme égale à $\gamma_{*}(h)^{\theta}$.

Donc $A^{-1} T_{h}^{*} A$ est un opérateur de $V$ dans $S(\theta ; V, H)$ avec une norme égale à $M_{1} \gamma_{*}(h)^{\theta}$, d'où (3-29).

Nous sommes donc ramenés à étudier la norme de l'erreur $u-p_{h} u_{h}$ dans l'espace $v$. Mais puisque

$$
a\left(u-p_{h} u_{h}, u-p_{h} u_{h}\right)=a\left(u-p_{h} u_{h}, u-p_{h} r_{h} u\right)
$$

on en déduit que :

(3-31) ||$u-p_{h} u_{h}\left\|\leqslant c^{-1} M|| u-p_{h} r_{h} u\right\|$ 
Si $f \in H$, on sait que $u$ appartient à $D(A)$, l'inégalité (3-30) 1) résulte alors de (3-31) et de (3-28) et l'inégalité (3-30) i1) résulte de (3-29) et de $(3-30)$ i) .

REMARQUE 3-4.

L'inégalité (3-31) est vraie, quelle que soit la restriction $r_{h}$, linéaire ou non. On peut prendre en particulier pour restriction $r_{h}$ la restriction de Tchebychev (cf. $\$ 1, n^{\circ} 1-9$ ) (proposition 1-6) qui minimise le second membre de l'inégalité (3-31) : on en déduit que l'erreur $\left\|u-p_{h} u_{h}\right\|$ est de l'ardre de la meilleure approximation de la solution u par les éléments de $\mathrm{p}_{\mathrm{h}} \mathrm{v}_{\mathrm{h}}$.

Dans le théorème 2 du $\$ 3$, pour $\theta=1$, on obtient

(3-32) $\quad\left|u-p_{h} u_{h}\right| \leqslant M_{1} \gamma_{*}(h)|| u-p_{h} u_{h}|| \leqslant M_{3} \gamma(h) \gamma_{*}(h)$

EXEMPLE 3-4 (cf. Exemple 3-2)

Supposons que $a(u ; v)=b(u ; u, v)$ où $1 a$ forme $b(u ; v, w)$ est linéaire et continue en $v$ et $w$ et où :

1) $\mathrm{b}(\mathrm{u} ; \mathrm{v}, \mathrm{v}) \geqslant \mathrm{c}\|\mathrm{v}\|^{2}$ si $\|\mathrm{u}\|<\mathrm{p}$

ii) $\left|b\left(u^{1} ; v, w\right)-b\left(u^{2} ; v, w\right)\right| \leqslant L|| u^{1}-u^{2} \||| v|||| w||$

(iii) $|b(u ; v, w)| \leqslant M(u)|| v|||| w||$

Considérons alors l'équation :

(3-34) $a_{h}\left(u_{h} ; v_{h}\right)=b\left(p_{h} u_{h} ; p_{h} u_{h} ; p_{h} v_{h}\right)=\left(f, p_{h} v_{h}\right)$ pour tout $v_{h}$ de $v_{h}$.

PROPOSITION 3-1.

Si $\rho<c L^{-1}$, les erreurs $u-p_{h} u_{h}$ entre les solutions $u$ et $u_{h}$ vérifient l'inégalité :

(3-35) $\left\|u-p_{h} u_{h}\right\| \leqslant\left(c-L_{\rho}\right)^{-1}\left(M(u)+L_{\rho}\right)\left\|u-p_{h} r_{h} u\right\|$

On remarque en effet, en utilisant les équations que :

$b\left(u ; u-p_{h} u_{h}, u-p_{h} u_{h}\right)=b\left(u ; u ; u-p_{h} r_{h} u\right)-b\left(p_{h} u_{h} ; p_{h} u_{h}, u-p_{h} r_{h} u\right)$

$+b\left(p_{h} u_{h} ; p_{h} u_{h}, u-p_{h} u_{h}\right)-b\left(u ; p_{h} u_{h}, u-p_{h} u_{h}\right)$.

En utilisant (3-33) et le fait que $\left\|p_{h} u_{h}\right\| \leqslant c^{-1} R=\rho$, on en déduit l'inégalité :

$\left(c-L_{\rho}\right)\left\|u-p_{h} u_{h}\right\|^{2} \leqslant(M(u)+L \rho)\left\|u-p_{h} u_{h}\right\|\left\|u-p_{h} r_{h} u\right\|$ 
EXEMPLE 3-5. : ETUDE DE L'ERREUR LORSQUE A EST NON LINEAIRE :

PROPOSITION 3-2.

Supposons que (3-27) soit vérifiée. Les erreurs $u-p_{h} u_{h}$ vérifient l'inégalité (3-36) $\quad s\left(u ; p_{h} u_{h}\right) \leqslant\left\|A(u)-A\left(p_{h} u_{h}\right)\right\|_{*}\left\|u-p_{h} r_{h} u\right\|$

Si A est un opérateur Höldêrien, on en déduit que :

$$
\frac{s\left(u ; p_{h} u_{h}\right)}{\| u-p_{h} u_{h}||^{\theta}} \leqslant M|| u-p_{h} r_{h} u \|
$$

EXEMPLE 3-6. RESTRICTIONS D'OPERATEURS DIFFERENTIABLES DE $v$ DANS $v^{\prime}$

Nous allons montrer que les restrictions $r_{h}^{*} A p_{h}$ d'un opérateur différentiable A vérifient, sous certaines conditions, les hypothèses du théorème 2-2 du $\$ 2$ $n^{\circ} 2-7$.

Supposons que l'opérateur A soit différentiable sur une boule $U$ de rayon $\rho+\varepsilon$ de $V$.

PROPOSITION 3-3.

Supposons que l'opérateur A soit borné, que sa différentielle soit bornée sur $\mathrm{u}$ et que :

$$
\|v\|^{\lim } \quad \sup _{u}|| v\left\|^{-1} \mid\right\|_{0}(u ; v) \|_{*}=0
$$

Supposons qu'il existe $u_{0}$ de $\mathrm{u}$ tel que $\left\|\mathrm{r}_{\mathrm{h}} \mathrm{u}_{0}\right\| \leqslant \rho$ et tel que :

(DA $\left.\left(p_{h} x_{h} u_{0}\right) v, v\right) \geqslant c|| v \|^{2} ; c>0$ indépendante de $h$.

Supposons enfin que (3-26) et (3-27) aient lieu et que les restrictions $r_{h}$ soient stables. Il existe $r$ tel que les solutions $u$ et $u_{h}$ des équations :

$$
a(u ; v)=(f, v) ; a\left(p_{h} u_{h}, p_{h} v_{h}\right)=\left(f, p_{h} v_{h}\right)
$$

existent dans un voisinage de $u_{0}$ et de $r_{h} u_{0}$ si $\left\|f-f_{0}\right\|_{*}<r$. De plus, $\mathrm{P}_{\mathrm{h}} \mathrm{u}_{\mathrm{h}}$ converge vers $\mathrm{u}$ dans $\mathrm{V}$.

En effet, si 1 'opérateur $A$ est différentiable, sa restriction $r_{h}^{\star} A p_{h}$ est également différentiable et :

$r_{h}^{*} A\left(p_{h} u_{h}+p_{h} v_{h}\right)=r_{h}^{*} A\left(p_{h} u_{h}\right)+r_{h}^{*} D A\left(p_{h} u_{h}\right) p_{h} v_{h}+r_{h}^{*} \omega\left(p_{h} u_{h} ; p_{h} v_{h}\right)$ 
Puisque :

$\left(D A\left(p_{h} r_{h} u_{o}\right) p_{h} v_{h}, p_{h} v_{h}\right) \geqslant\left. c|| p_{h} v_{h}\right|^{2} ; c>0$.

les différentielles $D_{h}\left(r_{h} u_{o}\right)=r_{h}^{*} D A\left(p_{h} r_{h} u_{o}\right) p_{h}$ sont inversibles et leurs inverses sont stables.

L'opérateur A étant en particulier continu sur $U$, la consistance des restrictions $r_{h}^{*} A p_{h}$ à A est satisfaite. La proposition 3-3 est alors un corollaire du théorème $2-2$ du $n^{\circ} 2-7$ du $\$ 2$.

Si 1 'on prend $u_{0}=0$, il suffit de supposer que :

$$
(D A(0) v, v) \geqslant c|| v \|^{2} ; c>0 \text {. }
$$

3-4. APPROXIMATION PAR LES RESTRICTIONS PARTIELLES D'UN OPERATEUR A •

Lorsque 1'opérateur $A=\sum_{q, r} A_{q r}$ est la somme d'opérateurs $A_{q r}$, nous construirons des opérateurs $A_{q r}$ dans des approximations partielles de l'espace $V$. Nous n'utiliserons pas de prolongements de $v$ dans $v_{h}$, (nous $n$ 'aurons donc pas de notion de consistance), mais nous aurons un résultat de convergence sous des hypothèses moins fortes.

Supposons que 1'espace $V$ soit un sous-espace fermé de l'intersection d'une suite finie d'espaces $v_{r}$, que nous supposerons, pour simplifier, contenus et denses dans 1 'espace $v_{0}$.

Considérons des formes $\mathrm{a}(\mathrm{u} ; \mathrm{v}), \mathrm{sur} \mathrm{V} \times \mathrm{V}$ de la forme suivante :

$$
a(u ; v)=\sum_{q, r} a_{q r}(u ; v)
$$

où les formes $a_{q r}(u ; v)$ sont définies sur $v_{q} \times v_{r}$, linéaires et continues sur $\mathrm{v}_{\mathbf{r}}$ par rapport à $\mathbf{v}$.

Donnons-nous des approximations partielles $\left(\$ 1, n^{0} 1-4\right)$ des espaces $v_{r}$ dans des espaces de dimension finie $V_{h}$

$$
A_{h}\left(v_{r}\right)=\left(v_{r}, v_{h}, p_{h}^{r}, r_{h}\right)
$$

et nous supposerons que :

$(3-37)\left\{\begin{array}{l}\text { i) }\left\|u_{h}\right\|_{h}=\left(\sum_{r} \| p_{h}^{r} u_{h}||_{r}^{2}\right)^{1 / 2} \text { est une norme de } v_{h} \\ \text { ii) les approximations } A_{h}\left(v_{r}\right) \text { sont convergentes. }\end{array}\right.$

Si $f \varepsilon V^{\prime}, f \quad s^{\prime e ́ c r i t ~(d e ~ m a n i e ̀ r e ~ n o n ~ u n i q u e) ~: ~}$

$$
f=\sum_{r} f_{r} \quad ; \quad f_{r} \in V^{\prime}{ }_{r}
$$


Nous considérons le problème approché $P_{h}$ suivant :

$(3-38) a_{h}\left(u_{h} ; v_{h}\right)=\sum_{q, r} a_{q r}\left(p_{h}^{q} u_{h} ; p_{h}^{r} v_{h}\right)=\left(f_{h}, v_{h}\right)_{h}=\sum_{r}\left(f_{r}, p_{h}^{r} v_{h}\right)$

Nous dirons que la forme $a_{h}\left(u_{h}, v_{h}\right)$ est la restriction de $a(u ; v)$ dans les approximations partielles $A_{h}\left(V_{q}\right)$.

Nous allons démontrer le :

THEOREME 3-4.

Supposons que

i) Pour tout $\varepsilon>0$, il existe $n>0$ tel que

$$
\sum_{\mathbf{r}} \mid u_{\mathbf{r}}-\mathrm{v}_{\mathbf{r}} \|_{\mathbf{r}}^{2} \geqslant \varepsilon \text { entraine que }\left|\sum_{\mathrm{q}, \mathrm{r}} s_{\mathrm{qr}}\left(u_{\mathbf{r}}, \mathrm{v}_{\mathbf{r}}\right)\right| \geqslant n
$$

ii) Pour tout $\mathrm{K}$, il existe $\mathrm{R}$ tel que

$$
\left\{\begin{array}{l}
\sum_{r}|| u_{r} \|_{r}^{2} \geqslant R^{2} \text { entraine que } \sum_{q, r} a_{q r}\left(u_{q}, u_{r}\right) \geqslant \underset{r}{K}\left(\sum_{r}\left\|u_{r}\right\|_{r}^{2}\right)^{1 / 2} \\
\text { iii) } \sup _{\text {iv) } a_{q r}(u, v)}\left|a_{q r}(u, v)\right| \leqslant c|| v \|_{r} \leqslant \text { est continue sur } v_{q} \text { par rapport à } u .
\end{array}\right.
$$

Si l'hypothèse (3-37) est vērifiēe, les salutions $u_{h}$ du problème $P_{h}$ existent sont uniques, et convergent vers la solution $u$ du problème $P$ au sens suivant : (3-40) $\quad \sum_{r}\left\|u-p_{h}^{r} u_{h}\right\|_{r}^{2}$ tend vers 0 avec $h$.

REMARQUE 3-5.

On peut, dans le théorème $4 \mathrm{du} \$ 3$, affaiblir 1 'hypothèse (3-39) iv) et renforcer 1 'hypothèse (3-39) 1 ) en les remplaçant respectivement par

$$
\begin{aligned}
& (3-39)^{\prime} 1 \text { ) : Pour tout } \varepsilon>0 \text {, il existe } n>0 \text { tel que }\left\{\left\|u_{r}-v_{r}\right\|_{r}^{2} \geqslant \varepsilon\right. \\
& \text { entraine } \sum_{q, r} s_{q r}\left(u_{r}, v_{r}\right) \geqslant n \\
& \text { (3-39)' iv) } \lim _{\lambda \rightarrow 0} a_{q r}\left(u-\lambda u_{0}, v\right)=a_{q r}(u, v) \text { pour tout } v \text { fixé. }
\end{aligned}
$$

On verra dans la démonstration que $l^{\prime}$ hypothèse $(3-39)^{\prime}$ lv) sert à montrer que la limite $u_{*}$ d'une suite est solution de l'équation. L'hypothèse (3-39)' 1 ) impliquant que les opérateurs sont monotones, le procédé de MINTY (cf. $n^{\circ} 3-5$ ) permet d'aboutir au même résultat sous 1 'hypothèse (3-39)' iv). 
Démonstration du théorème $4 d u \$ 3$.

Remarquons tout d'abord que les opérateurs $A_{h}$ vérifient la condition de stabilité $s_{2}$ d'après $(3-39)$ i) et ii). D'après la remarque $3-1$, les solutions $u_{h} e-$ xistent et sont uniques. De plus, de (3-39) ii) résulte que :

$$
\left\|u_{h}\right\|_{h}^{2}=\sum_{r}\left\|p_{h}^{r} u_{h}\right\|_{r}^{2} \leqslant \text { cste. }
$$

En effet, si on fait $v_{h}=u_{h}$ dans (3-38), nous avons :

$$
\frac{a_{h}\left(u_{h} ; u_{h}\right)}{\left\|u_{h}\right\|_{h}} \leqslant\left(\left[\left\|f_{r}\right\|_{* r}^{2}\right)^{1 / 2}\right.
$$

Si $\left\|u_{h}\right\|_{h}$ tendait vers l'infini, 11 en serait de même de $\left\|u_{h}\right\|_{h}^{-1} a_{h}\left(u_{h}, u_{h}\right)$ d'après (3-39) ii), ce qui contredit l'inégalité précédente.

On peut donc extraire de $h$ une sous-suite (encore notée $h$ ) de $h$ telle que (par définition des approximations partielles)

$$
\underset{h=0}{\lim } p_{h}^{r} u_{h}=u_{*} \text { dans } v_{r} \text { faible, pour tout } r .
$$

En fait, on déduit de (3-30) i) et iii) que $p_{h}^{r} u_{h}$ converge fortement vers $u_{*}$ dans $V_{r}$ puisque :

$$
\sum_{q, r} s_{q r}\left(u_{*}, p_{h}^{r} u_{h}\right)=\sum_{q, r} a_{q r}\left(u_{*}, u_{*}-p_{h}^{r} u_{h}\right)+\sum_{r}\left(f_{r}, p_{h}^{r} u_{h}-p_{h}^{r} r_{h} u_{*}\right)+
$$

$+\sum_{q, r} a_{q r}\left(p_{h}^{q} u_{h}, p_{h}^{r} r_{h} u_{*}-u_{*}\right)$

Donc, pour tout $\varepsilon>0$, 11 existe $n$ et $h$ assez petits tels que :

$$
\left|\sum_{q, r} s_{q r}\left(u_{\star}, p_{h}^{r} u_{h}\right)\right|<n
$$

ce qui entraine que $\sum_{r}\left\|u_{*}-p_{h}^{r} u_{h}\right\|_{r}^{2} \leqslant \varepsilon$

On déduit alors de (3-39) iv) que $u_{x}$ est solution du problème $P$ en prenant $v_{h}=r_{h} v$ dans (3-38). Cette solution étant unique, on en déduit (3-40).

Les hypothèses (3-39) sont vérifiées en particulier si (cf. exemples 3-1 et 3-3, 3-2 et 3-4)

a) - Les formes $a_{q r}(u, v)$ sont sesquilinéaires continues sur $v_{q} \times v_{r}$ et vérifient :

(3-41) $\quad \sum_{q, r} a_{q r}\left(u_{q}, v_{r}\right) \geqslant c \sum_{q}\left\|u_{q}\right\|_{q}^{2}$. 
b) $-a(u ; v)=b(u ; u, v)=\sum_{q, r, s} b_{q r s}(u ; u, v)$

où les formes $b_{q r s}$ sont définies sur $v_{q} \times v_{r} \times v_{s}$ et vérifient :

$(3-42)\left\{\begin{array}{l}\text { i) } \underset{r, s}{\sum_{q q s}} b_{q u}\left(u ; v_{r}, v_{s}\right) \geqslant c \sum_{r}\left\|v_{r}\right\|_{r}^{2} \\ \text { ii) }\left|b_{q r s}\left(u^{2} ; v_{r}, v_{s}\right)-b_{q r s}\left(u^{2}, v_{r}, v_{s}\right)\right| \leqslant\left.\left. L\left\|u^{1}-u^{2}\right\|\right|_{q}\left\|v_{r}\right\|\right|_{r}\left\|v_{s}\right\|_{s}\end{array}\right.$

On prendra alors :

$$
a_{h}\left(u_{h}, v_{h}\right)=\sum_{q, r, s} b_{q r s}\left(p_{h}^{q} u_{h} ; p_{h}^{r} u_{h}, p_{h}^{s} v_{h}\right)
$$

et les conclusions du théorème auront lieu si :

$$
\|f\|_{\star} \leqslant R \leqslant c^{2} L^{-1} \text {. }
$$

Les applications des résultats du début de ce paragraphe sont exposées au $n^{\circ} 4-2$ du $\$ 4$ du chapitre II.

\section{3-5. APPROXIMATION FAIBLE DES OPERATEURS MONOTONES.}

Le théorème 1 du $\$ 3$ assure 1 'unicité des solutions du problème $P$ sous des hypothèses assez fortes pour entrafner en plus, la convergence forte des solutions des opérateurs approchés. Sous des hypothèses moins fortes, J. LERAY et J.L. LIONS [1] , F. BROWDER , G. MINTY , V. VISIK ont démontré un théorème d'existence. Nous allons obtenir dans ce cas la convergence faible des solutions des problèmes approchés : nous supposerons que ces opérateurs sont monotones, coercifs, bornés et continus des droites de $V$ dans $V^{\prime}$ faible. Nous utiliserons ce théorème au $n^{\circ} 4-3$ du $\$ 4$ du chapitre II.

THEOREME 3-5.

Sous les hypothèses suivantes:

(3-43) A est borné et $\lim _{\lambda=0} a(u-\lambda w ; v)=a(u ; v)$

$$
\begin{aligned}
& \text { i) } s_{h}\left(u_{h}, v_{h}\right) \geqslant 0 \quad(s(u, v) \geqslant 0) \quad \text { (monotonie) } \\
& \text { ii) } \quad 1 \text { im }\left\|u_{h}\right\|_{h}^{-1} \quad a_{h}\left(u_{h}, u_{h}\right)=\infty \quad \text { (coercivité) } \\
& \left\|u_{h}\right\|_{h}=\infty
\end{aligned}
$$

(3-45) $\left\{\begin{array}{l}\text { i) Les espaces } v_{h} \text { sont de dimension finie, les } p \text { ro } \\ \text { bles, les approximations } A_{h}(v) \text { convergentes. } \\ \text { ii) } 1 \text { im } \Psi_{u}(h)=0 ; \lim \varepsilon\left(f, f_{h}\right)=0\end{array}\right.$ 
On peut extraire de la suite $p_{h} u_{h}$ une sous-suite faiblement convergente vers ure solution $u_{*}$ du problème $P$.

REMARQUE 3-6.

Si l'on suppose que $s_{i a}\left(u_{h}, v_{h}\right)>0$ et $s(u, v)>0$. les solutions $u$ et $u_{h}$ des problèmes $P$ et $P_{h}$ sont uniques. Le théorème $3-5$ implique alors que $p_{h} u_{h}$ converge faiblement vers $u$.

DEMONSTRATION.

Tout d'abord, $\left\|p_{h} u_{h}\right\|$ est borné d'après (3-44) ii): si $\left\|u_{h}\right\|_{h}$ tendait vers l'infini, on aurait :

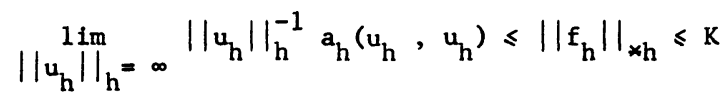

ce qui est contradictoire. Désignons par $u_{\star}$ la limite faible d'une sous-suite extraite encore notée $p_{h} u_{h}$ et montrons que $u_{*}$ est solution du problème $P$.

Nous avons, si $v$ est un élément arbitraire de $\mathrm{V}$ :

$0 \leqslant s_{h}\left(u_{h}, r_{h} v\right)=\left(f, p_{h}\left(u_{h}-r_{h} v\right)\right)-a\left(v ; p_{h} u_{h}-p_{h} r_{h} v\right)+a\left(v ; p_{h}\left(u_{h}-r_{h} v\right)\right)$

$-a_{h}\left(r_{h} v, u_{h}-r_{h} v\right)+\left(f_{h}, u_{h}-r_{h} v\right)_{h}-\left(f, p_{h}\left(u_{h}-r_{h} v\right)\right)$

Nous pouvons donc passer à la limite où nous obtenons (d'après (3-45) i1)) :

$0 \leqslant \lim _{h=0} s_{h}\left(u_{h}, r_{h} v\right)=\left(f-A(v), u_{*}-v\right)$

Nous utilisons maintenant un procédé dû à G. MINTY :

Soit $\lambda>0$ et $w$ un élément arbitraire de $v$; posons $v=u_{x}-\lambda$ w. On déduit alors de 1 'inégalité précédente que :

$$
\left(f-A\left(u_{*}-\lambda w\right), w\right) \geqslant 0 \text { pour tout } w \text { fixé dans } v \text {. }
$$

Faisons tendre $\lambda$ vers 0 . D'après (3-43), nous obtenons :

$\left(f-A\left(u_{*}\right) ; w\right) \geqslant 0$ pour tout $w$ dans $V$.

Cela entraîne que $A\left(u_{*}\right)=f$, ce qui achève la démonstration du théorème.

APPROXIMATIONS PAR LES RESTRICTIONS PARTIELLES DE A (cf. n $^{\circ}$-4).

Supposons que les conditions (3-37) et (3-38) alent lieu et que

(3-46) i) $\|u\|_{q} \leqslant M\left(\sup _{q r}(u ; v)|\leqslant c| \mid v \|_{r}\right.$ 
$(3-46)\left\{\begin{array}{l}\text { ii) } \lim _{\lambda=0} a_{q r}(u-\lambda v ; w)=a_{q r}(u, w) \\ \text { iii) } \sum_{\|} \lim _{q} \|_{q}^{2}=\infty \\ \text { iv) } \sum_{q, r} s_{q r}\left(u_{q}, v_{r}\right) \geqslant 0\end{array}\right.$

PROPOSITION 3-4.

Sous les hypothèses (3-37), (3-38), et (3-46) on peut extraire des sous-suites de $p_{h}^{q} u_{h}$ convergeant jaiblement vers une solution $u_{*}$ dans $v_{q}$ pour tout $q$.

La démonstration est analogue à la précédente. On établit tout d'abord que $\left\|u_{h}\right\|_{h}^{2}=\sum_{q}\left\|p_{h}^{q} u_{h}\right\|_{q}^{2}$ est borné. Par définition, des approximations partielles $\left(\$ 1, n^{\circ} 1-4\right)$, il existe alors un élément $u_{*}$ de $v$ tel que $u_{*}=\lim _{h=0} p_{h}^{q} u_{h}$ dans $v_{q}$ faible pour tout $q$. On utilise enfín le procédé de Minty pour montrer que $u_{*}$ est solution du problème : $s i$

$0 \leqslant \sum_{q, r} a_{q r}\left(p_{h}^{q} u_{h} ; p_{h}^{r} u_{h}-v\right)-a_{q r}\left(v ; p_{h}^{r} u_{h}-v\right)$

Puisque $\sum_{q, r} a_{q r}\left(p_{h}^{q} u_{h} ; p_{h}^{r} u_{h}\right)=\sum_{r}\left(f_{r}, p_{h}^{r} u_{h}\right)$, on en déduit, par passage à

la limite, que

$$
\sum_{q, r}\left(f_{r}-A_{q r}(v), u_{*}-v\right) \geqslant 0 \text { pour tout } v \text { de } v \text {. }
$$

Il en résulte alors, comme précédemment, que

$$
\sum_{q, r} A_{q r}\left(u_{*}\right)=\sum_{r} f_{r}
$$

3-6. APPROXIMATION DES SOLUTIONS DEFINIES PAR LE LEMME DES PROJECTIONS.

Rappelons l'énoncé du lemrne des projections, qui est un critère permettant d'affirmer qu'un opérateur linéaire est surjectif. (cf. J.L. LIONS, [1], chap. 3).

Nous nous plaçons dans la situation suivante. On se donne

a) un espace de Hilbert $V$, muni du produit scalaire $\langle u, v\rangle$ et de la norme $\|u\|_{v}$

b) un espace prehilbertien $\Phi$, contenu dans $V$ avec une topologie plus fine, muni du produit scalaire $(((\phi, \psi)))$ : 


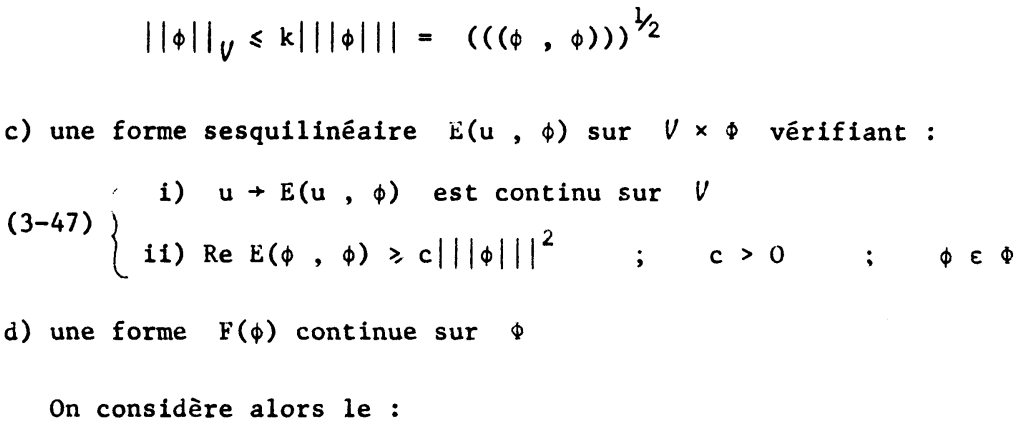

PROBLEME P. Chercher $u$ dans $V$ verrifiant : (3-48) $\quad E(u, \phi)=F(\phi)$ pour tout $\phi$ de $\phi$.

Le lemme des projections affirme alors qu'il existe au moins une solution $u$ du problème $P$ (cf. démonstration du théorème 3-6).

Donnons-nous des approximations $A_{h}(V)$ et $A_{h}(\Phi)$ des espaces $V$ et $\Phi$ :

$$
\left\{\begin{aligned}
& \text { i) } A_{h}(V)=\left(V, V_{h}, p_{h}, r_{h}\right) \\
& \text { ii) } A_{h}(\Phi)=\left(\Phi, \Phi_{h}, p_{h}, s_{h}\right) ; \Phi_{h} \subset V_{h} \\
& \text { iii) }\left\|\phi_{h}\right\|_{h} \leqslant k\|\| \phi_{h} \|\left.\right|_{h} \text { pour tout } \phi_{h} \text { de } \Phi_{h}
\end{aligned}\right.
$$

Donnons ensuite des formes $E_{h}\left(u_{h}, \phi_{h}\right)$ sur $V_{h} \times \Phi_{h}$ telles que

$$
\left\{\begin{array}{l}
\text { i) } u_{h} \rightarrow E_{h}\left(u_{h}, \phi_{h}\right) \text { est continue sur } v_{h} \\
\text { ii) } \operatorname{Re} E_{h}\left(\phi_{h}, \phi_{h}\right) \geqslant c\|\| \phi_{h} \|\left.\right|_{h} ; c>0 .
\end{array}\right.
$$

et des formes $F_{h}$ sur $\Phi_{h}$ telles que $\quad\left|F_{h}\left(\phi_{h}\right)\right| \leqslant c_{1} \mid\left\|\phi_{h}\right\| \|_{h}$

Considérons alors le :

PROBLEME $P_{h}$. Chercher $u_{h}$ dans $v_{h}$ vérifiant:

(3-51) $E_{h}\left(u_{h}, \phi_{h}\right)=F_{h}\left(\phi_{h}\right)$ pour tout $\phi_{h}$ de $\Phi_{h}$.

Il existe au moins une solution $u_{h}$ du problème $P_{h}$ • Remarquons aussi que les formes $E_{h}\left(u_{h}, \phi_{h}\right)=E\left(p_{h} u_{h}, p_{h} \phi_{h}\right)$ vérifient (3-49) si $\left\|\phi_{h}\right\|_{h}=\left\|p_{h} \phi_{h}\right\|$ et $\quad\left\|\phi_{h}\right\|\left\|_{h}=\right\| p_{h} \phi_{h}\|\|$. 
THEOREME 3-6.

Sous les hypothèses (3-47) à (3-50), il existe des solutions particulières du problème $P_{h}$ telles que :

(3-5z) $\quad|| P_{h} u_{h}\|\leqslant M\||| F_{h}\|\|_{h}$

(stabilitê)

si

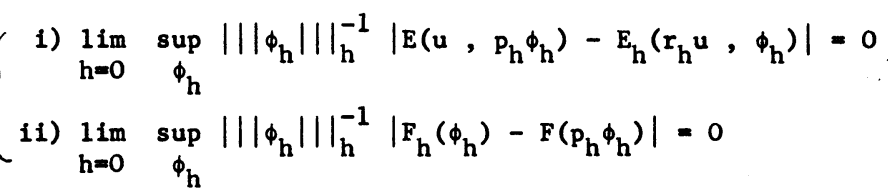

Les solutions $u_{h}$ convergent discrètement vers une solution $u$ du problème $P$. si

$$
\left\{\begin{aligned}
\text { i) } & \lim _{h=0} \sup _{v_{h}}\left\|u_{h}\right\|_{h}^{-1}\left|E\left(p_{h} u_{h}, \phi\right)-E_{h}\left(u_{h}, s_{h} \phi\right)\right|=0 \\
\text { ii) } & \lim _{h=0}\left|F(\phi)-F_{h}\left(s_{h} \phi\right)\right|=0 \\
\text { iii) } & \left\|p_{h} u_{h}\right\| \leqslant M_{1}\left\|u_{h}\right\|_{h} ;\left.\quad\left\||| F_{h}\right\|\right|_{h}<M_{2}
\end{aligned}\right.
$$

On peut extraire une sous-suite de $p_{h} u_{h}$ convergeant faiblement vers une solution u de E.

Nous allons tout d'abord établir (3-52). La forme $E_{h}\left(u_{h}, \phi_{h}\right)$ étant continue par rapport à $u_{h}$, elle définit un opérateur $A_{h}$ de $\Phi_{h}$ dans $V_{h}$ (identifié à son dual) par

$$
E_{h}\left(u_{h}, \phi_{h}\right)=\left\langle u_{h}, A_{h} \phi_{h}\right\rangle_{h} ; \quad u_{h} \varepsilon v_{h} \text {. }
$$

Posons $\Psi_{h}=A_{h} \Phi_{h}$.

On déduit de (3-50) ii) que $A_{h}$ est injective et que $A_{h}^{-1}$, définie de $\Psi_{h}$ sur $\Phi_{h}$, est continue : en effet, si $\psi_{h}=A_{h} \phi_{h}$,

$$
\begin{aligned}
c\|\| \phi_{h}\|\|_{h}^{2}=c\|\| A_{h}^{-1} \psi_{h}\|\|_{h}^{2} & \leqslant \operatorname{Re} E_{h}\left(\phi_{h}, \phi_{h}\right) \\
& =\operatorname{Re}<\phi_{h}, A_{h} \phi_{h}>_{h} \leqslant\left\|\phi_{h}\right\|\left\|_{h}\right\| \psi_{h} \|_{h}
\end{aligned}
$$

d'où l'on déduit que si. $A_{h} \phi_{h}=0, \phi_{h}=0$ et

(3-56) $\quad\left\|A_{h}^{-1} \phi_{h}\right\|_{h} \leqslant k c^{-1}\left\|\phi_{h}\right\|_{h}$

L'opérateur $A_{h}^{-1}$ se prolonge par continuité de l'adhérence $\bar{\psi}_{h}$ de $\Psi_{h}$ dans 
$V_{h}$. Puisque $V_{h}$ est un espace de Hilbert, 11 existe un projecteur orthogonal $\pi_{h}$ de $V_{h}$ sur $\bar{\Psi}_{h}$, de norme 1 .

On pose alors, si $\hat{\Phi}_{h}$ est le complété de $\Phi_{h}$ :

(3-57) $\quad T_{h}^{*}=A_{h}^{-1} \pi_{h} \quad ; \quad\left\|T_{h}^{*} v_{h}\right\|\left\|_{h} \leqslant k c^{-1}\right\| v_{h} \|_{h}$.

D'autre part, 1 'équation (3-51) s'écrit :

$<u_{h}, v_{h}>_{h}=F_{h}\left(A_{h}^{-1} v_{h}\right)$ pour tout $v_{h}$ de $\Psi_{h}$.

Donc toute solution de l'équation :

$(3-58)<u_{h}, v_{h}>_{h}=F_{h}\left(A_{h}^{-1} \pi_{h} v_{h}\right)=F_{h}\left(T_{h}^{*} v_{h}\right)$ pour tout $v_{h}$ de $v_{h}$ est en particulier solution de (3-51).

En désignant par $T_{h}$ l'adjoint de $T_{h}^{*}$, on en déduit que (3-59) $\quad u_{h}=T_{h} F_{h}$ dans. $V_{h}$

est une solution particulière du problème $P_{h}$. La norme de $T_{h}$ étant bornée, on en déduit $(3-52)$.

Considérons la solution $u=T F$ dans $V$ du problème $P$. Les erreurs discrètes $u_{h}-r_{h} u$ vérifient l'équation :

(3-60) $\quad E_{h}\left(u_{h}-r_{h} u, \phi_{h}\right)=G_{h}\left(\phi_{h}\right)$

où

$G_{h}\left(\phi_{h}\right)=F_{h}\left(\phi_{h}\right)-F\left(p_{h} \phi_{h}\right)+E\left(u, p_{h} \phi_{h}\right)-E_{h}\left(r_{h} u, \phi_{h}\right)$

Les hypothèses (3-53) entraînent que \|\|$G_{h}\|\|_{h}$ tend vers 0 , ce qui implique que :

$\left\|u_{h}-\left.r_{h} u\right|_{h}=\right\| T_{h} G_{h}\left\|_{h} \leqslant M\right\||| G_{h} \|\left.\right|_{h}$ tend vers 0 avec $h$.

Supposons (3-54). Puisque $\left\|\mid F_{h}\right\| \|_{h}$ est borné, $\left\|u_{h}\right\|_{h} 1$ 'est également, et par suite ||$p_{h} u_{h} \|_{h}$ est dans un borné de $V$. On peut extraire une sous-suite faiblement convergente vers $u_{*}$ dans $V$. Puisque :

$$
\begin{aligned}
& E\left(p_{h} u_{h}, \phi\right)=E\left(p_{h} u_{h}, \phi\right)-E_{h}\left(u_{h}, s_{h} \phi\right)+F_{h}\left(s_{h} \phi\right) \\
& \text { On en déduit que } E\left(u_{*}, \phi\right)=F(\phi) \text { pour tout } \phi \text { de } \phi .
\end{aligned}
$$

Nous donnerons des applications de ce numéro au $\mathrm{n}^{\circ}$ 5-3 du $\$ 5$ du chapitre II, pour construire des schémas à plusieurs niveaux des équations paraboliques.

\$4. APPROXIMATION DES SOLUTIONS DES INEQUATIONS VARIATIONNELLES.

On définit au $n^{\circ}$ 4-1 le problème et on établit au $n^{\circ} 4-2$ plusieurs résultats 
d'approximation.

On suppose connu le $\$ 3$ dans son ensemble. Les applications de ce numéro sont exposées au $n^{\circ}$ 4-4 du $\$ 4$ du Chapitre II .

4-1. POSITION DU PROBLEME.

Donnons-nous un ensemble convexe fermé $K$ de $V$ (réel) et une forme $a(u ; v)$ sur $\mathrm{V} \times \mathrm{V}$ linéaire et continue en $\mathrm{v}$. Si $f$ appartient à $\mathrm{V}^{\prime}$, on considère dans ce paragraphe le :

PROBLEME P •

Chercher $\mathrm{u}$ dans $\mathrm{K}$ solution de l'inéquation variationnelle (4-1) $\quad a(u ; u-v) \leqslant(f, u-v)$ pour tout $v$ de $\mathrm{K}$.

Si $\mathrm{K}$ est un cône convexe fermé, l'inéquation (4-1) est équivalente à : $\left\{\begin{array}{l}a(u ; v)=(A(u), v) \geqslant(f, v) \text { pour tout } v \text { de } K . \\ a(u ; u)=(A(u), u)=(f, u)\end{array}\right.$

Si $K$ est une variété linéaire affine de $V$, l'inéquation (4-1) est une équation. Si $K=V$, on retrouve les problèmes abordés au paragraphe précédent.

Si la forme $a(u, v)$ est bilinéaire, symétrique et coercive et $v$ un espace de Hilbert, la solution $u$ est la projection de $f$ sur le cône $K$ (lorsque $V$ est muni du produit scalaire $a(u, v)$ ) ou encore l'élément u qui minimise la fonctionnelle $a(v, v)-2(f, v)$ sur $K$.

Si la forme a(u; v) n'est pas symétrique, le théorème d'existence d'une solution $u$ est dû à G. STAMPACCHIA [1] [2]. Si la forme $a(u ; v)$ est monotone, coercive et bornée, le théorème d'existence a été démontré par P. HARTMAN et G. STAMPacchia [1] .

Donnons-nous maintenant des approximations $A_{h}(v)$ de $v$, une forme $a_{h}\left(u_{h} ; v_{h}\right)$ sur $v_{h}$, et un ensemble convexe fermé $k_{h}$ de $v_{h}$. On considère le:

PROBLEME $\mathrm{P}_{\mathrm{h}} \cdot$

Chercher $u_{h}$ dans $k_{h}$ solution de l'inéquation variationnelle: (4-3) $a_{h}\left(u_{h} ; u_{h}-v_{h}\right) \leqslant\left(f_{h}, u_{h}-v_{h}\right)_{h}$ pour tout $v_{h}$ de $k_{h}$. 
Rappelons que nous avons posé :

$$
\begin{aligned}
& \varepsilon\left(f, f_{h}\right)=\sup _{v_{h}}|| v_{h}||_{h}^{-1}\left|\left(f, p_{h} v_{h}\right)-\left(f_{h}, v_{h}\right)_{h}\right| \\
& \psi_{u}(h)=\sup _{v_{h}}|| v_{h}||_{h}^{-1}\left|a\left(u ; p_{h} v_{h}\right)-a_{h}\left(v_{h} u ; v_{h}\right)\right| \\
& s_{h}\left(u_{h}, v_{h}\right)=a_{h}\left(u_{h} ; u_{h}-v_{h}\right)-a_{h}\left(v_{h} ; u_{h}-v_{h}\right)
\end{aligned}
$$

4-2. THEOREMES DE CONVERGENCE.

THEOREME 4-1.

Supposons que les problèmes $P$ et $P_{h}$ admettent des solutions dans $\mathrm{K}^{\rho}=\mathrm{K} \cap \mathrm{B}(\rho)$ et $\mathrm{K}_{\mathrm{h}}^{\rho}=\mathrm{K}_{\mathrm{h}} \cap \mathrm{B}_{\mathrm{h}}(\rho)$ respectivement, où $\mathrm{B}_{\mathrm{h}}(\rho)$ est la boule de rayon $\rho>0$.

Supposons en outre que

(4-4) $\quad \mathrm{P}_{\mathrm{h}} \mathrm{K}_{\mathrm{h}} \subset \mathrm{K} \quad ; \quad \mathrm{r}_{\mathrm{h}} \mathrm{K}^{\rho} \subset \mathrm{K}_{\mathrm{h}}^{\rho}$

Les erreurs discrètes vērifient les inégalités

$s_{h}\left(u_{h}, r_{h} u\right) \leqslant\left(\psi_{u}(h)+\varepsilon\left(f, f_{h}\right)\right)\left\|u_{h}-r_{h} u\right\|_{h}+\|f-A(u)\|\left\|_{*}\right\| u-p_{h} r_{h} u \|$

Si les opérateurs $A_{h}$ vérifient la condition de stabilité $S_{2}{ }_{2}$

i) Pour tout $\varepsilon$, il existe $n$ tel que $\left\|u_{h}-v_{h}\right\|_{h} \geqslant \varepsilon$ entraine $s_{h}\left(u_{h}, v_{h}\right) \geqslant n$ Il existe $u_{0}$ dans $\mathrm{K}^{\rho}$ tel que :

(ii) $\lim _{\left\|u_{h}\right\|_{h} \rightarrow \infty} \frac{s_{h}\left(u_{h}, r_{h} u_{0}\right)}{\left\|u_{h}-r_{h} u_{0}\right\|}=\infty$ uniformément en $h$.

et $\mathrm{si}$

$$
\begin{aligned}
& \text { (4-6) } \lim _{h=0} \varepsilon\left(f, f_{h}\right)=0 ; \lim _{h=0} \psi_{u}(h)=0 \text { pour tout } u \text { de } k^{0} \\
& \text { (4-7) } \lim _{h=0}|| p_{h} r_{h} u-u||=0 ;\left.|| p_{h} u_{h}\left\|\leqslant M|| u_{h}\right\|\right|_{h}
\end{aligned}
$$

Les solutions $u_{h}$ du problème $P_{h}$ sont uniques et convergent vers la solution $u$ du problème $P$. 
DEMONSTRATION té :

Puisque d'après (4-4), $r_{h} u \varepsilon K_{h}^{\rho}$ et $p_{h} u_{h} \varepsilon K$, on déduit (4-5) de 1'inégali-

$$
\begin{aligned}
& s_{h}\left(u_{h}, r_{h} u\right) \leqslant a\left(u ; p_{h}\left(u_{h}-r_{h} u\right)\right)-a_{h}\left(r_{h} u ; u_{h}-r_{h} u\right) \\
& \quad+\left(f_{h}, u_{h}-r_{h} u\right)_{h}-\left(f, p_{h}\left(u_{h}-p_{h} r_{h} u\right)\right) \\
& \quad+\left(f, u-p_{h} r_{h} u\right)-a\left(u ; u-p_{h} r_{h} u\right)
\end{aligned}
$$

puisque, d'après (4-1) et (4-3), $a_{h}\left(u_{h} ; u_{h}-r_{h} u\right) \leqslant\left(f_{h}, u_{h}-r_{h} u\right)_{h}$ et $a\left(u ; u-p_{h} u_{h}\right)-\left(f, u-p_{h} u_{h}\right) \leqslant 0$

D'autre part, on déduit de la deuxième partie de la condition de stabilité $\mathrm{S}_{2}^{\prime}$ que

$$
|| u_{h}||_{h} \leqslant k
$$

puisque, si $\left\|u_{h}\right\|_{h}$ tendait vers l'infini, alors $s_{h}\left(u_{h}, r_{h} u_{o}\right)\left\|u_{h}-r_{h} u_{0}\right\|$ tendrait vers l'infini, ce qui contredit le fait que

$$
s_{h}\left(u_{h}, r_{h} u_{0}\right) \leqslant\left(f_{h}, u_{h}-r_{h} u_{0}\right) \leqslant\left\|f_{h}\right\|\left\|_{* h}|| u_{h}-r_{h} u_{0}\right\|
$$

Donc, en particulier $\left\|u_{h}-r_{h} u\right\|_{h}$ est borné. On en déduit la convergence discrète en utilisant la convergence discrète de $f_{h}$ vers $f$, la consistance des opérateurs $A_{h}$ à $A$, la convergence des approximations $A_{h}(V)$ et la première partie de la condition de stabilité $\mathrm{S}_{2}{ }_{2}$

De plus, les solutions $u_{h}$ sont uniques : sinon, si $\varepsilon=\left\|u_{h}-v_{h}\right\|_{h} \neq 0$ et si $u_{h}$ et $v_{h}$ vérifient $(4-3)$, on aurait, d'après (4-5):

$$
0=s_{h}\left(u_{h}, v_{h}\right) \geqslant n|| u_{h}-v_{h} \|_{h} ; n>0
$$

ce qui est contradictoire.

Si on suppose de plus que les prolongements sont stables, on obtient la convergence forte de $p_{h} u_{h}$ vers $u$ dans $V$, et par suite, 1'unicité de la solution du problème $\mathrm{P}$.

EXEMPLE 4-1. - INEQUATIONS A COEFFICIENTS LINEAIRES :

COROLLAIRE 4-1.

Si les formes $a_{h}\left(u_{h}, v_{h}\right)$ et $a(u, v)$ sont bilinéaires et globalement coercives, les solutions $u_{h}$ convergent discrètement vers $u$ sous les hypothèses (4-4), (4-6) et (4-7) et vérifient l'inégalité :

(4-8) ||$u_{h}-r_{h} u \|_{h}^{2} \leqslant c^{-1}\left[\left(\psi_{u}(h)+\varepsilon\left(f, f_{h}\right)\right)^{2} c^{-1}+2|| f-A(u) \|_{\star}|| u-p_{h} r_{h} u||\right]$ 
Si $a_{h}\left(u_{h}, v_{h}\right)=a\left(p_{h} u_{h}, p_{h} v_{h}\right)$ et $\left(f_{h}, v_{h}\right)_{h}=\left(f, p_{h} v_{h}\right)$, les erreurs $\mathrm{u}-\mathrm{p}_{\mathrm{h}} \mathrm{u}_{\mathrm{h}}$ obéissent à ia majoration :

(4-9) $\left\|u-p_{h} u_{h}\right\|^{2} \leqslant\left(\left\|f-A\left(p_{h} u_{h}\right)\right\|_{*}\left\|u-p_{h} r_{h} u\right\|\right)$

Considérons de plus le cas où $A_{h}$ est la somme des restrictions partielles d'un opérateur A (cf. $\left.\$ 3, \mathrm{n}^{\circ} 3-4\right)$. On suppose que :

$\left\{\begin{array}{l}a(u, v)=\sum_{q, r} a_{q r}(u, v) ;\left|a_{q r}(u, v)\right| \leqslant M|| u\left\|_{q}|| v\right\|_{r} \\ \sum a_{q r}\left(u_{q}, u_{r}\right) \geqslant c \sum_{r}|| u_{r} \|_{r}^{2} ; c>0 .\end{array}\right.$

On se donne des approximations $A_{h}\left(V_{r}\right)$ de $V_{r}$ dans des espaces de dimension finie $v_{h}$ telles que :

$$
\begin{aligned}
& \text { i) }\left\|u_{h}\right\|_{h}=\left(\sum_{r}\left\|p_{h}^{r} u_{h}\right\|_{r}^{2}\right)^{\frac{1}{2}} \text { est une norme de } v_{h} \\
& \text { ii) Si } u_{h} \varepsilon k_{h} \text { et si } p_{h}^{r} u_{h} \text { converge faiblement vers } u_{r} \text { dans } v_{r} \\
& \text { pour tout } r \text {, alors } u_{r}=u \text { appartient a } k \text {. } \\
& \text { iii) } \lim _{h=0}\left\|p_{h}^{r} r_{h} u-u\right\|_{r}=0 \text { pour tout u de } k \text { et pour tout } r \\
& \text { iv) } r_{h} K \subset k_{h}
\end{aligned}
$$

Considérons 1 'inéquation variationnelle :

$$
\begin{aligned}
& \sum_{q, r} a_{q r}\left(p_{h}^{q} u_{h}, p_{h}^{r} u_{h}-p_{h}^{r} v_{h}\right) \leqslant \sum_{r}\left(f_{r}, p_{h}^{r} u_{h}-p_{h}^{r} v_{h}\right) ; v_{h} \varepsilon k_{h} \\
& \text { On démontre alors le }
\end{aligned}
$$

COROLLAIRE 4-2.

Sous l'hypothèse (4-10), les solutions $u_{h}$ convergent vers la solution $u$ au sens suivant :

$$
\lim _{h=0}\left[\left\|u-p_{h}^{r} u_{h}\right\|_{r}^{2}=0\right.
$$

EXEMPLE 4-2. (ç. EXemple 3-2 du $\$ 3, n^{0} 3-3$ ).

Considérons les formes $b_{h}\left(u_{h} ; v_{h}, w_{h}\right)$ sur $v_{h}$, linéaires et continues en $v_{h}$ et $w_{h}$, vérifiant :
i) $b_{h}\left(u_{h} ; v_{h}, w_{h}\right) \geqslant c|| v_{h} \|_{h}^{2}$ si $u_{h}$ appartient à $k_{h}^{\rho}$ ii) $\left|b_{h}\left(u_{h}^{1} ; v_{h}, w_{h}\right)-b_{h}\left(u_{h}^{2} ; v_{h}, w_{h}\right)\right| \leqslant L|| u_{h}^{1}-u_{h}^{2}\left\|\left.\right|_{h}|| v_{h}||_{h}|| w_{h}\right\|_{h}$. 
et considérons la forme :

$$
a_{h}\left(u_{h} ; v_{h}\right)=b_{h}\left(u_{h} ; u_{h}, v_{h}\right)
$$

Supposons aussi qu'il existe $u_{h}^{0} \in k_{h}^{\rho}$ tel que :

(4-13) $\quad\left|b_{h}\left(u_{h}^{0} ; u_{h}^{0}, v_{h}\right)\right| \leqslant\left. M|| v_{h}\right|_{h} ; M$ indépendante de $v_{h}$.

On considère alors la méthode itérative suivante :

PROBLEME $\mathrm{P}_{\mathrm{h}}^{\mathrm{n}}$.

Chercher $u_{h}^{n}$ dans $K_{h}^{\rho}$ vërifiant :

(4-14) $b_{h}\left(u_{h}^{n-1} ; u_{h}^{n}, u_{h}^{n}-v_{h}\right) \leqslant\left(f_{h}, u_{h}^{n}-v_{h}\right)_{h}$ pour tout $v_{h}$ de $k_{h}$.

THEOREME 4-2.

Sous les hypotineses (4-4), (4-6), (4-7), (4-11), (4-12) et (4-13), si $\rho<\mathrm{cL}^{-1},\left\|\mathrm{f}_{\mathrm{h}}\right\|_{\times \mathrm{h}} \leqslant \mathrm{c} \rho$ et si 0 appartient à $\mathrm{K}$, les problèmes $\mathrm{P}_{\mathrm{h}}^{\mathrm{n}}, \mathrm{P}_{\mathrm{h}}$ et $\mathrm{P}$ admettent des solutions uniques $u_{h}^{n}$, $u_{h}$ et $u$ dans $K_{h}^{\rho}$ et $k$. Les soiutions $u_{h}^{n}$ convergent discrètement vers $u$ quand $h$ et $n^{-1}$ tendent vers 0 . $(4-15)\left\{\begin{array}{c}\left\|u_{h}^{n+1}-r_{h} u\right\|_{h}^{2} \leqslant(c-L \rho)^{-2}\left(\psi_{u}(h)+\varepsilon\left(f, f_{h}\right)+N L \rho \theta^{n}\right)^{2}+ \\ +2(c-L \rho)^{-1}\|f-A(u)\|_{*}\left\|u-p_{h} r_{h} u\right\|\end{array}\right.$ où $\theta=c^{-1} L \rho<1$

Si $u_{h}^{n}$ sont les solutions de l'inéquation restriction :

$(4-16) b\left(p_{h} u_{h}^{n-1} ; p_{h} u_{h}^{n} ; p_{h}\left(u_{h}^{n}-v_{h}\right)\right) \leqslant\left(f, p_{h}\left(u_{h}^{n}-v_{h}\right)\right) \forall v_{h} \varepsilon k_{h}$

Les erreurs vêrifient l'inégalité :

$(4-17)\left\{\begin{aligned}|| u-p_{h} u_{h}^{n+1} \|_{h}^{2} \leqslant & 2(c-L \rho)^{-1} \| f-B\left(p_{h} u_{h}^{n} ; p_{h} u_{h}^{n+1}\left\|_{*}|| u-p_{h} x_{h} u\right\|+\right. \\ & +(c-L \rho)^{-2} N^{2} \theta^{2 n}\end{aligned}\right.$

Le corollaire 3-2 du $\$ 3$ est un cas particulier de ce théorème. Nous savons déjà que la condition $S^{\prime}$ est satisfaite puisque :

(4-18) $\quad s_{h}\left(u_{h}, v_{h}\right) \geqslant(c-L \rho)\left\|u_{h}-v_{h}\right\|_{h}^{2}$

Nous en déduisons donc $l^{\prime}$ unicité des solutions des problèmes $P_{h}^{n}, P_{h}$ et $P$.

a) LES SOLUTIONS $u_{h}^{n}, u_{h}$ ET $u$ APPARTIENNENT NECESSAIREMENT A $k_{h}^{\rho}$ ET A $k^{\rho}$. En faisant $v_{h}=0$ dans les inéquations (4-14), on obtient : 
(4-19) $\quad\left\|u_{h}^{n}\right\|_{h} \leqslant c^{-1}\left\|f_{h}\right\|_{* h} \leqslant c^{-1} c \rho=\rho$.

On voit donc que si $u_{h}^{n-1}$ appartient à $K_{h}^{\rho}$, $u_{h}^{n}$ appartient aussi à $k_{h}^{\rho}$. De même, les solutions $u$ et $u_{h}$ appartiennent nécessairement à $K^{\rho}$ et $k_{h}^{\rho}$.

b) LES SOLUTIONS DES PROBLEMES $\mathrm{P}_{h}^{\mathrm{n}}, \mathrm{P}_{\mathrm{h}}$ ET $\mathrm{P}$ EXISTENT.

Puisque les solutions $u_{h}^{n-1}$ appartiennent à $K_{h}^{\rho}$ si elles existent, et puisque $u_{h}^{\circ}$ appartient à $k_{h}^{\rho}$, les solutions $u_{h}^{n}$ existent d'après (4-11) i) et le théorème de Stampacchia.

Nous allons montrer que les solutions $u_{h}^{n}$ convergent vers $u_{h}$. Ceci résultera de l'inégalité :

$$
\left\{\begin{aligned}
\left\|u_{h}^{n+1}-u_{h}^{n}\right\|_{h} & \leqslant\left(c^{-1} L \rho\right)\left\|u_{h}^{n}-u_{h}^{n-1}\right\|_{h} \leqslant \\
& \leqslant c^{-1}(c \rho+M)\left(c^{-1} L \rho\right)^{n}=N \theta^{n} \quad ; \quad \theta=c^{-1} L \rho<1
\end{aligned}\right.
$$

En effet, d'après (4-11) :

$$
c|| u_{h}^{n+1}-u_{h}^{n} \mid \|_{h}^{2} \leqslant b_{h}\left(u_{h}^{n} ; u_{h}^{n+1}-u_{h}^{n}, u_{h}^{n+1}-u_{h}^{n}\right) \leqslant
$$$$
\leqslant b_{h}\left(u_{h}^{n-1} ; u_{h}^{n}, u_{h}^{n+1}-u_{h}^{n}\right)-b_{h}\left(u_{h}^{n} ; u_{h}^{n}, u_{h}^{n+1}-u_{h}^{n}\right)
$$$$
\leqslant L|| u_{h}^{n}-u_{h}^{n-1}||_{h}|| u_{h}^{n}\left|\left\|_{h}|| u_{h}^{n+1}-u_{h}^{n}\right\|_{h} \leqslant L \rho\right|\left|u_{h}^{n+1}-u_{h}^{n}\right|\left\|_{h}|| u_{h}^{n}-u_{h}^{n-1} \mid\right\|_{h} \text {. }
$$

et d'après (4-12) :

$$
\begin{aligned}
& c|| u_{h}^{1}-u_{h}^{0} \|_{h}^{2} \leqslant b_{h}\left(u_{h}^{0} ; u_{h}^{1}-u_{h}^{0}, u_{h}^{1}-u_{h}^{0}\right) \leqslant\left(f_{h}, u_{h}^{1}-u_{h}^{0}\right)_{h}+ \\
& +b_{h}\left(u_{h}^{0} ; u_{h}^{0}, u_{h}^{1}-u_{h}^{0}\right) \leqslant(M+c \rho)|| u_{h}^{1}-u_{h}^{0} \|_{h}
\end{aligned}
$$

Pour tout $h$ fixé, on déduit de $(4-20)$ que la suite $u_{h}^{n}$ est une suite de Cauchy puisque $\theta=c^{-1} L \rho<1$, qui converge vers un élément $u_{h}$ de $v_{h}$, qui est solution de l'inéquation (4-14) puisque la forme $b_{h}\left(u_{h}, v_{h}, w_{h}\right)$ est continue par rapport aux trois variables.

c) LES SOLUTIONS $u_{h}^{n}$ CONVERGENT DISCRETEMENT VERS LA SOLUTION $u$.

En ajoutant et retranchant $b_{h}\left(u_{h}^{n-1} ; u_{h}^{n}, u_{h}^{n}-r_{h} u\right)$, on obtient 1'inégalité : $(4-21)\left\{\begin{array}{l}s_{h}\left(u_{h}^{n}, r_{h} u\right) \leqslant\left(\psi_{u}(h)+\varepsilon\left(f, f_{h}\right)+L \rho\left\|u_{h}^{n}-u_{h}^{n-1}\right\| \|_{h}\right)\left\|u_{h}-r_{h} u\right\|_{h} \\ +\|f-A(u)\|\left\|_{*}\right\| u-p_{h} r_{h} u \|\end{array}\right.$

La majoration de 1 'erreur (4-15) résulte alors de (4-18), (4-20) et (4-21). On obtient de manière analogue 1 'inégalité (4-17) dans le cas ou $A_{h}$ est la restriction de l'opérateur A. 
On démontre de la même manière la convergence discrète des solutions $u_{h}$ lonsque $l^{\prime}$ on prend pour opérateur $A_{h}$ la somme des restrictions partielles de l'opérateur A (cf. \$3, n 3-5, proposition 3-3).

Nous allons énoncer un théorème de convergence faible lorsque les formes $a_{h}\left(u_{h}, v_{h}\right)$ sont seulement monotones.

THEOREME 4-3.

Sous les hypothèses du théorème 1 du $\$ 4$, où la condition $S_{2}^{\prime}$ est remplacée par la condition $s_{3}^{\prime}$ :

$s^{\prime}\left\{\begin{array}{c}\text { i) } s_{h}\left(u_{h}, v_{h}\right) \geqslant 0 \\ \text { ii) }\left\|_{h}\right\|_{h} \|_{h}=\infty \\ s_{h}\left(u_{h}, r_{h} u_{0}\right) /\left\|u_{h}-r_{h} u_{0}\right\|_{h}=\infty \\ \text { iii) A est borné et } \underset{\lambda=0}{\lim } a(u-\lambda w ; v)=a(u, v)\end{array}\right.$

On peut extraire une sous-suite $u_{h}$ de la famille des solutions $u_{h}$ des problèmes $P_{h}$ convergeant faiblement vers une solution $u$ du problème $P$.

Le théorème de Hartmann-Stampacchia entrafne l'existence des solutions des problèmes $P$ et $P_{h}$. L'unicité a líeu si on suppose que :

(4-22) $\quad s(u, v)>0$ pour tout $u, v \varepsilon K ; u \neq v$.

La démonstration du théorème 3 du $\$ 4$ est analogue à celle du théorème $3-5$ du $\$ 3$. On déduit de $S_{3}^{\prime}$ ii) que $\left\|u_{h}\right\|_{h}$ est borné, donc que $\left\|p_{h} u_{h}\right\|$ l'est également. On peut. donc en extraire une sous-suite (encore notée) $p_{h} u_{h}$ qui converge faiblement vers un élément $u_{*}$ de $K$ (convexe fermé).

Montrons que $u_{*}$ est solution du problème $P$. Solt pour cela $v$ un élément arbitraire de $K$.

Puisque

$$
\begin{aligned}
0 \leqslant & s_{h}\left(u_{h}, r_{h} v\right) \leqslant\left(f_{h} ; u_{h}-r_{h} v\right)_{h}-\left(f, p_{h} u_{h}-p_{h} r_{h} v\right) \\
& +a\left(v ; p_{h} u_{h}-p_{h} r_{h} v\right)-a_{h}\left(r_{h} v ; u_{h}-r_{h} v\right) \\
& +\left(f, p_{h} u_{h}-p_{h} r_{h} v\right)-a\left(v ; p_{h} u_{h}-p_{h} r_{h} v\right)
\end{aligned}
$$

on en déduit qu'après passage à la limite, on obtient :

(4-23) $0 \leqslant\left(f, u_{*}-v\right)-a\left(v ; u_{*}-v\right) ; v \varepsilon K$

$D^{\prime}$ après le procédé de MINTY, $u_{*}$ est solution de (4-1). En effet, soit $v=u_{*}+\lambda\left(w-u_{*}\right)=\lambda w+(1-\lambda) u_{*}, 0 \leqslant \lambda \leqslant 1$ qui appartient au convexe $k$ si $w$ appartient à $K$. 
On déduit de (4-23) que :

$\lambda\left(A\left(u_{*}+\lambda\left(w-u_{*}\right), w-u_{*}\right) \geqslant \lambda\left(f, w-u_{*}\right)\right.$

En divisant par $\lambda>0$ et en faisant tendre $\lambda$ vers 0 , il résulte de $S_{3}^{\prime}$ ii) que :

$\left(A\left(u_{*}\right), u_{*}-w\right) \leqslant\left(f, u_{*}-w\right)$ pour tout $w$ de $K$, ce qui achève la démonstration du théorème.

On peut démontrer des théorèmes analogues lorsque 1 'on prend pour opérateur $A_{h}$ la somme des restrictions partielles d'un opérateur $A$ ou la restriction d'un opérateur A .

§5. APPROXIMATION DES SOLUTIONS DES EQUATIONS DIFFERENTIELLES-OPERATIONNELLES

Nous approchons dans ce varagraphe les solutions des équations différentiellesopérationnelles linéaires du $1^{\circ}$ ordre.

$$
\frac{d u}{d t}(t)+A(t) u(t)=f(t) ; u(0)=u_{0}
$$

où $A(t)$ sont des opérateurs d'un espace de Hilbert $V$ dans son dual, par les solutions $u_{h}(t)$ d'un système d'équations différentielles usuelles :

$$
\frac{d u_{h}}{d t}(t)+A_{h}(t) u_{h}(t)=\dot{I}_{h}(t) \quad ; \quad u_{h}(0)=u_{o h}
$$

où $A_{h}(t)$ sont des opérateurs $d^{\prime}$ un espace de dimension finie $v_{h}$.

Le $n^{\circ}$ 5-1 est consacré à quelques rappels et le théorème de convergence est démontré au $n^{\circ}$ 5-2.

Nous étudierons au chapitre II, \$5, l'approximation par rapport à la variable $t$ des solutions $u_{h}$ et (par suite) de la solution $u$.

Nous supposerons connus les $n^{\circ} 3-1$ à $3-3$ du $\$ 3$, les $n^{0} 1-6$ et $1-8$ du $\$ 1$, et le théorème 2-1 du $n^{0} 2-4$ du $\$ 2$.

5-1. RAPPELS SUR LES EQUATIONS DIFFERENTIELLES-OPERATIONNELLES DU $1^{e}$ ORDRE.

Nous rappelons dans ce numéro le théorème d'existence et d'unicité d'une solution d'une équation différentielle-opérationnelle (cf. J.L. LIONS [1]).

On se donne un triplet $A(t)$ dépendant de $t \in[0, T]$ (cf. Appendice). (5-1) $\mathrm{A}(\mathrm{t})=\{\mathrm{V}, \mathrm{H} ; \mathrm{a}(\mathrm{t} ; \mathrm{u}, \mathrm{v})\} ; t \varepsilon[0, \mathrm{~T}]$ vérifiant : 
$(5-2)\left\{\begin{array}{l}\text { i) } t \rightarrow a(t ; u, v) \text { est mesurable pour tout } u, v \text { fixé dans } v \\ \text { ii) }|a(t ; u, v)| \leqslant M|| u||\|v\| ; M \text { indépendante de } t(p \cdot p) \\ \text { iii) } \operatorname{Re} a(t ; v, v)+\lambda|v|^{2} \geqslant c|| v||^{2} ; c>0 ; v \varepsilon v\end{array}\right.$

On introduit d'autre part les espaces suivants:

$(5-3)\left\{\begin{array}{l}V=L^{2}(0, T ; V) ; V^{\prime}=L^{2}\left(0, T ; V^{\prime}\right) ; H=L^{2}(0, T ; H) \\ W=\left\{u \in V \text { telles que } u^{\prime}=\frac{d u}{d t} \varepsilon V^{\prime}\right\} \\ \phi=\{\phi \varepsilon W \text { telles que } \phi(T)=0\}\end{array}\right.$

L'espace $W$ est contenu dans l'espace $C(0, T ; H)$ des fonctions continues à valeurs dans $H$.

On se donne d'autre part :

$(5-4) \quad f \in V^{\prime} ; u_{0} \varepsilon M ; F=\left(f, u_{0}\right) \in X=V^{\prime} \times H$

Considérons les problèmes équivalents suivants :

PROBLEME P .

Chercher $u$ dans $w$ vérifiant : $\Lambda \mathrm{u}=\mathrm{F}$ dans $\mathrm{X}$ :

$$
\begin{array}{ll}
\frac{d u}{d t}+A(t) u=f(t) & \text { dans } V^{\prime} \\
u(0)=u_{0} & \text { dans } H
\end{array}
$$

PROBLEME $P^{\prime}$.

Chercher u dans $V$ vérifiant :

$$
\begin{aligned}
& E(u, \phi)=\int_{0}^{T}\left\{-\left(u, \phi^{\prime}\right)+a(t ; u, \phi)\right\} d t= \\
& =F(\phi)=\int_{0}^{T}(f, \phi) d t+\left(u_{0}, \phi(0)\right)
\end{aligned}
$$

pour toute fonction $\phi$ de $\Phi$.

Il existe une solution unique de ce problème :

THEOREME A .

Sous les hypothèses (5-2), $\Lambda$ est un isomorphisme de $w$ sur $X$. La solution u du problème $P$ vérifie l'égalité de l'énergie:

$(5-7)|u(t)|^{2}+2 \operatorname{Re} \int_{0}^{T} a(s ; u(s), u(s)) d s=\left|u_{0}\right|^{2}+2 \operatorname{Re} \int_{0}^{t}(f(s), u(0)) d s$. 
REMARQUE 5-1.

On peut toujours supposer dans l'hypothèses (5-2) iii) que $\lambda=0$, en faisant le changement de fonction inconnue $u^{*}=u e^{\lambda t}$. Par la suite, nous supposerons que $\lambda=0$.

\section{5-2. APPROXIMATION PAR DES SYSTEMES D'EQUATIONS DIFFERENTIELLES :}

Afin de fixer les notations et de montrer sur un exemple simple les méthodes que nous utiliserons (cf. $\$ 5, n^{\circ} 5-1$ ), nous allons étudier $1^{\prime}$ approximation des solutions des équations différentielles-opérationnelles par celles d'équations différentielles usuelles et démontrer un théorème de convergence forte dans l'espace $w$.

Nous utiliserons ici de façon essentielle la notion $a^{\prime \prime}$ upproximation autoadjointe $\left(\$ 1, \mathrm{n}^{\circ} 1-6\right)$ pour pouvoir construire des approximations de l'espace $\omega$ à partir d'approximations de 1 'espace $V$.

Rappelons qu'un premier exemple d'approximations autoadjointes (approximations de Galerkine) a été donné au $\$ 1, \mathrm{n}^{\circ} 1-8$. On trouvera au chapitre II, $\$ 3, \mathrm{n}^{\bullet} 3-2$ des exemples d'approximations autoadjointes des espaces de Sobolev $\mathrm{H}_{0}^{\mathrm{m}}(\Omega)$.

Rappelons que (cf. Appendice)

$$
\mathrm{V} \subset \mathbf{н} \subset \mathrm{V}^{\prime}
$$

Donnons-nous des approximations autoadjointes de $\mathrm{V}$ dans des espaces de dimension finie $V_{h}$ :

(5-8) $A_{h}(v)=\left(v, v_{h}, p_{h}, r_{h}^{*}\right) ; A_{h}\left(v^{\prime}\right)=\left(v^{\prime}, v_{h}, p_{h}, r_{h}^{*}\right)$

Nous désignerons par $\left(u_{h}, v_{h}\right)_{h}$ le produit scalaire identifiant $v_{h}$ et son dual. Rappelons que :

$$
\left(p_{h} u_{h}, v\right)=\left(u_{h}, r_{h}^{*} v\right)_{h} \text {. }
$$

Posons :

$(5-9) V_{h}=L^{2}\left(0, T ; V_{h}\right) ; V_{h}^{\prime}=L^{2}\left(0, T ; V_{h}^{\prime}\right) ; W_{h}=\left\{u \varepsilon V_{h}\right.$ telles que $\left.u^{\prime} \varepsilon V_{h}^{\prime}\right\}$

Donnons-nous une famille de triplets :

$$
A_{h}(t)=\left\{v_{h}, v_{h} ; a_{h}\left(t ; u_{h}, v_{h}\right)\right\}
$$

vérifiant les hypothèses $(5-2)$ et $F_{h}=\left(f_{h} \cdot u_{o h}\right) \varepsilon X_{h}=V_{h}^{\prime} \times v_{h}$

Considérons alors le : 
PROBLEME $\mathrm{P}_{\mathrm{h}}$.

Chercher $u_{h}$ dans $w_{h}$ vērifiant $\Lambda_{h} u_{h}=F_{h}$ dans $x_{h}$ :

(5-10) $\left\{\begin{array}{l}\text { i) } \frac{d u_{h}}{d t}+A_{h}(t) u_{h}=f_{h} \text { dans } v_{h}=V_{h}^{\prime} \\ \text { ii) } u_{h}(0)=u_{\text {oh }} \text { dans } v_{h} .\end{array}\right.$

Puisque $v_{h}$ est un espace de dimension finie et que la matrice est définie positive (d'après $(5-3)), u_{h}$ est la solution du problème de Cauchy pour un système d'équations différentielles linéaires.

Considérons alors les solutions uniques des problèmes $P$ et $P_{h}$ :

THEOREME 5-1.

Supposons que :

(5-11) $\lim _{h=0}\left[\int_{0}^{T}|| p_{h} v_{h}||_{h}^{2} d t\right]-1 / 2 \int_{0}^{T}\left|a_{h}\left(t ; r_{h}^{*}, v_{h}\right)-a\left(t ; u, p_{h} v_{h}\right)\right| d t=0$

(5-12) $\begin{cases}\text { i) } & \lim _{h=0}\left|u_{o h}-r_{h}^{*} u_{o}\right|_{h}=0 \\ \text { ii) } & \lim _{h=0}\left(\int_{0}^{T}|| p_{h} v_{h}||_{h}^{2} d t\right)^{-1 / 2} \int_{0}^{T}\left|\left(f_{h}(t), v_{h}\right)_{h}-\left(f, p_{h} v_{h}\right)\right| d t=0\end{cases}$

Les solutions $u_{h}$ convergent alors discrètement vers $u$ dans l'espace $w$ :

(5-13) $\lim _{h=0} \int_{0}^{T}\left\{|| r_{h}^{*} u-u_{h}\left\|_{h}^{2}+|| \frac{d}{d t}\left(r_{h}^{*} u-u_{h}\right)\right\|_{* h}^{2}\right\} d t=0$

et par suite :

(5-14) $\lim _{h=0} \sup _{t \varepsilon[0, T]}\left|r_{h}^{*} u(t)-u_{h}(t)\right|_{h}=0$.

Si l'on suppose de plus que les approximations autoadjointes $A_{h}(V)$ sont stables et convergentes, les solutions $u_{h}$ convergent vers $u$ dans $l^{\prime}$ espace $w$ :

(5-15) $\lim _{h=0} \int_{0}^{T}\left\{\left\|u-p_{h} u_{h}\right\|^{2}+\left\|\frac{d}{d t}\left(u-p_{h} u_{h}\right)\right\|_{x}^{2}\right\} d t=0$

et par suite :

(5-16) $\lim _{h=0} \sup _{t \varepsilon[0, T]}\left|u(t)-p_{h} u_{h}(t)\right|=0$

Nous allons démontrer la convergence discrète des solutions $u_{h}$ vers la 
solution $u$ à $1^{\prime}$ aide du théorème 2-1 du $\$ 2, \mathrm{n}^{\circ} 2-4$.

En effet, les hypothèses $(5,12)$ impliquent la convergence discrète de $f_{h}$ vers $f$ et de $u_{o h}$ vers $u_{0}$, donc de $F_{h}=\left(f_{h}, u_{o h}\right)$ vers $F=\left(f, u_{0}\right)$ dans 1 'espace $X_{h}$.

L'hypothèse $(5,11)$ implique la consistance des opérateurs $\Lambda_{h}$ à l'opérateur $\Lambda$ : En effet, puisque les approximations sont autoadjointes, nous avons :

(5-17) $\int_{0}^{T}\left\{\left(r_{h}^{*} u^{\prime}, v_{h}\right)_{h}-\left(u^{\prime}, p_{h} v_{h}\right)\right\} d t=0$

et 1 'hypothèse $(5,11)$ implique que dans $V_{h}^{\prime},\left\|r_{h}^{*} \Lambda u-\Lambda_{h} \mathbf{r}_{h}^{*} u\right\|_{V_{h}^{\prime}}$ tend vers 0 .

Il nous reste à montrer la stabilité des opérateurs $\Lambda_{h}^{-1}$, c'est-à-dire à montrer que si $\left\|f_{h}\right\|_{V_{h}^{\prime}}^{2}+\left|u_{o h}\right|_{h}^{2}$ est borné, 11 en est de même de $\left\|u_{h}\right\|_{V_{h}}^{2}+\left\|u_{h}^{\prime}\right\|_{V_{h}^{\prime}}^{2}$.

Soit $u_{h}$ la solution du problème $P_{h}$. Elle vérifie l'inégalité de l'énergie suivante :

$(5-18) \sup _{t}\left|u_{h}(t)\right|_{h}^{2}+\int_{0}^{T}|| u_{h}(t)||_{h}^{2} d t \leqslant k\left(\left.\int_{0}^{T}|| f_{h}(t)\right|_{\star h} ^{2}+\left|u_{o h}\right|_{h}^{2}\right)$

(obtenue en faisant le produit scalaire de (5-10) i) par $u_{h}$ et en intégrant de 0 à $t$ la partie réelle de l'expression obtenue).

On en déduit déjà que \|\|$_{h} \|_{V_{h}}$ est borné.

En faisant maintenant le produit scalaire de $(5-10)$ 1) par $V_{h}$, nous obtenons : $\left|\int_{0}^{T}\left(u_{h}^{\prime}, v_{h}\right)_{h} d t\right|=\left|\int_{0}^{T}\left\{\left(f_{h}, v_{h}\right)_{h}-a_{h}\left(t ; u_{h}, v_{h}\right)\right\} d t\right| \leqslant$

$\leqslant\left(\left\|f_{h}\right\|_{v_{h}^{\prime}}+\left\|u_{h}\right\|_{v_{h}}\right)\left\|v_{h}\right\|_{v_{h}} \leqslant k_{1}\left\|v_{h}\right\|_{v_{h}}$

d'où 1 'on déduit que $\left\|u_{h}^{\prime}\right\|_{V_{h}^{\prime}}$ est aussi borné, ce qui achève la démonstration de la stabilité.

Nous obtenons la convergence discrète des solutions $u_{h}$ vers la solution $u$ dans les espaces $W_{h}$. Si les approximations $A_{h}(v)$ sont stables et convergentes, on en déduit la convergence forte de $u_{h}$ vers $u$ dans l'espace $w_{h}$ (théorème 1-1 du \$1).

EXEMPLE : SCHEMAS RESTRICTIONS ; MAJORATION DE L'ERREUR.

Supposons que $V_{h}$ est un espace de dimension finie et que $p_{h}$ est injective. Posons :

$(5-19)-\left(u_{h}, v_{h}\right)=\left(p_{h} u_{h}, p_{h} v_{h}\right)$ 
et supposons que

(5-20) $\left\|p_{h} f_{h}\right\|_{*} \leqslant\left\|f_{h}\right\|_{* h}=\sup _{v_{h}} \frac{\left(p_{h} f_{h}, p_{h} v_{h}\right)}{\left\|p_{h} v_{h}\right\|}$

Alors, si $r_{h}^{*}$ est la transposée de $p_{h}$, définie par :

(5-21) $\left(p_{h} r_{h}^{*} f, p_{h} v_{h}\right)=\left(f, p_{h} v_{h}\right)$

les approximations autoadjointes $A_{h}(v)=\left(v, v_{h}, p_{h}, r_{h}^{*}\right)$ sont stables. On prendra alors :

$$
\left\{\begin{array}{l}
a_{h}\left(t ; u_{h}, v_{h}\right)=a\left(t ; p_{h} u_{h}, p_{h} v_{h}\right) \\
\left(f_{h}(t), v_{h}\right)_{h}=\left(f(t), p_{h} v_{h}\right) \\
u_{o h}=r_{h}^{*} u_{0} .
\end{array}\right.
$$

THEOREME 5-2.

Si l'on suppose que $(5,19),(5,20)$ et $(5,22)$ ont lieu, les erreurs $u-p_{h} u_{h}$ entre les solutions des problèmes $P$ et $P_{h}$ vérifient :

$$
\begin{aligned}
& \sup _{t}\left|u(t)-p_{h} u_{h}(t)\right|^{2}+\int_{0}^{T}\left\|u-p_{h} u_{h}\right\|^{2} d t \leqslant \\
& \quad \leqslant K\left(\left(\int_{0}^{T} \mid u-p_{h} r_{h} u \|^{2} d t\right)^{\frac{1}{2}}+\left|u_{0}-p_{h} r_{h}^{*} u_{0}\right|^{2}\right)
\end{aligned}
$$

quelle que soit la restriction $r_{h}$ de $v$ dans $v_{h}$.

En effet, on remarque que :

$$
\begin{aligned}
& \left(u^{\prime}-p_{h} u_{h}^{\prime}, u-p_{h} u_{h}\right)+a\left(t ; u-p_{h} u_{h}, u-p_{h} u_{h}\right)= \\
& =\left(u^{\prime}-p_{h} u_{h}^{\prime}, u-p_{h} r_{h} u\right)+a\left(t ; u-p_{h} u_{h}, u-p_{h} r_{h} u\right) \\
& =\left(F_{h}, u-p_{h} r_{h} u\right)
\end{aligned}
$$

Mais $F_{h}=f-p_{h} u^{\prime}{ }_{h}-A(t) p_{h} u_{h}$ est dans un borné de $L^{2}\left(0, T ; V^{\prime}\right)$. On en déduit, en intégrant deux fois la partie réelle de cette égalité, l'inégalité du théorème.

\section{EXEMPLE 5-1.}

Supposons que l'injection de V dans $\mathrm{B}$ soit compacte. Prenons pour approximations autoadjointes stables et convergentes, les approximations autoadjointes de Galerkine définies au $n^{\circ} 1-8$ du $\$ 1$ du Chapitre I . 
Si $\omega_{j}$ sont les vecteurs propres de l'opérateur $\Lambda$ défini par $((u, v))=(\Lambda u, v)$ on posera :

(5-23) $\quad p_{h} u_{h}=\sum_{j=1}^{n} u_{h}^{j} \omega^{j} \quad ; \quad h=n^{-1}$

Le système d'équations différentielles obtenu en faisant (5-22) s'écrit :

(5-24) $\frac{d}{d t} u_{h}^{j}(t)+\sum_{i=1}^{n} a\left(t ; \omega^{i}, \omega^{j}\right) u_{h}^{i}(t)=\left(f(t), \omega^{j}\right) ; 1 \leqslant j \leqslant n$.

COROLLAIRE 5-1.

Soit $u_{h}$ la solution de (5-24). Alors :

(5-25) $\lim _{h=0} \mid \int_{0}^{T}\left\{\left\|u(t)-p_{h} u_{h} \mid(t)\right\|^{2}+\left\|u^{\prime}(t)-\frac{d}{d t} p_{h} u_{h}\right\|_{*}^{2}\right\} d t=0$.

$(5-26)\left\{\begin{array}{l}\sup _{t}\left|u(t)-p_{h} u_{h}(t)\right|^{2}+\int_{0}^{T}|| u-\left.p_{h} u_{h}\right|^{2} d t \leqslant \\ \leqslant M\left[\left(\int_{0}^{T}|| u-\left.p_{h} r_{h}^{*} u\right|^{2} d t\right)^{1 / 2}+\left|u_{0}-p_{h} r_{h}^{*} u_{0}\right|^{2}\right]\end{array}\right.$

En effet, d'après 1 a proposition 5-1 du $n^{\circ} 1-8$ du $\$ 1$ du chapitre $I$, les hypothèses des théorèmes 5-1 et 5-2 sont satisfaites. 
\$1. APPROXIMATION DES ESPACES DE FonCtions CONTINUES ET DES ESPACES ${ }^{\mathrm{P}}$.

Nous construirons dans ce paragraphe une famille d'approximations de divers espaces de fonctions continues et des espaces $L^{P}$ à partir de la donnée de partitions de l'unité subordonnées à des recouvrements localement finis dont le module tend vers 0 . Ces approximations sont stables, convergentes, et, dans le cas des espaces $L^{P}$, autoadjointes. On fixe au $n^{\circ} 1-1$ les notations, on étudie au $n^{\circ} 1-21^{\prime}$ approximation des espaces de fonctions continues et au $n^{\circ} 1-31^{\prime}$ approximation des espaces ${ }_{L}^{P}$.

Ce paragraphe est indépendant du reste du chapitre et suppose connu le $\$ 1$ du chapitre I .

1-1. NOTATIONS.

Nous désignerons par $H$ l'un des espaces suivants de fonctions continues sur $\mathrm{R}^{\mathrm{n}}$.

- l'espace C

des fonctions continues sur $\mathbb{R}^{\mathrm{n}}$, muni de la topologie de la convergence compacte, définie par les semi-normes :

(1-1) $\quad\|\phi\|_{K}=\sup _{x \in K}|\phi(x)| ; K$ est un compact de $R^{n}$

- l'espace $C_{0}$

des fonctions continues nulles à l'infini, muni de la topologie de la convergence uniforme, définie par la norme :

(1-2) ||$\phi||=\sup _{\mathbf{x}}|\phi(x)|$

Si $\lambda$ est une fonction continue n'appartenant pas à $C_{0}$,

- e'espace $c_{\lambda}$ est le sous-espace des fonctions $\phi$ de $C_{0}$ décroissant plus vite que $\lambda^{-1}$, c'est-à-dire vérifiant :

(1-3) $|x|=\infty \quad|\lambda(x) \phi(x)|=0$ 
C'est un espace de Banach pour la norme

(1-4) $\quad\|\phi\|_{(\lambda)}=\|\phi||+\| \lambda \phi||$

Si $\Lambda$ est un ensemble de fonctions continues $\lambda$ n'appartenant pas à $C_{0}$,

- L'espace $C(\Lambda)$

est la limite projective ( $l^{\prime}$ intersection) des espaces $C_{\lambda}$. C'est un espace de

Fréchet si $\Lambda$ est dénombrable. (Par exemple,si $\Lambda$ est l'ensemble des polynômes, $C(\Lambda)$ est l'espace des fonctions continues à décroissance (polynomiale) rapide).

Considérons enfin

- l'espace $K$

des fonctions continues à support compact, limite inductive (réunion) des espaces de Banach $K_{K}$ des fonctions continues à support dans le compact $K$.

Rappelons que :

(1-5) $K \subset C(\Lambda) \subset c_{\lambda} \subset c_{0} \subset C$

Par dualité, nous obtenons :

(1-6) $\quad C^{\prime} \subset C^{\prime}{ }_{0} \subset C_{\lambda}^{\prime} \subset C^{\prime}(\Lambda) \subset K^{\prime}$

Ce sont respectivement les espaces des mesures à support compact, des mesures

bornées, des mesures croissant plus lentement que $\lambda$, plus lentement qu'une

fonction $\lambda$ de $\Lambda$, de toutes les mesures sur $\mathbb{R}^{n}$.

Nous introduirons auss $1,81 d \mu$ est une mesure et $1 \leqslant p \leqslant \infty$,

- l'espace $\mathrm{L}^{\mathrm{P}}(\mathrm{d} \mu)$

des fonctions de puissance $\mathrm{P}^{i e ̀ n e} \mathrm{~d} \mu$ - Intégrables, muni de la norme :

(1-7) $|\phi|_{P}=\left(\int \mid \phi(x)^{P} \mathrm{~d} \mu\right)^{1 / P}$

Si $\lambda$ est une fonction mesurable,

- l'espace $\mathrm{L}_{\lambda}^{\mathrm{P}}(\mathrm{d} \mu)$

est $1^{\prime}$ espace des fonctions $\phi$ de $L^{P}(d \mu)$ telles que $\lambda \phi$ appartiennent à $L^{P}(d \mu)$, muni de la norme :

(1-8) $\quad|\phi|_{P},(\lambda)=\left(|\phi|_{P}^{P}+|\lambda \phi|_{P}^{P}\right)^{1 / P}$

Nous allons construire des approximations des espaces $H$ et $L_{\lambda}^{P}(d \mu)$ à partir de partitions de $i$ 'unité de $\mathbb{R}^{n}$.

Si $R_{h}$ est un ensemble d'indices $\alpha$, nous désignerons par $\omega_{h}$ un recouvrement

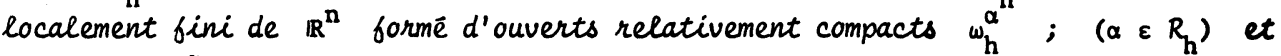
par $\sigma_{h}=\left(\sigma_{h}^{\alpha}\right)$ une partition de l'unité subordonnée au recouvrement $\omega_{h}$ : 
(1-9) $0 \leqslant \sigma_{h}^{\alpha}(x) \leqslant 1 ;$ support $\sigma_{h}^{\alpha} \subset \omega_{h}^{\alpha} ; \sum_{\alpha \in R_{h}} \sigma_{h}^{\alpha}(x)=1$

Nous appelerons module $\left|\omega_{h}\right|$ du recouvrement $\omega_{h}$ le plus petit pavé $\left|\omega_{h}\right|$ (centré à l'origine) tel que tout ouvert $\omega_{h}^{\alpha}$ du recouvrement $\omega_{h}$ soit contenu dans un translaté de $\left|\omega_{h}\right|$. Nous dirons que $\omega_{h}$ tend vers 0 si son module tend vers 0 .

Si $\phi$ est une fonction continue et $K$ un compact, nous désignerons par :

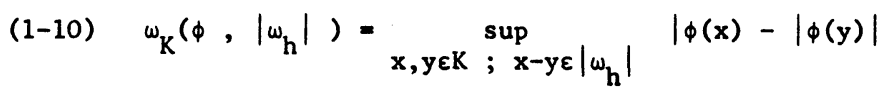
le moduce de continuité de $\phi$ sur $\left|\omega_{h}\right|$.

De même, si $\phi$ est une fonction de $L^{P}(d \mu)$, nous désignerons par : (1-11) $\omega_{P}\left(\phi,\left|\omega_{h}\right|\right)=\sup _{x-y \varepsilon\left|\omega_{h}\right|}\left|\int\right| \phi(x)-\left.\left.\phi(y)\right|^{P} d \mu(x)\right|^{1 / P}$ le $P$ - module de continuité de $\phi$ sur $\left|\omega_{h}\right|$.

\section{1-2. APPROXIMATIONS DISCRETES STABLES ET CONVERGENTES DES ESPACES DE FONCTIONS} CONTINUES.

Soit $H$ l'un des espaces $C, C_{0}, C_{\lambda}, C(\Lambda), K$. On associe à tout $h$ un ensemble $R_{h}$ d'indices $\alpha$, un recouvrement localement fini $\omega_{h}=\left(\omega_{h}^{\alpha}\right)$ et une partition de l'unité $\sigma_{h}=\left(\sigma_{h}^{\alpha}\right)$ subordonnée à ce recouvrement.

On considère ensuite des points $x_{h}^{\alpha}$ de $\omega_{h}^{\alpha}$ et on pose (1-12) $s_{h}^{\alpha}=\delta x_{h}^{\alpha}=$ mesure de Dirac au point $x_{h}^{\alpha}$.

Il en résulte que $\sigma_{h}=\left(\sigma_{h}^{\alpha}\right)$ est une suite d'éléments de $H$ et que $S_{h}=\left(s_{h}^{\alpha}\right)$ est une suite d'éléments de $H^{\prime}$. Nous allons montrer que ces suites définissent des approximations discrètes des espaces $H:$ (cf. chapitre $I, \$ 1, n^{0} 1-7$ ).

THEOREME $1-1$.

Supposons que :

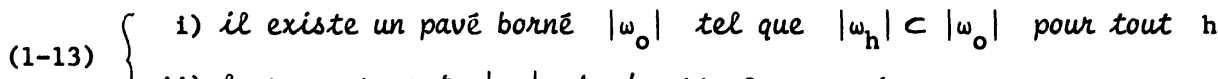
(ii) le recouvrement $\left|\omega_{h}\right|$ tend vers 0 avec $h$.

Les troncatures

(1-14) $\quad \mathrm{p}_{\mathrm{h}} \mathrm{r}_{\mathrm{h}}^{\phi}=\sum_{\alpha \in R_{\mathrm{h}}} \phi\left(\mathrm{x}_{\mathrm{h}}^{\alpha}\right) \sigma_{\mathrm{h}}^{\alpha}$

sont alors uniformément bornées dans $L(H, H)$ et tendent vers $l$ 'identité quand $h$ 
tend vers 0 .

Les approximations discrètes $A_{h}(H)=\left(H, \sigma_{h}, S_{h}\right)$ sont stables et convergentes.

REMARQUE 1-1.

Les espaces $H_{h}=r_{h} H$ sont les espaces suivants de suites scalaires sur $R_{h}$, analogues aux espaces $H$ :

- I'espace $c=c\left(R_{h}\right)$ de toutes les suites $u_{h}=\left(u_{h}^{\alpha}\right)$ sur $R_{h}$ si $H=C$.

- 1'espace $c_{0}=c_{0}\left(R_{h}\right)$ des suites $u_{h}$ telles que $|\alpha|=\infty u_{h}^{\alpha} \mid=0$ si $H=c_{0}$.
Si $\lambda_{h}^{\alpha}=\lambda\left(x_{h}^{\alpha}\right)$,

- 1 'espace $c_{\lambda_{h}}=c_{\lambda_{h}}\left(R_{h}\right)$ des suites $u_{h}$ de $c_{0}$ telles que $|\alpha|^{1 \text { im }}=\infty$
si $H=c_{\lambda}$

- l'espace $k=k\left(R_{h}\right)$ des suites à support fini sur $R_{h}$ si $H=K$.

Les prolongements $p_{h}$ et les restrictions $r_{h}$ sont définis par :

$p_{h} u_{h}=\sum_{\alpha \in R_{h}} u_{h}^{\alpha} \sigma_{h}^{\alpha} \quad ; \quad r_{h} \phi=\left(\phi\left(x_{h}^{\alpha}\right)_{\alpha \varepsilon R_{h}}\right.$.

Posons :

(1-15) $\quad\left(f_{h}, u_{h}\right)_{h}=\sum_{\alpha \varepsilon R_{h}} f_{h}^{\alpha} u_{h}^{\alpha}$

Les espaces $H_{h}^{\prime}=\left(I_{h} H\right)^{\prime}$ sont respectivement $l^{\prime}$ espace $k=c^{\prime}$, l'espace $1^{\infty}=c_{0}^{\prime}$ des suites bornées sur $R_{h}, l^{\prime}$ espace $c_{\lambda_{h}}^{\prime}$ des suites à croissance plus lente que la suite $\lambda_{h}$, l'espace $c=k^{\prime}$ de toutes les suites sur $R_{h}$. Les approximations duales sont définies par :

(1-16) $p_{h}^{*} f_{h}=\sum_{\alpha} f_{h}^{\alpha} \delta x_{h}^{\alpha} ; r_{h}^{*}(d \mu)=\left(\int \sigma_{h}^{\alpha}(x) d \mu\right)_{\alpha \varepsilon R_{h}}$

Ces approximations sont stables et falblement convergentes. (cf. Chapitre I, \$1 $\left.n^{\circ} 1-5\right)$. Si $f_{h}=r_{h}^{*}(d \mu)$, nous obtenons des formules de quadrature de la mesure $d \mu$ qui sont convergentes : $\left(p_{h}^{*} r_{h}^{*}(d \mu), \phi\right)$ converge vers $(d \mu, \phi)=\int \phi d \mu$.

S'il existe des points $x_{h}^{\alpha}$ tels que :

$$
\sigma_{h}^{\alpha}\left(x_{h}^{\beta}\right)=\left\{\begin{array}{lll}
0 & \text { si } & \beta \neq \alpha \\
1 & \text { si } & \beta=\alpha
\end{array}\right.
$$

les approximations ainsi obtemues sont des interpolations (chap I, \$1, proposition 1-4) et les troncatures $p_{h} r_{h}$ sont des projecteurs de $H$. 
DEMONSTRATION DU THEOREME 1-1.

Soit $K$ un compact de $\mathbb{R}^{\mathrm{n}}$; nous poserons

(1-18) $R_{h}(K)=\left\{\alpha \in R_{h}\right.$ tels que $\left.K \cap \omega_{h}^{\alpha} \neq \phi\right\} ; \sigma_{h}^{K}=\sum_{\alpha \in R_{h}(K)} \sigma_{h}^{\alpha}$

L'ensemble $R_{h}(K)$ est fini puisque $\omega_{h}$ est localement fini et les fonctions $\sigma_{h}^{K}$ sont positives, plus petites que 1 , à support contenu dans le compact :

$$
\mathrm{K}_{\mathrm{h}}=\bigcup_{\alpha \in R_{\mathrm{h}}(\mathrm{K})} \overline{\omega_{\mathrm{h}}^{\alpha}}
$$

D'après l'hypothèse (1-13) 1$)$, les compacts $K_{h}$ sont contenus dans un compact fixe $K_{0}$.

- Cas où $H=C$.

Montrons la stabilité : pour tout compact $K$, 11 existe $K_{0}$ tel que

$(1-20) \sup _{h} \sup _{x \in K}\left|p_{h} r_{h} \phi(x)\right| \leqslant \sup _{x \varepsilon K_{0}}|\phi(x)|$

Cette inégalité résulte des inégalités suivantes :

$$
\begin{aligned}
& \sup _{K}\left|p_{h} r_{h} \phi(x)\right|=\sup _{K}\left|\sum_{\alpha \in R_{h}(K)} \phi\left(x_{h}^{\alpha}\right) \sigma_{h}^{\alpha}(x)\right| \leqslant \\
& \leqslant \sup _{\alpha \in R_{h}(K)}\left|\phi\left(x_{h}^{\alpha}\right)\right| \sup _{K} \sigma_{h}^{K}(x) \leqslant \sup _{x \in K_{0}}|\phi(x)| .
\end{aligned}
$$

La convergence résulte de l'inégalité :

(1-21) Pour tout compact $K, \sup _{K}\left|\phi-p_{h} r_{h} \phi\right| \leqslant \omega_{K}\left(\phi,\left|\omega_{h}\right|\right)$ et de 1'hypothèse (1-13) ii). Pour établir (1-21), on écrit que $\phi(x)=\sum_{\alpha \in R_{h}} \phi(x) \sigma_{h}^{\alpha}(x)$ puisque
$\sigma_{h}$ est une partition de l'unité.

On en déduit que :

$$
\begin{gathered}
\sup _{K}\left|\phi-p_{h} x_{h} \phi\right| \leqslant \sup _{K_{h}} \sum_{\alpha \in R_{h}(K)}\left|\phi(x)-\phi\left(x_{h}^{\alpha}\right)\right| \sigma_{h}^{\alpha}(x) \leqslant \\
\leqslant \sup _{\alpha \varepsilon R_{h}(K)} \sup _{x \in K \cap \omega_{h}^{\alpha}}\left|\phi(x)-\phi\left(x_{h}^{\alpha}\right)\right| \leqslant \omega_{K}\left(\phi,\left|\omega_{h}\right|\right)
\end{gathered}
$$

- Cas où $H=K$.

La stabilité résulte de (1-21): Si les fonctions $\phi$ ont leur support dans $K$, les troncatures $P_{h} r_{h}^{\phi}$ ont leur support dans $K_{0}$. La convergence résulte alors de $(1-21)$.

- Cas oũ $H=C_{0}$.

I1 faut tout d'abord démontrer que si $\phi$ appartient à $C_{0}, p_{h} r_{h} \phi$ appartient à 
$c_{0}, c^{\prime}$ est-à-dire que

(1-22) $\underset{|x|=\infty}{11 m}\left|p_{h} r_{h} \phi(x)\right|=0$

Pour tout $\varepsilon>0$, $i 1$ existe un compact $K$ tel que :

$\sup _{x \notin K}|\phi(x)| \leqslant \varepsilon \quad$, donc $\sup _{\alpha \notin R_{h}(K)}\left|\phi\left(x_{h}^{\alpha}\right)\right| \leqslant \varepsilon$

Donc, puisque $K_{h}$ est contenu dans $k_{0}$ :

$\sup _{x \notin K_{0}}\left|p_{h} r_{h} \phi(x)\right| \leqslant \sup _{\alpha \notin R_{h}\left(K_{0}\right)}\left|\phi\left(x_{h}^{\alpha}\right)\right| \leqslant \varepsilon$

ce qui montre (1-22) et la stabilité des approximations, puisque $\mathrm{K}_{0}$ ne dépend pas de $h$. On démontre la convergence de façon analogue.

- Cas où $H=C_{\lambda}$ et où $H=C(\Lambda)$

On remarque qu'il suffit de démontrer que (pour tout $\lambda$ de $\Lambda$ )

$(1-23)\left\{\begin{aligned} & \text { i) } \lim _{|x|=\infty}(1+|\lambda(x)|)\left|p_{h} r_{h} \phi(x)\right|=0 \\ & \text { ii) } \sup _{h} \sup _{x}(1+|\lambda(x)|)\left|p_{h} r_{h} \phi(x)\right| \text { est borné } \\ & \text { iii) } \lim _{h=0} \sup _{x}(1+|\lambda(x)|)\left|p_{h} r_{h} \phi(x)-\phi(x)\right|=0\end{aligned}\right.$

Prenons, pour simplifier, pour point $x_{h}^{\alpha}$ le point où $\lambda(x)$ atteint son maximum sur $\omega_{h}^{\alpha}$. Posons alors :

(1-24) $\quad \lambda_{h}^{\alpha}=\lambda\left(x_{h}^{\alpha}\right) ; \rho_{h}^{\alpha}(x)=\frac{1+|\lambda(x)|}{1+\left|\lambda_{h}^{\alpha}\right|} \quad \sigma_{h}^{\alpha}(x) \leqslant 1$

On vérifie que :

$(1-25)(1+|\lambda|) p_{h} r_{h} \phi=\sum_{\alpha}\left(1+\left|\lambda_{h}^{\alpha}\right|\right) \phi\left(x_{h}^{\alpha}\right) \rho_{h}^{\alpha}$

Puisque $\sup _{\omega_{h}^{\alpha}} \frac{1+|\lambda|(x)}{1+\left|\lambda_{h}^{\alpha}\right|} \leqslant 1$ et puisque $(1+|\lambda|) \phi \varepsilon c_{0}$, on montre comme

précédemment que pour tout $\varepsilon$, 11 existe un compact $K$ tel que :

$\sup _{x \notin K}(1+|\lambda|)\left|p_{h} r_{h} \phi\right| \leqslant \sup _{x \notin K}(1+|\lambda|)|\phi| \leqslant \varepsilon$

ce qui implique $(1-23)$ 1). D'autre part, 11 résulte de $(1-20)$ que :

$$
\sup _{K_{0}}(1+|\lambda|)\left|p_{h} r_{h} \phi\right| \leqslant \sup _{K_{1}}(1+|\lambda|)|\phi|
$$


Ces deux dernières inégalités entrafnent (1-23) ii).

Enfin, puisque

$$
(1+|\lambda|)\left(\phi-p_{h} r_{h}^{\phi}\right)=\sum_{\alpha}\left(\phi(x)-\phi\left(x_{h}^{\alpha}\right)\right)(1+|\lambda|) \sigma_{h}^{\alpha}
$$

nous en déduisons que pour tout $\varepsilon$, il existe un compact $K$ et $h_{0}$ tel que :

$$
\left.\sup _{x \notin K}(1+|\lambda|) \mid \phi-p_{h} r_{h} \phi\right) \mid \leqslant \frac{\varepsilon}{2}
$$$$
\left.\sup _{x \varepsilon K}(1+|\lambda|) \mid \phi-p_{h} r_{h} \phi\right) \mid \leqslant \sup _{K}(1+|\lambda|) \omega_{K}\left(\phi,\left|\omega_{h}\right|\right) \leqslant \frac{\varepsilon}{2}
$$

ce qui entrafne (1-23) iii).

REMARQUE $1-2$.

A 1'aide de la proposition 1-7 du chapitre I, $\$ 1, n^{\bullet} 1-10$, on peut construire d'autres approximations des espaces $H$.

Par exemple, si $\rho$ est une fonction positive à support compact de masse totale égale à 1 , on prend pour opérateur $P_{h}$ l'opérateur de convolution par

$$
\rho_{h}(x)=\frac{1}{h} \rho\left(\frac{x}{h}\right) \text {. }
$$

Dans ce cas :

(1-26) $\quad P_{h} P_{h} r_{h}^{\phi}=\sum_{\alpha} \phi\left(x_{h}^{\alpha}\right) \rho_{h}^{*} \sigma_{h}^{\alpha}(x)$

et les hypothèses de la proposition 1-7 sont satisfaites puisque $\rho_{h}$ converge vers $\delta$. Si la fonction $\rho$ est $q$-fois différentiable, $\phi$ est alors approchée par des fonctions q-fois différentiables.

Considérons maintenant une suite de fonctions $\gamma_{h}$ à support compact $r_{h}$, uniformément bornées dans $C_{0}$ et convergeant vers 1 dans $C$. Prenons pour opérateur $R_{h}$ les opérateurs de multiplication par $\gamma_{h}$. Dans ce cas

(1-27) $p_{h} r_{h} R_{h} \phi=\sum \phi\left(x_{h}^{\alpha}\right) \gamma_{h}\left(x_{h}^{\alpha}\right) \sigma_{h}^{\alpha}(x)$

est une combinaison linéaire finie des fonctions $\sigma_{h}^{\alpha}$. Si $N(h)$ est le nombre des points $x_{h}^{\alpha}$ de $r_{h}$, les espaces $r_{h} R_{h} H$ sont des espaces de dimension finie $c^{\mathrm{N}(\mathrm{h})}$.

1-3. APPROXIMATIONS DES ESPACES ${ }_{L}^{P}(d \mu)$.

Soit $d \mu$ une mesure sur $\mathbb{R}^{n}$ et $L^{P}(d \mu) \quad l^{\prime}$ 'espace des fonctions de puissance $P^{i e ̀ m e} d \mu$ intégrables. Nous allons construire des approximations autoadjointes (cf. chapitre $\left.I, 51, n^{\circ} 1-6\right)$ stables et convergentes des espaces ${ }_{L}^{P}(d \mu) ~ a ̀$ 
partir de partitions de l'unité de $\mathrm{R}^{\mathrm{n}}$.

On associe à tout $h$ un ensemble $R_{h}$ d'indices $\alpha$, un recouvrement localement fini $\omega_{h}=\left(\omega_{h}^{\alpha}\right)$ et une partition de l'unité $\sigma_{h}=\left(\sigma_{h}^{\alpha}\right)$ subordonnée à ce recouvrement, formée de fonctions $\sigma_{h}^{\alpha}$ de $L^{\infty}(d \mu)$. Puisque les $\sigma_{h}^{\alpha}$ sont à support compact, les $\sigma_{h}^{\alpha}$ appartiennent aussi à $L^{P}(d \mu)$ pour tout $P \geqslant 1$.

Posons

(1-28) $\quad \theta_{h}^{\alpha}=\int \sigma_{h}^{\alpha}(x) d \mu$

et désignons par $\ell_{h}^{P}(d \mu)$ l'espace des suites $u_{h}=\left(u_{h}^{\alpha}\right)$ sur $R_{h}$ vérifiant :

(1-29) $\quad\left|u_{h}\right|_{h, P}=\left(\sum_{\alpha \in R_{h}}\left|u_{h}^{\alpha}\right|^{P}\left|\theta_{h}^{\alpha}\right|^{1-P / 2}\right)^{1 / P}<+\infty$

Considérons alors :

$(1-30)\left\{\begin{array}{l}p_{h} u_{h}=\sum_{\alpha} u_{h}^{\alpha}\left(\theta_{h}^{\alpha}\right)^{-1 / 2} \sigma_{h}^{\alpha} \\ r_{h}^{*} \phi=\left(\left(\theta_{h}^{\alpha}\right)^{-1 / 2} \int \phi(x) \sigma_{h}^{\alpha}(x) d \mu\right)_{\alpha}\end{array}\right.$

I1 est clair que la forme sesquilinéaire

(1-31) $\quad\left(f_{h}, u_{h}\right)_{h}=\sum_{\alpha} f_{h}^{\alpha} u_{h}^{\alpha}$

met en dualíté les espaces $\ell_{h}^{P}(d \mu)$ et $\ell_{h}^{P^{\prime}}(d \mu)$ avec $\frac{1}{P}+\frac{1}{P^{T}}=1$, puisque, d'après l'inégalité de Hölder :

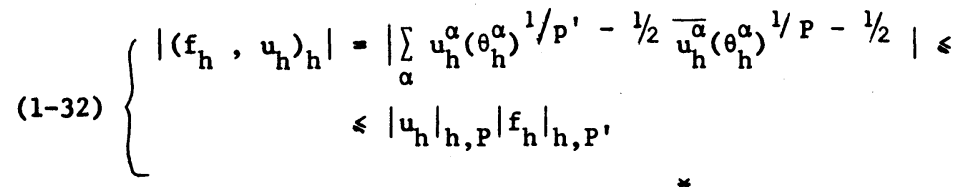

Il est alors clair que la restriction $r_{h}^{*}$ est la transposée du prolongement $\mathbf{p}_{\mathbf{h}} \cdot$

THEOREME 1-2.

Sous l'hypothese (1-13), les approximations discrètes autoadjointes

$A_{h}\left(L^{P}(d \mu)\right)=\left(L^{P}(d \mu), e_{h}^{P}(d \mu), P_{h}, r_{h}^{*}\right)=\left(L^{P}(d \mu),\left(\theta_{h}^{\alpha}\right)^{-1 / 2} \sigma_{h}^{\alpha}\right)$

de l'espace $L^{P}(d \mu)$ sont stables et convergentes :

(1-33)

1) $\left|p_{h} u_{h}\right|_{P} \leqslant\left|u_{h}\right|_{h, P} ;\left|r_{h}^{*} \phi\right|_{h, P} \leqslant|\phi|_{P}$

(ii) $\left|\phi-p_{h} x_{h}^{*} \phi\right|_{P} \leqslant \omega_{P}\left(\phi,\left|\omega_{h}\right|\right)$ 
- Les prolongements sont stables.

D'après l'inégalité de Hölder,

(1-34) $\left|\sum_{\alpha} \zeta^{\alpha} n^{\alpha}\right| \leqslant\left(\sum_{\alpha}\left|\zeta^{\alpha}\right|^{P} \eta^{\alpha}\right)^{1 / P}\left(\sum_{\alpha} n^{\alpha}\right)^{1 / P^{\prime}}$

et puisque $\sigma_{h}$ est une partition de l'unité, nous avons presque partout :

$\left|p_{h} u_{h}\right|^{P} \leqslant \sum\left|u_{h}^{\alpha}\right|^{P}\left|\theta_{h}^{\alpha}\right|^{-P / 2} \sigma_{h}^{\alpha}(x)$

d'où nous en déduisons la stabilité des prolongements.

- Les restrictions sont stables:

$$
\left|r_{h}^{*} \phi\right|_{h, P}^{P}=\sum_{\alpha}\left|\theta_{h}^{\alpha}\right|^{1-P}\left|\int \phi(x) \sigma_{h}^{\alpha}(x) d \mu\right|^{P}
$$

Mais, d'après l'inégalité de Hölder,

$\left|\int \phi(x) \sigma_{h}^{\alpha}(x) d \mu\right|^{P} \leqslant \int\left|\phi(x){ }^{P} \sigma_{h}^{\alpha}(x)\right| d \mu\left|\theta_{h}^{\alpha}\right|^{P / P^{\prime}}$

Puisque $1-\mathrm{P}-\mathrm{P} / \mathrm{P}^{\prime}=0$, 11 en résulte que :

$\left|\mathrm{r}_{\mathrm{h}}^{*} \phi\right|_{\mathrm{h}, \mathrm{P}}^{\mathrm{P}} \leqslant \int|\phi(\mathrm{x})|^{\mathrm{P}}\left(\sum_{\alpha} \sigma_{\mathrm{h}}^{\alpha}(\mathrm{x})\right) \mathrm{d} \mu=\int|\phi(\mathrm{x})|^{\mathrm{P}} \mathrm{d} \mu$.

- Les approximations sont convergentes :

On remarque que :

(1-35) $\phi(x)=\sum_{\alpha} \phi(x) \sigma_{h}^{\alpha}(x)=\sum_{\alpha}\left(\theta_{h}^{\alpha}\right)^{-1} \int_{h} \phi(x) d \mu(y) \sigma_{h}^{\alpha}(x)$

On en déduit que :

(1-36) $\phi-\mathrm{p}_{\mathrm{h}} \mathrm{r}_{\mathrm{h}}^{*} \phi=\sum_{\alpha}\left[\int[\phi(x)-\phi(y)] \sigma_{h}^{\alpha}(y) d \mu(y)\right]\left(\theta_{h}^{\alpha}\right)^{-1} \sigma_{h}^{\alpha}(x)$

Puisque les troncatures $\mathrm{p}_{h} \mathrm{r}_{h}^{*}$ sont dans un équicontinu de $L\left(L^{P}(d \mu), L^{P}(d \mu)\right)$, 11 suffit de montrer que $p_{h} r_{h}^{*} \phi$ converge vers $\phi$ pour toute fonction continue. Dans ce cas, en utilisant l'inégalité de la moyenne, nous obtenons :

$$
\begin{aligned}
\int\left|\phi-p_{h} r_{h}^{x} \phi\right|^{P} d \mu & \leqslant \sum_{\alpha} \int d \mu \sigma_{h}^{\alpha}(x) \sup _{x-y \varepsilon \omega_{h}^{\alpha}}|\phi(x)-\phi(y)|^{P} \\
& \leqslant \omega_{p}^{P}\left(\phi,\left|\omega_{h}\right|\right) .
\end{aligned}
$$


REMARQUE 1-3. APPROXIMATION DES ESPACES $L_{\lambda}^{P}(d \mu)$.

Soit $\lambda$ une fonction mesurable. Désignons par $\lambda_{h}^{\alpha}$ et $\bar{\lambda}_{h}^{\alpha}$ les bornes essentlelles inférieures et supérieures de $|\lambda(x)|$ sur $\omega_{h}^{\alpha}$ :

(1-37) $\quad_{h}^{\alpha} \leqslant|\lambda(x)| \leqslant \bar{\lambda}_{h}^{\alpha} \quad$ presque partout sur $\omega_{h}^{\alpha}$.

Désignons par $e_{\lambda ; h}^{P}(d \mu)$ l'espace des suites $u_{h}=\left(u_{h}^{\alpha}\right)$ telles que

(1-38) $\left|u_{h}\right|_{h, P,(\lambda)}=\left(\sum_{\alpha}\left|u_{h}^{\alpha}\right|^{P}\left(1+\left|\bar{\lambda}_{h}^{\alpha}\right|^{P}\right)\left|\theta_{h}^{\alpha}\right|^{1-P / 2}\right)^{1 / P}<+\infty$.

COROLLAIRE 1-1.

Sous les hypotheses $(1-13)$ et :

$(1-39)\left(1+\left|\bar{\lambda}_{h}^{\alpha}\right|^{P}\right)\left(1+\left|\lambda_{-h}^{\alpha}\right|^{P}\right)^{-1} \leqslant M$ indeppendant de $\alpha$ et de $h$, les approximations $A_{h}\left(L_{\lambda}^{P}(d \mu)=\left(I_{\lambda}^{P}(d \mu), e_{\lambda, \lambda}^{P}(d \mu), P_{h}, r_{h}^{*}\right)\right.$ sont stables et convergentes.

La stabilité des $p_{h}$ se montre comme précédemment. Vérifions la stabilité des restrictions $\mathbf{r}_{h}^{*}$ :

$$
\begin{aligned}
\left|r_{h}^{*} \phi\right|_{h, P, \lambda}^{P} & =\sum_{\alpha}\left|\theta_{h}^{\alpha}\right|^{1-P}\left(1+\left|\bar{\lambda}_{h}^{\alpha}\right|^{P}\right)\left|\int_{\phi}(x) \sigma_{h}^{\alpha}(x) d \mu\right|^{P} \\
& \leqslant M \sum_{\alpha}\left(1+\left|\lambda_{h}^{\alpha}\right|^{P}\right) \int|\phi(x)|^{P} \sigma_{h}^{\alpha}(x) d \mu \\
& \leqslant M \sum_{\alpha} \int\left(1+|\lambda(x)|^{P}\right)|\phi(x)|^{P} \sigma_{h}^{\alpha}(x) d \mu .
\end{aligned}
$$

La convergence des approximations s'établit comme précédemment.

§2. APPROXIMATION DES ESPACES DE SOBOLEV SUR $\mathrm{R}^{\mathrm{n}}$.

Nous allons construire des approximations des espaces de Sobolev $\mathrm{W}^{\mathrm{m}, \mathrm{P}}$ des (classes de) fonctions de puissance $P^{\text {lè̀me }}$ sommables sur $R^{n}$ dont les dértvées (au sens des distributions) jusqu'à $l^{\prime}$ ordre $m>0$ appartiennent à $L^{P}$. C'est un espace de Banach, réflexif si $1<P<\infty$, pour la norme :

$\left.|| \phi\right|_{m, P}=\left(\sum_{|q|_{\leqslant m}}\left|D^{q} \phi\right|_{P}^{P}\right)^{1 / P} ;|\phi|_{P}=\left(\int|\phi(x)|^{P} d x\right)^{1 / P}$

(nous poserons $W^{0, P}=L^{P}, W^{m, 2}=H^{m}$ ).

Nous construirons des approximations de ces espaces à partir de partitions de 
l'unité formée de translatées de puissances de convolutions des fonctions caractéristiques $x_{h}$ du cube $(-h, 0)^{n}$. En effet, les dérivées de telles fonctions sont des quotients différentiels.

On étudie ces fonctions au $n^{\circ}$ 2-1 et le théorème 2-1 du $n^{0} 2-2$ énonce les propriétés de ces approximations et leurs conséquences immédiates (inégalités de Sobolev discrètes). On généralise cette construction au $n^{\circ}$ 2-3 pour obtenir des approximations des espaces de distributions et des domaines de générateurs infinitésimaux de groupes continus d'opérateurs. On suppose connu le $\$ 1$ du chapitre I.

\section{2-1. PUISSANCES DE CONVOLUTION DES FONCTIONS CARACTERISTIQUES.}

L'approximation des opérateurs de dérivation par les différences finies fait jouer un rôle particulier aux fonctions caractéristiques et à leurs puissances de convolution. Nous groupons dans ce numéro quelques unes de leurs propriétés. Fixons tout d'abord quelques notations.

Nous désignerons par $q=\left(q_{1}, \ldots, q_{n}\right), K=\left(K_{1}, \ldots, K_{n}\right)$ des multientiers; en particulier, $(m)=(m, \ldots, m)$ est le multientier dont les composantes sont égales à $m, e_{i}=(0, \ldots, 0,1, \ldots, 0)$ est le multientier dont la $i^{\text {ème }}$ composante est égale à 1 , les autres étant nulles. Nous désignerons par $\alpha=\left(\alpha_{1}, \ldots, \alpha_{n}\right), \beta=\left(\beta_{1}, \ldots, \beta_{n}\right)$ les multientiers positifs ou négatifs de $\mathrm{z}^{\mathrm{n}}$

Nous utiliserons les notations classiques, par exemple :

$p \leqslant q$ si $p_{1} \leqslant q_{1}$ pour tout $i^{\prime},|q|=\sum_{i}\left|q_{1}\right|, q !=q_{1} ! \ldots q_{n} !$, etc...

Si $\sigma$ est une fonction, nous poserons, si $h=\left(h_{1}, \ldots, h_{n}\right), h_{1} \geqslant 0$ :

$$
\sigma_{h}(x)=\frac{1}{h} \sigma\left(\frac{x}{h}\right)=\frac{1}{h_{1} h_{2} \ldots h_{n}} \sigma\left(\frac{x_{1}}{h_{1}}, \ldots, \frac{x_{n}}{h_{n}}\right)
$$

et nous remarquons que :

$(\sigma * \rho)_{h}=\sigma_{h} * \rho_{h} ; \quad \rho_{h}^{\alpha}=(\delta(\alpha) * \rho)_{h}=\delta(\alpha h) * \rho_{h}=\frac{1}{h} \rho\left(\frac{x}{h}-\alpha\right)$

Enfin, nous désignerons par $x$ la fonction caractéristique du cube $(-1,0)^{\mathfrak{n}}$, par $x_{K}=x * \ldots * x$ sa puissance $K^{i e ̀ m e}$ de convolution par $x_{K}^{\alpha}=\delta(\alpha) * x_{K}$ sa translatée d'ordre $\alpha=\left(\alpha_{1}, \ldots, \alpha_{n}\right)$.

Nous poserons $x_{K}=x_{K}^{0}$ et $x_{0}=\delta$

En résumé :

$(2-1) \quad x_{K, h}^{\alpha}(x)=\underbrace{n}_{i=1} x_{K_{i}, h_{i}}^{\alpha_{1}}\left(x_{i}\right)=\underbrace{n}_{i=1} \frac{1}{h_{1}} x_{K_{i}}\left(\frac{x_{i}}{h_{1}}-\alpha_{i}\right)$ 
est une fonction de support.

$(2-2) \prod_{i=1}^{n}\left[\left(\alpha_{i}-K_{i}\right) h_{i}, \alpha_{i} h_{i}\right]=\omega_{K-(1), h}^{\alpha}$

Nous poserons aussi :

(2-3) $\bar{x}_{h}=T_{h} x_{h} \quad ; \quad \hat{x}_{h}=T_{h / 2} x_{h}=T_{-h / 2} \bar{x}_{h}$

LES QUOTIENTS DIFFERENTIELS $\nabla_{\mathrm{h}}^{\mathrm{q}}$.

Nous désignerons les quotients différentiels usuels par :

(2-4) $\nabla_{h_{i}}=h_{i}^{-1}\left(\delta\left(-h_{i}\right)-\delta\right) * ; \nabla_{h}^{q}=\nabla_{h_{1}}^{q_{1}} \ldots \nabla_{h_{n}}^{q_{n}}$ si $q=\left(q_{1}, \ldots, q_{n}\right)$

On remarque alors que :

(2-5) $\quad \nabla_{h}^{q}=D^{q} x_{q, h^{*}}=h^{-q} \sum_{k \leqslant q}(-1)^{|k|} C_{q}^{k} \delta(k-q) h *$

On introduit de même :

$\bar{\nabla}_{h}^{q}=D^{q} \bar{x}_{q, h^{*}} \quad ; \quad \hat{\nabla}_{h}^{q}=D^{q} \hat{x}_{q, h^{*}}$

Si $u_{h}=\left(u_{h}^{\alpha}\right)$ est une suite sur $z^{n}$, nous poserons :

(2-6) $\quad\left(T_{\gamma} u_{h}\right)^{\alpha}=u_{h}^{\alpha-\gamma} ; \nabla_{h}^{q}=h^{-q} \nabla^{q}=h^{-q} \sum_{k \leqslant q}(-1)|k| \quad c_{q}^{k} T_{(k-q)}$

EXPRESSIONS EXPLICITES DES FONCTIONS $x_{K+1} \cdot$

Considérons le cas où $h=1, n=1$, à partír duquel on obtient aisément le cas général. Puisque $X=\nabla Y$, où $Y$ est la distribution de Heaviside, on en déduit que sur chaque intervalle $(-K-1+k,-K+k)$, la fonction $x_{K+1}$ est un polynôme $\beta_{K}^{k}(x)$ :

$(2-7)\left\{\begin{array}{l}X_{K+1}(x)=\sum_{k=0}^{K} \beta_{K}^{k}(x) x^{-K+k}(x) \text { où } \\ \beta_{K}^{k}(x)=\frac{1}{K !} \sum_{q=0}^{k}(-1)^{q} C_{K+1}^{q}(x+K+1-q)^{K} .\end{array}\right.$

Il sera commode d'introduíre les polynômes :

$(2-8) \quad \psi_{K}^{k}(x)=\delta(K-k)^{*} B_{K}^{k}(x)=\sum_{q=0}^{K} a_{K}(k, q) \frac{1}{q !}(x+1)^{q}$ 
où

$$
a_{K}(k, q)=\left\{\begin{array}{cl}
\frac{1}{(K-q) !} & \sum_{p=0}^{k}(-1)^{p} c_{K+1}^{p}(k-p)^{K-q} \text { si } 0 \leqslant k \leqslant k . \\
0 \text { si } & k \leqslant-1 \text { et si } k \geqslant k+1
\end{array}\right.
$$

On remarque alors que :

$(2-10) \quad a_{K}(k, q)=D^{q} x_{K+1}(-K-1+k)=D^{q} \psi_{K}^{k}(-1)$

et que $\sum_{k=0}^{K} \psi_{K}^{k}(x)=1$ puisque les $x_{K+1}^{\alpha}$ forment une partition de 1'unité.

On utilisera, parmi d'autres, les propriétés suivantes des coefficients $a_{K}(k, q):$

$$
\text { q) : } \begin{aligned}
\text { i) } \sum_{k=0}^{K} a_{K}(k, q)=\left\{\begin{array}{lll}
0 & \text { si } q=1, \ldots, k \\
1 & \text { si } q=0 .
\end{array}\right. \\
\text { ii) } a_{k}(0, q)=\left\{\begin{array}{lll}
0 & \text { si } q \leqslant K-1 \\
1 & \text { si } q=K
\end{array}\right. \\
\text { iii) } a_{K}(k, k)=(-1)|k| c_{K}^{k} \\
\text { iv) } a_{K}(k, q)=\sum_{j=0}^{p}(-1)|j| c_{p}^{j} a_{K-p}(k-j, q-p) \\
\text { v) } a_{K}(k, q)=(-1)|q| a_{K}(K+1-k, q)
\end{aligned}
$$

LES QUOTIENTS DIFFERENTIELS $\mathrm{Q}_{\mathrm{K}}^{\mathrm{q}}$.

Nous poserons, si $K=\left(K_{1}, \ldots, K_{n}\right)$ est un multientier

(2-12) $Q_{K, h}^{q}=h^{-q} \sum_{k=0}^{K} a_{K}(k, q) \delta(-k+k) *$

où

$$
\begin{aligned}
a_{K}(k, q) & =\prod_{i=1}^{n} a_{K_{i}}\left(k_{i}, q_{i}\right) \\
\text { Si } u_{h} & =\left(u_{h}^{\alpha}\right) \text { est une suite, nous poserons : } \\
\left(Q_{K}^{q} u_{h}\right)^{\alpha} & =\sum_{k=0}^{m} a_{K}(k, q) u_{h}^{\alpha+K-k .}
\end{aligned}
$$

Nous verrons que ces opérateurs sont des quotients différentiels. On déduit de (2-11) que : 
$(2-13)$

$$
\begin{aligned}
\text { i) } Q_{K}^{q} & =Q_{K-k}^{q-k} \nabla^{k}=\nabla^{k} Q_{K-k}^{q-k} \\
\text { ii) } Q_{K}^{K} & =\nabla^{K} \\
\text { iii) } Q_{1}^{0} & =1, Q_{1}^{1}=\nabla \quad(s i n=1)
\end{aligned}
$$

LES FONCTIONS $\zeta_{\mathrm{K}}(\mathbf{x})$.

LEMME 2-1.

Il existe une fonction $\zeta_{\mathrm{K}}(\mathrm{x})=\mathrm{x}(\mathrm{x}) \sum_{\mathrm{q}=0}^{\mathrm{K}} \zeta_{\mathrm{K}}^{\mathrm{q}} \frac{1}{\mathrm{q} !}(\mathrm{x}+1)^{\mathrm{q}}$ vérifiant :

$(2-14) h<x_{K+1, h}^{\alpha}, \zeta_{K, h}^{\beta}>=\left\{\begin{array}{lll}0 & \text { si } & \alpha \neq \beta \\ 1 & \text { si } \alpha=\beta\end{array} ; \int \zeta_{K}(x) d x=1\right.$

Les coefficients $\zeta_{\mathrm{K}}^{\mathrm{q}}$ sont dêterminés par les $\mathrm{K}$ relations :

$$
\sum_{q=0}^{K} \zeta_{K}^{q}\left(\sum_{p=0}^{K} \frac{a_{K}(k, p)}{q ! p !(p+q+1)}\right)=\left\{\begin{array}{lll}
0 & \text { si } & k=1, \ldots, k-1 \\
1 & \text { si } & k=k
\end{array}\right.
$$

On remarque tout d'abord que :

$$
\left.h<x_{K, h}^{\alpha}, \quad \zeta_{K, h}^{\beta}\right\rangle=\left\langle x_{K}^{\alpha-\beta}, \zeta_{K}>\right.
$$

Il suffit donc de vérifier que :

$$
\int_{-1}^{0} \zeta_{K}(x) \psi_{K}^{k}(x) d x=\sum_{q=0}^{K} \zeta_{K}^{q} \int_{-1}^{0} \frac{(x+1)^{q}}{q !} \psi_{K}^{k}(x) d x=\left\{\begin{array}{lll}
0 & \text { si } & k \leqslant K-1 \\
1 & \text { si } & k=K
\end{array}\right.
$$

Puisque les polynômes $(x+1)^{q}$ et $\psi_{K}^{k}(x)$ sont linéairement indépendants, la matrice des coefficients $\int_{-1}^{0}(x+1)^{q} \psi_{K}^{k}(x)$ dx est inversible et les coefficients $\zeta_{K}^{q}$ sont déterminés de façon unique.

On déduit alors $(2-15)$ de $(2-8)$ et puisque la somme des polynômes $\Psi_{K}^{k}(x)$ est égale à 1 , on en déduit que $\zeta_{K}$ est une fonction de masse totale égale à 1 . REMARQUE 2-1.

On peut plus généralement montrer l'existence d'une fonction $\zeta_{\mathrm{K}}$ indéfiniment différentiable à support dans $(-1,0)$ vérifiant les propriétés $(2-14)$. Ce lemme exprime alors que les fonctions $\chi_{K}^{\alpha}$ sont topologiquement libres dans l'espace $D^{\prime}$ des distributions. 
2.2. APPROXIMATION DES ESPACES DE SOBOLEV $\mathrm{w}^{\mathrm{m}, \mathrm{P}}$

Nous allons construire des approximations des espaces de Sobolev $W^{m \cdot P}=W^{m, P}\left(R^{n}\right)$. Ce sont des espaces de Banach pour les normes (2-16) ||$u||_{m, P}=\left(\sum_{|q| \leqslant m}\left|D^{q} u\right|_{P}^{P}\right)^{1 / p}:|u|_{P}=\left(\int|u(x)|^{P} d x\right)^{1 / P}$.

Nous désignerons par $W_{h}^{m}, P$ l'espace des suites de puissance $P^{\text {ième }}$ sommables sur $\mathrm{z}^{\mathrm{n}}$ muni de la norme :

$(2-17)\left\{\begin{array}{l}\left.||_{h}\right|_{h, m, P}=\left(\sum_{|q| \leqslant m}\left|\nabla_{h}^{q} u_{h}\right|_{P}^{p}\right)^{1 / p} \\ \left|u_{h}\right|_{h, P}=\sqrt[P]{h} / \sqrt{h} \underset{\alpha}{\left(\sum_{\alpha}\left|u_{h}^{\alpha}\right|^{p}\right)^{1 / P}}\end{array}\right.$

Considérons les opérateurs de prolongement et de restrictions suivants : Si $\mathrm{K}$ est un multientier $k=\left(K_{1}, \ldots, K_{n}\right)$, nous poserons :

$(2-18)\left\{\begin{array}{l}\mathrm{P}_{\mathrm{h}}^{\mathrm{K}} u_{\mathrm{h}}=\sqrt{\mathrm{h}} \sum_{\alpha} u_{\mathrm{h}}^{\alpha} \mathrm{x}_{\mathrm{K}+(1), \mathrm{h}}^{\alpha} \\ \mathrm{r}_{\mathrm{h}}^{* \mathrm{~K}} \phi=\left(\sqrt{\mathrm{h}} \int \phi(\mathrm{x}) \mathrm{x}_{\mathrm{K}+(1), \mathrm{h}}^{\alpha}(\mathrm{x}) \mathrm{dx}\right)_{\alpha} \\ \mathrm{r}_{\mathrm{h}}^{\mathrm{K}} \phi=\left(\sqrt{\mathrm{h}} \int \phi(\mathrm{x}) \zeta_{\mathrm{K}, \mathrm{h}}^{\alpha}(\mathrm{x}) \mathrm{dx}\right)_{\alpha}\end{array}\right.$

Désignons par $r_{h}$ l'une des restrictions $r_{h}^{* L}, r_{h}^{K}$.

Considérons alors les approximations :

$(2-19) \quad A_{h}^{K}\left(w^{m, P}\right)=\left(w^{m, P}, w_{h}^{m, P}, p_{h}^{K}, r_{h}\right)$

THEOREME 2-1.

Si $K \geqslant(m)$ et $L \geqslant 0$, les opérateurs $p_{h}^{K}, r_{h}^{* L}$ et $r_{h}^{K}$ vêrifient les propriétés suivantes.

Les prolongements $p_{h}^{K}$ s'explicitent ainsi :

$(2-20) \quad p_{h}^{K} u_{h}=h^{+1 / 2} \sum_{\alpha}\left(\sum_{q \leqslant K}\left(Q_{K}^{q} u_{h}\right)^{\alpha} \frac{1}{q !}\left(\frac{x}{h}-\alpha+1\right)^{q}\right) x_{h}^{\alpha}$

- Les opérateurs de dérivation et les quotients diffêrentiels commutent avec ces opérateurs : 


$$
\left\{\begin{array}{l}
\text { i) } D^{q} p_{h}^{K} u_{h}=p_{h}^{K-q}\left(\nabla_{h}^{q} u_{h}\right) ; \nabla_{h}^{q} r_{h} \phi=r_{h}\left(\nabla_{h}^{q} \phi\right) \\
\text { ii) } r_{h}^{* L} D^{q} p_{h}^{K} u_{h}=h^{-q} T_{+L} Q_{K+L+(1)}^{q} u_{h}
\end{array}\right.
$$

Les prolongements $p_{h}^{K}$ sont des isomorphisines et de pius :

(2-22) $\mathrm{r}_{\mathrm{h}}^{\mathrm{K}} \mathrm{p}_{\mathrm{h}}^{\mathrm{K}} \mathrm{u}_{\mathrm{h}}=u_{\mathrm{h}} ; \mathrm{p}_{\mathrm{h}}^{\mathrm{K}} \mathrm{r}_{\mathrm{h}}^{\mathrm{K}}$ est un projecteur de $\mathrm{w}^{\mathrm{m}, \mathrm{P}}$

Enóin, les approximations de $w^{\mathrm{m}, P}$ sont stabies et convergentes :

(2-23) $\left\{\begin{array}{l}\text { 1) }\left\|p_{h}^{K} u_{h}\right\|_{m, P} \leqslant\left\|u_{h}\right\|_{h, m, P} \leqslant m\left\|p_{h}^{K} u_{h}\right\|_{m, P} \\ \text { ii) }\left\|r_{h} \phi\right\|_{h, m, P} \leqslant m|| \phi \|_{m, P}\end{array}\right.$

(2-24) $\left\|\phi-p_{h}^{K} r_{h} \phi\right\|_{P} \leqslant c\left(\sum_{|q| \leqslant m} \omega_{P}^{P}\left(D^{q} \phi, h\right)^{1 / p}\right.$

De plus les normes de $1^{\mathrm{P}}$ vérifient:

$\left.(2-25) \quad\left|u_{h}\right|\right|_{h, m, P} \leqslant c\left(\sum_{|q| \leqslant m} h^{-P q}\right)^{1 / p}\left|u_{h}\right|_{h, P}$.

REMARQUES 2-2.

Si $K=L$, les approximations $\left(w^{m, P}, w_{h}^{m, P}, p_{h}^{K}, r_{h}^{* K}\right.$ ) sont autoadjointes, stables et convergentes.

$D^{\prime}$ après $(2-13) \mathrm{iii)}$, on voit que pour $K=(1)$,

$$
p_{h}^{(1)} u_{h}=h^{-\frac{1}{2}} \sum_{\alpha}\left(u_{h}^{\alpha}+\sum_{0 \neq q \leqslant(1)}\left(\nabla^{q} u_{h}\right)^{\alpha}\left(\frac{x}{h}-\alpha+1\right)^{q}\right) \theta_{h}^{\alpha}
$$

Ce prolongement a été obtenu sous cette forme par 0.A. LADYJENSKAIA [1] . On trouvera dans J. CEA [2] les prolongements qui ont la propriété supplémentaire d'interpolation :

$$
D^{q} p_{h} u_{h}((\alpha-1) h)=\left(\nabla_{h}^{q} u_{h}\right)^{\alpha} \quad \text { pour } q \leqslant K
$$

On voit d'après $(2-21) i i)$ que les opérateurs $Q_{K}^{q}$ sont des restrictions des opérateurs de dérivation $D^{q}$. En particulier, les opérateurs $Q_{K}^{0}$, restrictions de l'identité, sont des opérateurs de "1issage" (ou de régularisation discrète), puisque, d'après $(2-11) i),\left(Q_{K}^{\circ} u_{h}\right)^{\alpha}$ est un barycentre des éléments $u_{h}^{\alpha+k}(0 \leqslant k \leqslant k)$. 
On déduit enfin de l'inégalité (2-24) que :

$$
\left\|\phi-p_{h}^{K} r_{h}^{* L} \phi\right\|_{m, P} \leqslant c h\|\phi\|_{m+1, P}
$$

Les fonctions $p_{h}^{K} u_{h}$ sont des fonctions dont les restrictions a chaque intervalle $(\alpha h,(\alpha+1) h$ ) (dans le cas $n=1$ ) sont des polynômes de degré $K$. Ce sont des exemples de "Spline functions" introduites par I.J. SCHOENBERG [1] et étudiées par de nombreux auteurs à propos du problème d'interpolation optimale (cf. aussi J.P. AUBIN [7]). Le théorème 2-1 énonce donc (dans le cas d'un réseau régulier) les propriétés des dérivées des "spline functions" et précise les propriétés d'approximation de ces fonctions.

On trouvera dans J.P. AUBIN [3] une évaluation plus précise des erreurs de troncature : $i 1$ existe des restrictions $s_{h}^{K}$ telles que :

$$
\left\|D^{q} p_{h}^{K} s_{h}^{K} \phi-D^{q} \phi\right\|_{P} \leqslant c h^{K+(1)-q}\left\|D^{K+(1)} \phi\right\|_{P} \text { si } q \leqslant K+(1)
$$

On peut obtenir à partir de ces approximations d'autres approximations de $W^{\mathrm{m}, P}$ en utilisant la proposition 1-7 du $n^{\circ} 1-10$ du $\$ 1$ du chapitre $I$ en prenant pour opérateurs $P_{h}$ et $R_{h}$, des opérateurs de convolution par $\sigma_{h}(x)=\frac{1}{h} \sigma\left(\frac{x}{h}\right)$, où $\sigma$ est une fonction positive à support compact de masse totale égale à 1 , soit des opérateurs de multiplication par des fonctions $\gamma_{h}$ à support compact, convergeant vers 1 dans $w^{m, \infty}$, soit des difféomorphismes réciproques, ce quí donne des approximations sur des réseaux irréguliers. On peut naturellement varier les exemples à 1 'infini.

DEMONSTRATION DU THEOREME $2-1$.

a) EXPRESSION EXPLICITE DE $p_{h}^{K} u_{h}$.

Faisons $h=1$. On déduit alors cette identité de la définition des polynômes $\psi_{\mathrm{K}}^{\mathrm{k}}(\mathrm{x})$ et de (2-8). Puisque :

$(2-27) \quad x_{K+1}^{\alpha}=\sum_{k \leqslant K} \delta(-k+k+\alpha) *\left(\psi_{K}^{k}(x) x(x)\right)$

il en résulte que :

$$
\begin{aligned}
p^{K} u=\sum_{\alpha} u^{\alpha} x_{K+1}^{\alpha} & =\sum_{\alpha} u^{\alpha} \sum_{k \leqslant K} \delta(-K+k+\alpha) *\left(\psi_{K}^{k} x\right)= \\
& =\sum_{\alpha}^{\cdot}\left(\sum_{k \leqslant K} u^{\alpha+K-k} \psi_{K}^{k}(x-\alpha)\right) x^{\alpha}= \\
& =\sum_{\alpha} \sum_{k \leqslant K} \sum_{q \leqslant K} a_{K}(k, q) u^{\alpha+K-k} \frac{1}{q !}(x+1-\alpha)^{q} x^{\alpha}
\end{aligned}
$$


b) PROPRIETES DE COMMUTATIVITE.

On remarque tout d'abord que les opérateurs de translation commuttent avec les opérateurs de prolongement et de restriction :

$$
\begin{aligned}
\tau_{h \beta} p_{h}^{K} u_{h} & =\sum_{\alpha} u_{h}^{\alpha} x_{K+(1), h}^{\alpha+\beta}=\sum_{\alpha} u_{h}^{\alpha-\beta} x_{K+(1), h}^{\alpha}=p_{h}^{K}\left(\tau_{\beta} u_{h}\right) \\
\text { Si } r_{h} \phi & =\left(\int \phi s_{h}^{\alpha} d x\right)_{\alpha} ; s_{h}^{\alpha}=\delta(\alpha h) * s_{h}: \\
\left(\tau_{B} r_{h} \phi\right)^{\alpha} & =\left(\int \phi s_{h}^{\alpha-\beta} d x\right)=\left(\int \tau_{\beta h} \phi s_{h}^{\alpha} d x\right)=\left(r_{h} \tau_{\beta h} \phi\right)^{\alpha}
\end{aligned}
$$

Il en résulte alors que les quotients différentiels commuttent :

$$
\nabla_{h}^{q} p_{h}^{K} u_{h}=p_{h}^{K} \nabla_{h}^{q} u_{h} ; \quad \nabla_{h}^{q} r_{h} \phi=r_{h} \nabla_{h}^{q} \phi .
$$

$D^{\prime}$ autre part, puisque d'après (2-5), $D^{q} x_{K}=\nabla^{q} x_{K-q}$, on en déduit (2-21) 1 ). Montrons maintenant que $r_{h}^{* L} p_{h}^{K}=Q_{K+L+1}^{0} \tau_{L}$.

$$
\text { En effet, }\left(x_{h}^{* L} p_{h}^{K} u_{h}\right)^{\alpha}=h \sum_{B}\left[\int x_{K+(1), h}^{\beta}(x) x_{L+(1), h}^{\alpha}(x) d x\right] u_{h}^{\beta}
$$

Mais puisque $\int \sigma \phi=\sigma * \grave{\phi}(0)$, où $\grave{\phi}(x)=\phi(-x)$, on en déduit, d'après $(2-10)$, que :

$h \int x_{K+(1), h}^{\beta}(x) x_{L+(1), h}^{\alpha}(x) d x=x_{K+L+(2)}^{L+1+\beta-\alpha}(0)=a_{K+L+(1)}(K+(1)+\alpha-\beta, 0)$

Donc :

$$
\begin{aligned}
\left(x_{h}^{* L} p_{h}^{K} u_{h}\right)^{\alpha} & =\sum_{B} a_{K+L+(1)}(K+(1)+\alpha-\beta, 0) u_{h}^{B}= \\
& =\sum_{k \in K} a_{K+L+(1)}(k, 0) u_{h}^{K+L+(1)+\alpha-L-k}=\left(\tau_{L} Q_{K+L+(1)}^{0} u_{h}\right)^{\alpha}
\end{aligned}
$$

On en déduit que :

$$
\left.r_{h}^{* L} D^{q} p_{h}^{K}=r_{h}^{* L} p_{h}^{K-q} \nabla_{h}^{q}=Q_{K+L+(1)-q}^{0}\left(\nabla_{h}^{q}\right)=h^{-q} Q_{K+L+(1)}^{q} \text { d'après (2-13) } 1\right) \text {. }
$$

Nous allons montrer que $r_{h}^{K}$ est un inverse à gauche de $p_{h}^{K}$, ce qui montrera en particulier que $p_{h}^{K}$ est un isomorphisme et que $p_{h}^{K} r_{h}^{K}$ est un projecteur continu de $W^{\mathrm{m}, P}$.

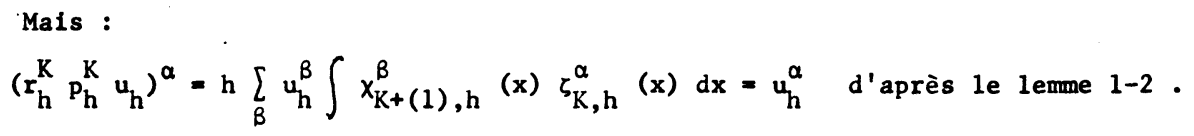


89

c) STABILITE DES PROLONGEMENTS ET DES RESTRICTIONS.

Remarquons tout d'abord que

$$
p_{h}^{K} u_{h}=x_{K, h} * p_{h}^{0} u_{h} \quad ; \quad p_{h}^{0} u_{h}=h^{1 / 2} \sum_{\alpha} u_{h}^{\alpha} x_{h}^{\alpha}(x)
$$

Montrons que :

(2-30) $\quad\left|p_{h}^{0} u_{h}\right|_{P} \leqslant\left|u_{h}\right|_{h, P}$

$$
\begin{aligned}
& \text { En effet : } \\
& \int\left|p_{h} u_{h}\right|^{P} d x \leqslant h^{P / 2} \int \sum_{\alpha}\left|u_{h}^{\alpha}\right|^{P}\left|x_{h}^{\alpha}(x)\right|^{P} d x \leqslant h^{1-P / 2} \sum_{\alpha}\left|u_{h}^{\alpha}\right|^{P}
\end{aligned}
$$

Donc, puisque $\int x_{K, h}(x) d x=1$, il en résulte que pour tout $|q| \leqslant m$ :

(2-31) $\quad\left|D^{q} p_{h}^{K} u_{h}\right|_{P}=\left|x_{K-q, h} * p_{h}^{o}\left(\nabla_{h}^{q} u_{h}\right)\right|_{P} \leqslant\left|p_{h}^{0} \nabla_{h}^{q} u_{h}\right|_{P}$

il en résulte que $\quad\left\|p_{h}^{K} u_{h}\right\|_{m, P} \leqslant\left\|u_{h}\right\|_{h, m, P}$.

De même :

(2-32) $\quad\left|r_{h} \phi\right|_{h, P} \leqslant m|\phi|_{P}$

En effet, soit $s_{h}^{\alpha}(x) \quad l^{\prime}$ une des fonctions $x_{L+(1), h}^{\alpha}(x), \zeta_{K, h}^{\alpha}(x)$, qui sont à support compact et de masse totale égale à 1 .

Soit $\mathrm{m}_{1}$ la borne supérieure de $\sum_{\alpha} \mathrm{s}(\mathrm{x}-\alpha)$ et $\mathrm{m}_{2}$ la norme de $\mathrm{s}(\mathrm{x})$ dans ${ }_{L}^{P}, m_{P}=m_{1} m_{2}^{P-1}$. On déduit (2-32) de 1'inégalité :

$$
\begin{aligned}
& \sum_{\alpha} \int\left|\phi s_{h}^{\alpha}(x) d x\right|^{P} \leqslant \sum_{\alpha}\left(\int|\phi(x)|^{P}\left|s_{h}^{\alpha}(x)\right| d x\right)\left(\int\left|s_{h}^{\alpha}(x)\right| d x\right)^{P / P^{\prime}} \leqslant \\
& \leqslant m_{P} h^{-1} \int|\phi(x)|^{P} d x
\end{aligned}
$$

Il en résulte donc que :

$$
\left|\nabla_{h}^{q} r_{h} \phi\right|_{h, P} \leqslant\left|r_{h}\left(\nabla_{h}^{q} \phi\right)\right|_{P} \leqslant m_{P}\left|\nabla_{h}^{q} \phi\right|_{P} \leqslant m_{P}\left|D^{q} \phi\right|_{P}
$$

puisque $\nabla_{h}^{q} \phi=x_{q, h} * D^{q} \phi$.

De plus, en prenant $r_{h}=r_{h}^{K-q}$, 11. vient :

$$
\left|\nabla_{h}^{q} u_{h}\right|_{h, p}=\left|\nabla_{h}^{q} r_{h}^{K-q} p_{h}^{K-q} u_{h}\right|_{h}=\left|r_{h}^{K-q} D^{q} p_{h}^{K} u_{h}\right| \leqslant m_{P}\left|D^{q} p_{h}^{K} u_{h}\right|_{P}
$$


ce qui montre que :

(2-33) $\quad\left\|u_{h}\right\|_{h, m, P} \leqslant m|| p_{h}^{K} u_{h} \|_{m, P} ; m$ indépendante de $h$.

D'autre part, (2-25) est évident puisque :

(2-34) $\quad\left|\nabla_{h}^{q} u_{h}\right|_{P} \leqslant h^{-q}{ }_{h}^{q}\left|\nabla_{h}^{q} u_{h}\right|_{P} \leqslant c h^{-q}\left|u_{h}\right|$

d) CONVERGENCE DES APPROXIMATIONS.

On remarque que :

$(2-35)\left\{\begin{aligned} \mid D^{q}\left(\phi-p_{h}^{K}\right. & \left.r_{h} \phi\right)\left.\right|_{P} \leqslant\left|D^{q} \phi-p_{h}^{K-q} r_{h} D^{q} \phi\right|_{P}+ \\ + & \left|p_{h}^{K-q} r_{h}\left(D^{q} \phi-x_{q, h} * D^{q} \phi\right)\right|_{P}\end{aligned}\right.$

Il suffit alors de montrer que :

(2-36) $\quad\left|\phi-p_{h}^{K} r_{h} \phi\right|_{P} \leqslant c \omega_{P},(\phi, h)$

(2-37) $\quad\left|\phi-x_{q, h} * \phi\right|_{p} \leqslant c \omega_{p}(\phi, h)$

Montrons tout d'abord (2-36) . Si $s_{h}^{\alpha}=\chi_{L, h}^{\alpha}$ ou $\zeta_{K, h}^{\alpha}$ :

(2-38) $\phi(x)=\sum_{\alpha}\left(\int \phi(x) s_{h}^{\alpha}(y) d y\right) h x_{K+(1), h}^{\alpha}$

On en déduit que :

(2-39) $\phi(x)-p_{h} r_{h} \phi(x)=x_{K, h} * \tau_{h}(\phi)$

où

(2-40) $\tau_{h} \phi=\sum_{\alpha}\left(\int(\phi(x)-\phi(y)) s_{h}^{\alpha}(y) d y\right) h x_{h}^{\alpha}$

Il suffit de montrer que pour toute $\phi$ continue :

(2-41) $\left|\tau_{h} \phi\right|_{P} \leqslant c \omega_{P}(\phi, h)$.

Mais

$\int d x\left|\tau_{h} \phi\right|^{P} \leqslant \sum_{\alpha}\left|\int\right| \phi(x)-\phi(y)|| s_{h}^{\alpha}(y)|d y|^{P} h x_{h}^{\alpha} d x \leqslant$

$\leqslant m \sum_{\alpha} \int d x h x_{h}^{\alpha} \underset{|x-y| \leqslant c h}{\sup _{\mid}}|\phi(x)-\phi(y)|^{P}=m \omega_{p}^{P}(\phi, c h)$. 
Démontrons maintenant $(2-37)$, en posant $\sigma=\chi_{q}$. Si $\phi$ est continue : $\left|\sigma_{h} * \phi-\phi\right|_{P}^{P} \leqslant h^{-P} \int d x\left|\int_{\omega_{h}} \sigma\left(\frac{x}{h}\right)(\phi(t-x)-\phi(x)) d t\right|^{P} \leqslant$ $\leqslant \sup _{t \in \omega_{h}} \int|\phi(t-x)-\phi(x)|^{P} d x=\omega_{P}^{P}\left(\phi, \omega_{h}\right) \leqslant c \omega_{P}^{P}(\phi, h)$

On en déduit (2-37) puisque les fonctions continues sont données dans $L^{P}$. Puisque $\left\|p_{h}^{K} u_{h}\right\|_{m, P}=\left\|u_{h}\right\|_{h, m, P} \leqslant m\left\|p_{h}^{K} u_{h}\right\|_{m, P}$, nous en déduisons le

COROLlaIre 2-1. (Inégalités de Sobolev discrètes).

Si $\frac{1}{q}=\frac{1}{p}-\frac{m}{n}>0$, alors

(2-42) $\left|u_{h}\right|_{h, q} \leqslant c|| u_{h}||_{h, m, P}$, où $c$ est indépendante de $h$.

Si $m-\frac{p}{n}=k+\theta, k$ entier $\geqslant 0,0<\theta \leqslant 1$, pour tout ensemble fini $R_{h}$ de $\boldsymbol{z}^{\mathrm{n}}$, nous obtenons :

(2-43) $\sup _{\alpha \in R_{h}} \sup _{|r| \leqslant k}\left|\nabla_{h}^{r} u_{h}^{\alpha}\right| \leqslant\left. c|| u_{h}\right|_{h, m, p}$ où $c$ est indépẹndante de $h$.

Ces inégalités résultent des inégalités de Sobolev sur les fonctions :

$|\phi|_{q} \leqslant c|| \phi||_{m, P}$ si $\frac{1}{q}=\frac{1}{p}-\frac{m}{n}>0$ et $\sup _{K} \sup _{|r| \leqslant k}\left|D^{r} \phi\right| \leqslant c|| \phi||_{m, P}$

si $m-P / n=k+\theta$.

REMARQUE 2-3.

On peut donner des démonstrations directes de ces inégalités, soit en adaptant la démonstration initiale de Sobolev (cf. SOBOLEv [3] [2] ), soit en adaptant ce1le de GAGLIARDO [1] .

On démontre de la même manière des inégalités discrètes analogues aux inégalités de J.L. LIONS [4], sur les fonctions à valeurs vectorielles, généralisant les inégalités de GAGLIARDO - NIRENBERG . 
2-3. APPROXIMATION DES ESPACES DE DISTRIBUTIONS.

Soitt $X$ un espace de distributions. Nous désignerons par $X^{m}$ l'espace des distributions de $X$ dont toutes les dérivées jusqu'à l'ordre m appartiennent à $X$. Nous munirons l'espace $X^{\mathrm{m}}$ de la topologie la moins fine rendant continues les dérivations $D^{q}$. On peut prendre par exemple pour $X$ les espaces de fonctions continues du $\$ 1$.

Donnons-nous des approximations discrètes de l'espace $X$ :

(2-44) $\quad A_{h}(X)=\left(X, X_{h}, p_{h}, r_{h}\right)=\left(X, \sigma_{h}, s_{h}\right)$

où $\sigma_{h}=\left(\sigma_{h}^{\alpha}\right)_{\alpha}$ est une suite de $X, S_{h}=\left(s_{h}^{\alpha}\right)_{\alpha}$ une suite de $X^{\prime}$. On démontre de la même manière qu'au numéro précédent le :

THEOREME 2-2.

Supposons que :

$(2-45) \quad \sigma_{h}^{\alpha}=\delta(\alpha h) * \sigma_{h} ; s_{h}^{\alpha}=\delta(\alpha h) * s_{h}$

Si les approximations $A_{h}(x)$ sont stables et convergentes, les approximations de $x^{\mathrm{m}}$ :

(2-46) $\quad A_{h}\left(x^{m}\right)=\left(x^{m}, x_{h}, p_{h}^{K}, r_{h}\right) ; p_{h}^{K}=x_{h}^{K} * p_{h}$

sont également stables et convergentes si $k \geqslant(m)$.

Plus généralement, soit $G(t)$ un groupe continu d'opérateurs de $L(X, X)$, A son générateur infinitésimal, $D(A)$ son domaine.

Si $\theta$ est une fonction et $\phi$ un élément de $X$, nous poserons :

$$
G(\theta) \phi=\int_{-\infty}^{+\infty} G(t) \theta(t) \phi d t
$$

THEOREME 2-3.

Supposons que :

(2-47) $\quad \sigma_{h}^{\alpha}=G(\alpha h) \quad \sigma_{h} ; s_{h}^{\alpha}=G(\alpha h) s_{h}$

Si les approximations $A_{h}(X)$ sont stables et convergentes, les approximations discrètes du domaine $\mathrm{D}\left(\mathrm{A}^{\mathrm{m}}\right)$ de $\mathrm{A}^{\mathrm{m}}$

$$
A_{h}\left(D\left(A^{m}\right)\right)=\left(D\left(A^{m}\right), X_{h}, p_{h}^{K}, r_{h}\right) ; p_{h}^{K}=G\left(X_{h}^{K}\right) p_{h}
$$


sont également stables et convergentes si $\mathrm{K} \geqslant \mathrm{m}$.

On déduit ce théorème de l'identité :

$(2-48)$ A G $\left(x_{h}\right)=G(-h)-G(0)$

La démonstration est alors analogue à celle du théorème 2-1.

$\mathrm{Si} X$ est un espace de fonctions continues ou un espace $L^{P}$ et $s i$ $Y$ est $1 \mathrm{a}$ distribution de Heaviside, les fonctions :

$$
\theta_{h}^{\alpha}=G(\alpha h) \theta_{h} \quad ; \quad \theta_{h}=[G(0)-G(h)] Y
$$

forment une partition de l'unité. On peut alors construire. à l'aide des théorèmes du $\$ 1$, des approximations de $X$ vérifiant $(2-45)$ stables et convergentes.

\$3. APPROXIMATION DES ESPACES DE SOBOLEV SUR UN OUVERT $\Omega$.

Nous déduisons du théorème 2-1 du $\$ 2$ la construction d'approximations et d'approximations partielles des espaces de Sobolev $\mathrm{W}_{0}^{\mathrm{m}, \mathrm{P}}(\Omega) \quad\left(\mathrm{n}^{\circ} 3-2\right)$ et $\mathrm{w}^{\mathrm{m}, \mathrm{P}}(\Omega)$ $\left(n^{\circ} 3-3\right)$ sur un ouvert $\Omega$. Il faut pour cela définir des réseaux associés à un ouvert $\Omega\left(n^{\circ} 3-1\right)$. Au $n^{\circ} 3-4$, on construit des approximations de sous-ensembles convexes des espaces de Sobolev, en vue des applications aux inéquations variationnelles.

On suppose connu le $\S 2$ de ce chapitre.

Rappelons que $\mathfrak{W}^{\mathrm{m}, \mathrm{P}}(\Omega)$ est l'espace des fonctions u de $L^{\mathrm{P}}(\Omega)$ dont les dérivées jusqu'à l'ordre $m$ appartiennent encore à $L^{P}(\Omega)$, que $W^{q, P}(\Omega)$ est $I^{\prime}$ espace des fonctions $u$ de $L^{P}(\Omega)$ dont la dérivée d'ordre $q=\left(q_{1}, \ldots, q_{n}\right)$ appartient à $L^{P}(\Omega) ; W^{m, P}(\Omega)$ est $l^{\prime}$ intersection des espaces $W^{q}, P(\Omega)$ pour $|q| \leqslant m$. On désigne par $w_{0}^{\mathrm{m}, P}(\Omega) \quad\left(\right.$ resp. $W_{0}^{q, P}(\Omega)$ ) l'adhérence dans $w^{\mathrm{m}, \mathrm{P}}(\Omega)$ (resp. $W^{q, P}(\Omega)$ ) des fonctions à support compact dans $\Omega$.

3-1. LES RESEAUX $R_{h}^{K}(\Omega)$.

Solent $\Omega$ un ouvert de $\mathbb{R}^{n}$, de frontière $r, h=\left(h_{1}, \ldots, h_{n}\right)$ le pas du maillage, $K$ un multientier. Nous allons associer à $\Omega, h, K$ des réseaux, c'està-dire des sous-ensembles de $z^{\mathfrak{n}}$. Ráppelons que nous avons posé : (\$2,(2-2) )

$(3-1)\left\{\begin{aligned} \omega_{k, h}^{\alpha} & \left.=\prod_{i=1}^{n}\left[\left(\alpha_{i}-k_{i}-1\right) h_{i}, \alpha_{i} h_{1}\right)\right]=\text { support de } x_{K+(1), h}^{\alpha} \\ & =\bigcup_{k \leqslant K} \tau_{-k} \omega_{h}^{\alpha}=\bigcup_{k \leqslant K} \omega_{h}^{\alpha-k} ; \quad\left(\omega_{h}^{\alpha}=\omega_{0, h}^{\alpha}\right)\end{aligned}\right.$ 
Si $E$ est un sous-ensemble de $R^{n}$, nous poserons :

(3-2) $\quad R_{h}^{K}(E)=\left\{\alpha \varepsilon z^{n}\right.$ tels que $\left.\omega_{K, h}^{\alpha} \cap E \neq \Phi\right\}$

En particulier, il est clair que d'après (3-1):

(3-3) $R_{h}^{K}(E)=\bigcup_{k \in K} \tau_{k} R_{h}$ (E)

Nous considérerons les réseaux $R_{h}^{K}(\Omega), R_{h}^{L}(\Gamma)$, etc...

Nous poserons :

(3-4) $\quad R_{\mathrm{h}}^{\mathrm{K}}(\Omega ; \Gamma)=R_{\mathrm{h}}^{\mathrm{K}}(\Omega)-R_{\mathrm{h}}^{\mathrm{K}}(\Gamma)$.

LEMME 3-1.

Soit $u_{h}=\left(u_{h}^{\alpha}\right)$ une suite diépinie sur $R_{h}^{K}(\Omega)$. Les quotients dijó́rentiels $\mathrm{Q}_{\mathrm{K}}^{\mathrm{q}} \mathrm{u}_{\mathrm{h}} \quad\left(c \hat{b} . \$ 2, n^{0} 2-1\right)$ sont définis sur $R_{h}(\Omega)($ pour $q \leqslant \mathrm{~K})$.

En effet, si $a \in R_{h}(\Omega)$ :

(3-5) $\quad\left(Q_{K}^{q} u_{h}\right)^{\alpha}=\sum_{k \leqslant K} a_{K}(K-k, q) u_{h}^{\alpha+k}$

et les indices $\alpha+\mathrm{k}$ appartiennent à $R_{h}^{\mathrm{K}}(\Omega)$. En particulier, les quotients $\nabla^{\mathrm{K}}=\mathrm{O}_{\mathrm{K}}^{\mathrm{K}}$ sont définis sur $R_{\mathrm{h}}(\Omega)$.

THEOREME 3-1.

Si $\mathrm{p}_{\mathrm{h}}^{\mathrm{K}} u_{\mathrm{h}}=0$ sur $\Omega$, alors $u_{\mathrm{h}}$ est nulle sur $R_{\mathrm{h}}^{\mathrm{K}}(\Omega)$. Si les suites $Q_{K}^{q} u_{h}$ sont nulles sur $R_{h}(\Omega)$ pour $q \leqslant K$, la suite $u_{h}$ est nuile sur $R_{h}^{K}(\Omega)$.

En effet, d'après le théorème 2-1 du \$2 :

$\mathrm{p}_{\mathrm{h}}^{\mathrm{K}} \mathrm{u}_{\mathrm{h}}=\sum_{\alpha \in R_{\mathrm{h}}(\Omega)} \sum_{\mathrm{q} \leqslant \mathrm{K}} u_{\mathrm{h}}^{\alpha+q} \psi_{\mathrm{K}}^{\mathrm{K}-\mathrm{q}}\left(\frac{\mathrm{x}}{\mathrm{h}}-\alpha\right) \mathrm{h}^{1 / 2} x_{h}^{\alpha}$

Si $p_{h}^{K} u_{h}=0$, pour tout $\alpha \in R_{h}(\Omega)$, nous avons :

$\sum_{q \leqslant K} u_{h}^{\alpha+q} \psi_{K}^{K-q}\left(\frac{x}{h}-\alpha\right)=0$ sur $\omega_{h}^{\alpha}: \alpha \varepsilon R_{h}(\Omega)$ 
Les polynômes $\psi_{K}^{K-q}\left(\frac{x}{h}-\alpha\right)$ étant linéairement indépendants, cela entraIne que :

$u_{h}^{\alpha+q}=0$ si $\alpha \in R_{h}(\Omega)$ et si $q \leqslant K$.

D'après (3-3), si $\beta \in R_{h}^{K}(\Omega)$, il existe $\alpha \in R(\Omega)$ et $q \leqslant K$ tel que $\beta=\alpha+q$. Donc $u_{h}^{B}=0$ quel que soit $B \in R_{h}^{K}(\Omega)$.

D'autre part, puisque :

$p_{h}^{K} u_{h}=\sum_{\alpha \in R(\Omega)} \sum_{q \leqslant K}\left(Q_{K}^{q} u_{h}\right)^{\alpha} \frac{\left(\frac{x}{h}-\alpha+1\right)^{q}}{q !} h^{1 / 2} x_{h}^{\alpha}$

si les $Q_{K}^{q} u_{h}$ sont nulles sur $R_{h}(\Omega), p_{h}^{K} u_{h}$ est nulle sur $\Omega$, donc $u_{h}$ est nulle sur $R_{h}^{K}(\Omega)$ d'après ce qui précède, ce qui démontre le théorème.

En particulier :

COROLLAIRE 3-1.

Sur l'espace des suites sur $R_{h}^{K}(\Omega)$, les expressions

$$
\left\|p_{h}^{K} u_{h}\right\|_{m, P, \Omega} \quad \text { sont des normes. }
$$

3-2. APPROXIMATIONS DES ESPACES $\mathrm{w}_{0}^{\mathrm{m}, \mathrm{P}}(\Omega)$.

Nous allons construire diverses approximations de l'espace de Sobolev $W_{0}^{m, P}(\Omega)$ en utilisant la construction des approximations de l'espace $W^{m, P}\left(R^{n}\right)$.

Soient $\mathrm{K}$ et $\mathrm{L}$ deux multientiers ; considérons le réseau :

(3-6) $\quad R_{h}=R_{h}^{K}(\Omega ; r)$

défini au $n^{\circ} 3-1$.

Nous désignerons par :

(3-7) $\quad \mathrm{W}_{\mathrm{O}, \mathrm{h}}^{\mathrm{m}, \mathrm{P}}(\Omega ; \mathrm{K})$

1'espace des suites $u_{h}=\left(u_{h}^{\alpha}\right)$ nulles en dehors de $R_{h}^{K}(\Omega ; r)$, muni de la norme $\left\|u_{h}\right\|_{h, m, P}$, définie aú $\$ 2 n^{0} 2(2,17)$. Si $K \geqslant(m)$, le prolongement $p_{h}^{K} u_{h}$ de la suite $u_{h}$, défini au $\$ 2 n^{\circ} 2(2,18)$, est à support compact dans $\Omega$.

Nous allons définir une restriction $\mathrm{r}_{\mathrm{h}}^{* \mathrm{~L}}$ de $\mathrm{w}_{\mathrm{o}}^{\mathrm{m}, \mathrm{P}}(\Omega)$ dans $\mathrm{w}_{\mathrm{O}, \mathrm{h}}^{\mathrm{m}, \mathrm{P}}(\Omega ; \mathrm{K})$ par : 
$(3-8) \quad\left(r_{h}^{* L} \phi\right)= \begin{cases}\left(\sqrt{h} \int \phi(x) x_{K+(1), h}^{\alpha}(x) d x\right) & \text { si } \alpha \in R_{h}^{K}(\Omega ; \Gamma) \\ 0 & \text { si } \alpha \notin R_{h}^{K}(\Omega ; \Gamma)\end{cases}$

Nous allons étudier les approximations :

(3-9) $\quad A_{h}^{K, L}\left(W_{0}^{m, P}(\Omega)\right)=\left(W_{0}^{m, P}(\Omega), W_{0, h}^{m, P}(\Omega ; K) ; p_{h}^{K}, r_{h}^{* L}\right)$ de $W_{0}^{m, P}(\Omega)$.

Posons :

$$
R_{\mathrm{h}}^{\mathrm{m}}=\bigcap_{|\mathrm{k}| \leqslant \mathrm{m}} \tau_{-\mathrm{k}} R_{\mathrm{h}} ; S_{\mathrm{h}}^{\mathrm{m}}=R_{\mathrm{h}}-R_{\mathrm{h}}^{\mathrm{m}} ; \Omega^{\mathrm{m}}(\mathrm{h})=\bigcup_{\alpha \varepsilon S_{\mathrm{h}}^{\mathrm{m}}} \omega_{\mathrm{L}, \mathrm{h}}^{\alpha}
$$

Supposons que l'ouvert $\Omega$ vérifie la propriété de régularité suivante :

$$
\left\{\begin{array}{l}
\text { Il existe } D=\left(D_{1}, \ldots, D_{n}\right) \text { tel que } \\
x-k h D \notin \Omega \text {. pour tout }|k| \leqslant m \text { et pour tout } x \in \Omega^{m}(h)
\end{array}\right.
$$

THEOREME 3-2.

Supposons l'hypothese $(3,11)$ satisfaite. Les approximations $A_{h}^{K, L}\left(w_{0}^{m, P}(\Omega)\right)$ sont alors stables et convergentes.

Si $\mathrm{K}=\mathrm{L}$, ces approximations sont de plus autoadjointes.

La stabilitê des prolongements résulte du théorème $2-1$, puisque :

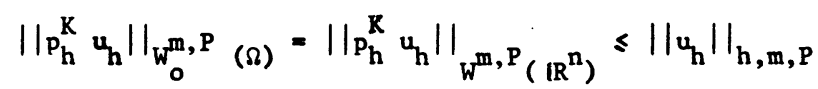

Nous allons montrer que les restrictions sont stables, c'est-à-dire qu'il existe une constante $\mathrm{C}$ indépendante de $\mathrm{h}$ telle que :

(3-12) $\left\|r_{h}^{* L} \phi\right\|_{h, m, P} \leqslant m|| p_{h}^{K} r_{h}^{* L} \phi\left\|_{m, P} \leqslant c\right\| \phi \|_{m, P}$.

Mais :

(3-13) $\mathrm{p}_{\mathrm{h}}^{\mathrm{K}} \mathrm{r}_{\mathrm{h}}^{* \mathrm{~L}} \phi=\sum_{\alpha \in R_{\mathrm{h}}}\left(\int_{\Omega} \phi x_{\mathrm{L}+(1), \mathrm{h}}^{\alpha} \mathrm{dx}\right) \mathrm{x}_{\mathrm{K}, \mathrm{h}} * \theta_{\mathrm{h}}^{\alpha}(\mathrm{x})$

où $\theta_{h}^{\alpha}$ est la fonction caractéristique du pavé $\omega_{h}^{\alpha}$.

La dérivée d'ordre $k=\left(k_{1}, \ldots, k_{n}\right),|k| \leqslant m$ de $p_{h}^{K} r_{h}^{* L} \quad$ s'écrit :

(3-14) $\quad D^{k} p_{h}^{K} r_{h}^{* L} \phi=X_{h}+Y_{h}$. 
97

oì

$$
x_{h}=\sum_{\alpha \in R_{h}^{m}}\left(\int_{\Omega} D^{k} \phi(x) \cdot x_{L+(1), h}^{\alpha} * \bar{x}_{k, h}(x) d x\right) x_{K-k, h} * \theta_{h}^{\alpha}(x)
$$

et

(3-16) $\quad Y_{h}=\sum_{\alpha \in S_{h}^{m}} h^{-k}\left\{\sum_{\ell \in k(x)}(-1)^{\ell} C_{k}^{\ell} \int_{\Omega} \phi(x) x_{L+(1), h}^{\alpha-\ell}(x) d x\right\} x_{K, h}^{*} \theta_{h}^{\alpha}(x)$

oì $k(\alpha)$ est l'ensemble des indices $\alpha-\ell$ qui appartiennent $a R_{h}$ pour $\mid \ell ! \leqslant k$

Nous savons que :

(3-17) $\int_{\Omega}\left|x_{h}\right|^{P} d x \leqslant \int_{\Omega}\left|D^{k} \phi(x)\right|^{P} d x$

Il nous reste à évaluer $\int_{\Omega}\left|\mathrm{Y}_{n}\right|^{\mathrm{P}} \mathrm{dx}$

Soit $\Omega^{m}(h)$ le sous-ensemble de $\Omega$ défini par :

$\Omega^{\mathrm{m}}(\mathrm{h})=\bigcup_{\alpha \varepsilon S_{\mathrm{h}}^{\mathrm{m}}} \omega_{\mathrm{L}, \mathrm{h}}^{\alpha}$

Il est alors clair que

$(3-18)\left\{\begin{array}{l}\int_{r}\left|Y_{h}\right|^{P} d x \leqslant M h^{-k P} \sum_{\alpha \varepsilon S_{h}^{m}}\left|\int \phi(x) x_{L, h} * \theta_{h}^{\alpha}(x) d x\right|^{P} \leqslant \\ \leqslant M h^{-k P} \sum_{\alpha \varepsilon S_{h}^{m}} \int|\phi(x)|^{P} x_{L, h} * \theta_{h}^{\alpha}(x) d x \leqslant \\ \leqslant M i^{-k P} \int_{\Omega^{m}(h)}|\phi(x)|^{P} d x .\end{array}\right.$

Nous allons majorer maintenant $|\phi(x)|^{P}$ lorsque $x \in \Omega^{m}(h)$.

D'après 1 'hypothèse (3-11), nous savons que :

(3-19) $x-k D h \& \Omega$ pour tout $|k| \leqslant m$ si $x \varepsilon \Omega^{m}(h)$

Soit :

(3-20) h $\psi_{h}$ la fonction caractéristique de $\prod_{1}\left(0, D_{1} h_{i}\right)$. Alors, si $\tilde{\phi}$ désigne le prolongement de $\$$ par 0 en dehors de $\Omega$,

$$
D^{k} \psi_{k, h} * \tilde{\phi}=h^{-k} \sum_{\ell \leqslant k}(-1)^{\ell} C_{k}^{\ell} \tilde{\phi}(x-\ell D h) ; \psi_{k, h}=\psi_{h} * \ldots * \psi_{h} \quad k \text {-fois. }
$$

7 
Donc, sous 1'hypothèse (3-11),

(3-21) $\phi(x)=h^{k} D^{k} \psi_{h}^{k} * \tilde{b}^{q} \quad$ sur $\Omega^{\mathrm{m}}(\mathrm{h})$.

Il en résulte alors quue :

(3-22) $\int_{\Omega^{\mathrm{m}}(\mathrm{h})}|\phi(\mathrm{x})|^{\mathrm{P}} \mathrm{dx}=h^{\mathrm{kP}}\left\|\psi_{\mathrm{h}}^{\mathrm{k}} \times \mathrm{D}^{\mathrm{k}} \tilde{\phi}\right\|_{\mathrm{P}}^{\mathrm{P}} \leqslant \mathrm{N} \mathrm{h}^{\mathrm{kP}} \| \mathrm{D}^{\mathrm{k}} \tilde{\phi}_{\|_{P}}^{\mathrm{P}}$

Puisque $\left\|\mathrm{D}^{\mathrm{k}} \tilde{\phi}\right\|_{\mathrm{p}}=\left\|\mathrm{D}^{\mathrm{k}} \phi\right\|_{L^{\mathrm{p}}(\Omega)}$, on déduit de (3-22) et de (3-18) que :

(3-23) $\int\left|\mathrm{Y}_{\mathrm{h}}^{\mathrm{P}}\right| \mathrm{dx} \leqslant \mathrm{C}|| \mathrm{D}^{\mathrm{k}} \phi \|_{\mathrm{P}}^{\mathrm{P}}$

ce qui achève la démonstration de la stabilité des restrictions $r_{h}^{* L}$.

La convergence de ${ }_{p_{h}^{K}}^{K} x_{h}^{* L} \phi$ vers $\phi$ est alors immédiate. Puisque les troncatures $p_{h}^{K} r_{h}^{* L}$ sont uniformément bornées, il suffit de vérifier la convergence lorsque $\phi$ est à support compact $K$ dans $\Omega$. Dans ce cas, pour $h$ assez petit,

$\mathrm{K} \cap \Omega^{\mathrm{m}}(\mathrm{h})=\emptyset$ et alors :

$p_{h}^{K} r_{h}^{* L} \phi=p_{h}^{K} r_{h}^{* L} \tilde{\phi}$ converge vers $\tilde{\phi}$ dans $w^{m, p}\left(\mathbb{R}^{n}\right)$

Lorsque $\mathrm{K}=\mathrm{L}$, il est évident que les approximations sont auto-adjointes. Dans ce cas, les approximations :

$$
A_{h}^{K}\left(w^{-m, p^{\prime}}(\Omega)\right)=\left(w^{-m, p^{\prime}}(\Omega), W_{h}^{-m, p^{\prime}}(\Omega, K), p_{h}^{K}, r_{h}^{* K}\right) \text { sont également }
$$

stables et convergentes.

Nous allons maintenant définir des approximations partielles de $w_{0}^{\mathrm{m}, \mathrm{p}}(\Omega)$. (cf. chapitre I, $\$ 1, n^{0} 1-4$ ).

Soit $\vec{K}=\left(\mathrm{K}_{q}\right)_{|q| \leqslant m}$ une famille de multientiers $\mathrm{K}^{q}$, et $\mathrm{K}=\sup _{|q| \leqslant m} K_{q} \cdot$

Considérons les espaces $W_{0}^{q, p}$. Si $K_{q} \geqslant q$, les prolongements : $p_{h}^{K_{q}}$ sont défi-

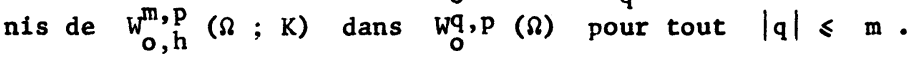

THEOREME 3-3.

Supposons que l'ouvert $\Omega$ vërifie l'hypothèse (3-11). Alors les approximations des espaces $\mathrm{w}_{\mathrm{O}}^{\mathrm{q}, \mathrm{p}}(\Omega)$ dans $\mathrm{w}_{\mathrm{O}, \mathrm{h}}^{\mathrm{m}} \mathrm{p}(\Omega ; \mathrm{K})$

(3-24) $\quad A_{h}^{\vec{K}, L}\left(w_{o}^{q, p}(\Omega)\right)=\left(w_{0}^{q, p}(\Omega) ; w_{0, h}^{m, p}(\Omega ; K), p_{h}^{K_{q}}, r_{h}^{* L}\right)$

sont des approximations partielles de $w_{0}^{m, p}(\Omega)$ et les approxinations $A_{h}^{K, L}\left(W_{0}^{m, p}(\Omega)\right)$ 
sont plus fines que ces approximations partielles.

En effet, si pour tout $|q| \leqslant m$, les $p_{h}^{K_{q}} u_{h}$ convergent faiblement vers $u_{q}$ dans $L^{p}(\Omega)$, alors $p_{h}^{K} u_{h}=x_{K-K}, h * p_{h}^{K_{q}} u_{h}$ converge faiblement vers $u_{q}$ pour tout $q$, ce qui entraf̂ne que tous les $u_{\text {g }}$ sont égaux à un même élément u . D'autre part, $D^{q} u=D^{q} u_{q}$ appartient à $L^{p}(\Omega)$ et $u$ est à support compact. Donc u appartient à $W_{0}^{m, p}(\Omega)$ et les approximations $A_{h}^{K, L}\left(W_{0}^{m}, p(\Omega)\right)$ sont des approximations partielles de $w_{0}^{m, p}(\Omega)$, qui, d'après le théorème 3-2, sont convergentes.

D'après le théorème $2-1$ du $\$ 2$, nous savons que : (3-25) $\quad|| p_{h}^{K} u_{h}\left\|_{m, p} \leqslant\right\| u_{h} \|_{h, m, p} \leqslant m\left(\sum_{|q| \leqslant m}\left|D^{q} p_{h}^{K} u_{h}\right|_{p}^{p}\right)^{\frac{1}{p}}$

Pour montrer que les approximations $A_{h}^{K, L}\left(w_{0}^{m, p}(\Omega)\right)$ sont plus fines que les approximations partielles, il ne reste plus qu'à vérifier que : $\lim _{h=0}\left(p_{h}^{K} u_{h}-p_{h}^{K} u_{h}\right)=0$ dans $w_{0}^{q, p}(\Omega)$ faible si $\left\|u_{h}\right\|_{h, m, p} \leqslant$ Cste. Mais, puisque $W_{0}^{q, p}(\Omega)$ est dense dans $L^{P}(\Omega)$, alors $L^{P^{\prime}}(\Omega)$ est dense dans le dual de $\mathrm{W}_{0}^{\mathrm{q}, \mathrm{p}}(\Omega)$ et 11 suffit de vérifier que, pour toute $\phi$ de $\mathrm{L}^{\mathrm{P}^{\prime}}(\Omega)$,

(3-26) $\left.\lim _{h=0} \mid p_{h}^{K} u_{h}-p_{h}^{K_{q}} u_{h}, \phi\right) \mid=0$

Mais cela résulte de 1 'inégalité :

$$
\begin{aligned}
& \left|\left(p_{h}^{K} u_{h}-p_{h}^{K_{q}} u_{h}, \phi\right)\right|=\left|\left(p_{h}^{K_{q}} u_{h}, \tilde{\phi}-x_{K-K}, h * \tilde{\phi}\right)\right| \leqslant \\
& \leqslant\left.\left\|p_{h}^{K_{q}} u_{h}\right\|\right|_{q, p}|| \tilde{\phi}-x_{K-K_{q}, h} * \tilde{\phi} \|_{P^{\prime}}
\end{aligned}
$$

3-3. APPROXIMATIONS DES ESPACES $W^{\mathrm{m}, \mathrm{p}}(\Omega)$.

L'espace $D(\Omega) \quad n^{\prime}$ est pas dense dans $W^{\mathrm{m}, \mathrm{p}}(\Omega)$. Néanmoins, si l'ouvert $\Omega$ est suffisamment régulier, nous allons construire des approximations $W^{\mathrm{m}, \mathrm{P}}(\Omega)$ à partir

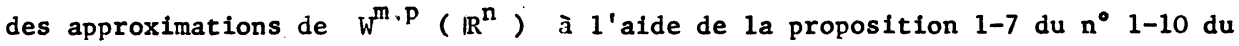
$\$ 1$ du chapitre I.

On dira qu'un ouvert $\Omega$ a la propriété de m-prolongement s'il existe un opérateur continu $\pi$ de $W^{\mathrm{m}, \mathrm{p}}(\Omega)$ dans $W^{\mathrm{m}, \mathrm{p}}\left(\mathbb{R}^{\mathrm{n}}\right)$ tel que, si $\rho$ désigne l'opérateur de restriction à $\Omega$, on ait :

$$
\rho \pi=1
$$


Pour des exemples d'ouverts $\Omega$ ayant la propriété de m-prolongement, on pourra consulter J.L. LIONS [1] , chapitre II, où on trouvera la bibliographie nécessaire.

Considérons des approximations de $W^{m, p}$ définies au $n^{\circ} 2-2$ du $\$ 2$ :

$A_{h}^{K}\left(w^{m, p}\right)=\left(w^{m, p}, w_{h}^{m, p}, p_{h}^{K}, r_{h}\right) ; K \geqslant(m)$

Désignons maintenant par $w_{h}^{m, p}(\Omega ; K) \quad l^{\prime}$ 'espace des suites $u_{h}=\left(u_{h}^{\alpha}\right)$ définies $\operatorname{sur} R_{h}^{K}(\Omega)\left(\right.$ cf. $\left.n^{0} 3-1\right)$ muni de la norme $\left\|p_{h}^{K} u_{h}\right\|_{m, p, \Omega}$

Posons :

$(3-27)\left\{\begin{array}{l}r_{h, \Omega}(\phi)=r_{h}(\pi \phi) \\ p_{h, \Omega}^{K} u_{h}=\rho\left(p_{h}^{K} u_{h}\right)\end{array}\right.$

Il est clair qu'une suite, $u_{h}$ définie sur $R_{h}^{K}(\Omega)$ peut toujours être considérée comme la restriction à $R_{h}^{K}(\Omega)$ d'une suite définie sur $z^{n}$ (11 suffit de $1 a$ prolonger par 0 par exemple) et que $p_{h, \Omega}^{K} u_{h}$ ne dépend pas du choix de ce prolongement.

On déduit du théorème 2-1 du $\$ 2$ et de la proposition 1-7 du $\$ 1$ du chapitre I le

THEOREME 3-4.

Les approximations :

$A_{h}^{K}\left(w^{m, p}(\Omega)\right)=\left(w^{m, p}(\Omega), w_{h}^{m, p}(\Omega ; k), p_{h, \Omega}^{K}, r_{h, \Omega}\right)$

sont stables et convergentes. De plus :

(3-28) $\left\{\begin{array}{c}D^{q} p_{h, \Omega}^{K} u_{h}=p_{h, \Omega}^{K-q} \nabla_{h}^{q} \quad \text { sur } \Omega \\ \left\|D^{q}\left(p_{h, \Omega}^{K} r_{h, \Omega} \phi-\phi\right)\right\|_{p} \leqslant C \omega_{p}\left(D^{q} \pi \phi, h\right)\end{array}\right.$

AUTRES NORMES SUR $w_{h}^{m, p}(\Omega ; k)$

Soit L un multientier. Nous poserons :

(3-29) $\left|u_{h}\right|_{h, p,(L)}=h^{1 / p}-1 / 2\left(\sum_{\alpha \in R_{h}^{L}(\Omega)}\left|u_{h}^{\alpha}\right|^{p}\right)^{1 / p}$ 
Nous obtenons alors les inégalités suivantes :

PROPOSITION 3-1.

$(3-30)\left\{\begin{array}{c}\left.|| p_{h}^{K} u_{h}\right|_{m, p, \Omega} \leqslant\left(\sum_{|q| \leqslant m}\left|\nabla_{h}^{q} u_{h}\right|_{h, p,(K-q)}^{p}\right)^{1 / p} \leqslant \\ \leqslant c\left(\sum h^{-P q}\right)^{1 / p}\left|u_{h}\right|_{h, p,(K) .}\end{array}\right.$

En effet, on remarque que :

$$
\begin{aligned}
& \left\|p_{h}^{K} u_{h}\right\|_{P, \Omega}^{P}=h^{-P / 2} \int_{r}\left|\sum u_{h}^{\alpha} x_{K, h}^{\alpha} * \theta_{h}^{\alpha}\right|^{P} d x \leqslant \\
& \leqslant n^{-P / 2} \sum_{\alpha \in R_{h}^{K}(\Omega)}\left|u_{h}^{\alpha}\right|^{P} \int_{\Omega} x_{K, h}^{\alpha} * \theta_{h}^{\alpha} d x=h^{1-P / 2} \sum_{\alpha \in R_{h}^{K}(\Omega)}\left|u_{h}^{\alpha}\right|^{P}
\end{aligned}
$$

La proposition en découle en remarquant que $D^{q} p_{h}^{K}=p_{h}^{K-q} \nabla_{h}^{q}=h^{-q} p_{h}^{K-q} \nabla^{q}$. APPROXIMATIONS PARTIELLES DE $W^{\mathrm{m}, \mathrm{P}}(\Omega)$.

Rappelons que $W^{m, P}(\Omega)$ est $1^{\prime}$ intersection des espaces $W^{q, P}(\Omega)$ pour $|q| \leqslant m$, où $W^{q, P}(\Omega)$ est $l^{\prime}$ espace des fonctions $u$ de $L^{P}(\Omega)$ dont la dérivée $D^{q} u$ appartient à ${ }_{L}^{P}(\Omega)$.

Solt $\vec{K}=\left(K_{q}\right)|q| \leqslant m$ une famille de multientiers $K_{q}$, vérifiant :

(3-31) $\quad \mathrm{K}_{\mathrm{q}}=\mathrm{K}_{\mathrm{o}}+\mathrm{q}$

Les cas les plus intéressants étant ceux où $K_{q}=q$. Nous allons associer à $\vec{k}$ des approximations partielles de $W^{m, P}(\Omega)$ (cf. chapitre I; $\$ 1, n^{\circ} 1-4$ ).

Considérons les réseaux : (cf. $\mathrm{n}^{\circ} 3-1$ )

(3-32) $\quad R_{\mathrm{h}}^{\overrightarrow{\mathrm{k}}}(\Omega)=\bigcup_{|\mathrm{q}| \leqslant \mathrm{m}} R_{\mathrm{h}}^{\mathrm{K}} \mathrm{q}(\Omega) ; R_{\mathrm{m}}^{\overrightarrow{\mathrm{m}}}(\Omega)=\bigcup_{|\mathrm{q}| \leqslant \mathrm{m}} R_{\mathrm{h}}^{\mathrm{q}}(\Omega)$

On désigñe maintenant par $w_{h}^{m}, P(\Omega ; \vec{K}) \quad l$ 'espace des suites $u_{h}=\left(u_{h}^{\alpha}\right)$ définies sur $R_{h}^{\vec{R}}(\Omega)$, et on pose :

(3-33) $\quad p_{h, \Omega}^{K_{q}} \quad u_{h}=\rho\left(p_{h}^{K_{q}} u_{h}\right) ; r_{h, \Omega}(\phi)=r_{h}(\pi \phi)$ 
THEOREME 3-5.

Sous l'hypathèse $(3-31)$, les approximations:

$\left.A_{h}^{\vec{K}}\left(w^{q, P}(\Omega)\right)=\left(w^{q, P}(\Omega)\right) w_{h}^{m, P}(\Omega ; \vec{K}), p_{h, \Omega}^{K}, r_{h, \Omega}\right)$

sont des approximations partielles de $\mathrm{w}_{\mathrm{h}}^{\mathrm{m}, \mathrm{P}}(\Omega: \overrightarrow{\mathrm{K}})$, stables et convergentes, et : (3-34) $\quad|| u_{h} \|_{h, p, \vec{K}}=\left(\sum_{|q| \leqslant m}\left|D^{q} p_{h, \Omega}^{K} u_{h}\right|_{p}^{p}\right)^{1 / p}$

est une norme sur $\mathrm{w}_{\mathrm{h}}^{\mathrm{m}, \mathrm{P}}(\Omega ; \overrightarrow{\mathrm{k}})$.

Nous allons tout d'abord montrer que $\left\|u_{h}\right\|_{h, p, \vec{K}}$ est une norme $\operatorname{sur} w_{h}^{m, P}(\Omega ; \vec{k})$. Supposons que $\left\|u_{h}\right\|_{h, p, \vec{k}}=0$. Alors :

$$
p_{h}^{K_{0}} u_{h}=0 \text { sur } \Omega ; \quad p_{h}^{K_{q}-q}\left(\nabla_{h}^{q} u_{h}\right)=p_{h}^{K_{0}}\left(\nabla_{h}^{q} u_{h}\right)=0 \text { sur } \Omega .
$$

Donc, d'après le théorème 3-1, les $\nabla_{h}^{q} u_{h}$ sont nuls sur $R_{h}^{K_{0}}(\Omega)$ pour tout $|q| \leqslant m$, ce quil entraine que $u_{h}$ est nulle sur $R_{h}^{\vec{k}}(\Omega)$. En effet :

(3-36) $\quad R_{h}^{\vec{K}}(\Omega)=\bigcup_{|q| \leqslant m} \tau_{q} R_{h}^{K_{0}}(\Omega)=\bigcup_{|\mathrm{k}| \leqslant 1} \tau_{k} \bigcup_{|q| \leqslant m-1} \tau_{q} R_{h}^{K_{0}}(\Omega)$

et 11 suffit, par récurrence, de montrer le résultat pour $m=1$. Supposons que $\alpha$ appartient à $\bigcup_{|k| \leqslant 1} \tau_{k} R_{h}^{K_{0}}(\Omega)$. Si a n'appartient pas à $R_{h}^{K_{0}}(\Omega)$, il existe $e_{i}$ tel que $\alpha-e_{i}$ appartient à $R_{h}^{K_{0}}(\Omega)$. Alors

$$
\left(\nabla_{h_{i}} u_{h}\right)^{\alpha-e_{i}}=h_{i}^{-1}\left(u_{h}^{\alpha}-u_{h}^{\alpha-e_{i}}\right)=0 \text {. }
$$

Donc $u_{h}^{\alpha}=u_{h}^{\alpha-e_{1}}=0$, ce qui établit la dernière partie du théorème.

Supposons maintenant que $p_{h}^{K_{q}} u_{h}$ converge faiblement vers $u_{q}$ dans $W^{q, P}(\Omega)$ pour tout $q$. Montrons alors que

(3-38) $\quad D^{q} u_{0}=D^{q} u_{q}$ pour tout $q$.

En effet :-

(3-39) $\quad \nabla_{h}^{q} p_{h}^{K_{O}} u_{h}=D^{q} \chi_{h}^{q} * p_{h}^{K_{0}} u_{h}=D^{q} p_{h}^{K_{q}} u_{h}$

Donc, puisque $\mathrm{p}_{\mathrm{h}, \Omega}^{\mathrm{K}_{\mathrm{O}}} \mathrm{u}_{\mathrm{h}}$ et $\mathrm{p}_{\mathrm{h}}^{\mathrm{K}_{\mathrm{q}}} u_{\mathrm{h}}$ convergent dans $D^{\prime}(\Omega)$, pour touté $\phi$ à support compact et pour $h$ assez petit, on aura :

$$
\left(\nabla_{h}^{q} p_{h, \Omega}^{k_{o}} u_{h}, \phi\right)=(-1)^{|q|}\left(p_{h}^{K_{0}} u_{h}, \nabla_{h}^{q} \phi\right)=(-1)^{|q|}\left(p_{h}^{K_{q}} u_{h}, D^{q} \phi\right)
$$


Donc, lorsque $h$ tend vers 0 , nous aurons :

(3-40) $\quad D^{q} u_{q}=D^{q} u_{0} \& L^{P}(\Omega)$.

On en déduit donc que $u_{0}=u_{q}$ (pour tout $|q| \leqslant m$ ) appartient à $w^{m, P}(\Omega)$, ce qui montre la première partie du théorème.

La convergence des approximations partielles résulte alors du théorème 3-4 .

EXEMPLE 3-1 APPROXIMATIONS DE $W^{\mathrm{m}, \mathrm{P}}(0, \mathrm{~T})$.

Considérons le cas où $n=1$ et prenons pour ouvert $\Omega 1$ 'ouvert $(0, T)$ et $h=T / N$, où $N$ est un entier destiné à tendre vers 1 'infini. Soit $K$ un entier positif. Alors :

$(3-41)\left\{\begin{array}{l}R_{\mathrm{h}}^{\mathrm{K}}(\Omega)=\{1,2, \ldots, \mathrm{N}+\mathrm{K}\} \\ R_{\mathrm{h}}^{\mathrm{K}}(\mathrm{r})=\{1,2, \ldots, \mathrm{K} ; \mathrm{N}+1, \ldots, N+\mathrm{K}\}\end{array}\right.$

L'espace $W^{\mathrm{m}, \mathrm{P}}(\Omega ; \mathrm{K})$ est un espace de dimension $\mathrm{N}+\mathrm{K}$ et

(3-42) $\quad p_{h, \Omega}^{K} u_{h}=h^{1 / 2} \sum_{\alpha=1}^{N+K} x_{h}^{\alpha}\left(\sum_{q=0}^{K}\left(o_{K}^{q} u_{h}\right)^{\alpha}\left(\frac{x}{h}-\alpha+1\right)^{q}\right)$

Si $K=1$, il est clair que :

(3-43) $\quad p_{h}^{1} u_{h}=p_{h}^{0} u_{h}+h \sum_{\alpha}\left(\nabla_{h} u_{h}\right)^{\alpha}\left(\frac{x}{h}-\alpha+1\right) h^{1 / 2} x_{h}^{\alpha}$

On en déduit par recurrence une relation entre les $p_{h}^{K}$ :

(3-44) $\quad p_{h}^{K} u_{h}=\sum_{k=1}^{m} \frac{h^{k-1}}{k !} p_{h}^{K-k}\left(\nabla_{h}^{k-1} u_{h}\right)+\frac{h^{K}}{k !} \sum_{\alpha}\left(\nabla_{h}^{K} u_{h}\right)^{\alpha}\left(\frac{x}{h}-\alpha+1\right)^{K} h^{1 / 2} x_{h}^{\alpha}$

Pour définir la restriction $r_{h, \Omega}=r_{h} \pi$, nous allons prendre pour prolongement $\pi$ le prolongement de Babitch - Hestenes :

$(3-45) \pi \phi(x)=\left\{\begin{array}{l}\sum_{j=1}^{m} \lambda_{j} \phi(-j x \omega) \text { si }-\frac{T}{m \omega} \leqslant x \leqslant 0 \\ \phi(x) \text { si } x \in[0, T] \\ \cdot \sum_{j=1}^{m} \lambda_{j} \phi(j \omega(x-T)) \text { si } T \leqslant x \leqslant T\left(1+\frac{1}{m \omega}\right)\end{array}\right.$

où les $\lambda_{j}$ sont solutions du système : 
(3-46) $\sum_{j=1}^{m}(-j \omega)^{k} \lambda_{j}=1 \quad$ pour $k=0, \ldots, m-1$.

Si $\phi$ est la restriction à $(O, T)$ d'une fonction $\phi$ définie sur la droite, on pourra prendre alors $\pi \phi=\phi$. On remarque que dans ce cas 1a troncature

$p_{h, \Omega}^{K} r_{h} \phi$ d'une fonction $\phi$ est positive si $\phi$ est positive, monotone si $\phi$ est monotone, convexe si $\phi$ est convexe.

3-4. APPROXIMATIONS DE SOUS-ENSEMBLES CONVEXES DES ESPACES DE SOBOLEV.

EXEMPLE 3-1.

Soit $E$ un sous-ensemble fermé d'un ouvert $\Omega$. Considérons $l$ 'ensemble convexe ferm $\bar{e} \mathrm{~K}$ des fonctions de $\mathrm{w}_{0}^{\mathrm{m}, \mathrm{P}}(\Omega)$ plus grandes que 1 (presque partout) sur $\mathrm{E}$.

Nous allons construire des approximations et des approximations partielles de cet ensemble $K$.

Soit $L$ un multientier plus grand que $(m)$. Considérons 1 'espace $w_{0, h}^{m, P}(\Omega ; L)$ défini au $n^{\circ} 3-2$ et le prolongement $p_{h}^{L}$.

Nous prendrons pour sous-ensemble convexe fermé $K_{h}$ de $w_{0, h}^{m, P}(\Omega ; L)$ le sousensemble $k_{h}$ des suites $u_{h}$ telles que (3-47) $u_{h}^{\alpha} \geqslant h^{+1 / 2}$ pour tout $\alpha$ de $R_{h}^{L}$ (E).

Nous supposerons $E$ assez rëgulier pour que toute fonction $\phi$ plus grande que 1 sur $E$ soit limite dans $w_{0}^{m, P}(\Omega)$ de fonctions $\gamma_{h} \phi$ plus grandes que 1 sur $\mathrm{E}_{\mathrm{n}}$, où

(3-48) $\quad E_{h}=\left\{\bigcup \omega_{h}^{\alpha} ; \alpha \varepsilon R_{h}^{L}(E)\right\}$

Nous poserons alors :

(3-49) $\quad r_{h} \phi=r_{h}^{* 1}\left(r_{h} \phi\right)$

THEOREME 3-6.

Les prolongements $p_{h}^{L}$ et les restrictions $r_{h}$ vérifient:

(3-50) $\quad \mathrm{p}_{\mathrm{h}}^{\mathrm{L}} \mathrm{K}_{\mathrm{h}} \subset \mathrm{K} \quad ; \quad \mathrm{r}_{\mathrm{h}} \mathrm{K} \subset \mathrm{K}_{\mathrm{h}}$

(3-51) $\lim _{h=0}\left\|p_{h}^{L} r_{h} \phi-\phi\right\|_{m, P}=0$ pour toute $\phi \varepsilon \mathrm{K}$. 


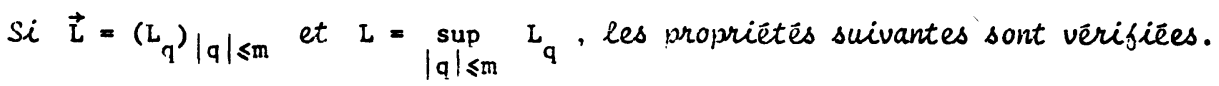
(3-52) $\left\{\begin{array}{c}\text { si } u_{h} \in k_{h} \text { et } s i p_{h}^{L_{q}} u_{h} \text { converge jaiblenent vers } u_{q} \text { dans } w_{o}^{q, p}(\Omega) \\ \text { pour tout }|q| \leqslant m \text {, alors } u_{q}=u \text { appartient à } k\end{array}\right.$

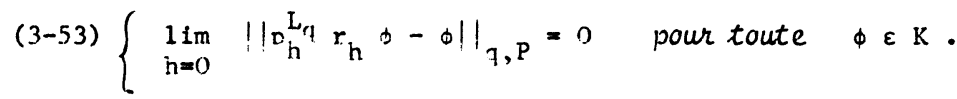

Le seul point à vérifier est (3-50), le reste du théorème se déduisant des résultats nrécédents.

Puis gue $h^{1 / 2} \sum x_{L+(1), h}^{\alpha} \geqslant n^{-1 / 2}$, on en déduit que :

$n_{h}^{L} u_{h} !_{E}=\left.\sum_{\alpha \in R_{h}^{L}(\Omega)} u_{h}^{\alpha} h^{1 / 2} x_{L+(1), h}^{\alpha}\right|_{E} \geqslant h^{-1 / 2}$ sup $u_{h}^{\alpha} \geqslant 1$

d'où 11 résulte que $p_{h}^{L} K_{h} \subset k$.

Montrons que $r_{h} K \subset K_{h}$. Soit $\phi$ une fonction plus grande que, 1 sur $F$. Alors $\gamma_{h} \phi$ est plus grande que 1 sur $E_{h}$ et si $\alpha \in R_{h}^{L}(\Omega)$, $\left(x_{h} \phi\right)^{\alpha}=h^{+1 / 2} \int\left(r_{h} \phi\right) x_{h}^{\alpha} d x \geqslant h^{1 / 2} \int x_{h}^{\alpha} d x \geqslant h^{+1 / 2}$

EXEMPLE 3-2.

Considérons maintenant le cône convexe fermê $\mathrm{K}$ de $\mathrm{w}^{\mathrm{m}}, \mathrm{P}(\Omega)$ des fonctions $\phi$ de $\mathrm{w}^{\mathrm{m}}, \mathrm{P}(\Omega)$ telles que la restriction de $\phi$ à la frontière $\Gamma$ de $\Omega$ soit positive (presque partout).

Nous allons construire des approximations et des approximations partielles de ce cône convexe.

Soit $L$ un multientier plus grand que $(m)$. Considérons l'espace $W^{m, P}(\Omega, L)$ défini au $n^{0} 3-3$ et le prolongement $p_{h, \Omega}^{L}=\rho p_{h}^{L}$.

Nous prendrons pour cône convexe fermé $K_{h}$ de $W_{h}^{m, P}(\Omega, L)$ le sous-ensemble $\mathrm{K}_{\mathrm{h}}$ des suites $\mathrm{u}_{\mathrm{h}}$ telles que

(3-54) $u_{h}^{\alpha} \geqslant 0$ pour tout $\alpha \varepsilon R_{h}^{L}(r)$. 
Nous supposerons que l'ouvert $\Omega$ est suffisanment régulier pour que toute fonction $\phi$ dont la trace sur $\Gamma$ soit positive soit linite d'une fonction $\gamma_{h} \phi$ de $W^{\mathrm{m}}, \mathrm{P}\left(\mathrm{R}^{\mathrm{n}}\right)$ positive sur :

(3-55) $\quad \Gamma_{h}=\left\{\bigcup \omega_{h}^{\alpha} ; \alpha \in R_{h}^{L}(r)\right\}$

Nous poserons alors

(3-56) $\quad r_{h} \phi=r_{h}^{* 1}\left(r_{h} \phi\right)$

Si $\overrightarrow{\mathrm{L}}=\left(\mathrm{L}_{\mathrm{q}}\right)|\mathrm{q}| \leqslant \mathrm{m}$ avec :

(3-57) $\quad L_{q}=L_{0}+q$ pour $|q| \leqslant m$,

Nous prendrons pour espace $V_{h}$ l'espace $W_{h}^{m, P}(\Omega, \vec{L})$ et pour cône $K_{h}$ l'ensemble des suites $u_{h}$ positives sur $R_{h}^{L_{0}}(\Gamma)$, pour restriction $r_{h}$ la restriction $r_{h}^{* 1}\left(\gamma_{h} \phi\right)$.

THEOREME 3-7.

Les prolongenents $p_{h, \Omega}^{L}$ et les restrictions $r_{h}$ vérifient

(3-58) $\left\{\begin{array}{l}\mathrm{p}_{\mathrm{h}, \Omega}^{\mathrm{L}} \mathrm{K}_{\mathrm{h}} \subset \mathrm{K}, \mathrm{r}_{\mathrm{h}} \mathrm{K} \subset \mathrm{K}_{\mathrm{h}} \\ \lim _{\mathrm{h}=0}\left\|\mathrm{p}_{\mathrm{h}, \Omega}^{\mathrm{L}} \mathrm{r}_{\mathrm{h}} \phi-\phi\right\|_{\mathrm{m}, \mathrm{P}, \Omega}=0 \text { pour toute } \phi \text { dans } \mathrm{K} .\end{array}\right.$

Si $\vec{L}=\left(L_{q}\right)|q| \leqslant m$ avec $L_{q}=L_{0}+q$, alors

(3-59)

1) $\left\|u_{h}\right\|_{h}=\left(\sum_{|q| \leqslant m}\left|D^{q} p_{h, \Omega}^{L_{q}} u_{h}\right|_{p}^{p}\right)^{1 / p}$ est une norme sur $v_{h}$.

ii) Si $u_{h} \in K_{h}$ et si $p_{h, \Omega}^{L_{q}} u_{h}$ converge faiblement vers $u_{q}$ dans $\mathrm{w}^{\mathrm{q}, \mathrm{P}}(\Omega)$ pour tout $|\mathrm{q}| \leqslant \mathrm{m}$, alors $\mathrm{u}_{\mathrm{q}}=\mathrm{u}$ appartient $\bar{a} \cdot \mathrm{K}$.

iii) $\lim _{\mathrm{h}=0}\left\|\mathrm{p}_{\mathrm{h}}^{\mathrm{L}_{\mathrm{q}}} \mathrm{r}_{\mathrm{h}} \phi-\phi\right\|_{\mathrm{q}, \mathrm{P}, \Omega}=0$ pour toute $\phi$ dans $\mathrm{K}$.

iv) $r_{h} K \subset k_{h}$ :

Si $u_{h}^{\alpha} \geqslant 0$ pour tout $\alpha \in R_{h}^{L}(r)$, il est clair que

$\left.p_{h, \Omega}^{L} u_{h}\right|_{\Gamma}=\left.\sum_{\alpha} u_{h}^{\alpha} h^{1 / 2} x_{L+(1), h}^{\alpha}\right|_{\Gamma} \geqslant 0$ 
donc $\mathrm{p}_{\mathrm{h}, \Omega}^{\mathrm{L}} \mathrm{K}_{\mathrm{h}} \subset \mathrm{K}$.

De même, si $\phi \varepsilon K$, alors $\gamma_{h} \phi$ est positive sur $\Gamma_{h}$ et, si $\alpha \varepsilon R_{h}^{L}(\Gamma)$

$\left(r_{h} \phi\right)^{\alpha}=h^{1 / 2} \int \gamma_{h} \phi x_{h}^{\alpha} d x \geqslant h^{1 / 2} \int x_{h}^{\alpha} d x \geqslant 0$.

ce qui entraine que $r_{h} K \subset K_{h}$.

I1 nous reste à montrer (3-59) ii) et plus précisément que u $\varepsilon \mathrm{K}$. Mais on sait (théorème 3-5) que $u \in W^{m, P}(\Omega)$ et que $u=\lim _{h=0} p_{h}^{L_{O}} u_{h}$ dans $L^{P}(\Omega)$. Mais, puisque $p_{h}^{L_{0}} u_{h}$ est positive sur $\Gamma$, u est positive sur $\Gamma$, donc appartient à $\mathrm{K}$.

\$4. APPROXIMATION DES'SOLUTIONS DES PROBLEMES AUX LIMITES POUR DES OPERATEURS DIFFERENTIELS LINEAIRES OU NON •

Ce paragraphe est consacré aux applications des résultats abstraits des $\$ 3$ et $\$ 4$ du chapitre I et à ceux du $\$ 3$ de ce chapitre.

$\mathrm{Au} \mathrm{n}^{\circ}$ 4-1, nous rappelons quelques résultats sur la formulation variationnelle des problèmes aux limites pour les opérateurs différentiels elliptiques d'ordre $2 \mathrm{~m}$. Au $\mathrm{n}^{\bullet} 4-2$, en utilisant les approximations du $\mathrm{n}^{\bullet} 3-3 \mathrm{du} \$ 3 \cdot$, nous énonçons les théorèmes de convergence des solutions approchées de ces problèmes aux limites. Si $m>1$, nous obtenons des résultats nouveaux, car nous déduisons la coercivité des problèmes approchés de la coercivité du problème initial, sans avoir besoin de 1 'établir directement. Nous explicitons les coefficients des matrices des opérateurs approchés. Nous retrouvons en particulier les schémas classiques. Au n ${ }^{\circ}$ 4-3, nous considérons des problèmes non linéalres, et, au $n^{\circ} 4-4$, des applications du $\$ 4$ du chapitre I. Nous approchons ainsi les solutions de problèmes aux limites unilatéraux et le calcul des capacités. Pour ne pas alourdir l'exposé, nous avons limité le nombre des applications.

4-1. RAPPELS SUR LES PROBLEMES ELLIPTIQUES D'ORDRE $2 \mathrm{~m}$.

Nous rappelons dans ce numéro les résultats concernant les problèmes aux limites elliptiques d'ordre $2 \mathrm{~m}$. (cf. J.L.LIONS [1], [2] ).

Soit $\Omega$ un ouvert de $R^{n}$. Prenons :

$(4-1)\left\{\begin{array}{l}\mathrm{H}=\mathrm{L}^{2}(\Omega) \\ \mathrm{V} \text { un sous-espace vectoriel fermé de } \mathrm{H}^{\mathrm{m}}(\Omega) \text { tel que } \mathrm{H}_{\mathrm{o}}^{\mathrm{m}}(\Omega) \subset \mathrm{V} \subset \mathrm{H}^{\mathrm{m}}\end{array}\right.$ 
$\left(H^{m}(\Omega)=W^{m, 2}(\Omega)\right)$

Considérons la forme sesquilinéaire suivante :

(4-2) $\quad a(u, v)=|p|,|q| \leqslant m) \int_{\Omega} a_{p q}(x) D^{p} u \overline{D^{q} v} d x ; a_{p q}(x) \in L^{\infty}(\Omega)$.

et considérons le triplet $A=\{\mathrm{V}, \mathrm{B}, \mathrm{a}(\mathrm{u}, \mathrm{v})\}$ (cf. Appendice)

Il est clair que $V$ étant un sous-espace fermé de $\mathrm{H}^{\mathrm{m}}(\Omega)$, la forme $\mathrm{a}(\mathrm{u}, \mathrm{v})$ est continue sur $D(\Omega)$ et définit l'opérateur A suivant à valeurs dans $D^{\prime}(\Omega)$ : (4-3) A u $=\sum_{|p|,|q| \leqslant m}(-1)|q|_{D^{q}}\left(a_{p q}(x) D^{p} u(x)\right)$

Le domaine $D(A)$ du triplet $A=\{v, H, a(u, v)\}$ est le sous-espace des fonctions $u$ de $\mathrm{H}^{\mathrm{m}}(\Omega)$ vérifiant :

$(4-4)\left\{\begin{array}{l}\text { i) } u \in V: A u \in L^{2}(\Omega) \\ \text { ii) } \int_{\Omega}(A u) \bar{v} d x=a(u, v) \text { pour tout } v \in v .\end{array}\right.$

Mais d'après la formule de Green (formelle), la seconde condition implique que : (4-5) $\int_{\Omega}(A u) \bar{v} d x-a(u, v)=\sum_{j=0}^{m-1}\left\langle s_{j} u, \gamma_{j} \bar{v}\right\rangle=0$ pour tout $v \varepsilon v$, où $s_{j}$ est un opérateur différentiel sur la frontière $r$ de $\Omega$, d'ordre $2 m-j-1$, où $\gamma_{j} u$ est la dérivée normale d'ordre $j$ de $u$. Ceci a un sens lorsque les crochets désignent la dualité entre les espaces $H^{-m+j+1 / 2}(\Gamma)$ et $H^{m-j-1 / 2}$ ( $\Gamma$ ) convenablement définis.

EXEMPLES.

Si $V=H_{0}^{m}(\Omega)$, la condition (4-5) est automatiquement vérifiée puisque $\gamma_{j} u=0$ pour $j=0, \ldots, m-1$. Le problème aux limites correspondant est 1. problème de Dirichlet associé à $A$.

Si $V=H^{m}(\Omega)$, la condition (4-5) signifie, qu'en un sens faible, $s_{j} u=0$ sur $\Gamma$ pour $f=0, \ldots, m-1$. Le problème aux limites correspondant est un problème de Neumann attaché à $A$.

On peut naturellement varier les exemples.

Remarquons aussi que les formes partielles :

(4-6) $a_{p q}(u, v)=\int_{\Omega} a_{p q}(x) D^{p} u D^{q} \bar{v} d x$ 
sont définies et continues sur $\mathrm{H}^{\mathrm{p}}(\Omega) \times \mathrm{H}^{\mathrm{q}}(\Omega)$ où $\mathrm{H}^{\mathrm{q}}(\Omega)=\mathrm{W}^{\mathrm{q}, 2}(\Omega)$.

LE PROBLEME DE LA COERCIVITE.

Ce problème est celui de la recherche des conditions suffisantes sur l'espace $V$ et les coefficients $a_{p q}(x)$ pour que 1 'une des conditions de coercivité $c_{1}$ ou $C_{2}$ suivantes soit réalisée.

Condition $c_{1}$.

(4-7) $\operatorname{Re} a(v, v) \geqslant c|| v \|^{2} \quad$ pour tout $v \in v ; c>0$

Condition $\mathrm{C}_{2}$.

$(4-8) \quad \operatorname{Re} \sum_{|p|,|q| \leqslant m} a_{p q}\left(u_{p}, u_{q}\right) \geqslant c \sum_{|p| \leqslant m}\left|p^{p} u_{p}\right|^{2} ; u_{p} \varepsilon H^{p}(\Omega)$.

Ce problème a été étudié par différents auteurs (nous renvoyons à J.L. LIONS [1] pour la bibliographie).

Si $m>1$, nous supposerons ces conditions satisfaites. Si $m=1$, elles résultent de :

$(4-9)\left\{\begin{array}{l}\text { i) } \operatorname{Re}\left[a_{i j}(x) \zeta_{i} \bar{\zeta}_{j} \geqslant c\left(\left|\zeta_{1}\right|^{2}+\ldots+\left|\zeta_{n}\right|^{2}\right) \text { p.p dans } \Omega .\right. \\ \text { ii) } \operatorname{Re} a_{0}(x) \geqslant c \quad p \cdot p \text { dans } \Omega .\end{array}\right.$

où $a_{i j}(x)=a_{e_{i} e_{j}}(x)$ et $a_{0}(x)=a_{0,0}(x)$.

Considérons maintenant la solution du

PROBLEME P .

Etant donné $\mathbf{f}$ dans $\mathrm{L}^{2}(\Omega)$, chercher $\mathbf{u}$ dans $\mathbf{v}$ vêrifiant :

(4-10) $|p|,|q| \leqslant m ~ \int_{\Omega} a_{p q}(x) D^{p} u D^{q} \bar{v} d x=\int_{\Omega} f \cdot \bar{v} d x$ pour tout $v \in v$.

La solution du problème $P$ est aussi celle du

PROBLEME $\mathrm{P}^{\prime}$.

Etant donné $\mathbf{f}$ dans $\mathrm{L}^{2}(\Omega)$, chercher $\mathrm{u}$ dans $\mathrm{V}$ verifiant : 


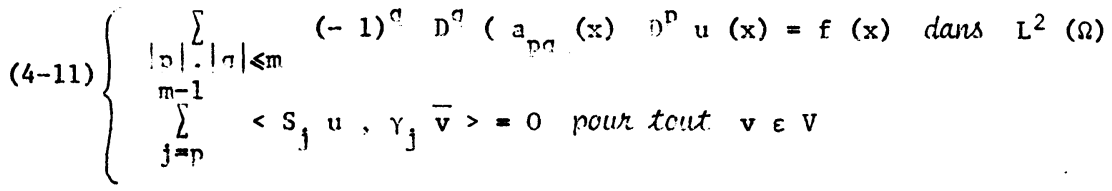

Si Ins conditions de coercivite sont satisfaites, cette solution existe et est unique.

4-2. APPROXIMUTION DES SOLUTIONS DU PROBLEAF. DE NELITANN POUR DES OPEPATEURS ELLIPTIOUFS D'ORDRE $2 \mathrm{~m}$.

ITnus rouvons maintenant déduire du $\$ 3$ du chanitre I et du $\$ 3$ du chanitre II des théorèmes d'approximation de la solution $"$ du nroblème $P$.

Considérons. pour fixer les idées, le cas du problème de Neumann, c'est-à-dire le cas où $\mathrm{V}=\mathrm{H}^{\mathrm{m}}(\Omega)$.

THEOREME 4-1.

Supposons tout d'abord que la condition de coercivité $C_{1}$ soit satisfaite. Si $L \geqslant(m)$. les solutions $u_{h}$ de l'équation :

(4-12) $\sum_{|p|,|q| \leqslant m} \int_{\Omega} a_{p q}(x) D^{p} p_{h}^{L} u_{h} D^{q} p_{h}^{L} \bar{v}_{h} d x=\int_{\Omega} f(x) p_{h}^{L} \bar{v}_{h} d x$ où $\mathrm{v}_{\mathrm{h}}$ parcourt l'espace $\mathrm{w}_{\mathrm{h}}^{\mathrm{m}, 2}(\Omega: \mathrm{L})$, convergent vers la solution $\mathrm{u}$ du problème $\mathrm{P}$ dans $\mathrm{w}^{\mathrm{m}, \mathrm{P}}(\Omega)$.

Si la solution u appartient $\pi H^{\ell}(\Omega)(\ell \geqslant m)$. si $L \geqslant \ell$ et si :

$$
\gamma_{\ell}^{m}(h)=\sup _{u}\|u\|_{\ell}^{-1}\left\|u-p_{h, \Omega}^{L}: r_{h} u\right\|_{m}
$$

alors

(4-13) $\quad|| u-p_{h, \Omega}^{L} \quad u_{h} \|_{m} \leqslant M r_{\dot{\ell}}^{m}(h)$

Supposons que la condition de coercivite. $C_{2}$ soit satisfaite. $S i L_{q}=L_{0}+q$, les solutions $u_{h}$ de l'équation:

$$
|p|,|q| \leqslant m=\int_{\Omega}{ }_{p q}(x) D^{P} p_{h}^{L_{p}} u_{h_{0}} \overline{D^{q} p_{h}^{L_{q}} v_{h}} d x=\int_{\Omega} f(x) \overline{p_{h}^{L_{o}} v_{h}} d x
$$

oü $v_{h}$ parcourt l'espace $\mathrm{W}_{h}^{\mathrm{m}, 2}(\Omega ; \overrightarrow{\mathrm{L}})$, convergent vers la solution $u$ au scns suivant : 
(4-15) $\lim _{h=0} \sum_{|p| \leqslant m}\left|D^{P} p_{h, \Omega}^{L_{p}} u_{h}-u\right|^{2}=0$

REMARQUE 4-1.

Nous montrerons dans un prochain travail qu'il existe une restriction $r_{h}$ telle que :

(4-16) $\quad \gamma_{\ell}^{m}(h) \leqslant c h^{\ell-m}$

ce qui montre que 1 'erreur décroit en fonction du choix de L. ( cf. AUBIN [3] ).

Lorsque $D(A)$ est contenu dans $H^{2 m}(\Omega), \gamma_{*}(h)$ est de l'ordre de $h^{m}$ et la norme dans $\mathrm{H}=L^{2}(\Omega) \quad 1$ 'erreur $u-p_{h} u_{h}$ est majorée par :

(4-17) $\left|u-p_{h} u_{h}\right| \leqslant c h^{\ell}$.

(cf. $\$ 3, n^{\circ}$ 3-3 du chapitre I).

Lorsque la condition $C_{2}$ est satisfaite, en prenant $K_{0}=0$, nous obtenons le problème approché suivant :

(4-18) $|p|,|q| \leqslant m ~ \int_{\Omega} a_{p q}(x) p_{h}^{o} \nabla_{h}^{p} \quad u_{h} \quad p_{h}^{o} \nabla_{h}^{q} \bar{v}_{h} d x=\int_{\Omega} f(x) p_{h}^{o} \bar{v}_{h} d x$ qui a été étudié par J. CEA [1].

Le théorème résulte du théorème $3-3$ du $n^{\circ} 3-3$ du $\$ 3$, chapitre $I$, du théorème 3-4 du $n^{\circ} 3-4$ du $\$ 3$, chapitre $I$, des théorèmes $3-4$ et 3-5 du $n^{\circ} 3-3$ du $\$ 3$, chapitre II.

Les problèmes approchés sont de la forme :

(4-19) $\quad A_{h} u_{h}=\sum_{|p|,|q| \leqslant m} A_{p, q, h}^{L_{p}, L_{q}} \quad u_{h}=f_{h}$

où la matrice $A_{p, q, h}^{L_{p}, L_{q}}$ est associée à la forme sesquilinéaire :

(4-20) $\int_{\Omega} a_{p q}(x) D^{p} p_{h}^{L_{p}} \quad u_{h} \quad D^{q} \quad p_{h}^{L_{q}} \quad \bar{v}_{h} d x$.

Nous allons maintenant calculer les coefficients $\underset{\mathrm{p}, \mathrm{q}, \mathrm{h}}{\mathrm{L}_{\mathrm{p}}, \mathrm{L}_{\mathrm{q}}}(\alpha, \beta)$ de la matrice $A_{p, q, h}^{L_{p}, L_{q}}\left(\right.$ où $\alpha, B \in R_{h}^{\vec{L}}(\Omega)$ )

Rappelons que : $\left(\$ 2, \mathrm{n}^{\circ} 2-1\right)$

(4-21) $a_{L_{p}}(k, l)=\left\{\begin{array}{l}\frac{1}{\left(L_{p}-l\right) !} \sum_{r \leqslant k}(-1)^{|r|} c_{L_{p}+(1)}^{r}(k-r)^{L_{p}-l} \text { si } 0 \leqslant k \leqslant L_{p} \\ 0 \text { dans le cas contraire. }\end{array}\right.$ 
Posons :

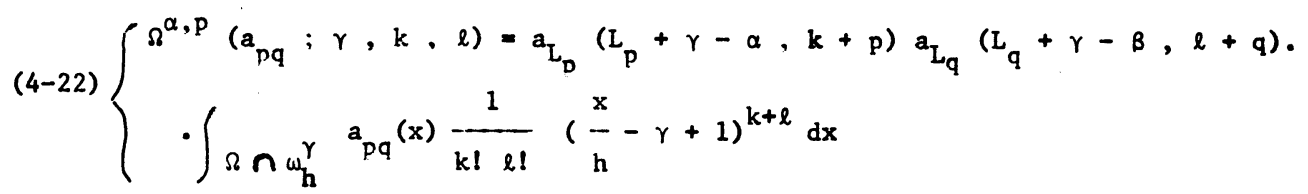

THEOREME 4-2.

Le terne génēral $a_{p, q, h}^{L_{p}, L_{q}}(\alpha, B)$ de la matrice $\underset{p, q, h}{L_{p}, L_{q}}$ est égal à :

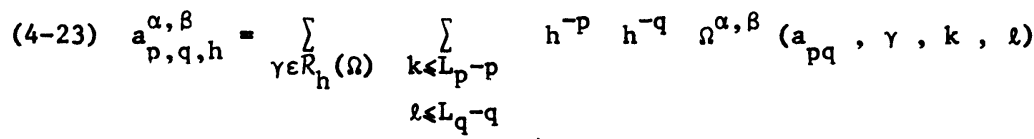
earsque $\alpha, B$ appartiennent $\bar{a} \vec{R}_{h}(\Omega)$.

En effet, 11 est clair que quand $\alpha, B \in R_{h}^{L}(\Omega)$, le terme général de la matrice est égal à :

$$
\underset{p, q, h}{L_{p}, L_{q}}(\alpha, \beta)=\int_{\Omega} a_{p q}(x) D^{p} \quad x_{L_{p}+(1), h}^{\alpha} D^{q} x_{L_{q}+(1), h}^{\beta} d x .
$$$$
\text { Nous explicitons cette expression. Puisque }\left(\$ 2, n^{\bullet} 2-1\right)
$$$$
x_{L_{p}}+(1)=\sum_{r \leqslant L_{p}} \psi_{L_{p}}^{r}\left(x+L_{p}-r\right) \cdot x^{-L_{p}+r}(x)
$$

On en déduit, en posant $\gamma=-L_{p}+r+\alpha=-L_{q}+s+B$, que

$$
\underset{p, q, h}{a_{p}, L_{q}}(\alpha, \beta)=\sum_{\gamma} \int_{\Omega} a_{p q}(x) D^{p} \cdot \psi_{L_{p}}^{L_{p}+\gamma-\alpha}\left(\frac{x}{h}-\gamma\right) D^{q}{ }_{L_{q}}^{L_{q}+\gamma-\beta}\left(\frac{x}{h}-\gamma\right) d x
$$

Puisque $\left(\$ 2, n^{\circ} 2-1\right)$ :

$D^{p} \quad \psi_{L_{p}}\left(\frac{x}{h}-\gamma\right)=h^{-p} \sum_{k \leqslant L_{p}-p} a_{L_{p}}(x, k+p) \frac{1}{k !}\left(\frac{x}{h}-\gamma+1\right)^{k}$

Il résulte de ces inégalités l'identité (4-23) .

CONSEQUENCES.

D'après (4-21), les indices $\gamma$ de $(4-23)$ vérifient en fait $\alpha-L_{p} \leqslant \gamma \leqslant \alpha$; B. $-L_{q} \leqslant \gamma \leqslant B$.

Si $l^{\prime}$ on pose $\beta=\alpha-\delta$, les coefficients $\underset{p, q, h}{L_{p}, L_{q}}(\alpha, \alpha-\delta)$ sont nuls si pour un indice $i$ au moins, on $a$ :

$$
\delta_{i}>L_{P_{i}} \text { ou } \delta_{1}<-L_{q_{1}}
$$


Il en résulte que le nombre maximun de diagonales non nulles àe la matrice $A_{\mathrm{p}, q, \mathrm{~h}}^{\mathrm{L}_{\mathrm{p}}, \mathrm{L}_{\mathrm{q}}}$ est égal à $\prod_{i=1}^{n}\left(\mathrm{~L}_{\mathrm{P}_{i}}+\mathrm{L}_{\mathrm{q}_{i}}+1\right)$

Les conditions aux limites interviennent dans les expressions des coefficients $\underset{p, q, h}{L_{p}, L_{q}}(\alpha, \beta)$ lorsque $l^{\prime}$ un des deux indices $\alpha$ ou $\beta$ appartient au réseau $R_{h}^{\vec{K}}(\Gamma)$. En effet, dans ce cas, $I^{\prime}$ un des pavés $\alpha-L_{p} \leqslant \gamma \leqslant \alpha$ ou $B-L_{q} \leqslant \gamma \leqslant \beta$ n'est pas contenu dans $R_{h}(\Omega)$.

Puisque le problème de la coercivité de l'opérateur A est supposé résolu, la matrice $A_{h}=\sum A_{p, q, h}^{L_{p}, L_{q}}$ est définie positive. Si 1'opérateur A est hermitien, 1a matrice $A_{h}$ est hermitienne. Si $q \neq 0$, alors $\sum_{B} \underset{p, q, h}{a} L_{p}, L_{q}(\alpha, B)=0$.

En effet :

$$
\sum_{\beta} \underset{p, q, h}{L_{p}, L_{q}}(\alpha, B)=\int_{\Omega} a_{p q}(x) \quad D^{p} x_{L_{p}+(1), h}^{\alpha} D^{q} \sum_{\beta} x_{L_{q}+(1), h}^{\beta} d x=0
$$

REMARQUE $4-2$.

$$
\begin{array}{r}
\text { Considérons le cas particulier où } m=1 \text { et où } \\
a_{h}\left(u_{h}, v_{h}\right)=a\left(p_{h}^{l} u_{h}, p_{h}^{l} v_{h}\right)(L=(1))
\end{array}
$$

Supposons que $\mathrm{f}$ appartienne à $L^{2}(\Omega)$. D'après le théorème 4-1, on en déduit que

$$
\begin{gathered}
\left\|u-p_{h, \Omega}^{1} u_{h}\right\|_{1} \leqslant c h \\
\text { puisque } \quad\left\|u-p_{h, \Omega}^{1} r_{h} u\right\|_{1} \leqslant c h\|u\|_{2}
\end{gathered}
$$

En prenant le même schéma approché, Y. DEMIANOVITCH [1] a démontré, par d'autres méthodes, que :

$$
\left\|u-p_{h, \Omega}^{1} u_{h}\right\|_{1} \leqslant c \sqrt{h} \text {. }
$$

4-3. APPROXIMATION DES SOLUTIONS DES PROBLEMES AUX LIMITES POUR DES OPERATEURS DIFFERENTIELS NON LINEAIRES.

EXEMPLE 4-1.

Soit $\Omega$ un ouvert borné de $R^{n}$. Prenons pour espace $V$ l'espace de Banach $\mathrm{W}_{\mathrm{m}}^{\mathrm{m}, \mathrm{P}}(\Omega)$ et pour forme $\mathrm{a}(\mathrm{u} ; \mathrm{v})$ la forme : 
(4-24) $\quad a(u ; v)=\sum_{i=1}^{n} \int_{\Omega}\left|D_{i} u\right|^{\rho} b_{i} u \cdot D_{i} v \cdot d x: \rho>0$.

Cette forme est définie sur $V \times V$ si

$P=\rho+2$. Alors $P^{\prime}=\frac{\rho+2}{\rho+1}=\frac{P}{\rho+1} \quad ; \quad \frac{1}{P}+\frac{1}{P^{\prime}}=1$

Il est clair que dans ce cas, les hypothèses de la proposition 3-4 du $n^{\circ} 3-5$. $\$ 3$, chapitre I sont satisfaites. L'opérateur non linéaire A de $\mathrm{W}_{0}^{1, P}(\Omega)$ dans $\mathrm{W}^{1, \mathrm{P}^{\prime}}(\Omega)$ défini par :

(4-25) $\quad A(u)=-\sum_{i=1}^{n} D_{i}\left(\left|D_{i} u\right|^{p} D_{i} u\right)$

est borné, coercif, monotone et continu des droites de $V$ dans $V^{\prime}$ faible, donc surjectif.

On déduit de la proposition $3-4$ du $\$ 3$ du chapitre $I$ et des résultats du $n^{\circ} 3-2$ du $\$ 3$ du chapitre II la :

PROPOSITION 4-1.

Si $L \geqslant(1)$, les solutions $u_{h}$ du problème non linéaire:

$$
\sum_{i=1}^{n} \int_{\Omega}\left|D_{i} p_{h}^{L} u_{h}\right|^{\rho} \quad D_{i} p_{h}^{L} u_{h} \cdot D_{i} p_{h}^{L} v_{h} d x=\left(f, p_{h}^{L} v_{h}\right)
$$

pour tout $v_{h}$ de $w_{0, h}^{m, P}(\Omega ; L)$, oil $\mathrm{f}$ appartient $\bar{a} L^{P^{\prime}}(\Omega)$, conversent iaiblement vers une solution $\mathrm{u}$ de l'équation $\mathrm{A}(\mathrm{u})=\mathrm{f} . \mathrm{Si} \mathrm{L}_{\mathrm{o}}=0, \mathrm{~L}_{\mathbf{i}} \geqslant \mathrm{e}_{\mathbf{i}}$ $(i=1, \ldots, n)$ et $\vec{L}=\left(L_{0}, L_{1}, \ldots, L_{n}\right)$, les solutions $u_{h}$ de $l^{\prime} e_{q u a-}$ tion variationnelse:

(4-27) $\sum_{i=1}^{n} \int_{\Omega}\left|D_{i} p_{h}^{L_{i}} u_{h}\right|^{\rho} \quad D_{i} p_{h}^{L_{i}} u_{h} D_{i} p_{h}^{L_{i}} v_{h} d x=\left(f, p_{h}^{L_{o}} v_{h}\right)$ pour tout $v_{h}$ de $\mathrm{w}_{\mathrm{o}, \mathrm{h}}^{\mathrm{m}, \mathrm{h}}(\Omega, \overrightarrow{\mathrm{L}})$ convergent vers une solution $\mathrm{u}$ de l'équation $\mathrm{A}(\mathrm{u})=\mathrm{f}$ au sens suivant :

$$
\begin{aligned}
& \left\{\begin{array}{c}
D_{i} p_{h}^{L_{i}} u_{h} \text { converge vers } D_{i} u \text { dans } L^{P}(\Omega) \text { faible. } \\
p_{h}^{L_{0}} u_{h} \text { converge vers } u \text { dans } L^{P}(\Omega) \text { faible. }
\end{array}\right. \\
& \text { En particulier, si } L_{0}=0 \text { et } L_{i}=e_{i}, 1^{\prime} \text { équation }(4-27) s^{\prime} \text { écrit : } \\
& \sum_{\alpha \varepsilon R_{h}^{(1)}(\Omega ; \Gamma)}\left|\nabla_{h_{i}} u_{h}^{\alpha}\right|^{\rho} \nabla_{h_{i}} u_{h}^{\alpha} \cdot \nabla_{h_{i}} v_{h}^{\alpha}=h^{1 / 2} \sum_{\alpha \varepsilon R_{h}^{(1)}(\Omega ; \Gamma)}\left(\int f x_{h}^{\alpha} d x\right) v_{h}^{\alpha}
\end{aligned}
$$


EXEMPLE 4-2.

Soit toujours $\Omega$ un ouvert borné de $R^{n}$, de frontière $\Gamma$ suffisamment régu1ière.

On prend pour espace $V$ le sous-espace fermé des fonctions $v$ de $B^{1}(\Omega)$ dont la trace $\gamma_{0} v$ appartient à $L^{P}(\Gamma)$. On prend pour forme $a(u ; v)$ la forme (4-28) $a(u: v)=\int_{\Omega} u \cdot v d x+\sum_{i=1}^{n} \int_{\Omega} D_{i} u \cdot D_{i} v d x+\int_{\Gamma}|u|^{P-2} u \cdot v d \sigma$ où do est une mesure superficielle sur $\Gamma$.

L'espace $V$ est un espace de Banach pour la norme :

$$
\|v\|=\left.|| v\right|_{H^{1}(\Omega)}+\left|\gamma_{0} v\right|_{L^{P}(r)}
$$

Les hypothèses de $1 a$ proposition $3-4$ du $n^{\circ} 3-5$ du $\$ 3$ du chapitre I sont satisfaites.

Soit $f \in L^{2}(\Omega), g \in L^{P^{\prime}}(\Gamma)$. Le couple $\{f, g\}$ définit une forme linéaire sur $\mathrm{V}$.

On considère le :

PROBLEME P :

Chercher $u$ dans $v$ tel que $a(u ; v)=\int_{\Omega} f v d x+\int_{\Gamma} g v \cdot d x$

Une solution u du problème $\mathrm{P}$ vérifie :

(4-29) $\left\{\begin{array}{l}-\Delta u+u=f \text { dans } L^{P^{\prime}(\Omega)} \\ \left.|u|^{P-2} u \cdot\right|_{\Gamma}=g \text { sur } \Gamma\end{array}\right.$

On déduit de la proposition 3-4 du chapitre $I$ et des résultats du $n^{\circ} 3-3$ du $\$ 3$ du chapitre II la :

PROPOSITION 4-2.

Si $L \geqslant 1$, les solutions $u_{h}$ de l'équation variationnelle

(4-30) $\left\{\begin{array}{l}\int_{\Omega} p_{h}^{L} u_{h} p_{h}^{L} v_{h} d x+\sum_{i=1}^{n} \int_{\Omega} D_{i} p_{h}^{L} u_{h} p_{i} p_{h}^{L} v_{h} d x+ \\ \int_{\Gamma}\left|p_{h}^{L} u_{h}\right|^{p-2} p_{h}^{L} u_{h} p_{h}^{L} v_{h} d \sigma=\int_{\Omega} f p_{h}^{L} v_{h} d x+\int_{\Gamma} g p_{h}^{L} v_{h} d \sigma\end{array}\right.$ 
pour tout $v_{h}$ de $w_{h}^{m, P}(\Omega, L)$, convernent faiblement vers une solution $u d u$ problènie $\mathrm{P}$.

Si $L_{i}=L_{0}+e_{i}(I=1 \ldots ., n)$ les solutions $u_{h}$ de l'équation variationnelle:

$(4-31)\left\{\begin{array}{l}\sum_{i=1}^{n} \int_{\Omega} p_{i} p_{h}^{L_{1}} u_{h} p_{i} p_{h}^{L_{1}} v_{h} d x+\int_{\Omega} p_{h}^{L_{o}} u_{h} p_{h}^{L_{o}} v_{h} d x+ \\ \int \Gamma\left|p_{h}^{L_{o}} u_{h}\right|^{P-2} \cdot p_{h}^{L_{o}} u_{h} \cdot p_{h}^{L_{o} o} v_{h} d \sigma=\int_{\Omega} f p_{h}^{L_{o}} v_{h} d x+\int_{\Gamma} f p_{h}^{L_{o}} v_{h} d \sigma\end{array}\right.$ pour tout $v_{h}$ de $R_{h}^{\vec{L}}(\Omega)$, convergent vers une solution $u$ du problème $P$ au seirs suivant :

$\left\{\begin{array}{l}r_{h}^{L_{0}} u_{h} \text { converae vers } u \text { dans } L^{2}(\Omega) \text { faible; } \\ r_{0} r_{h}^{L_{0}} u_{h} \text { converae vers } r_{0} u \text { dans } L^{P}(\Gamma) \text { faible; } \\ D_{i} r_{h}^{L_{i}} u_{h} \text { converae vers } D_{i} u \text { dans } L^{2}(\Omega) \text { faible. }\end{array}\right.$

Si l'on nrend $L_{0}=0$. si $e_{h}^{\alpha}$ est la suite qui vaut 1 au point a et 0 ailleurs, 1'éqquation 4-31 revient à résoudre le système (4-32), (4-33) :

(4-32) $\sum_{i=1}^{n} \nabla_{h_{i}} u_{h}^{\alpha} \nabla_{h_{1}} e_{h}^{\alpha}+u_{h}^{\alpha} e_{h}^{\alpha}=h^{1 / 2}\left(\int f x_{h}^{\alpha} d x\right) e_{h}^{\alpha}: \alpha \varepsilon \vec{R}_{h}(\Omega, \Gamma)$

$(4-33)\left\{\begin{array}{l}\sum_{i=1}^{h} \nabla_{h}^{i} u_{h}^{\alpha} \cdot \Omega_{h}^{\alpha} \cdot \nabla_{h_{i}} e_{h}^{\alpha}+\Omega_{h}^{\alpha} u_{h}^{\alpha} e_{h}^{\alpha}+\Gamma_{h}^{\alpha}\left|u_{h}^{\alpha}\right|^{p-2} u_{h}^{\alpha} e_{h}^{\alpha}= \\ =h^{1 / 2}\left(\int_{\Omega} f x_{h}^{\alpha} d x+\int_{\Gamma} g x_{h}^{\alpha} d \sigma\right) e_{h}^{\alpha} ; \alpha \varepsilon R_{h}^{1}(\Gamma)\end{array}\right.$

où $\Omega_{h}^{\alpha}=h^{-1}$ mes $\left(\Omega \cap \omega_{h}^{\alpha}\right)$ et $\Gamma_{h}^{\alpha}=h^{-1}$ mes $\left(r \cap \omega_{h}^{\alpha}\right)$

Remarquons enfin que $s i v \in H^{l}(\Omega)$, alors $\gamma_{0} v \in H^{1 / 2}(r)$.

Si $P \leqslant \frac{2 n}{n-1} \cdot H^{1}(\Gamma)$ est contenu dans $L^{P}(r)$ d'après le théorème de Sobolev fractionnaire et $\mathcal{L}^{\prime}$ espace $V$ est égal à $\mathbf{H}^{1}(\Omega)$.

EXEYPLE 4-3.

Donnons $n$ fonctions $a_{i}(x, u)$ définies sur $\Omega \times R$ vérifiant, si $P \geqslant 2$ 
$(4-34)\left\{\begin{array}{l}\text { i) }\left|a_{i}(x, u)\right| \leqslant M|u|^{p-1}: \text { nour presque tout } x \text { de } \Omega \\ \text { ii) } a_{i}(x . u) u \geqslant c|u|^{p} \\ \text { iii) } a_{i}(x \cdot u)-a_{i}(x \cdot v) \geqslant c|u-v|^{p-2}(u-v)\end{array}\right.$

Considérons alors la forme a $(\mathrm{u}: \mathrm{v})$ suivante sur $\mathrm{w}_{0}^{1, P}()$ :

(4-35) a ( u; v ) $=\sum_{i=1}^{n} \int_{\Omega} a_{i}\left(x: D_{i} u\right) D_{i} v d x=\sum_{i=1}^{n} a_{i}(u, v)$

rîâce à l'inégalité de Poincaré. les hypothèses suivantes sont satisfaites :

$(4-36)\left\{\begin{array}{l}\text { i) } A(u) \text { est borné et continu des droites de } V \text { dans } V^{\prime} \text { faible } \\ \text { ii) } a_{i}\left(u_{i}: v_{i}\right) \geqslant c\|u\|_{i . P}^{p} \\ \text { iii) } \sum_{i=1}^{n} \sigma_{i}\left(u_{i}, v_{i}\right) \geqslant c \sum_{i=1}^{n}\left\|u_{i}-v_{i}\right\|_{i, P}^{P}\end{array}\right.$

cì $\left\|u_{i}\right\|_{i, p}=\left|D_{i} u_{i}\right|_{p}$

Si $f \in L^{P^{\prime}}(\Omega)$. on déduit des résultats du $\$ 3$ du chapitre $I$ qu'il existe une solution uniq̣ue $u$ de $W_{0}^{1, P}(\Omega)$ telle que

(4-37) $\left\{\begin{array}{l}A(u)=-\sum_{i=1}^{n} D_{i} a_{i}\left(x \cdot D_{i} u\right)=f \text { dans } L^{P^{\prime}}(\Omega) \\ \left.u\right|_{\Gamma}=0 .\end{array}\right.$

On en déduit alors la :

PROPOSITION 4-3.

Si $L \geqslant 1$, les soiutions $u_{h}$ des equations variationneiles :

(4-38) $\quad \sum_{i=1}^{n} \int_{\Omega} a_{i}\left(x: p_{h}^{L} u_{h}\right) p_{h}^{L} v_{h} d x=\int_{\Omega} f p_{h}^{L} v_{h} d x$

nour tout $v_{h}$ de $w_{0 . h}^{1 . P}(\Omega: L)$ converaent fortement vers la soiution $u$ de. (4-37) et: :

(4-39) $\quad\left|l u-p_{h}^{L} u_{h}\left\|_{1 . P} \leqslant k|| u-p_{h} r_{h} u\right\|_{1, P}^{1 / p}\right.$

Si $\overrightarrow{\mathrm{L}}=\left(\mathrm{L}_{i}\right)_{0 \leqslant i \leqslant n}$. avec $\mathrm{L}_{i} \geqslant e_{i}$. ies solutions $u_{h}$ de: 
(4-40) $\sum_{i=1}^{n} \int_{\Omega} a_{i}\left(x: p_{h}^{L_{i}} u_{h}\right) p_{h}^{L_{i}} v_{h} d x=\int_{\Omega} f p_{h}^{L_{O}} v_{h} d x$

converaent vers $\mathrm{u}$ an seis suivant :

(4-41) $\lim _{h=0}\left|\mathrm{p}_{i} p_{h}^{L_{i}} u_{h}-u\right|_{L} P(\Omega)=0: \lim _{h=0}\left|p_{h}^{L_{o}} u_{h}-u\right|_{L} P(\Omega)=0$

4-4. APPROXIMATION DES SOLUTIONS DE PROBLEMES AUX LIMITES UNILATERAUX - CALCUL D'UNE CAPACITE.

Nous donnons maintenant des exemples d'approximations de solutions d'inéruations variationnelles, étudiées au $\$ 4$ du chapitre I .

EXEMPLI: 4-4. Problèmes aux limites unilatéraux.

Prenons pour espace $V$ l'espace $H^{1}(\Omega)$. pour cône convexe $K$ l'ensemble des fonctions $v$ de $V$ de trace positive et pour forme a ( $u, v)$ la forme : (4-42) a ( u . v ) $=\sum_{i=1}^{n} \int_{\Omega} a_{i j}(x) D_{i} u \cdot D_{i} v d x+\int_{\Omega} a_{0}(x) u \cdot v d x$ où $a_{i j}(x) \in L^{\infty}(\Omega), a_{0}(x) \in L^{\infty}(\Omega)$ vérifient : (4-43) $\sum_{j, i=1}^{n} a_{i j}(x) \zeta_{i} \zeta_{j} \geqslant c|r|^{2} ; a_{o}(x) \geqslant c \quad p . p$ sur $\Omega$.

Considérons la solution $\mathrm{u}$ du Problème $\mathrm{P}$ : Chercher $\mathrm{u}$ dans $\mathrm{K}$ telle que $a(u: u-v) \leqslant(f, u-v)$ pour tout $v \in K: f \in L^{2}(\Omega)$ Elle vêrifie :

$$
(4-44)\left\{\begin{array}{l}
\text { i) }-\sum_{i, j=1}^{n} D_{j}\left(a_{i j}(x) D_{i} u\right)+a_{0}(x) u=f \text { dans } L^{2}(\Omega) \\
\text { ii) }\left.u\right|_{\Gamma} \geqslant 0 \quad:\left.\quad \frac{\partial u}{\partial v_{A}}\right|_{\Gamma} \geqslant 0 \\
\text { iii) }\left.u \frac{\partial u}{\partial v_{A}}\right|_{\Gamma}=0 .
\end{array}\right.
$$

On déduit du corollaire 4-2 du $\$ 4$ du chapitre I et du théorème $3-7$ du n $n^{\circ}$ 3-4 du $\$ 3$ du chapitre II le : 
THEOREME 4-3.

Si $L \geqslant(1)$, les solutions $u_{h}$ de l'inéquation variationnelle :

$(4-45)\left\{\begin{array}{l}\sum_{1, j=1}^{n} \int_{\Omega} a_{i j}(x) D_{i} p_{h}^{L} u_{h}\left(D_{j} p_{h}^{L}\left(u_{h}-v_{h}\right)\right) d x+\int_{\Omega} a_{o}(x) p_{h}^{L} u_{h} p_{h}^{L}\left(u_{h}-v_{h}\right) d x \\ \leqslant \int_{\Omega} f(x) p_{h}^{L}\left(u_{h}-v_{h}\right) d x \text { pour tout } v_{h} \varepsilon K_{h} .\end{array}\right.$ convergent vers $u$ dans $H^{1}(\Omega)$ fort, et :

(4-46) $\quad\left\|u-p_{h}^{L} u_{h}\right\| \leqslant M\left\|u-p_{h}^{L} r_{h} u\right\|^{1 / 2}$

Si $L_{i}=L_{0}+e_{i}, \quad \vec{L}=\left(L_{i}\right)_{0 \leqslant i \leqslant n}$, les solutions $u_{h}$ de l'inéquation variationnelle:

$(4-47)\left\{\begin{array}{l}\sum_{i, j=1}^{n} \int_{\Omega} a_{i j}(x) D_{i} p_{h}^{L_{1}} u_{h}\left(D_{j} p_{h}^{L_{j}}\left(u_{h}-v_{h}\right)\right)+\int_{\Omega} a_{o}(x) p_{h}^{L_{0}} u_{h} p_{h}^{L_{0}}\left(u_{h}-v_{h}\right) d x \\ \leqslant \int_{\Omega} f(x) p_{h}^{L_{0}}\left(u_{h}-v_{h}\right) d x \text { pour tout } v_{h} \varepsilon k_{h} .\end{array}\right.$ convergent vers $\mathrm{u}$ au sens suivant :

(4-48) $\lim _{h=0}\left|D_{i} u-D_{i} p_{h}^{L_{i}} u_{h}\right|=0: \lim _{h=0}\left|u-\eta_{h}^{L_{0}} u_{h}\right|=0$.

EXEMPLE 4-4. Calcul d'une capacité.

Prenons pour espace $V$ l'espace $H_{0}^{l}(\Omega)$, pour convexe fermé $K \quad$ l'ensemble des fonction $u$ plus grandes que 1 sur $E$. (cf. Exemple $3-1$ du $n^{\circ} 3-4$ du $\$ 3$ du chapitre II), pour forme $a(u ; v)$ la forme

(4-49) a ( u ; v ) $=\sum_{i, j=1}^{n} \int_{\Omega} a_{i j}(x) D_{i} u D_{j} v d x$

où les coefficients $a_{i j}(x)$ vérifient $(4-43)$.

Considérons la solution du PROBLEME $\mathrm{P}$ : Chercher $\mathrm{u}$ dans $\mathrm{K}$ telle que :

(4-50) a $(\mathrm{u} ; \mathrm{u}-\mathrm{v}) \leqslant 0$ pour tout $\mathrm{v}$ de $\mathrm{K}$.

La solution $\mathbf{u}$ du problème $\mathbf{P}$ est égale à 1 sur $\mathbf{E}$ et la distribution $\mathbf{v} \rightarrow \mathbf{a}(\mathrm{u}, \mathrm{v})$ est une mesure positive portée par la frontière de $\mathrm{E}$, appelée mesure capacitaire de $\mathrm{E}$ par rapport à la forme a ( $\mathrm{u}, \mathrm{v})$. 
PROPOSITION 4-4.

Si $\vec{L}=\left(L_{i}\right)_{0 \leqslant i \leqslant n}$, avec $L_{i} \geqslant e_{i}$, les solutions $u_{h}$ de l'inéquation variationnclle:

(4-51) $\quad \sum_{1, j=1}^{n} \int_{\Omega} a_{i j} D_{i} p_{h}^{L_{i}} \quad u_{h} D_{j} p_{h}^{L_{j}}\left(u_{h}-v_{h}\right) d x \leqslant 0$

nour tout $v_{h}$ de $k_{h}$, convergent vers $u$ au sens suivant :

$$
\begin{aligned}
& \operatorname{Lim}_{h=0}\left|D_{1} p_{h}^{L_{i}} u_{h}-D_{i} u\right|=0 ; \lim _{h=0}\left|p_{h}^{L_{0}} u_{h}-u\right|=0 . \\
& \text { Si } L_{i}=L \geqslant(1) \text {, alors }
\end{aligned}
$$

(4-52) $\quad\left\|u-p_{h}^{L} u_{h}\right\| \leqslant M\left\|u-p_{h}^{L} r_{h} u\right\|^{1 / 2}$

\$5. APPROXIMATION DES SOLUTIONS DES EQUATIONS DIFFERENTIELLES-OPERATIONNELLES (II).

Nous poursuivons $1^{\prime}$ étude du $\$ 5$ du chapitre $I$, en étudiant au $n^{\bullet} 5-1$, la converģence forte des solutions du "schéma implicite" et de leurs dérivées par rapport à $t$. Nous appliquons ce résultat au $n^{\circ}$ 5-2 à l'étude des solutions du problème de Cauchy-Dirichlet pour les équations paraboliques. En uțlisant 1 'approximation des solutions du Lemme des Projections ( $n^{\circ} 3-6, \$ 3$, chapitre I), nous construisons au $n^{\circ}$ 5-3 des schémas d'approximation implicites à $2 L+1$ niveaux, dont certaines solutions convergent fortement.

5-1. CONVERGENCE FORTE DES SOLUTIONS DU SCHEMA EXPLICITE.

Faisons les hypothèses du théorème $5-1$ du $\$ 5$ du chapitre I . Nous avions alors approché la solution u du

PROBLEME P .

Chercher $u$ dans $w$ tel que :

$(5-1)\left\{\begin{array}{l}\text { 1) } \frac{d u}{d t}+A(t) u=f(t) \text { dans } L^{2}\left(0, T ; V^{\prime}\right)=V^{\prime} . \\ \text { ii) } u(0)=u_{0} \text { dans } H .\end{array}\right.$

par les solutions $u_{h} d u$ : 
PROBLEME $\mathrm{P}_{\mathrm{h}}$.

Chercher $u_{h}$ dans $w_{h}$ tel que:

$(5-2)\left\{\begin{array}{l}\text { 1) } \frac{d u_{h}}{d t}+A_{h}(t) u_{h}=f_{h}(t) \text { dans } L^{2}\left(0, T ; v_{h}^{\prime}\right)=V_{h}^{\prime} \cdot \\ \text { ii) } u_{h}(0)=u_{\text {oh }} \text { dans } v_{h} .\end{array}\right.$

Nous allons approcher les solutions $u_{h}$ des équations différentielles par des solutions $u_{h, k}$ d'équations algébriques et montrer la convergence forte des solutions $u_{h, k}$ vers 1 a solution $u$ du problème $P$ dans 1 'espace $W$ des fonctions $\mathrm{u}$ de $\mathrm{L}^{2}(0, \mathrm{~T} ; \mathrm{V})$ dont la dérivée $\mathrm{u}^{\prime}$ appartient $\mathrm{a} \mathrm{L}^{2}\left(0, T ; \mathrm{V}^{\prime}\right)$.

On se donne pour cela des approximations autoadjointes stables et convergentes $A_{k}\left(V_{h}\right)$ de l'espace $V_{h}=L^{2}\left(0, T ; V_{h}\right)$ :

$\left.(5-3) \quad A_{k}\left(v_{h}\right)=\left(v_{h}, v_{\zeta}, p_{k}, r_{k}^{*}\right) ; k \varepsilon\right] 0,1[; \zeta=(h, k)$

où :

(5-4) $R_{k}(0, T)=\left\{\mu \varepsilon N\right.$ tels que $\left.1 \leqslant \mu \leqslant T k^{-1}+1\right\}$

$(5-5)\left\{\begin{array}{l}V_{\zeta}=\ell^{2}\left(R_{k}(0, T) ; v_{h}\right) \text { est } I^{\prime} \text { espace des suites } u_{\zeta}=\left(u_{\zeta}^{\mu}\right) \text { défi- } \\ \text { nies sur } R_{k}(0, T) \text { à valeurs dans } v_{h} \text {. }\end{array}\right.$

(5-6) $\quad p_{k} u_{\zeta}=\sqrt{k} \sum_{\mu} u_{\zeta}^{\mu} x_{k}^{\mu}(t) ; r_{k}^{*} u_{h}=\left(\sqrt{k} \int_{0}^{T} u_{h}(t) x_{k}^{\mu}(t) d t\right)_{\mu}$

où $x_{k}^{\mu}(t)$ est égale à $\frac{1}{k} \times\left(\frac{t}{k}-\mu\right), x$ étant la fonction caractéristique de l'intervalle $(-1,0)$.

On associe aux approximations $A_{k}\left(v_{h}\right)$ et $A_{h}(v)$ les approximations autoadjointes de $V=L^{2}(O, T ; V)$ dans $V_{\zeta}$ :

$$
\text { 1) } \begin{aligned}
A_{\zeta}(V) & =\left(V, V_{\zeta}, p_{\zeta}=p_{k} p_{h}, r_{\zeta}^{*}=r_{k}^{*} r_{h}^{*}\right) \\
\text { 11) } A_{\zeta}\left(V^{\prime}\right) & =\left(V^{\prime}, V_{\zeta}^{\prime}, p_{\zeta}=p_{k} p_{h} ; r_{\zeta}^{*}=r_{k}^{*} r_{h}^{*}\right)
\end{aligned}
$$

où le produit scalaire sur $V_{\zeta}$ est défini par :

(5-8) $\quad\left(u_{\zeta}, v_{\zeta}\right)_{\zeta}=\int_{0}^{T}\left(p_{k} u_{\zeta}, p_{k} v_{\zeta}\right)_{h} d t=\sum_{\mu}\left(u_{\zeta}^{\mu}, v_{\zeta}^{\mu}\right)_{h}$ 
On introduit maintenant les op̣érateurs $A_{\zeta}$ définis par :

$(5-9)\left\{\begin{array}{l}a_{\zeta}\left(u_{\zeta}, v_{\zeta}\right)=\int_{0}^{T} a_{h}\left(t ; p_{k} u_{\zeta}, p_{k} v_{\zeta}\right) d t= \\ =\sum_{\nu} \int_{0}^{T} a_{h}\left(t ; u_{\zeta}^{\mu}, v_{\zeta}^{\mu}\right) x_{k}^{\mu} d t=\sum_{\mu}\left(A_{\zeta}^{\mu} u_{\zeta}^{\mu} \cdot v_{\zeta}^{\mu}\right)_{h} .\end{array}\right.$

et $f_{\zeta}=r_{k}^{*} f_{h}$ :

(5-10) $\quad\left(f_{\zeta}, v_{\zeta}\right)_{\zeta}=\int_{0}^{T}\left(f_{h}(t), p_{k} v_{\zeta}\right) d t:$ du :

Nous approchons alors les solutions $u_{h}$ du problème $P_{h}$ par les solutions $u_{\zeta}$

PROBLEME $\mathrm{P}_{\zeta} \cdot$

Chercher la suite $u_{\zeta}=\left(u_{\zeta}^{\mu}\right)$ définie sur $R_{k}(0, T)$ à valcurs dans $v_{h}$ verifiant :

$(5-11) \begin{cases}i) & \left(\nabla_{k} u_{\zeta}, v_{\zeta}\right)_{\zeta}+a_{\zeta}\left(u_{\zeta}, v_{\zeta}\right)=\left(f_{\zeta}, v_{\zeta}\right)_{\zeta} \text { pour tout } v_{\zeta} \varepsilon v_{\zeta} \\ \text { ii) } & u_{\zeta}^{1}=u_{o h}\end{cases}$

où $\left(\nabla_{k} u_{\zeta}\right)^{\mu}=k^{-1}\left(u_{\zeta}^{\mu+1}-u_{\zeta}^{\mu}\right)$

La suite $u_{\zeta}$ est bien définie : on obtient $u_{\zeta}^{\mu+1}$ à partir de $u_{\zeta}^{\mu}$ par :

(5-12) $\quad u_{\zeta}^{\mu+1}=u_{\zeta}^{\mu}-k A_{\zeta}^{\mu} u_{\zeta}^{\mu}+k f_{\zeta}^{\mu} ; \quad u_{\zeta}^{1}=u_{o h}$

L'espace $V_{h}$ étant de dimension finie, nous pouvons noser :

$(5-13) \quad s(h)=\sup _{u_{h}}\left\|u_{h}\right\|_{h}^{-1}\left|u_{h}\right|_{h}$

Rappelons que :

(5-14) $\left|a_{h}\left(t ; u_{h} \cdot v_{h}\right)\right| \leqslant\left. M|| u_{h}||_{h}|| v_{h}\right|_{h} ; \operatorname{Re} a_{h}\left(t ; u_{h}, v_{h}\right) \geqslant c|| u_{h}||_{h}^{2} \cdot$

D'après P.A. RAVIART [1] (chapitre I , théorème 3-1), les solutions ${ }_{\zeta}$ du problème ${ }_{\zeta}$ vérifient une inégalité de l'énergie :

THEOREME A .

Si $\zeta$ verifie la condition:

(5-15) $M^{2} k S(h)^{2} \leqslant 2 c(1-\delta) \therefore \delta>0$ arbitrairement petit, 
les solutions ' ${ }_{\zeta}$ vērifient l'inégalité de l'énerzie suivante :

(5-16) $\sup _{t}\left|p_{k} u_{\zeta}\right|_{h}^{2}+\int_{0}^{T}\left\|p_{k} u_{\zeta}\right\|_{h}^{2} d t \leqslant M\left(|| f_{\zeta} \|_{V^{\prime}}^{2}+\left|u_{o h}\right|_{h}^{2}\right)$

On obtient (5-16) en prenant dans (5-11) successivement $v_{\zeta}=u_{\zeta}$ et $v_{\zeta}=\nabla_{k} u_{\zeta}$ et en utilisant la relation :

$2 \operatorname{Re}\left(u, \nabla_{k} u\right)=\nabla_{k}|u|^{2}-k\left|\nabla_{k} u\right|^{2}$

Posons (cf. \$3) :

(5-17) $\quad \mathrm{p}_{\mathrm{k}}^{1}=\mathrm{x}_{\mathrm{k}} * \mathrm{p}_{\mathrm{k}}^{0} \quad ; \quad \mathrm{p}_{\zeta}^{1}=\mathrm{p}_{\mathrm{k}}^{1} \mathrm{p}_{\mathrm{h}}$.

Nous allons montrer le :

THEOREME 5-1.

Supposons que les approximations $A_{h}(V)$ soient autoadjointes, stables et convergentes, que:

(5-18) $\lim _{h=0}\left[\int_{0}^{T}\left\|p_{h} v_{h}\right\|_{h}^{2} d t\right]^{-1 / 2} \int_{0}^{T}\left|a_{h}\left(t ; r_{h}^{*} u, v_{h}\right)-a\left(t ; u, p_{h} v_{h}\right)\right| d t=0$

$(5-19) \begin{cases}\text { i) } & \lim _{h=0}\left|u_{o h}-r_{h}^{*} u_{0}\right|_{h}=0 \\ \text { ii) } & \lim _{h=0}\left[\int_{0}^{T}\left|p_{h} v_{h}\right|_{h}^{2} d t\right]^{-1 / 2} \int_{0}^{T}\left|\left(f_{h}(t), v_{h}\right)_{h}-\left(f(t), p_{h} v_{h}\right)\right| d t=0\end{cases}$

et que (5-15) a lieu. Les solutions $u_{\zeta}$ du problème ${ }_{{ }_{\zeta}}$ convergent alors vers la solution $u$ du problème $P$ au sens suivant :

(5-20) $\lim _{h=0} \int_{0}^{T}\left\{\left\|u-p_{\zeta}^{1} u_{\zeta}\right\|^{2}+\left\|u^{\prime}-\frac{d}{d t} p_{\zeta}^{1} u_{\zeta}\right\|_{\varkappa}^{2}\right\} d t=0$

Il en résulte en particulier que :

(5-21) $\lim _{h=0} \sup _{t \in[0, T]}\left|u(t)-p_{\zeta}^{1} u_{\zeta}(t)\right|=0$

DEMONSTRATION.

$$
\begin{aligned}
& \text { Soit } \Lambda_{\zeta} 1^{\prime} \text { opérateur de } V_{\zeta} \text { dans } V_{\zeta}^{\prime} \times v_{h} \text { défini par : } \\
& \Lambda_{\zeta} u_{\zeta}=\left\{\nabla_{k} u_{\zeta}+A_{\zeta} u_{\zeta}, \quad u_{\zeta}^{1}\right\}=\left\{f_{\zeta}, u_{o h}\right\}
\end{aligned}
$$


Nous allons montrer que les opérateurs $\Lambda_{\zeta}$ sont consistants à l'opérateur $\Lambda$ et que les opérateurs $\Lambda_{\zeta}^{-1}$ sont stables.

Les hypothèses (5-19) impliquant la convergence discrète de $\left\{f_{\zeta}, u_{o h}\right\}$ vers $\left\{\mathrm{f}, \mathrm{u}_{0}\right\}$ dans $W \times \mathrm{H}$, il en résultera, d'après le théorème 2-1 du $\mathrm{n}^{\circ} 2-4$ du $\$ 2$ du chapitre $I$, que les solutions $u_{\zeta}$ convergent discrètement vers la solution $u$ du problème $P$.

Puisque les approximations autoadjointes $A_{h}(V)$ sont stables et convergentes, on en déduit la convercence forte de $p_{\zeta}^{1} u_{\zeta}$ vers $u$ dans l'espace $W$ d'après le théorème $1-1$ du $\$ 1$ du chapitre $I$.

CONSISTANCE DES $\Lambda_{\zeta}$ A $\Lambda$.

I1 faut démontrer que si $\int_{0}^{\mathrm{T}}\left\|\mathrm{p}_{\mathrm{k}} \mathrm{v}_{\zeta}\right\|_{\mathrm{h}}^{2} \mathrm{dt}$ est borné,

$\left|\left(\mathbf{r}_{\zeta}^{*} \Lambda \mathrm{u}-\Lambda_{\zeta} \mathbf{r}_{\zeta}^{*} \mathbf{u}, v_{\zeta}\right)_{\zeta}\right|$ tend vers 0 avec $\zeta$.

Mais cela revient à montrer que $\left|x_{\zeta}\right|+\left|Y_{\zeta}\right|$ tend vers 0 avec $\zeta$, où :

$x_{\zeta}=\int_{0}^{T}\left\{\left(u^{\prime}, r_{\zeta} v_{\zeta}\right)-\left(\nabla_{k} r_{h}^{*} u, p_{k} v_{\zeta}\right)_{h}\right\} d t$

$Y_{\zeta}=\int_{0}^{T}\left\{a\left(t ; u \cdot p_{\zeta} v_{\zeta}\right)-a_{h}\left(t ; p_{k} r_{k} r_{h}^{x} u \cdot p_{k} v_{\zeta}\right)\right\} d t$.

Mais puisque les approximations $A_{h}(V)$ sont autoadjointes,

$\left|x_{\zeta}\right|=1 \int_{0}^{T}\left(u^{\prime}-\nabla_{k} u, p_{\zeta} v_{\zeta}\right) d t\left|\leqslant M \int_{0}^{T}\right|\left|u^{\prime}-\nabla_{k} u\right|_{*}^{2} d t$ tend vers 0.

D'autre part :

$Y_{\zeta}=\int_{0}^{T}\left\{a\left(t ; u, p_{\zeta} v_{\zeta}\right)-a_{h}\left(t ; r_{h}^{*} u, p_{k} v_{\zeta}\right)\right\} d t+$

$+\int_{0}^{T} a_{h}\left(t ; r_{h}^{*}\left(u-p_{k} r_{k} u\right), p_{k} v_{\zeta}\right)$

D'après 1 'hypothèse $(5-18)$, le premier terme tend vers 0 . Puisque les approximations autoadiointes $A_{h}(V)$ sont stables :

$$
\begin{aligned}
& \left|a_{h}\left(t ; r_{h}^{*}\left(u-p_{k} r_{k} u\right), p_{k} v_{\zeta}\right)\right| \leqslant M|| r_{h}^{*}\left(u-p_{k} r_{k} u\right)||_{h}|| p_{k} v_{\zeta} \|\left.\right|_{h} \leqslant \\
& \leqslant M|| u-p_{k} r_{k} u|||| p_{k} v_{\zeta}||_{h} \\
& \text { ce qui entratne que le second terme de } Y_{\zeta} \text { tend vers } o \text {. }
\end{aligned}
$$


Nous avons donc démontré la consistance des $\Lambda_{\zeta}$ à $\Lambda$.

STABILITE DES $\Lambda_{\zeta}^{-1}$.

Supposons que $\left|! f_{\zeta} !\right|_{V_{\zeta}^{\prime}}^{2}+\left|u_{o h}\right|_{h}^{2}$ soit borné. Il résulte du théorème a que sous 1 'hypothèse $(5-15):$

$\sup _{t}\left|p_{k} u_{\zeta}\right|_{h}^{2}+\int_{0}^{T}\left\|n_{k} u_{\zeta}\right\|_{h}^{2} d t \leqslant c s t e$.

Montrons d'autre part que :

$\int_{0}^{T}\left\|\nabla_{k} p_{k} u_{\zeta}\right\|_{* h}^{2} d t \leqslant c s t e$.

En effet, les approximations $A_{k}\left(V_{h}\right)$ étant autoadjointes, on a :

$\left|\left(\nabla_{k} r_{k} u_{\zeta}, v_{h}\right)_{h}\right|=\left|\left(r_{k} \nabla_{k} u_{\zeta}, v_{h}\right)_{h}\right|=\left|\left(\nabla_{k} u_{\zeta}, r_{k}^{*} v_{h}\right)_{\zeta}\right|=$

$=\left|\left(f_{\zeta}, r_{k}^{*} v_{h}\right)-a_{\zeta}\left(u_{\zeta}, r_{k}^{*} v_{h}\right)\right| \leqslant\left(\left\|f_{\zeta}\right\|_{V_{\zeta}}+\int_{0}^{T}\left\|p_{k} u_{\zeta}\right\|_{h}^{2} d t\right)\left\|v_{h}\right\|_{h} \leqslant$

$\leqslant \quad y\left\|v_{h}\right\|_{h}$

Il en résulte donc que :

$\int_{0}^{\mathrm{T}}\left\{\left\|p_{k} u_{\zeta}\right\|_{h}^{2}+\left\|\nabla_{k} p_{k} u_{\zeta}\right\|_{* h}^{2}\right\} d t \leqslant c s t e$.

On en déduit, d'arrès le théorème $2-1$ du chapitre $I$, que les ${ }_{\zeta}$ convergent discrètenent vers $u$ dans $W$, c'est-ì-dire que :

$\int_{0}^{T}\left\{\left\|p_{k} u_{\zeta}-r_{h}^{*} u\right\|_{h}^{2}+\left\|\nabla_{k} p_{k} u_{\zeta}-r_{h}^{*} u^{\prime}\right\|_{h}^{2}\right\} d t$ tend vers 0.

Puisque les approximations $A_{h}(V)$ sont autoadjointes, stables et convergentes, on en déduit que :

$\int_{0}^{T}\left\{\left\|p_{\zeta} u_{\zeta}-u\right\|^{2}+\left\|\nabla_{k} p_{\zeta} u_{\zeta}-u^{\prime}\right\|_{\star}^{2}\right\} d t$ tend vers 0

Ceci établit alors (5-20) puisque $\nabla_{k} p_{\zeta} u_{\zeta}=\frac{d}{d t} p_{\zeta}^{1} u_{\zeta}$.

REMARCUE 5-1.

On aurait pu considérer plus généralement les solutions $u_{\zeta}$ du : 
PROBI,FME $\mathrm{P}_{\zeta}^{\theta}:$

Chercher $u_{\zeta}$ dans $v_{\zeta}$ verifient, si $0 \leqslant \theta \leqslant 1$

$(5-22)\left\{\begin{array}{l}\text { i) }\left(\nabla_{k} u_{\zeta} \cdot v_{\zeta}\right)+a_{\zeta}\left(u_{\zeta}+\theta k \nabla_{k} u_{\zeta} \cdot v_{\zeta}\right)=\left(f_{\zeta} \cdot v_{\zeta}\right) \\ \text { ii) } u_{\zeta}^{1}=u_{o h}\end{array}\right.$

Pour $\theta=0$. on retrouve le schéna explicite, pour $\theta=1 / 2$. celui de Crank-Nicholson, pour $\theta=1$, le schéma imp̣licite.

Les conclusions du théorème 5-l demeurent si $l$ 'on remplace 1 'hỵnothèse (5-15) par :

$(5-23)\left\{\begin{array}{l}\text { i) } M^{2} k S(h)^{2} \leqslant 2 c(1-2 \theta)^{-1}(1-\delta) \text { si } \theta<1 / 2 \\ i i) k . h \text { quclconques si } \theta \geqslant 1 / 2\end{array}\right.$

En effet, d'après P.A. RAVIART ( [1] ) , les conclusions du théorème A demeurent sous 1 'hypothèse $(5-23)$.

REMARQ̣UE 5-2.

Nous avons utilisé la notion d'approximations autoadiointes pour ohtenir la convergence forte de $p_{\zeta}^{1} u_{\zeta}$ vers $u$ dans l'espace $W$.

En prenant des approximations $A_{h}(v)=\left(v \cdot v_{h}, p_{h}, r_{h}\right)$ stables et convergentes. mais non autoadiointes, nous obtenons la conversence faible de $r_{\zeta} u_{\zeta}$ vers $u$ dans $L^{2}(O, T ; V) \cap L^{\infty}(O, T ; H)$. Par des arguments de compacité et d'estimations de dérivées fractionnaires. P.A. RAVIART [1] a montré la convergence forte de $P_{\zeta} u_{\zeta}$ dans l'esnace $L^{2}(0, T ; H)$.

'MAJORATION DE L'ERREUR.

Prenons :

$(5-24)\left\{\begin{array}{l}\left(u_{h}, v_{h}\right)_{h}=\left(p_{h} u_{h}, p_{h} v_{h}\right) \\ a_{h}\left(t ; u_{h}, v_{h}\right)=a\left(t ; p_{h} u_{h}, p_{h} v_{h}\right) \\ \left(f_{h}(t), v_{h}\right)=\left(f(t), p_{h} v_{h}\right) \\ \left(u_{o h}, v_{h}\right)=\left(u_{0}, p_{h} v_{h}\right)\end{array}\right.$ 
La restriction $r_{h}^{*}$ est définie par :

(5-25) $\quad\left(p_{h} r_{h}^{*} f, p_{h} v_{h}\right)=\left(f, p_{h} v_{h}\right)$

et les solutions $u_{\zeta}$ du problème $P_{\zeta}$ sont solutions de

$(5-26)\left\{\begin{array}{l}\left(\frac{d}{d t} p_{\zeta}^{1} u_{\zeta}, p_{\zeta} v_{\zeta}\right)+a\left(t ; p_{\zeta} u_{\zeta}, p_{\zeta} v_{\zeta}\right)=\left(f(t), p_{\zeta} v_{\zeta}\right) \\ \left(p_{\zeta}^{1} u_{\zeta}\right)(0)=r_{h}^{*} u_{0} .\end{array}\right.$

THEORE:AE 5-2.

Sous l'hipnothèse $(5-24)$, les erreurs $u-p_{\zeta}^{1}{ }_{\zeta}$ et $u-F_{\zeta}{ }^{\prime}{ }_{\zeta}$ v'ricient $l^{\prime} i-$ négalité :

$(5-27)\left\{\begin{array}{l}\sup _{t}\left|u(t)-p_{\zeta}^{1} u_{\zeta}(t)\right|^{2}+\int_{0}^{T}\left\|u-p_{\zeta} u_{\zeta}\right\|^{2} d t \leqslant \\ \leqslant M\left[\left(\int_{0}^{T}\left\{\| u-\left.p_{\zeta} r_{\zeta} u\right|^{2}+k|| \nabla_{k} p_{\zeta} u_{\zeta}||^{2}\right\} d t\right)^{1 / 2}+\left|u_{0}-p_{h} r_{h}^{*} u_{0}\right|^{2}\right]\end{array}\right.$

quelle que soit la restriction $r_{h}$ de $v_{h}$ dans $v$.

On remarque en effet que :

$\left(\frac{d}{d t}\left(u-p_{\zeta}^{1} u_{\zeta}\right), u-p_{\zeta}^{1} u_{\zeta}\right)+a\left(t: u-p_{\zeta} u_{\zeta}, u-p_{\zeta} u_{\zeta}\right)=$

$=\left(\frac{d}{d} \bar{t}\left(u-p_{\zeta}^{1} u_{\zeta}\right)+A(t)\left(u-p_{\zeta} u_{\zeta}\right), u-p_{\zeta} r_{\zeta} u\right)+$

$+\left(\frac{d}{d t}\left(u-p_{\zeta}^{1} u_{\zeta}\right), p_{\zeta}^{1} u_{\zeta}-p_{\zeta} u_{\zeta}\right)$

Mais nous avons vu $\left(\$ 3, n^{\circ} 3-3,(3-43)\right)$ que si $u_{k}^{\mu}=p_{h} u_{\zeta}^{\mu}$,

$$
\begin{aligned}
& \int_{0}^{T}\left\|p_{k}^{1} u_{k}-p_{k} u_{k}\right\|^{2}=k \int_{0}^{T}\left\|\sum_{\mu}\left(\nabla_{k} u_{k}\right)^{\mu}\left(\frac{t}{h}-\mu+1\right) k^{1 / 2} x_{k}^{\mu}\right\|^{2} \leqslant \\
& \leqslant k \int_{0}^{T}\left\|\nabla_{k} p_{k} u_{k}\right\|^{2} d t .
\end{aligned}
$$

On en déduit alors (5-27) en intégrant cette inégalité.

5-2. APPROXIMATION DES SOLUTIONS DES EQUATIONS PARABOLIQUES.

Prenons pour espace $V$ l'espace $H_{0}^{l}(\Omega)$, pour espace $H l^{\prime}$ espace $L^{2}(\Omega)$ et pour forme a $(t ; u, v)$ la forme 
$(5-28)\left\{\begin{array}{l}a(t ; u, v)=\sum_{1, j=1}^{n} \int_{\Omega} a_{1 j}(x, t) D_{1} u \cdot \overline{D_{j} v} d x+ \\ +\sum_{i=1}^{n} \int_{\Omega} a_{i}(x, t) D_{i} u \cdot \bar{v} d x+\int_{\Omega} a_{0}(x, t) u \bar{v} d x\end{array}\right.$

où les coefficients $a_{i j}(x, t), a_{i}(x, t), a_{0}(x, t)$ appartiennent à $L^{\infty}(\Omega \times(0, T))$ et vérifient :

(5-29) $\operatorname{Re}\left[a_{i j}(x, t) \zeta_{i} \bar{\zeta}_{j} \geqslant c|\zeta|^{2} ;\right.$ pour presque tout $x, t$.

Il existe alors une solution unique $u(x, t)$ du problème de Cauchy-Dirichlet :

PROBLE:TE P .

$(5-30)\left\{\begin{array}{l}i) \frac{\partial u}{\partial t}-\sum_{i, j=1}^{n} D_{f}\left(a_{1 j}(x, t) D_{1} u\right)+\sum_{i=1}^{n} a_{i}(x, t) D_{1} u+a_{0}(x, t) u=f(t) \\ \text { ii) } u(x, 0)=u_{0}(x) \text { dans } L^{2}(\Omega) \\ \text { iii) }\left.u(x, t)\right|_{\Gamma}=0 \quad \text { : } \Gamma \text { est la frontiere de } \Omega .\end{array}\right.$

Soit $\vec{L}=\left(L_{0}, L_{i}, \ldots, L_{n}\right) n+1$ multientiers tels que $L_{i} \geqslant e_{i}$. Solt

$L=\sup _{0 \leqslant i \leqslant n} L_{i} \cdot$

Prenons pour espace $v_{h}$ 1'espace $w_{o, h}^{1,2}(\Omega, L)$ et pour produit scalaire $\left(u_{h}, v_{h}\right)_{h}$.

$(5-31) \quad\left(u_{h}, v_{h}\right)_{h}=\sum_{\alpha} u_{h}^{\alpha}-\bar{v}_{h}^{\alpha}$

Considérons les prolongements $p_{h}^{L_{i}} u_{h}, p_{h}^{L} u_{h}$ définis au $n^{\circ} 3-2$ du $\$ 3$, et pour restriction $r_{h}$ la restriction $r_{h}^{* L}$. Les approximations

(5-33) $\quad A_{h}(v)=\left(v, v_{h}, p_{h}^{L}, r_{h}^{* L}\right)$

sont autoadjointes. stables et convergentes si 1 'ouvert $\Omega$ est suffisamment régulier, d'après le théorème $3-2$ du $n^{\circ} 3-2$ du $\$ 3$.

Prenons pour forme $a_{h}\left(t ; u_{h}, v_{h}\right)$ la forme suivante: 
$(5-34)\left\{\begin{array}{l}a_{h}\left(t: u_{h}, v_{h}\right)=\sum_{1, j=1}^{n} \int_{\Omega} a_{i j}(x, t) D_{i} p_{h}^{L_{1}} u_{h}, D_{j} p_{h}^{L_{j}} v_{h} d t+ \\ +\sum_{i=1}^{n} \int_{\Omega} a_{i}(x, t) D_{1} p_{h}^{L_{1}} u_{h} p_{h}^{L_{0}} v_{h} d t+\int_{\Omega} a_{0}(x, t) p_{h}^{L_{0}} u_{h} p_{h}^{L_{0}} v_{h} d t\end{array}\right.$ Prenons

$$
\left(f_{h}(t), v_{h}\right)_{h}=\left(f(t), p_{h}^{L_{0}} v_{h}\right) \text { et }\left(u_{o h}, v_{h}\right)_{h}=\left(u_{0}, p_{h}^{L_{0}} v_{h}\right) \text {. }
$$

D'après le théorème $2-1$ du $\$ 2$ de ce chapitre, la fonction $S(h)$ est majorée par $N\left(\sum_{i=1}^{n} h_{i}^{-2}\right)^{\frac{1}{2}}$

On déduit du théorème 5-1 et des résultats du n 3-2 1a :

PROPOSITION 5-1.

Supposons que (5-31), (5-34) et (5-35) aient lieu. Si

$(5-36) \quad k \sum_{i=1}^{n} h_{i}^{-2} \leqslant 2 \mathrm{~cm}^{-2} \mathrm{~N}^{-2}(1-\delta), \delta>0$

les soiutions $u_{\zeta}$ de l'équation variationnelle :

$(5-37)\left\{\begin{array}{l}\int_{0}^{T}\left\{\left(\nabla_{k} p_{k} u_{\zeta} \cdot p_{k} v_{\zeta}\right)_{h}+a_{h}\left(t ; p_{k} u_{\zeta}, p_{k} v_{\zeta}\right)\right\} d t=\int_{0}^{T}\left(f_{h}(t), p_{k} v_{\zeta}\right) d t \\ u_{\zeta}^{1}=u_{o h}\end{array}\right.$ convergent vers la solution $\mathrm{u}$ de $(5-30)$ au sens suivant :

$(5-38)\left\{\begin{array}{l}P_{k} P_{h}^{L} u_{\zeta} \text { converge vers } u \text { dans } L^{2}(0, T: V) \text { fort. } \\ \nabla_{k} p_{k} p_{h}^{L} u_{\zeta}=\frac{d}{d t} p_{k}^{1} P_{h}^{L} u_{\zeta} \text { converae vers } u^{\prime} \text { dans } L^{2}\left(0, T ; V^{\prime}\right) \text { fort. } \\ P_{k}^{1} P_{h}^{L} u_{\zeta} \text { converge vers } u \text { dans } C(0, T ; H) \text { fort. }\end{array}\right.$ Les matrices associées aux opérateurs $A_{h}$ ont été explicitées au $n^{\circ} 4-2$ du $\$ 4$.

5-3. CONSTRUCTION DE SCHEMAS A PLUSIEURS NIVEAUX - MAJORATION DE L'ERREUR.

La solution $u$ de l'équation $u^{\prime}+A(t) u=f, u(0)=u_{0}$ est solution du : 
PROBLEME P .

Chercher $\mathrm{u}$ dans $\mathrm{L}^{2}(0, \mathrm{~T}, \mathrm{~V})$ verrifiant:

$\left\{\begin{aligned} E(u-39) & =\int_{0}^{T}\left\{-\left(u, \phi^{\prime}\right)+a(t: u, 0)\right\} d t=F(\phi)= \\ & =\int_{0}^{T}\left(f(t) \cdot \phi(t) d t+\left(u_{0}, \phi(0)\right)\right.\end{aligned}\right.$ pour toute fonction $\phi$ de l'espace $\Phi$ :

$(5-40) \Phi=\left\{\phi \varepsilon L^{2}(0, T ; V) ; \phi^{\prime} \varepsilon L^{2}\left(0, T: V^{\prime}\right): \phi(T)=0\right\}$.

(cf. $\mathrm{n}^{\circ}$ 5-1 du $\$ 5$ du chapitre I ) .

L'existence d'une solution du problème $P$ peut être établie à l'aide du lemme des projections (cf. J.L. LIONS [1] et le $n^{0} 3-6$ du $\$ 3$ du chapitre $I$. théorème 3-6). Nous allons utiliser ce théorème pour construire des problèmes approchés et montrer la convernence forte de leurs solutions.

Nous apnrocherons le problème $P$ posé dans $L^{2}(0, T ; V)$ par des problèmes $P_{k}$ posés dans $V$ (semi-discrétisation par rapport à la variable de temps). La discrétisation complète s'obtient alors aisément en introduisant des approximations $A_{h}(V)$ et en utilisant les résultats du $\$ 3$ et du $\$ 5$ du chapitre I . Nous n'expliciterons pas ici cette deuxième étape de l'approximation, aui n'offre aucune difficulté.

Posons :

$(5-41) \quad\|\phi\|_{V}=\left(\int_{0}^{T}\|\phi(t)\|^{2} d t\right)^{1 / 2} ;\|\| \phi \|=\left(\|\phi\|_{V}+|\phi(0)|^{2}\right)^{1 / 2}$

Soit $L$ un entier $\geqslant 1$. Posons :

$(5-42)\left\{\begin{array}{l}R_{k}^{L}(0, T)=\left\{\alpha \varepsilon N ; 1 \leqslant \alpha \leqslant T k^{-1}+\mathrm{L}+1\right\} \\ V_{k}=\left\{u_{k}=\left(u_{k}^{\alpha}\right) ; u_{k}^{\alpha} \varepsilon V: 1 \leqslant \alpha \leqslant T k^{-1}+L+1\right\} \\ \phi_{k}=\left\{\phi_{k}=\left(\phi_{k}^{\alpha}\right) ; \phi_{k}^{\alpha} \varepsilon V: \phi_{k}^{\alpha}=0 \text { pour } \alpha \geqslant T_{k}^{-1}\right\}\end{array}\right.$

On introduit les prolongements $p_{k}=p_{k}^{L}$ :

(5-43) $\quad p_{k}^{L} u_{k}=\sqrt{k} \sum_{\alpha=1}^{T k^{-1}+L+1} \quad u_{k}^{\alpha} x_{L+1, k}^{\alpha} \quad(t)$. 
Si $\pi$ est le prolongement de Babitch (par exemple; cf. $\$ 3, n^{\circ} 3-3$, exemple 3-1), et $s i \gamma_{k}$ est une fonction nulle pour $t \geqslant T-(L+1) k$, tendant vers 1 dans $\mathrm{w}^{1, \infty}(-\infty, \mathrm{T})$, on introduit les restrictions :

(5-44) $\quad r_{k} \phi=\sqrt{k}\left(\pi \phi, x_{k}^{\alpha}\right)_{\alpha} ; s_{k} \phi=\sqrt{k}\left(r_{k} \pi \phi, x_{k}^{\alpha}\right)_{\alpha}$

On considère enfin les normes :

(5-45) $\quad\left\|\phi_{k}\right\|_{k}=\left\|p_{k}^{L} \phi\right\|_{v} ; \quad\|\| \phi_{k}\left\|_{k}=\right\| p_{k}^{L} \phi_{k}\|\|$

Nous nous posons alors le:

PROBLEME $\mathrm{P}_{\mathrm{k}}$.

Chercher $u_{k}$ dans $v_{k}$ vérifiant:

$(5-46)\left\{\begin{array}{l}E\left(u_{k}, \phi_{k}\right)=\int_{0}^{T}\left\{a\left(t ; p_{k}^{L} u_{k}, p_{k}^{L} \phi_{k}\right)-\left(p_{k}^{L} u_{k}, p_{k}^{L} \phi_{k}\right)\right\} d t= \\ =F_{k}\left(\phi_{k}\right)=\int_{0}^{T}\left(f(t), p_{k}^{L} \phi_{k}\right) d t+\left(u_{0}, p_{k}^{L} \overline{\phi_{k}}(0)\right) .\end{array}\right.$

pour toute fonction $\phi_{\mathrm{k}}$ de $\Phi_{\mathrm{k}}$.

Avant de montrer que des solutions $u_{k}$ du problème $P_{k}$ conversent vers 1a solution $u$ du problème $P$, nous allons expliciter le schéma auquel conduit l'équation (5-46)dans le cas où $A(t)=A$ ne dépend pas de $t$ pour simplifier un peu les calculs.

Rappelons que :

$x_{L+1}(t)=\sum_{k=0}^{L} \psi_{L}^{k}(t+L-k) x^{-L+k}(t)$

Posons, si $\alpha$ et ? sont des entiers :

$(5-47)\left\{\begin{array}{l}\Gamma_{\alpha}^{q}=\sum_{r=\sup (L+1-\alpha, q)}^{L} \int_{-1}^{0} \psi_{L}^{r-q}(t) \cdot \frac{d}{d t} \psi_{L}^{r}(t) d t \cdot s i \quad q \geqslant 0 . \\ r_{\alpha}^{q}=\sum_{r=\sup (L+1-\alpha, 0)}^{L+q} \int_{-1}^{0} \psi_{L}^{r-q}(t) \cdot \frac{d}{d t} \psi_{L}^{r}(t) d t \cdot \text { si } q<0 . \\ \Omega_{\alpha}^{q}=\sum_{r=\sup (L+1-\alpha, q)}^{L} \int_{-1}^{0} \psi_{L}^{r-q}(t) \cdot \psi_{L}^{r}(t) d t \cdot s i \quad q \geqslant 0 . \\ \Omega_{\alpha}^{q}=\sum_{r=\operatorname{Lup}(L+1-\alpha, 0)}^{L} \int_{-1}^{0} \psi_{L}^{r-q}(t) \cdot \psi_{L}^{r}(t) d t \cdot \text { si } q<0 .\end{array}\right.$

Les expressions $\Gamma_{\alpha}^{q}$ et $\Omega_{\alpha}^{q}$ ne dépendent pas de $\alpha$ si $\alpha \geqslant L+1$ et sont nulles si $|q| \geqslant L$. Si $\alpha<L+1$, ces expressions dépendent de $\alpha$ et sont 
nulles si $q \leqslant 1-\alpha$ et $q \geqslant L$.

PROPOSITION 5-2.

Les solutions $u_{k}=\left(u_{k}^{\alpha}\right)_{1 \leqslant \alpha \leqslant T k}{ }^{-1}+L+1$ du problème $P_{k}$ vérifient:

$(5-48)\left\{\begin{array}{c}\text { i) } u_{k}^{\alpha} \quad \text { arbitraires si } 1 \leqslant \alpha \leqslant L . \\ \text { ii) } \sum_{1-\alpha}^{L}\left(k^{-1} \Gamma_{\alpha}^{q}+A \Omega_{\alpha}^{q}\right) u_{k}^{\alpha+q}=k^{-1} a_{L}(L+1-\alpha, 0)+f_{k}^{\alpha} \text { si } 1 \leqslant \alpha \leqslant L \\ \text { iii) } \sum_{-L}^{+L}\left(k^{-1} \Gamma^{q}+A \Omega^{q}\right) u_{k}^{\alpha+q}=f_{k}^{\alpha} \text { si } L+1 \leqslant \alpha \leqslant T k^{-1}\end{array}\right.$

I1 en résulte donc que les $u_{k}$ sont solutions d'un schéma à $2 L+1$ "niveaux", implicite; Seule la donnée des $L$ premières composantes $u_{k}^{1}, \ldots, u_{k}^{L}$ est arbitraire, les $\mathrm{L}+1$ autres composantes du "démarrage" étant déterminées par (5-48) ii).

Il existe donc des solutions du problème $P_{k}$, uniques si les $L$ premières composantes de la suite $u_{k}$ sont fixées.

THEOREME 5-3.

Il existe des composantes $u_{k}^{1}, \ldots, u_{k}^{L}$ telles que les solutions $u_{k} d u$ proble me $\mathrm{P}_{\mathrm{k}}$ convergent fortement dans $\mathrm{L}^{2}(0, \mathrm{~T} ; \mathrm{V})$ vers la solution $\mathrm{u}$ du problème $\mathrm{P}$ :

(5-49) $\lim _{k=0} \int_{0}^{T}\left\|u(t)-p_{k}^{L} u_{k}(t)\right\|^{2} d t=0$.

L'erreur $u-p_{k}^{L} u_{k}$ est majorēe par:

(5-50) $\left\|u-p_{k}^{L} u_{k}\right\|_{V} \leqslant M\left(\left\|u-p_{k}^{L} r_{k} u\right\|_{V}^{2}+\left\|\frac{d}{d t}\left(u-p_{k}^{L} r_{k} u\right)\right\|_{V}^{2}\right)^{1 / 2}$.

Vérifions que les hypothèses (3-50) et (3-53) du théorème 3-6 du n 3-6 du $\$ 3$. chapitre I sont satisfaites.

Par intégration par parties, puisque $\mathrm{p}_{k}^{L} \phi_{k}(T)=0$, on obtient :

$(5-51)\left\{\begin{aligned} 2 \operatorname{Re} E_{k}\left(\phi_{k}, \phi_{k}\right) & \geqslant \inf (1, c) \int_{0}^{T}\left[\left\|p_{k}^{L} \phi_{k}\right\|^{2} d t+\left|p_{k}^{L} \phi_{k}(0)\right|^{2}\right] \\ & =\inf (1, c)\left\|\phi_{k}\right\| \|_{k}^{2}\end{aligned}\right.$ 
D'autre part :

(5-52) $\left|F_{k}\left({ }_{k}\right)\right| \leqslant 1 / 2|| f||_{V},|| p_{k}^{L} \phi_{k}\left\|_{V}+\left|u_{o}\right|\left|p_{k}^{L} \phi_{k}(0)\right| \leqslant c^{\prime}\right\|\left\|\phi_{k}\right\| \|_{k}$.

Iontrons maintenant aue :

(5-53) $\sup _{d_{k}}\left\|\left.\right|_{k} !\right\|_{k}^{-1} \mathrm{E}\left(\mathrm{u}-p_{k}^{L} \mathbf{r}_{k} u, p_{k}^{L} \phi_{k}\right)$ tend vers 0 avec $k$.

?ais, en intérant par parties, on obtient :

$(5-54) E(u, \infty)=\int_{0}^{T}\left\{\left(u^{\prime}, \$\right)+a(t \cdot u, D)\right\} d t+(u(0), \phi(0))$ si bien gue si $\tau_{k} u=u-p_{k}^{L} r_{k} u$, on a :

$(5-55)\left\{\begin{array}{l}\left|E\left(\tau_{k} u \cdot p_{k}^{L} t_{k}\right)\right| \leqslant \\ \leqslant 1\left(|| \tau_{k} u\left\|_{V}^{2}+|| \frac{d}{d t}\left(\tau_{k} u\right)\right\|_{V^{\prime}}^{2}+\left|\tau_{k} u(0)\right|^{2}\right)^{1 / 2}\|\| t_{k}\|\|_{k}\end{array}\right.$

ce rui établit (5-53) . Le théorème 3-6 du $n^{\circ} 3-6$ du $\$ 3$, chapitre I entrafne 1a convergence forte de $n_{k}^{L} u_{k}$ vers u dans $V$.

n'autre part. si $\varepsilon_{k}=u_{k}-r_{k} u$, on a :

(5-56) $\quad E_{k}\left(\varepsilon_{k} \cdot \phi_{k}\right)=E\left(p_{k}^{L} \varepsilon_{k} \cdot p_{k}^{L} \phi_{k}\right)=E\left(\tau_{k} u, p_{k}^{L} \phi_{k}\right)=G_{k}\left(\phi_{k}\right)$

D'après (5-55), 1a norme $\left\|\left|G_{k}\right|\right\|$ de $c_{k}$ est majorée par

(5-57) \|\|$c_{k} \| \leqslant M\left(\left\|\tau_{k} u\right\|_{l y}^{2}+\left\|\frac{d}{d t} \tau_{k} u\right\|_{V^{\prime}}^{2}\right)^{1 / 2}$

Duisque $|u(0)|^{2} \leqslant M\left(\left|! u\left\|_{v}^{2}+|| u^{\prime}\right\|_{V^{\prime}}^{2}\right)^{\frac{1}{2}}\right.$.

Il en résulte alors que :

(5-58) $\left\|n_{k}^{L} u_{k}-n_{k}^{L} r_{k} u\right\| !_{V} \leqslant M\left\|\left.\right|_{k}\right\| \leqslant M\left(\left\|\tau_{k} u\right\|^{2}+\left\|\left(\tau_{k} u\right) \cdot\right\|_{V^{\prime}}^{2}\right)^{1 / 2}$ ce rui achève la démonstration du théorème.

EXE'TPLE 5-1.

in prenant $\mathrm{L}=1$. nous obtenons le schéma suivant :

(5-59) $\frac{u_{k}^{\alpha+1}-u_{k}^{\alpha-1}}{2 k}+A\left(\frac{u_{k}^{\alpha+1}}{6}+\frac{u_{k}^{\alpha}}{3}+\frac{u_{k}^{\alpha-1}}{6}\right)=f_{k}^{\alpha} \quad$ si $\quad \alpha \geqslant 1$.

On retrouve un schéma décrit dans v. SAULEV [1] . 
RAPPELS DE THEOREMES D'ISO:HORPHIS:TE ET D' INTERPOLATION.

1) LE TRIPLET $A=\{\mathrm{v}, \mathrm{H}, \mathrm{a}(\mathrm{u}, \mathrm{v})\}$.

Nous désignerons par $A=\{\mathrm{V}, \mathrm{H}, \mathrm{a}(\mathrm{u}, \mathrm{V})\}$ la donnée de deux espaces de Hilbert $V$ et $H$ et d'une forme $a(u, v)$ vérifiant :

a) $\mathrm{V}$ est contenu dans $\mathrm{H}$ avec une topoloaie plus fine. $\mathrm{V}$ est ciense dans $\mathrm{H}$. Les produits scalaires et les normes de $\mathrm{H}$ et de $\mathrm{V}$ sont respectivement notés :

(1) $(f, g):|f|=(f, f)^{1 / 2} ;((u \cdot v)) ;|| u||=((u, u))^{1 / 2}$

b) La forme sesquilinéaire a $(\mathrm{u} . \mathrm{v})$ est continue sur $\mathrm{v} \times \mathrm{V}$ :

(2) $|\mathrm{a}(\mathrm{u}, \mathrm{v})| \leqslant M\|\mathbf{u}\|\|\mathbf{v}\|$.

2) LES ESPACES V ET H .

On Identifiera toujours l'espace $H$ à son anti-dual par le produit scalaire ( $f$. g). Puisque $\mathrm{V}$ est dense dans $\mathrm{H}$, l'espace $\mathrm{H}$ s'identifie $\bar{a}$ un sousespace dense de $l^{\prime}$ anti-dual $V^{\prime}$ de $V$.

(pour la dualité définie par le produit scalaire $(f, \xi)$ ).

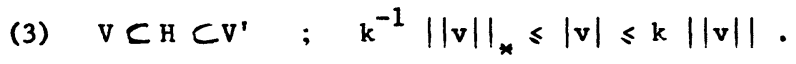

Si $\Lambda$ est l'isomorphisme canonique de $V$ sur $V^{\prime}, J$ son inverse, $V^{\prime}$ est muni d'une structure hilbertienne définie par :

$$
((\mathbf{u}, \mathbf{v}))_{*}=((\mathrm{J} \mathbf{u}, \mathrm{J} \mathbf{v}))=(\mathrm{J} \mathbf{u}, \mathbf{v})
$$

3) LES OPERATEURS DEFINIS PAR $A=\{\mathrm{v}, \mathrm{H}, \mathrm{a}(\mathrm{u}, \mathrm{v})\}$.

Tout d'abord, la forme a $(u, v)$ étant continue sur $v$, elle définit un opérateur continu $A$ de $V$ dans $V^{\prime}$ par :

(4) $(\mathrm{A} \mathrm{u}, \mathrm{v})=\mathrm{a}(\mathrm{u}, \mathrm{v})$ pour tout $\mathrm{v}$ de $\mathrm{V}$.

Le triplet A définit aussi un opérateur non borné sur $H$. On désigne par

$D$ ( A ) le sous espace des $u \in V$ tels que les formes $A$ :

(5) $\quad$ A u : v $\longrightarrow a(u, v) ; u \in v$ 
sont continues pour la topologie induite par H . L'espace $V$ étant dense dans $\mathrm{H}$, elles se prolongent à $\mathrm{H}$ tout entier. •

En munissant D (A) de la norme du graphe :

(6) $|u|_{D(A)}=\left(|u|^{2}+|A u|^{2}\right) ; u \in D(A)$

A est un opérateur continu de $\mathrm{D}$ ( A ) dans $\mathrm{H}$.

On désignera par $A^{*}$ le triplet défini par

(7) $\mathbf{A}^{*}=\left\{\mathbf{v}, \mathbf{H}, \mathbf{a}^{*}(\mathrm{u}, \mathrm{v})\right\} \quad$; $\mathbf{a}^{*}(\mathbf{u}, \mathbf{v})=\mathbf{a} \overline{(\mathrm{v}, \mathrm{u})}$

4) THEOREME D'ISOMORPHISME.

Supposons que $\mathbf{A}=\{\mathbf{V}, \mathbf{B}, \mathbf{a}(\mathbf{u}, \mathbf{v})\}$ soit coercif:

(8) $\operatorname{Re} a(v, v) \geqslant c\|v\|^{2} ; c>0 ; v \varepsilon v$.

Alors :

(9). $\mathrm{D}(\mathrm{A})$ et $\mathrm{D}\left(\mathrm{A}^{*}\right)$ sont denses dans $\mathrm{V}$ et $\mathrm{H}$ et sont des espaces de Hilbert pour la norme du graphe.

(10) - $A^{*}$ est $l^{\prime}$ adjoint de A.

(11) - A est un isomorphisme de V sur $\mathbf{V}^{\prime}$, de $\mathrm{D}$ ( A) sur $\mathbf{H}$ et de $\mathrm{H}$ sur $D\left(A^{*}\right)^{\prime}$.

Commentons ce dernier point. Puisque $\mathrm{D}\left(\mathrm{A}^{*}\right)$ est dense dans $\mathrm{V}$ et $\mathrm{H}$, nous pouvons faire les identifications suivantes :

$D\left(A^{*}\right) \subset V \subset H \subset V^{\prime} \subset D\left(A^{*}\right)^{\prime}$

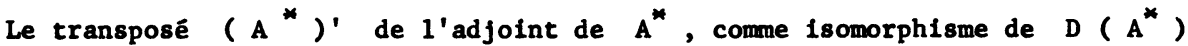
sur $H$, est un isomorphisme de $H$ sur $D\left(A^{*}\right)^{\prime}$ et $\left(A^{*}\right)^{\prime}$ prolonge $A$ de $D(A)$ à $H$, puisque $\left(A^{*}\right)^{\prime}=A$ sur $D(A)$. Ceci justifle le fait de poser $\left(A^{*}\right)^{\prime}=A$.

5) ESPACES D'INTERPOLATIONS (cf. J.L. LIONS - J. PEETRE [1] ) .

Solent $A_{0}$ et $A_{1}$ deux espaces de Banach, $A_{0} \subset A_{1}, A_{0}$ dense dans $A_{1}$; On définira l'espace :

(13) $S\left(\theta, A_{0}, A_{1}\right) ; 0<\theta<1$ 
comme $I^{\prime}$ espace des intégrales des fonctions $\phi(x)$ vérifiant :

$$
\exp (\theta x) \phi(x) \varepsilon L^{2}\left(A_{0}\right) ; \exp ((\theta-1) x) \phi(x) \in L^{2}\left(A_{1}\right)
$$

muni de la norme :

(15) $\|u\|_{S\left(\theta, A_{0}, A_{1}\right)}=\inf _{\phi}\left\{\max \left(\left\|e^{\theta x} \phi(x)\right\|_{L^{2}\left(A_{0}\right)},\left\|e^{(\theta-1) x} \phi(x)\right\|_{L}{ }^{2}\left(A_{1}\right)\right) ;\right.$ $\left.u=\int \phi(x) d x\right\}$

On posera $S\left(0, A_{0}, A_{1}\right)=A_{0}, S_{1}\left(1, A_{0}, A_{1}\right)=A_{1}$.

Les espaces $S\left(\theta, A_{0}, A_{1}\right)$ sont des exemples d'espace $d$ 'interpolation entre $A_{0}$ et $A_{1}$, c'est-à-dire d'espaces vérifiant le :

Théorème d'interpolation :

Si $A \in L\left(A_{0}, B_{0}\right) \cap L\left(A_{1}, B_{1}\right)$, alors $A \in L\left(S\left(\theta, A_{0}, A_{1}\right)\right.$, $\left.s\left(\theta, B_{0}, B_{1}\right)\right)$ et :

(16) ||$A\left\|_{L\left(S\left(\theta, A_{0}, A_{1}\right), S\left(\theta, B_{0}, B_{1}\right)\right)} \leqslant\right\| A\left\|_{L\left(A_{0}, B_{0}\right)}^{1-\theta} \quad\right\| A \|_{L\left(A_{1}, B_{1}\right)}^{\theta}$

Ces espaces vérifient en outre :

(17) $\quad S\left(\theta, A_{0}, A_{1}\right)^{\prime}=S\left(1-\theta, A_{1}^{\prime}, A_{0}^{\prime}\right)$

(18) $\quad H=S\left(\frac{1}{2}, V, V^{\prime}\right)$ 
J.P. AUBIN

[1] Etude d'une certaine classe d'équations aux dérivées partielles non 1inéaires. CR. Acad. Sciences. 256 (1963) p. 350 - 352 .

[2] Un théorème de compacité. CR. Acad. Sciences. 256 (1963) p. 5042 - 5044.

[3] Evaluation des erreurs de troncature dans les espaces de Sobolev. A paraftre dans S.I.A.M.

[4] Behavior of the error of the approximate solutions of boundary value problems for linear elliptic operators by Galerkin's and finite difference methods - A paraftre dans Annali della scola Norm. Pisa.

[5] Non linear Stability - Approximation of solutions of non linear operational equations - A paraître.

[6] Approximation of solution of variational inequations - In Optimization and functional analysis - Academic Press (1967).

[7] Interpolation et Approximation optimales. A paraftre.

N. BOURISAKI

[1] Espaces vectoriels topologiques - Hermann.

J. CEA

[1] Approximation variationnelle des problèmes aux limites. Annales Inst. Fourier 14 (1964) p. $345-444$.

[2] A paraitre.

[3j Séminaire J. CEA. J.L. LIONS. (1965) •

Y.K. DLMIANOVITCH

[1] D.A.N. 159 (1964) p. $250-253$.

J. DIEUDONNE

[1] On biorthogonal Systems - :iichig. Math. J. 2 (1954) p. $7-20$.

N. DUNFORD - J. SCHWARZ

[1] Linear Operators (I). Interscience Publishers (1958). 
E. GAGLIARDO

[1] Proprieta di alcune classi di funzioni in piu variabili. Ren. Sem. Mat di Padova - 27 (1957) p. $284-305$.

S.K. GODUNOV - V.S. RYABENKII

[1] Introduction à la théorie des Schémas aux différences finies - Moscou (1962) .

P. HARTMANN - G. STAMPACCHIA

[1] A paraftre.

0.A. LADYJENSKAYA

11 Problèmes mixtes pour les équations hyperboliques - Moscou.

P.D. LAX

11) Cf. R.D. RICHTMYER - Difference methods for initial value Problems - Interscience Publishers. (1957).

J. LERAY - J.L. LIONS

[1] Quelques résultats de Visik sur les problèmes elliptiques non linéaires par les méthodes de Minty-Browder - Bull Soc. Mat. Fr. 93 (1965) p. 97 107 .

J.L. LIONS

[1] Equations différentielles opérationnelles. Springer Verlag (1961) •

[2] Problèmes aux limites dans les équations aux dérivées partielles. Editions de l'université de Montréal (1962).

[3] Sur les problèmes mixtes pour certains systèmes parabolíques dans les ouverts non cylindriques. Ann. Inst. Fourier. 7 (1957) p. $143-182$.

[4] Dérivées intermédialres et espaces intermédiaires. CR. Acad. Sciences 256 (1963) p. 4343 - 4345 .

J.L. LIONS - J. PEETRE

[1] Sur une classe d'espaces d'Interpolation. Pub. Math. Inst. Htes. Et. Scienti. $\mathrm{n}^{\circ} 19$.

L. NIRENBERG

[1] On elliptic partial differential equations. Cours de Pise (1958). P.A. RAVIART

[1] Sur 1'approximation de certaines équations d'évolution linéaires et non linéaires. (Thèse à paraftre). 
V.K. SAULIEV

[1] Intégration des équations du type parabolique par la méthode des réseaux Moscou (1960).

I.J. SCHOENBERG

[1] On interpolation by spline functions and its minimal properties. Inter series of Num. Math. 5 - 109 - 129 (1964).

L. SCHWARTZ

[1] Théorie des distributions (Hermann).

S.L. SOBOLEV

[1] Congrès I.F.I.P. (1965) .

12] Certaines applications de l'analyse fonctionnelle à la physique mathématique. (Léningrad 1950 ).

[3] Estimation de certaines sommes pour les fonctions définies sur un réseau. Isv. Acad. Nauk. (Ser Mat) 1 (1957) p. $81-86$.

G. STAMPACCHIA

[1] Equations elliptiques du second ordre à coefficients discontinus. Collège de France (1964).

[2] Formes bilinéaires coercitives sur les ensembles convexes CR. Acad. Sciences 258 (1964) p. 4413 - 4416 .

V. THOMEE

[1] Elliptic difference operators and Drichlet's problem. Contributions to differentiel equations. III (1964) p. $301-324$.

M.M. VAINBERG

[1] Variational Methods for the study of non linear operators - Holden Day Inc. (1964). 